\title{
Molecular Mechanisms of Germ Plasm Anchoring in the Early Zebrafish Embryo
}

\author{
Doctoral Thesis
}

for the award of the degree

"Doctor rerum naturalium" (Dr. rer. nat.)

of the Georg-August-Universität Göttingen

within the doctoral program "Genes and Development"

submitted by

\author{
Alexander Goloborodko \\ born in Poltava, Ukraine
}

Göttingen, September 2019 


\section{Thesis Committee}

Dr. Roland Dosch (supervisor, $1^{\text {st }}$ reviewer), Department of Human Genetics, Georg August University Göttingen

Prof. Dr. Fred Wouters ( $2^{\text {nd }}$ reviewer), Laboratory for Molecular and Cellular Systems, Institute for Neuropathology, University Medical Center Göttingen

Prof. Dr. Herbert Jäckle, Emeritus Group of Molecular Developmental Biology, Max Planck Institute for Biophysical Chemistry, Göttingen

\section{Members of the Examination Board}

Prof. Dr. Gregor Bucher, Department of Developmental Biology, Georg-August-Universität Göttingen, Göttingen Center for Molecular Biosciences

Dr. Ufuk Günesdogan, Department of Developmental Biology, Georg-August-Universität Göttingen, Göttingen Center for Molecular Biosciences

Dr. Gerd Vorbrüggen, Laboratory of Molecular Cell Dynamics Cell Migration and Adhesion, Max Plack Institute for Biophysical Chemistry

Date of the thesis defense: 30.10 .2019 


\section{Affidavit}

Herewith, I declare that I prepared the $\mathrm{PhD}$ thesis "Molecular Mechanisms of Germ Plasm Anchoring in the Early Zebrafish Embryo" on my own and with no other sources and aids than quoted.

Göttingen 15.09.2019

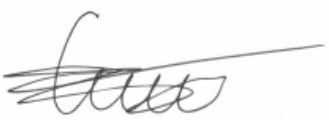

Alexander Goloborodko 


\section{Table of Contents}

Acknowledgments

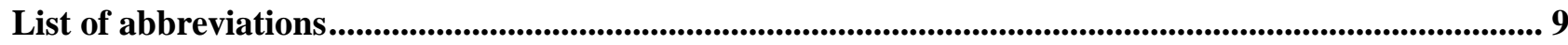

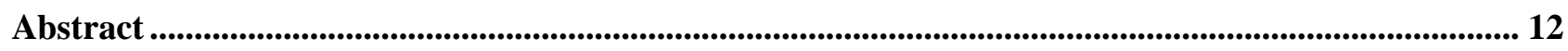

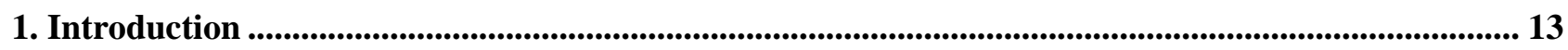

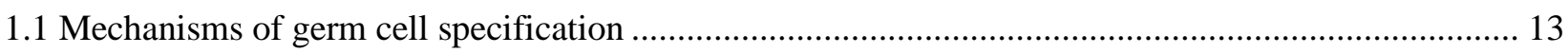

1.2 Germ plasm and its role in germ cell specification ................................................................... 14

1.2.1 Germ plasm localizes to the Balbiani body during early oogenesis .......................................... 14

1.2.2 The role of phase-separation in the Balbiani body and germ plasm assembly .............................. 15

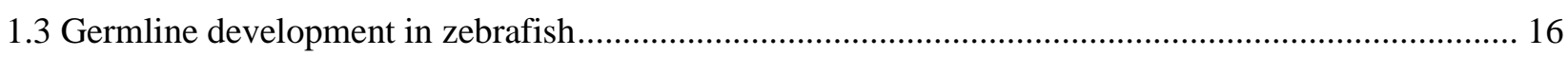

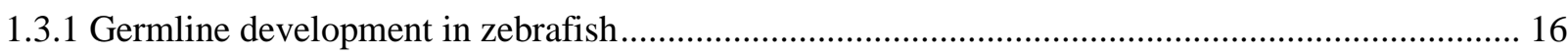

1.4 Molecular mechanisms of germ plasm localization during zebrafish embryogenesis ........................ 18

1.4.1 Role of cytoskeleton in early germ plasm aggregation.......................................................... 18

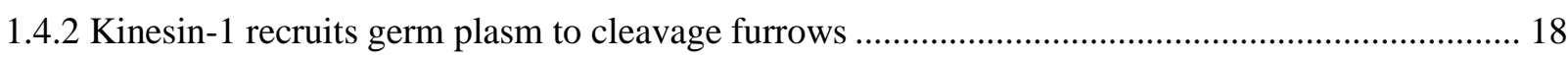

1.4.3 Germ plasm compaction requires furrow microtubule array .................................................. 19

1.4.4 Asymmetric distribution of germ plasm is mediated by the spindle .......................................... 21

1.4.5 Localization of protein components of germ plasm ................................................................. 21

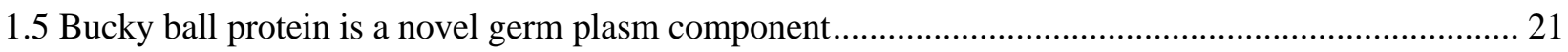

1.5.1 Buc protein is required for germ plasm assembly and localization ............................................. 22

1.5.2. $77 \mathrm{~N}$-terminal amino acids are necessary and sufficient for Buc localization.............................. 23

1.5.3 The localization signal of Buc might contain prion-like domains ................................................ 24

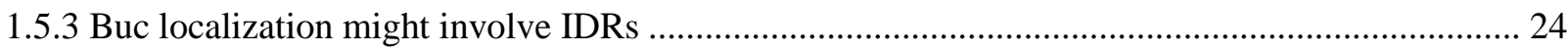

1.5.3 A role of Non-muscle myosin II in anchoring germ plasm ...................................................... 24

1.5.5 Buc is sufficient for primordial germ cell formation in embryos ............................................. 24

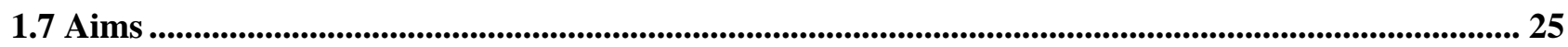

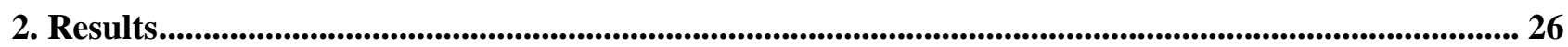

2.1 The role of tight junction proteins in Buc localization and degradation ............................................ 26 
2.1.1 Tight junction components co-localize with germ plasm during oogenesis and embryogenesis ... 26

2.1.2 Early embryo contains germ plasm specific and somatic ZO1 34

2.1.3 Phosphorylation of $\mathrm{ZO} 1$ is required for degradation of Buc 36

2.2 Bucky ball plays a role in germ plasm aggregation and localization 40

2.2.1 Buc is an intrinsically disorder protein and aggregates in-vitro 40

2.2.2 Dynamic nature of Bucky ball aggregation in vivo 40

2.2.3 $48 \mathrm{~N}$-terminal amino acids are sufficient for germ plasm localization. 45

2.2.4 Prion domains are not required for germ plasm localization 47

2.2.5 BucLoc phosphorylation is not required for germ plasm localization 55

2.3 Identification of the core BucLoc interactome 60

2.3.1 Identification of the core BucLoc interactome 60

2.4 Conserved functions of Bucky ball 66

2.4.1 Mechanism of primordial germ cells induction is conserved in vertebrates and invertebrates ......... 66

2.4.2 Germ plasm localization is conserved in vertebrates 68

3. Discussion 72

3.1 Functional relevance of Buc co-localization with cellular structure markers 72

3.1.1 The Balbiani body as a storage compartment for maternally provided cellular structure markers 72

3.1.2 Buc co-localizes with tight junction proteins during early embryogenesis. 73

3.1.3 The role of E-cadherin in germ plasm compaction 74

3.1.4 Functionality of Buc and ZO1 co-localization 75

3.2 Bucky ball plays a role in germ plasm aggregation and localization 77

3.2.1 PrDs in the BUVE motif of Buc might be responsible for germ plasm aggregation during oogenesis 77

3.2.2 Buc aggregates via its IDRs during embryogenesis 78

3.2.3 The prion-like domains of Buc are not required for germ plasm localization 79

3.3 The core BucLoc interactome. 81

3.4 Conserved functions of Buc 81 


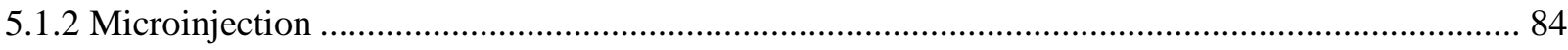

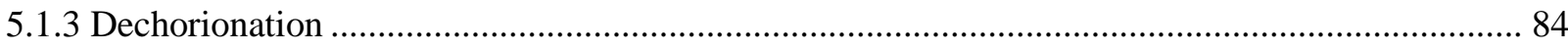

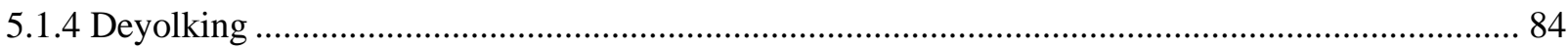

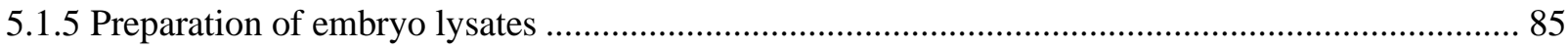

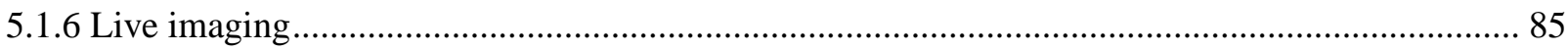

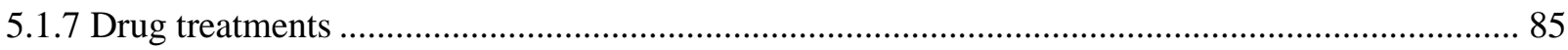

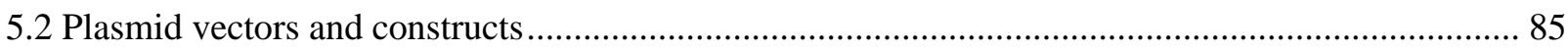

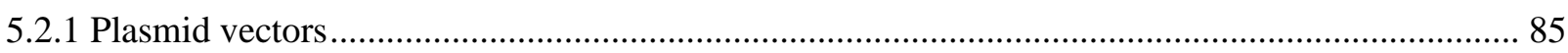

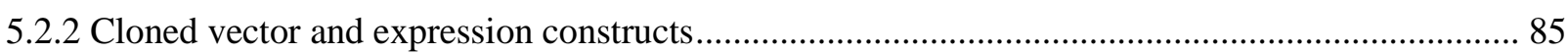

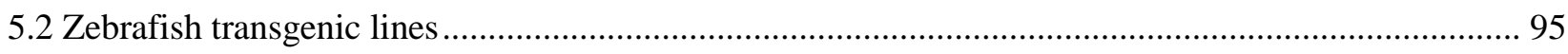

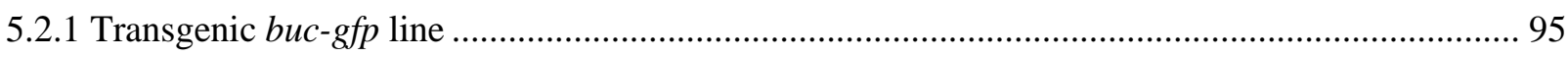

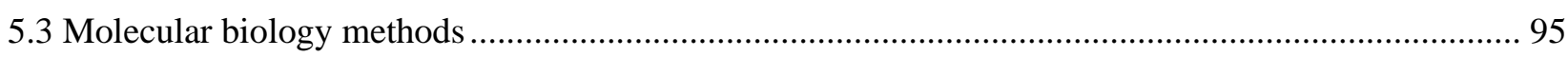

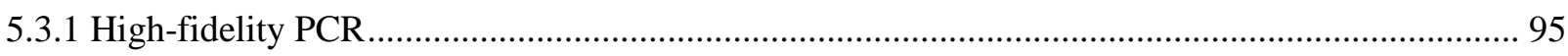

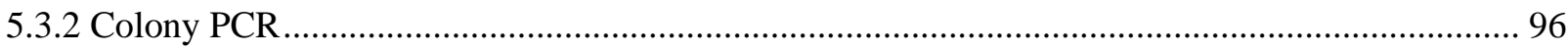

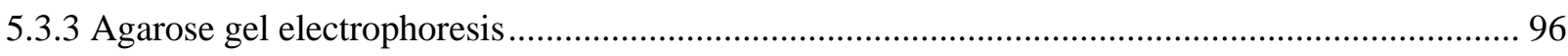

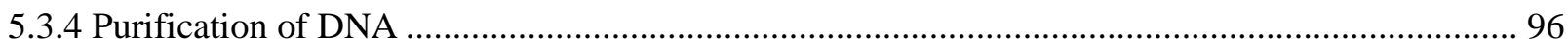

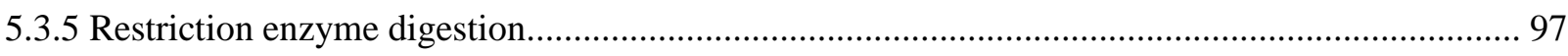

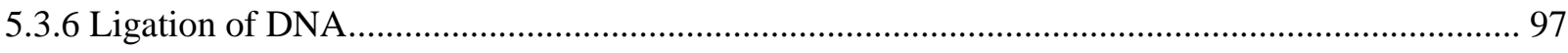

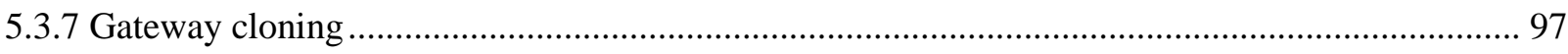

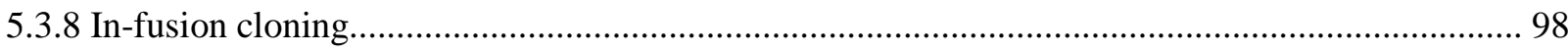

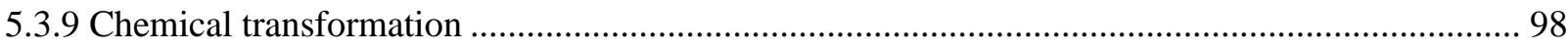

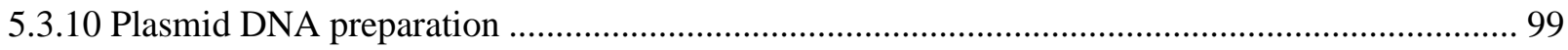

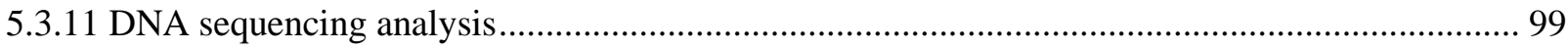

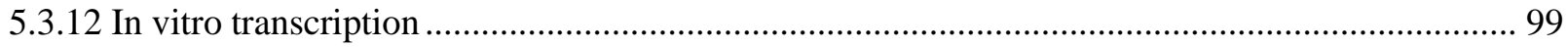




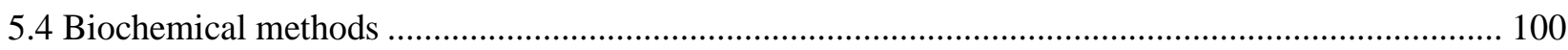

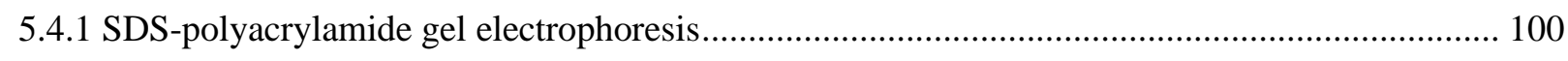

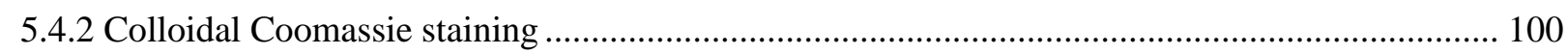

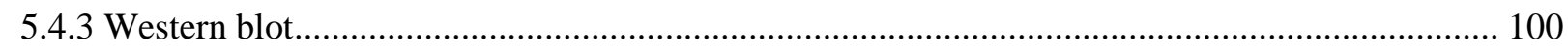

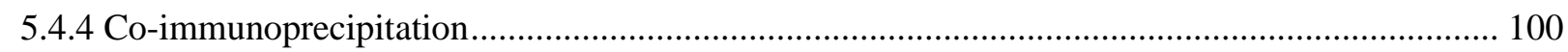

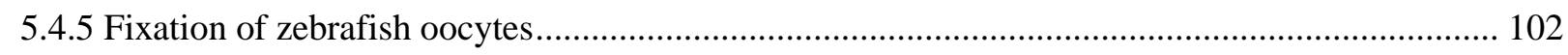

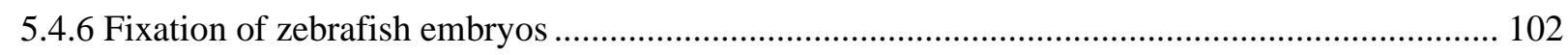

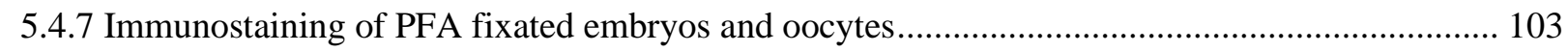

5.4.8 Immunostaining of glyoxal fixated embryos and oocytes ................................................... 103

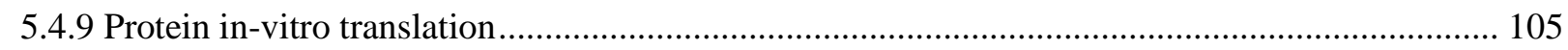

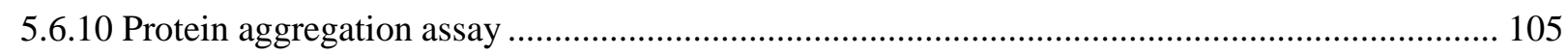

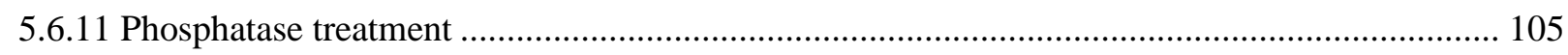

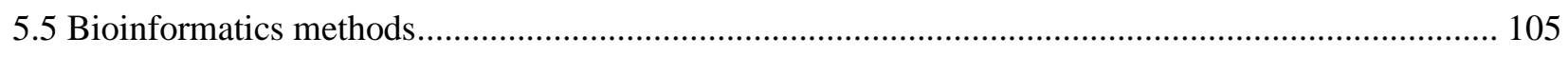

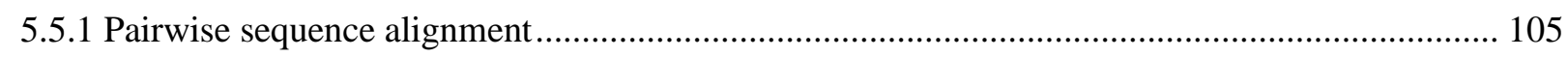

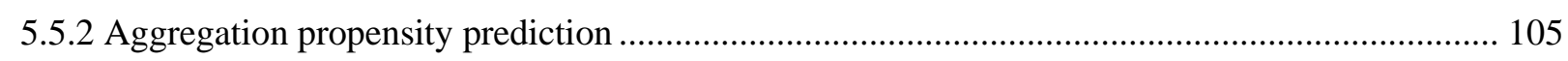

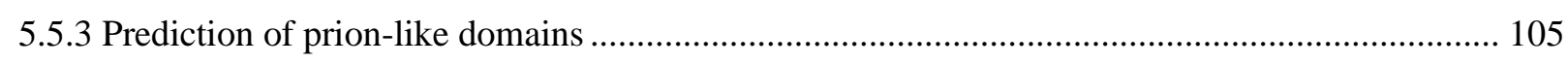

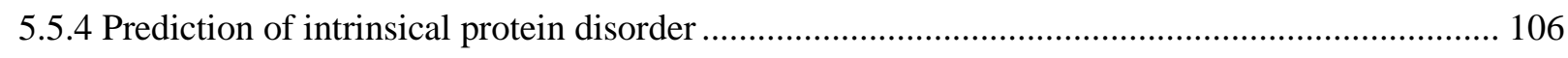

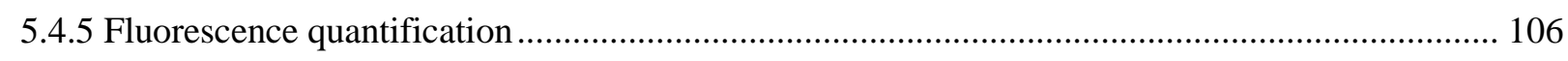

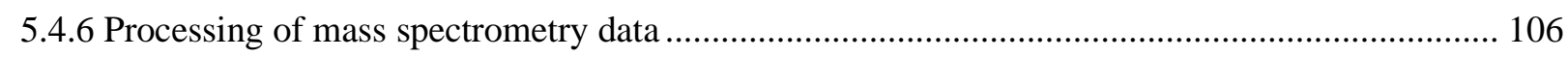

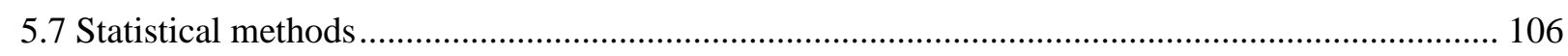

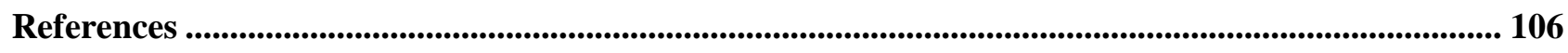

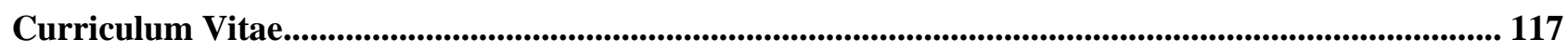




\section{Acknowledgments}

First of all, I am grateful to my supervisor Dr. Dosch for giving me an opportunity to work in his lab and providing the funding for my PhD research. Also, I want to thank Dr. Dosch for his active support and participation in my $\mathrm{PhD}$ project.

I thank the members of my thesis committee Prof. Dr. Fred Wouters and Prof. Dr. Herbert Jäckle for their scientific contribution to my PhD project.

I thank Dr. Julia Gross for making her cell culture facility available for my experiments and Mona Honemann-Capito for help with the use of the cell culture facility. Likewise, I thank Dr. Karen Linnemannstöns for sharing her experience with me about the use of the confocal laser scanning microscopy.

I also thank Prof. Dr. Jörg Großhans for giving me the opportunity to work on the confocal laser scanning microscopy device. Likewise, I thank Johannes Sattmann and other members of the research group of Jörg Großhans for helping me to use the microscopy device.

I thank Christof Lenz and his research team for performing the mass spectrometry experiments and helping me with data analysis.

I am grateful to my lab members Roshan Perera, Gudrun Kracht, Nadia Rostam, Hazem Kalifa and Pritesh Krishnakumar for their support during my work.

I am grateful to my colleagues from the Department of Developmental Biochemistry and Human Genetics for their help in conducting my $\mathrm{PhD}$ research.

I am also grateful for the financial support of GGNB for going to the scientific meetings and being able to conduct my research in the last phase of my PhD project. Also, I thank the GGNB team for their wiliness to help. 


\section{List of abbreviations}

\begin{tabular}{|c|c|}
\hline${ }^{\circ} \mathrm{C}$ & Degrees Celsius \\
\hline aa & Amino acids \\
\hline AJ & Adherens junction \\
\hline Balbiani body & $\mathrm{Bb}$ \\
\hline $\mathrm{bp}$ & Base pairs \\
\hline BSA & Bovine serum albumin \\
\hline $\mathrm{CC}$ & Calphostin C \\
\hline cDNA & Complementary DNA \\
\hline Co-IP & Co-immunoprecipitation \\
\hline C-terminus & Carboxy-terminus \\
\hline DAPI & 4',6-diamidino-2-phenylindole \\
\hline $\mathrm{dH} 2 \mathrm{O}$ & Distilled water \\
\hline DNA & Deoxyribonucleic acid \\
\hline dNTP & Deoxynucleotide triphosphate \\
\hline $\mathrm{dpf}$ & Days post fertilization \\
\hline EVL & Enveloping layer \\
\hline E. coli & Escherichia coli \\
\hline e.g. & Exempli gratia \\
\hline EDTA & Ethylenediaminetetraacetic acid \\
\hline eGFP & Enhanced green fluorescent protein \\
\hline et al. & Et alii \\
\hline FMA & Furrow microtubule array \\
\hline fw & Forward \\
\hline $\mathrm{g}$ & Gram \\
\hline GFP & Green fluorescent protein \\
\hline h & Hour \\
\hline 1.6-HD & 1,6-hexanediol \\
\hline HD & Hemidesmosome \\
\hline hpf & Hours post fertilization \\
\hline IP & Immunoprecipitation \\
\hline IDP & Intrinsically disordered protein \\
\hline IDR & Intrinsically disordered region \\
\hline $\mathrm{kb}$ & Kilo base pairs \\
\hline
\end{tabular}




\begin{tabular}{|c|c|}
\hline $\mathrm{kDa}$ & Kilodalton \\
\hline LB & Lysogeny broth \\
\hline M & Molar \\
\hline MB & Midbody \\
\hline MS & Mass spectrometry \\
\hline $\mathrm{mg}$ & Milligram \\
\hline $\min$ & Minute \\
\hline Mpf & Minutes post fertilization \\
\hline miRNA & Micro RNA \\
\hline $\mathrm{ml}$ & Millilitre \\
\hline MLCK & Myosin Light Chain Kinase \\
\hline $\mathrm{mM}$ & Millimolar \\
\hline mRNA & Messenger RNA \\
\hline $\mathrm{n}$ & Number \\
\hline ng & Nanogram \\
\hline $\mathrm{nl}$ & Nanolitre \\
\hline NMII & Non-muscle myosin II \\
\hline p-NMII & Phospho-myosin light chain II \\
\hline N-terminus & Amino-terminus \\
\hline ORF & Open reading frame \\
\hline PAGE & Polyacrylamide gel electrophoresis \\
\hline PBS & Phosphate-buffered saline \\
\hline PBT & Phosphate-buffered saline Triton X-100 \\
\hline PLAAC & Prion-Like Amino Acid Composition \\
\hline PLD & Prion-Like Domain \\
\hline PCR & Polymerase chain reaction \\
\hline pg & Picogram \\
\hline PGC & Primordial germ cell \\
\hline $\mathrm{pH}$ & Potentium hydrogenium \\
\hline piRNA & Piwi-interacting RNA \\
\hline PKC & Protein Kinase C \\
\hline PrDs & Prion Like Domains \\
\hline p-NMII & Phosphorylated non-muscle myosin II \\
\hline RLC & Regulatory Light Chain \\
\hline RNase & Ribonuclease \\
\hline
\end{tabular}


ROCK

rpm

RRM

RT-qPCR

TJ

$\mathrm{ZO}$
Rho Associated Protein Kinase

Rounds per minute

RNA recognition motif

Reverse transcription quantitative real-time PCR

Tight junction

Zonula occludens 


\section{Abstract}

Zebrafish germline is specified early during embryogenesis by inherited maternal RNAs and proteins called germ plasm. Only those cells containing germ plasm will become part of the germline, whereas other cells will commit to somatic cell fates. Therefore, proper localization of germ plasm is crucial for germ cell specification. In our lab we discovered the bucky ball (buc) gene. Buc is the first protein in vertebrates required for germ plasm aggregation and induction of primordial germ cells. Fascinatingly, Buc mirrors germ plasm localization during all stages of zebrafish embryogenesis and oogenesis. Hence, to investigate germ plasm localization, I used Buc as a molecular proxy. Previously, we mapped the localization signal of Buc and identified non-muscle myosin II (NMII) as one of the Buc interactors involved in germ plasm localization. However, the fundamental mechanisms responsible for germ plasm localization remain largely unexplored.

In this study, we analyzed various NMII-associated cellular structures for their role in germ plasm localization, using immunohistochemistry. Moreover, we did a fine-mapping and mutagenesis of the localization domain of Buc to understand the requirement of predicted protein aggregation domains in germ plasm localization. After the refined mapping, we utilized the identified Buc localization sequence to isolate proteins involved in germ plasm localization. Finally, we investigated whether the function of Buc in germ plasm localization is conserved in vertebrates and invertebrates by a combination of protein overexpression in vivo and immunohistochemistry.

We found that the cytoplasmic tight junction component Zonula occludens 1 isoform A (ZO1-A) colocalizes with Buc during oogenesis and embryogenesis. Furthermore, we demonstrated that ZO1 phosphorylation is required for Buc degradation. We also showed that Buc localization is mediated independently of the predicted aggregation domains. Additionally, we isolated 23 potential interactors with the Buc localization signal. In the end, we showed that the germ plasm organizer Xenopus Velo1 but not Drosophila short Oskar co-localizes with zebrafish germ plasm.

Previously, it was shown that germ plasm interacts with actin. However, how exactly germ plasm is anchored to cleavage furrow remained unknown. Our data indicate for the first time that germ plasm is anchored by tight junction, as early as at the 8-cell stage. However, it remains to be addressed if mature tight junctions are present during the first embryonic cleavages in zebrafish. We demonstrated a role of ZO1 phosphorylation in Buc degradation. Our results indicate that Protein Kinase C (PKC)mediated phosphorylation of ZO1 is required for the tight regulation of Buc levels during early embryogenesis. Our results also show that the predicted aggregation domains within the Buc localization signal are not required for germ plasm localization during early embryogenesis. This result is in line with a more liquid-like behavior of germ plasm during embryogenesis than during the oogenesis. Among the 23 potential interactors with the Buc localization signal, we identified Intracellular hyaluronan-binding protein 4 (Ihabp4) as the most promising candidate for anchoring germ plasm to cytoskeleton. Future study will focus on co-localization analysis of Buc and Ihabp4. In the end, our data shows that the molecular mechanism of germ plasm localization is conserved in vertebrates. We hypothesize, that Velo1 targets to zebrafish germ plasm also via its $\mathrm{N}$-terminal localization signal. Further understanding of molecular mechanisms of germ plasm localization might lead to a better understanding of germ cell formation and to the establish new drug targets and therapies against infertility. 


\section{Introduction}

A biological process resulting in creation of a unique organism is called reproduction. Sexual reproduction requires a specific subtype of cells called germ cells. In metazoa, germ cells are specified early during embryonic development (Irie \& Kuratani, 2014; Kumano, 2015). This early germ cells specification enables segregation of germ line from soma. With development of an organism, germ cells differentiate into primordial germ cells (PGCs), migrate to gonad region and establish the germline (Strome \& Updike, 2015). Therefore, development of germ cells is a multistep and complex process. This process ensures the propagation and survival of a species. However, the molecular mechanisms responsible for this important process remain poorly investigated.

A better understanding of the process of germ cell formation will help to identify causes for infertility and might lead the way to the identification of new drug targets for future therapies of such diseases. Furthermore, understanding of how germ cells are specified will help to understand specification of other cell types, not related to the germline.

\subsection{Mechanisms of germ cell specification}

There are two mechanisms by which the segregation of germline from soma is achieved: preformation and induction. The induction mechanism involves germ cells specification through external signals from surrounding somatic cells. This mode of germ cell specification is characteristic for mice and axolotl.

Preformation mechanism involves the inheritance of germ plasm, a localized egg cytoplasm containing cytoplasmic determinants (Figure 1) (Extavour \& Akam, 2003). Cytoplasmic determinants are maternal factors which are synthesized during oogenesis and localized to a specific compartment in oocytes. In the early embryo, cytoplasmic determinants asymmetrically segregate between blastomeres. The blastomeres with localized cytoplasmic determinants acquire a specific cell fate. Germ plasm acts like a cytoplasmic determinant and induces germ cells formation.

Despite the difference in germline specification mechanism between the induction and preformation, both germ cell specification mechanisms result in similar germ cell developmental programs (EwenCampen et. al., 2010; Extavour \& Akam, 2003; Juliano et. al, 2010). Therefore, preformation is an important and interesting process for research in vertebrates. 

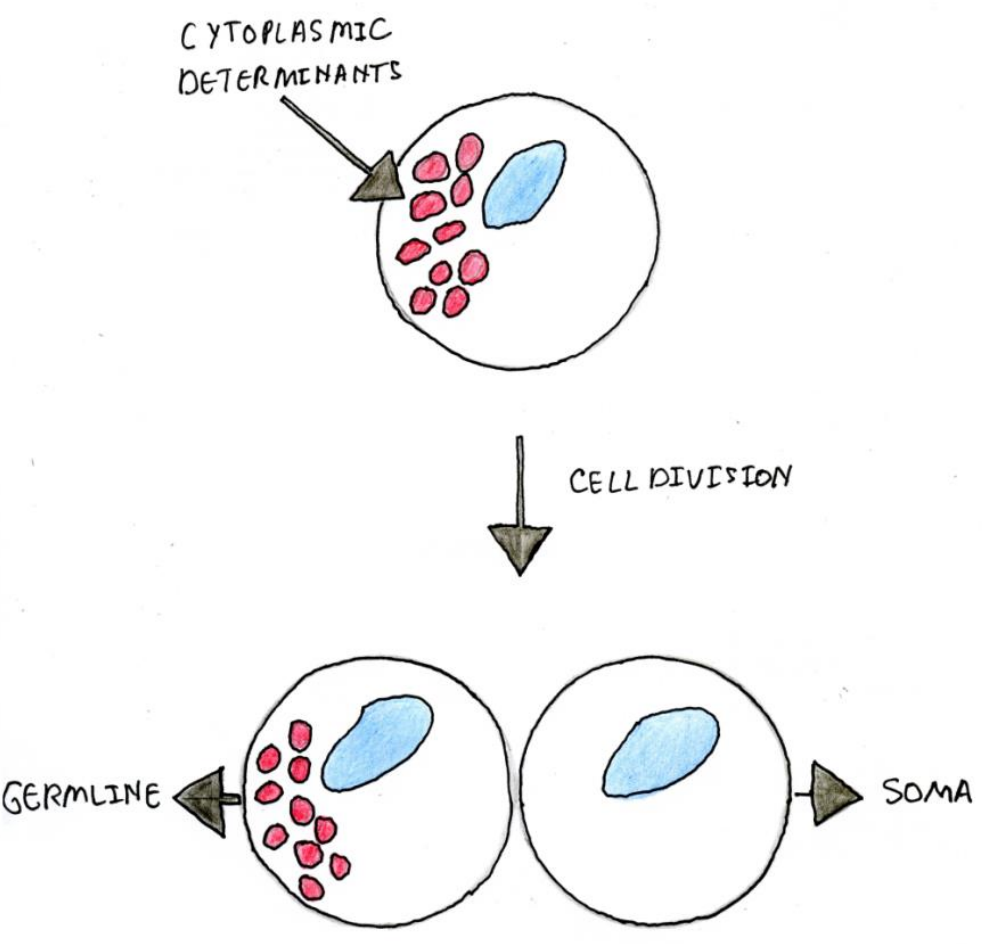

Figure 1: Germline is specified by cytoplasmic determinants. Cytoplasmic determinants reside in the cytoplasm of cells during early embryonic development. Upon cell division, a subset of cytoplasmic determinants (germ plasm) is asymmetrically localized to cells which will become germ cells. In contrast, the cells which will not get the germ plasm localized will develop into somatic cells.

\subsection{Germ plasm and its role in germ cell specification}

In vertebrates germ plasm consists of maternally inherited factors (Pelegri 2003). As these factors act like a cytoplasmic determinant, they are crucial for germ cells specification during early embryogenesis. Therefore, the process of germ cell specification can be separated in two phases. The first phase starts already during oogenesis, when the maternal factors are produced. The second starts during embryogenesis when germ cells specification starts.

\subsubsection{Germ plasm localizes to the Balbiani body during early oogenesis}

Balbiani body $(\mathrm{Bb})$ is an RNA granulum containing cytoplasmic determinants, which is localized in early oocytes (Kloc et al., 2004). The Bb consists of various RNAs and proteins, electron-dense granular/fibrous material, golgi, endoplasmic reticulum and a large number of mitochondria (Boke $e t$ al., 2016; Heasman et al., 1984; Huang et al., 1999; Lei \& Spradling, 2016). Hence, the Bb is also called mitochondrial cloud in Xenopus (Kloc et al., 2004). The Bb was first identified in oocytes of spiders in 1845 by von Wittich. Remarkably, it is present in almost all animal oocytes of invertebrates (e.g. spiders, insects and mollusks) and vertebrates (e.g. frogs, birds, teleosts and mammals) (von Wittich, 1845; Guraya, 1979; Kloc et al., 2004). The Bb is found even in the early oocytes of mammals such as mice and humans (Albamonte et al., 2013; Pepling et al., 2007). However, there is no explanation for the presence of $\mathrm{Bb}$ in mammals, as the latter specify germline by induction. In Xenopus $\mathrm{Bb}$ is believed to store and protect RNAs and healthy mitochondria during development in order to pass into the PGCs (Cox, 2003; Kloc et al., 2004; Kogo et al., 2011; Marinos \& Billett, 
1981). In Xenopus, Bb consists of protein aggregates. These aggregates form amyloid-like matrix, responsible for entrapping mitochondria and RNAs (Boke et al., 2016). However, it remains to be addressed if other organisms could also assemble $\mathrm{Bb}$ by the formation of amyloid-like matrix.

In zebrafish, $\mathrm{Bb}$ together with nucleus are the first markers of animal-vegetal (AV) polarity that will determine the anterior-posterior axis later on during embryogenesis (Escobar-Aguirre et al., 2017). Once the AV polarity of oocytes is established, it will be crucial for a proper distribution of germ plasm in an embryo and formation of germ cells. In zebrafish, the $\mathrm{Bb}$ aggregates at the vegetal cortex, adjacent to the nucleus of stage $\mathrm{Ib}$ oocytes. The $\mathrm{Bb}$ is linked with the oocyte cortex, where the $\mathrm{Bb}$ unpacks its mRNPs to specify the vegetal pole of the oocyte. The formation of the Bb precursor is initiated when telomeres of chromosomal bouquet associate with the centrosome (Elkouby et al., 2016). Furthermore, the formation of the $\mathrm{Bb}$ precursor involves microtubule dependent transport of mRNPs towards the telomere cluster cytoplasm (Elkouby et al., 2016). Then the Bb precursor components aggregate in a specialized nuclear cleft around the centrosome (Elkouby et al., 2016). During the stage II of oogenesis, the Bb disassembles into islands (Escobar-Aguirre et al., 2017). These islands move towards and are anchored at the vegetal cortex. During the stage I oogenesis, germ plasm components such as RNA are accumulated at the region of the Bb called the messenger transport organizer (METRO). This RNA localization is microtubule independent. Many of germ plasm RNAs like nanos, dazl and vasa localize to the Bb via the METRO pathway during the stage $\mathrm{Ib}$ oogenesis. Later during embryogenesis, these RNAs become enriched in germ plasm. In contrast, RNAs which localize after the Bb disassembly, move towards the vegetal pole via a microtubule dependent pathway (King et al., 1999). For instance, germ plasm RNA such as bruno-like localizes via the microtubule independent pathway.

Thus, the aggregation of $\mathrm{Bb}$ during oogenesis precedes the localization of germ plasm components to the specific subset of cells during embryogenesis.

\subsubsection{The role of phase-separation in the Balbiani body and germ plasm assembly}

Functional protein aggregates, like amyloids, play a crucial role in germ cells development in mouse and frog (Berchowitz et al., 2015; Boke et al., 2016). The Bb in Xenopus is an amyloid-like aggregate, which is characterized by a cross- $\beta$ structure (Boke et al., 2016). Zebrafish Bb is most likely also an amyloid-like structure (Kloc et al., 2004). However, the amyloid state of the Bb was not addressed in zebrafish. In contrast to the $\mathrm{Bb}$, zebrafish germ plasm during embryogenesis behaves like liquid droplets (Riemer et al., 2015). Hence, there is a difference in a biohphysical state of germ plasm during embryogenesis and oogenesis. A formation of the amyloid-like or liquid-like aggregates is often driven by proteins which undergo phase-separation (Brangwynne et al., 2009, 2011; Kroschwald et al., 2015).

During a phase separation a protein 'de-mixes' into a dilute and dense phase (Alberti et al., 2019). For instance, it is assumed that the dense phase creates the liquid-like compartment. The liquid-like assemblies can further convert into amyloid-like assemblies. A subset of proteins which undergos the phase separation often consists of intrinsically disordered regions (IDRs) and contains prion-like domains (PrDs) (Kato et al., 2012; Kroschwald et al., 2015). The IDRs are characterized by a low amino acid complexity. For instance prion-like proteins are enriched in glutamine, asparagine and 
serine (Alberti et al., 2019). Proteins containing IDRs are considered intrinsically disordered proteins (IDPs) when they contain a disordered stretch of at least 30 residues (Tompa, 2012). The PrDs also contain low complexety regions, which are similar to yeast prion proteins (Hennig et al., 2015).

The ability of IDPs to phase-separate is an important feature for the formation of membrane-less organeless like the $\mathrm{Bb}$ (Brangwynne et al., 2009; Boke et al., 2016). However, the role of IDPs in the assembly of zebrafish $\mathrm{Bb}$ or germ plasm remains unclear.

\subsection{Germline development in zebrafish}

\subsubsection{Germline development in zebrafish}

Aggregation of $\mathrm{Bb}$ and subsequent localization of germ plasm components during oogenesis is crucial for their correct localization to germ plasm during embryogenesis (Escobar-Aguirre et al., 2017). Localization of germ plasm components during the stage Ib oogenesis into $\mathrm{Bb}$ (Figure $2 \mathrm{~A} 1$ ), is followed up by $\mathrm{Bb}$ disassembly (Figure $2 \mathrm{~A} 2$ ). Localization of germ plasm shortly before and during first embryonic cleavages is best described for RNAs (Knaut et al. 2000, Theusch et al. 2006; Yoon et al, 1997). Upon egg fertilization, germ plasm RNAs translocate to the animal pole (Figure 2 B) and become localized within a wide band at the periphery of the blastodisc (Theusch et al., 2006). During the first embryonic cleavages, germ plasm becomes enriched at distal ends of the first two cleavage furrows (Figure $2 \mathrm{C}, \mathrm{D})$. Next, at the 32-cell stage (1.75 hpf) germ plasm aggregates ingress into four cells (Yoon et al., 1997) and at the 512-cell stage (2.75 hpf) germ plasm still localizes to the four cells (Figure $2 \mathrm{E}$ ) (Knaut et al., 2000; Yoon et al., 1997). This asymmetric distribution of germ plasm to the four cells induces formation of PGCs. Furthermore, it allows development of "germ plasm free" blastomeres into various cell types between 5 and $10 \mathrm{hpf}$ when cell lineages are formed (Kimmel et al., 1995). Then at the sphere stage (4 hpf), germ plasm spreads out through cytoplasm and is symmetrically distributed to both daughter cells (Figure 2 F) (Knaut et al., 2000; Yoon et al., 1997). This symmetric germ plasm distribution results in four clusters of PGCs. The symmetric distribution of germ plasm also overlaps with the midblastula transition, which begins at the 512-cell stage (2.75 hpf) and ends at the sphere stage (4 hpf). During the midblastula transition genomic DNA transcription is activated and some maternal RNAs are degraded by miRNA mediated decay and cells start to divide asynchronously (Kane et al., 1992, Giraldez et al., 2006). At the shield stage (6 hpf), PGCs clusters start migrating dorsally towards the shield, which is the zebrafish Spemann organizer (Figure $2 \mathrm{G}$ ). Furthermore, the cytoplasmic germ plasm localization changes to perinuclear (Braat et al., 2000; Voronina et al., 2011) During blastula period, from dome to epiboly stages (4.3 hpf and 5.3 hpf), the number of germ cells increases up to 30 per embryo (Yoon et al., 1997). Then as epiboly progresses, the four groups of vasa expressing cells migrate towards the dorsal side of the embryo and cluster in two groups on either side of the midline, maintaining the same distance to the right and left of the notochord. During the segmentation period (10-24 hpf) somites form, primary organs and the tail develop, and the embryo elongates (Kimmel et al., 1995). Then by $24 \mathrm{hpf}$, PGCs migrate towards the location where the yolk ball is connected to the yolk extension (Figure $2 \mathrm{H}$ ). Around this stage, the future gonad is populated by PGCs and somatic precursor cells (Braat et al., 2000; Raz, 2003).

After $24 \mathrm{hpf}$, the embryo has formed all important organs and matures within the next two days into a larva, ready to hatch (Kimmel et al., 1995). The larva reaches its juvenile stage at $30 \mathrm{dpf}$ and the 
fertile adult fish develops by $90 \mathrm{dpf}$. During the growth from juvenile to adult, PGCs differentiate into sperm or oocytes and the sex of the fish is specified. The next cycle of germ cell development will start when the fertile fish will mate and give rise to the next generation. Thus, during early zebrafish embryogenesis germ plasm is asymmetrically localized to the four cells, which will define the germline. However, the molecular mechanism of germ plasm localization to the four cells remains unknown.

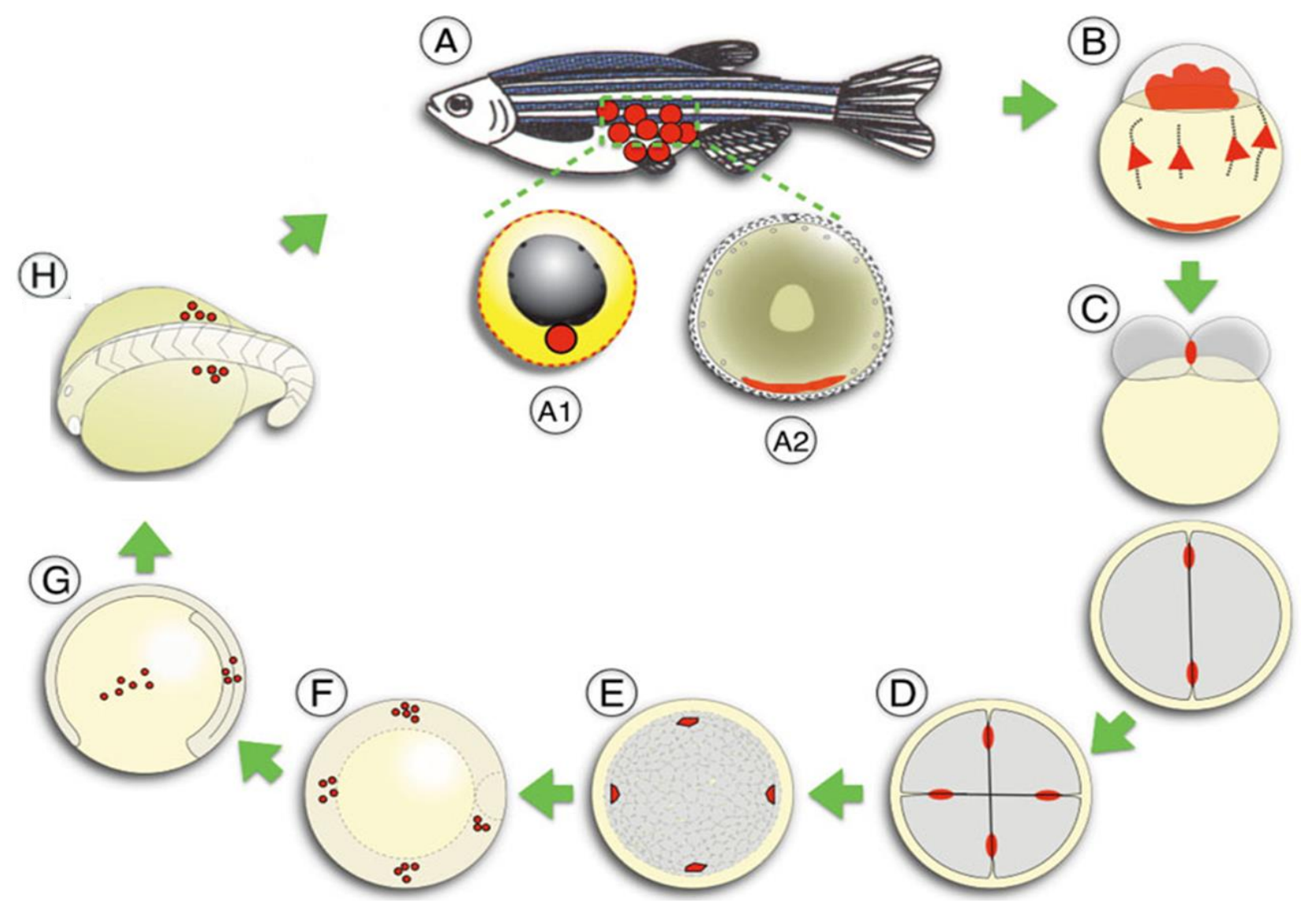

Figure 2: Germ plasm localization in zebrafish. Drawings represent different embryonic stages of zebrafish (A) development from early stage Ib oocytes (A1) until prim-16 stage (H) with the localized germ plasm (red). The yolk is drawn in yellow; orientation is indicated for each stage. Figure modified from (Dosch, 2015). 


\subsection{Molecular mechanisms of germ plasm localization during zebrafish embryogenesis}

Localization of germ plasm during first hours of zebrafish development is well described for germ plasm RNA components. However, the molecular mechanism required for localization of the four germ plasm aggregates remains highly unexplored.

\subsubsection{Role of cytoskeleton in early germ plasm aggregation}

Shortly after egg fertilization germ plasm RNAs, like vasa, localize in a wide cortical band at the animal pole, where they are associated with randomly oriented cortical f-actin (Figure $3 \mathrm{~A}, \mathrm{C}$ ) (Theusch et al., 2006). Then before the furrow formation, circumferential alignment of actin takes place and actin together with germ plasm RNAs are transported to periphery of an embryo (Figure 3 $\underline{\mathrm{B}, \mathrm{D})}$. Germ plasm movement to the periphery is mediated by astral microtubules. Aggregation and movement of germ plasm particles at the periphery can be prevented by treatment of embryos with microtubule destabilizing drugs (Theusch et al., 2006). Similarly, actin destabilizing drugs lead to reduction of germ plasm aggregates number (Theusch et al., 2006). Motley/birc5a mutants also show similar germ plasm aggregation defects as treatment of embryos with cytoskeletal drugs (Nair et al., 2013). Birc5a is a homolog of the mammalian Chromosomal Passenger Complex (CPC) and plays a role in various cellular processes (van der Waal et al., 2012). Birc5a co-localizes with germ plasm at the tips of astral microtubules (Figure 3 D) (Nair et al., 2013). Therefore it is hypothesized that Birc5a mediates microtubule-microfilament interaction and by this facilitates germ plasm aggregation and possibly recruitment to furrows (Nair et al., 2013). Mutants of another CPC component Aurora B Kinase also show that cytoskeleton is important for the recruitment of germ plasm to the furrow (Yabe et al., 2009). Furthermore, maternal mutants of the cytoskeletal regulator Mid1ip1L (MID1 interacting protein 1 like) show defects in germ plasm localization to early cleavage furrows (Eno et al., 2018b). Mid1ip1L is found as single puncta on cortical F actin (Figure $3 \mathrm{C}, \mathrm{D}$ ). It is required for reorganization of cortical F-actin in an early embryo, including the formation of circumferential arcs (Eno, 2016). Therefore it is hypothesized that cortical F-actin dynamics are not only responsible for germ plasm aggregation at the periphery of an embryo, but also for its recruitment to the furrow (Eno et al., 2018b). Also, localization of germ plasm to the cleavage furrow depends on proper cell division. Centriolar protein Sas-6 mutants have defects in second cell division, hence germ plasm does not localize any more in these mutants (Yabe et al., 2007).

\subsubsection{Kinesin-1 recruits germ plasm to cleavage furrows}

It has been shown that the maternally expressed Kinesin-1 (Mkif5Ba) is a binding partner of Buc (Campbell et al., 2015). Furthermore, Mkif5Ba is required to enrich Buc at cleavage furrows. However, how exactly Kinesin-1 transports and anchors Buc at cleavage furrows is unclear. 


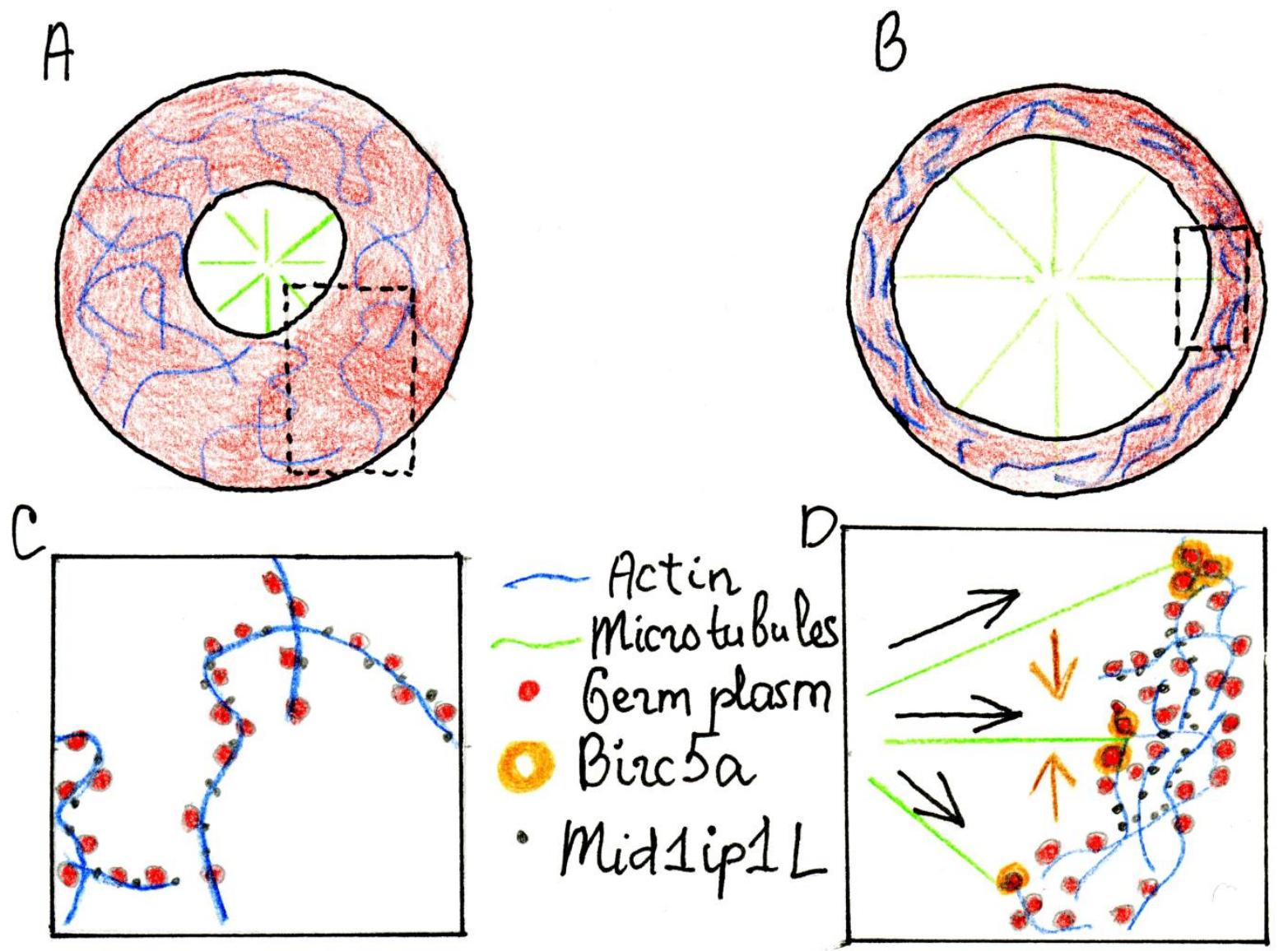

Figure 3: Role of cytoskeleton in early germ plasm localization. Germ plasm components (red) are initially bound in complexes to cortical filamentous actin (blue) (A-D). Astral microtubules (green) move these complexes to the periphery of blastomeres. This leads to germ plasm aggregation and recruitment to the forming furrow. (D) Birc5a co-localizes with germ plasm at the tips of astral microtubules (orange) and by this facilitates germ plasm aggregation. Mid1ip1L is found as single puncta on cortical $\mathrm{F}$ actin (black) and is believed to play a role in germ plasm aggregation and recruitment of germ plasm to the furrow. Black arrows indicate the movement of germ plasm to the cortex, whereas orange arrows germ plasm aggregation. Modified from: (Eno et al., 2018b; Nair et al., 2013; Theusch et al., 2006).

\subsubsection{Germ plasm compaction requires furrow microtubule array}

As soon as germ plasm is recruited to forming furrows, it undergoes a movement to distal ends of the cleavage furrows in a process called germ plasm compaction. At the 2-cell stage rod-like germ plasm aggregates are fully compacted into two symmetrical aggregates (Figure $4 \mathrm{~A}$ ). Upon furrow formation, the astral microtubules emanating from the spindle poles are substituted by an array or microtubules, parallel to each other and perpendicular to the furrow flanking the maturing furrow (Figure 4 A) (Jesuthasan, 1998; Danilchik et al., 1998). These latter microtubules are termed the furrow microtubule array (FMA). During furrow maturation, FMA is enriched at the distal ends of the cleavage furrow where it co-localizes with vasa RNA (Pelegri et al., 1999).

Maternal nebel mutants display defects in formation FMA and distal aggregation of vasa RNA (Pelegri et al., 1999). Furthermore, experiments with microtubule depolymerization drugs show similar defects as nebel mutants. Therefore, it is proposed that FMA is required for distal compaction of germ plasm.

An inhibition of calcium release or downstream mediators as Calmodulin also results in defective distal compaction of germ plasm (Eno et al., 2018a). Additionally, Nebel mutants show defects in 
enrichment of calcium stores and by this also in slow calcium waves (SCWs). Therefore, the proper germ plasm compaction by the FMA requires furrow associated SCWs (Figure 4).

FMA remodeling and disassembly has been also shown to require non-muscle myosin II (NMII) activity (Urven et al., 2006). NMII is a motor protein which co-localizes with germ plasm at the distal ends of cleavage furrows in zebrafish (Nair et al., 2013) (Figure 4 C). NMII structure includes two heavy chains, two essential light chains and two regulatory light chains (RLCs) (Figure 4 D). NMII becomes activated upon phosphorylation of the RLCs by various kinases including Rho-associated protein kinase (ROCK). This activation of NMII by the RLC phosphorylation induces NMII dependent assembly of myosin filaments and contractility. Pharmacological inhibition of the catalytic activity and downregulation of active NMII results in FMA defects. This supports the role of active NMII in germ plasm compaction. Pharmacological inhibitors of Rhoa and ROCK, applied during early zebrafish embryogenesis, induce similar germ plasm localization defects as in cei and nebel mutants or microtubule depolymerizing drugs (Miranda-Rodríguez et al., 2017; Pelegri et al., 1999; Yabe et al., 2009). Therefore, Rhoa/ROCK/NMII pathway is involved in germ plasm compaction.

A B

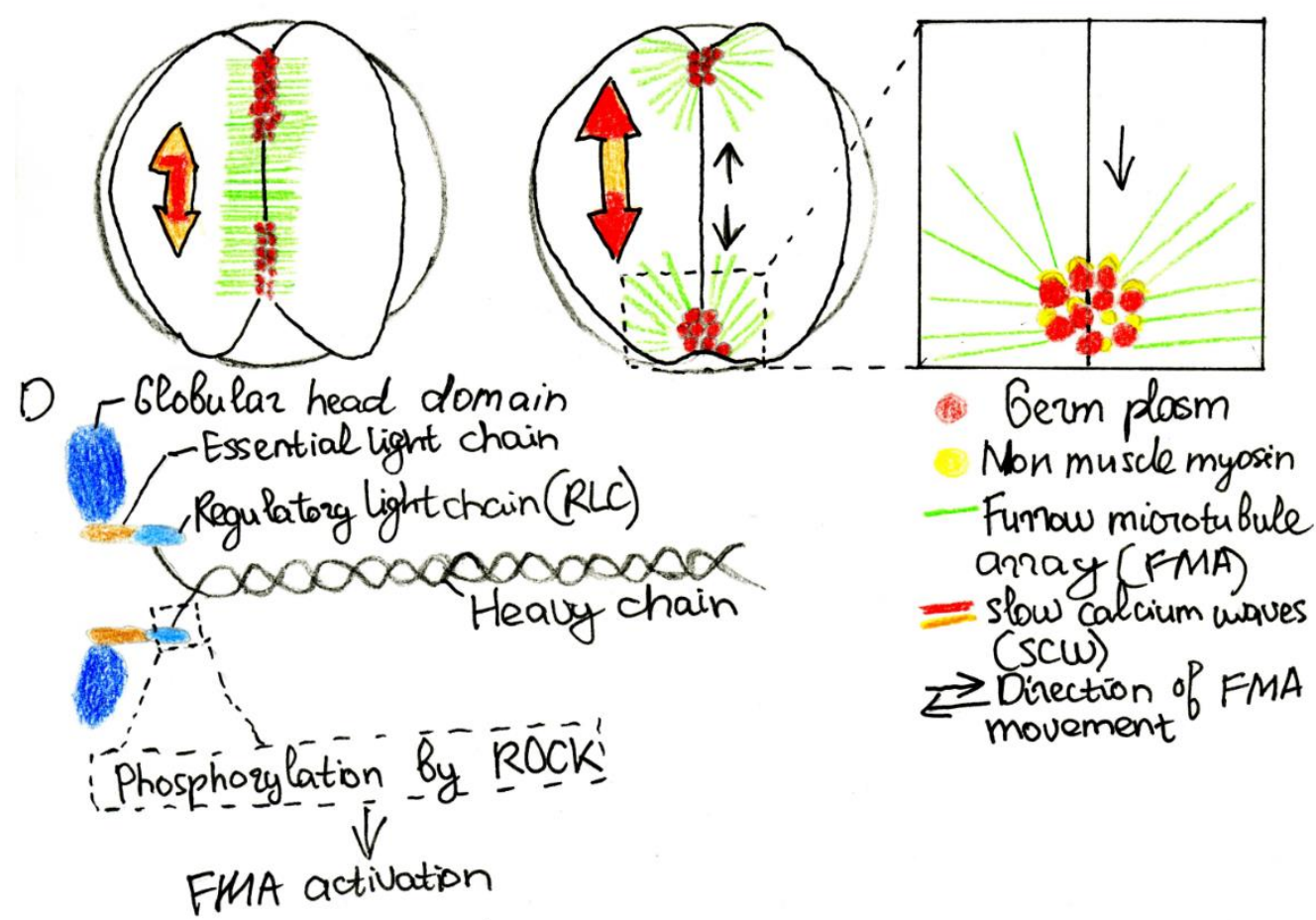

Figure 4: Germ plasm and FMA reorganization. (A) At the early two cell stage germ plasm forms rod-like aggregates along the cleavage furrow, whereas furrow microtubules (furrow microtubule array (FMA)) are arranged perpendicularly to the furrow. (A-B) During furrow maturation intracellular calcium (slow calcium waves (SCW)) travels in a medial to distal orientation (orange - weak SCW, red - strong SCW). (B-C) Upon the furrow maturation the germ plasm and FMA become enriched at the distal ends of the cleavage furrows. (C) At the distal ends of the cleavage furrows germ plasm (red) co-localizes with phosphorylated non-muscle myosin II (NMII) (yellow). (D) Structure and regulation of the NMII. NMII consists from two heavy chains (black), regulatory light chains (RLC, light blue), essential light chains (brown) and globular head domains (dark blue). RLC becomes phosphorylated by RhoA kinase (ROCK), activating ability of NMII to rearrange cytoskeleton. Subsequently, active NMII activates FMA and germ plasm compaction takes place. Modified from: (Eno et al., 2018a; Miranda-Rodríguez et al., 2017; Urven et al., 2006). 


\subsubsection{Asymmetric distribution of germ plasm is mediated by the spindle}

Following localization of the four germ plasm aggregates at the cleavage furrows, germ plasm is symmetrically segregated to the four cells (Knaut et al., 2000). Since the segregated germ plasm colocalizes with microtubules and one of the two centrioles, it is hypothesized that germ plasm segregation is mediated by the spindle apparatus (Knaut et al., 2000). This asymmetric segregation of germ plasm results in four primordial germ cells at the 1000-cell stage. Interestingly, nebel mutant embryos display germ plasm segregation defects, indicating that similar molecular process plays a role in translocation of germ plasm to the cleavage furrow and in germ plasm segregation or these both processes are interdependent. At the sphere stage, germ plasm starts to be symmetrically inherited by both daughter cells. However, the molecular mechanism underlying this change in localization is yet unknown.

\subsubsection{Localization of protein components of germ plasm}

One of the few proteins localizing to the germ plasm is Brul. Brul is localized to germ plasm until the 16-cell stage (Hashimoto et al., 2006). After this stage, Brul protein is distributed evenly through the cytoplasm. Ziwi is another germ plasm protein which localizes to the cleavage furrows of early stage embryos and to the perinuclear region in primordial germ cells at $24 \mathrm{hpf}$ (Houwing et al., 2007). Tudor domain-containing protein 6 a (Tdrd6a) localizes also to the germ plasm at the 4-cell stage and at $24 \mathrm{hpf}$ to germ cells (Roovers et al., 2018). Vasa protein localizes to the perinuclear region in primordial germ cells from $6 \mathrm{hpf}$ on (Braat et al., 2000). However, the localization mechanism of these proteins to germ plasm is yet unknown.

In conclusion, aggregation of germ plasm shortly before and during first embryonic cleavages depends on the interaction of cortical f-actin with germ plasm particles and translocation of these particles by microtubules to the cytokinetic ring at the base of the forming blastodisc (Theusch et al., 2006). This process requires proteins such as Birc5a and Mid1ip1L (Eno 2018b; Nair et al., 2013). Less is known about mechanisms of germ plasm proteins localization during early embryogenesis: how germ plasm is anchored to the furrows remains elusive (Theusch et al., 2006). When germ plasm is recruited to the furrow, it undergoes FMA dependent compaction to the distal ends of the cleavage furrows. This compaction depends on the action of NMII and Rhoa/ROCK pathway (MirandaRodríguez et al., 2017; Pelegri et al., 1999; Urven et al., 2006). The asymmetric inheritance of germ plasm to future PGCs is probably mediated by spindle microtubules (Knaut et al., 2000). Nevertheless, how germ plasm is symmetrically distributed to the daughter cells remains unknown.

\subsection{Bucky ball protein is a novel germ plasm component}

Protein composition of germ plasm remains largely unexplored. Therefore, there is a need for screening for proteins required for germ plasm related processes. A novel germ plasm gene bucky ball (buc) was discovered in the mutagenesis screen for maternal-effect mutations that control early vertebrate development (Dosch et al., 2004). Buc mutants (further referred as buc) show radial segregation of cytoplasm and do not develop beyond 1-cell stage (Figure 5). Therefore, non-polarized buc embryos resemble Buckminsterfullerene and hence are called bucky ball. 
wt

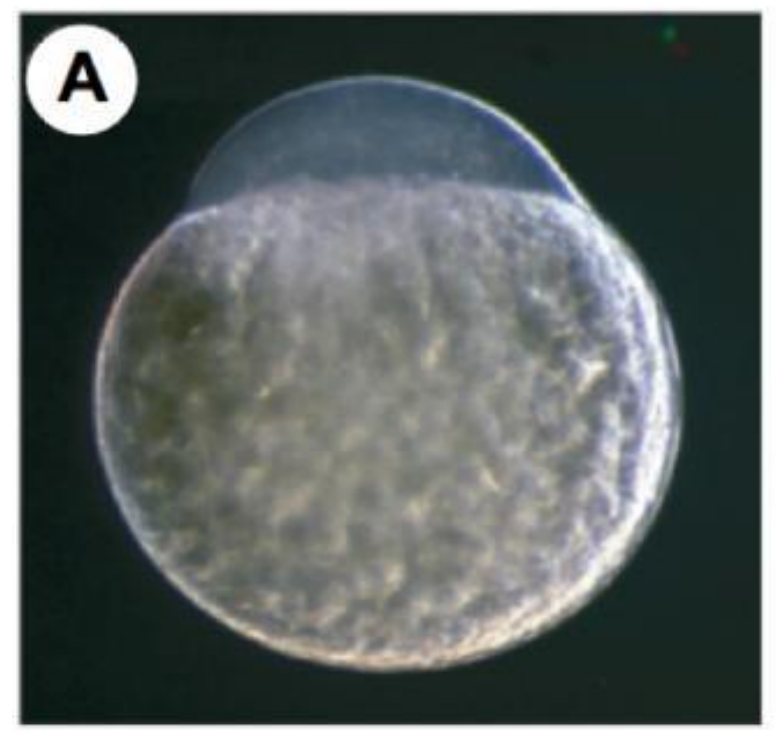

buc mutant

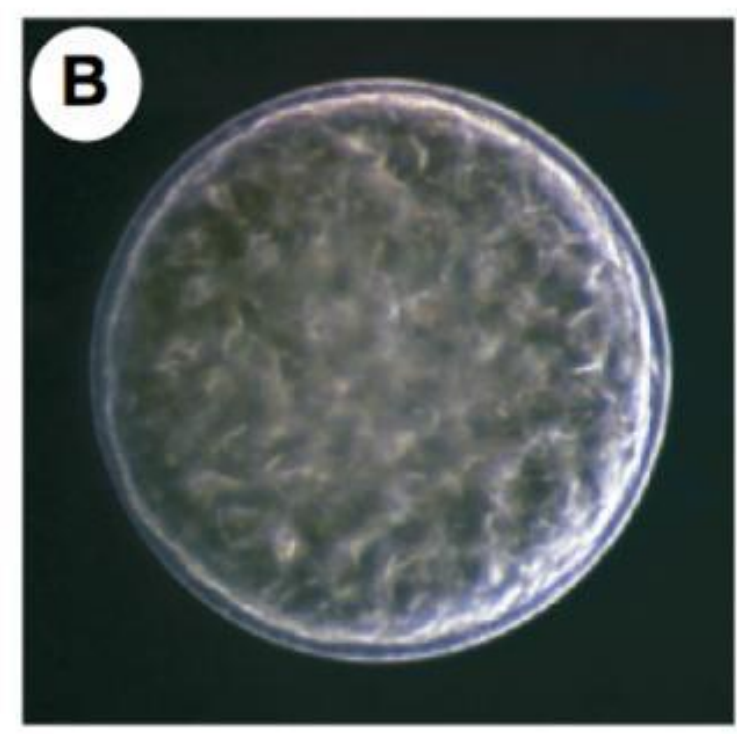

Figure 5: buc mutants show a defect in the embryonic animal-vegetal polarity. (A) The blastodisc is formed at the animal pole of WT embryo, whereas buc mutant embryo (B) shows a halo of cytoplasm surrounding the central yolk. Embryos at $30 \mathrm{mpf}$ are shown with animal pole to the top. Modified from (Dosch et al., 2004).

\subsubsection{Buc protein is required for germ plasm assembly and localization}

The localization of germ plasm components to the $\mathrm{Bb}$ during oogenesis precedes their inheritance by germ cells during embryogenesis (Escobar-Aguirre et al., 2017). Germ plasm RNAs such as vasa, nanos and dazl do not localize to the $\mathrm{Bb}$ in buc oocytes (Bontems et al., 2009). It was furthermore shown that Buc protein and not RNA is required for organization of germ plasm RNAs such as dazl during oogenesis (Bontems et al., 2009).

The germ plasm localization defects precede the loss of polarity in buc oocytes and embryos. Previously it was shown that the symmetry breaking events in oocyte are upstream of the Bb assembly by Buc (Elkouby et al., 2016). Hence, the Bb aggregation defects in buc oocytes are downstream of the $\mathrm{Bb}$ precursor formation via the microtubule-dependent mechanism (Chapter 1.2.1) (Elkouby et al., 2016).

During embryogenesis Buc localizes to germ plasm, mimicking the pattern of vasa RNA localization (Bontems et al., 2009; Yoon et al., 1997). In contrast to the Buc protein localization, buc RNA is distributed ubiquitously in early embryos. Hence, Buc protein is required for the $\mathrm{Bb}$ assembly, polarity maintenance and germ plasm localization in zebrafish (Bontems et al., 2009).

Positional cloning identified a gene responsible for $b u c$ phenotype. This gene encodes a novel protein with homologs among vertebrates: fish, amphibians, bird and mammals. Based on cDNA analysis from buc oocytes, Buc mutants were predicted to have a deletion of $277\left(\mathrm{Buc}^{\mathrm{p} 43}\right)$ and $37\left(\mathrm{Buc}^{\mathrm{p} 106}\right) \mathrm{C}$ terminal amino acids respectively (Bontems et al., 2009).

Studies on Buc homologs give a further understanding of its molecular mechanism of localization to germ plasm and the Bb assembly (Boke et al., 2016; Claußen \& Pieler, 2004; Škugor et al., 2016). Xenopus homolog of Buc, named Velo1, is described in a screen for vegetally localized RNAs in oocytes (Claußen \& Pieler, 2004). Velo1 protein localizes to Bb of Xenopus oocytes and is required for Bb assembly (Boke et al., 2016). Additionally, Atlantic Salmon homolog of Buc localizes during 
oogenesis and embryogenesis similarly to zebrafish Buc (Škugor et al., 2016). Hence, the function of Buc protein in germ plasm assembly and localization seem to be conserved in other species.

Thus, Buc is the first protein in vertebrates required for the $\mathrm{Bb}$ formation and localization of germ plasm components. Hence, Buc provides us a molecular proxy which can be used to study molecular mechanisms of germ plasm localization.

\subsection{2. $77 \mathrm{~N}$-terminal amino acids are necessary and sufficient for Buc localization}

In order to find out presence of conserved protein domains in Buc, multiple sequence alignment of 15 Buc related proteins was performed (Bontems et al., 2009). This analysis revealed two highly conserved motifs (aa 24-84, 114-128), located within the so called BUVE-motif (Buc-Velo) (Figure 6) (Bontems et al., 2009). Structure to function analysis of Buc identified $77 \mathrm{~N}$-terminal aa within the BUVE-motif (Buc11-88) which are necessary and sufficient for germ plasm localization (Riemer 2014, Doctoral dissertation).

Next to the conserved N-terminal domain of Buc, a highly conserved C-terminal domain (aa 372394) was identified (Figure 6) (Bontems et al., 2009). However, the function of this domain is not yet clear.

Thus, the localization signal of Buc is the first protein domain that targets to germ plasm in vertebrates. However, the molecular mechanism of Buc localization via the BUVE-motif remains unclear.

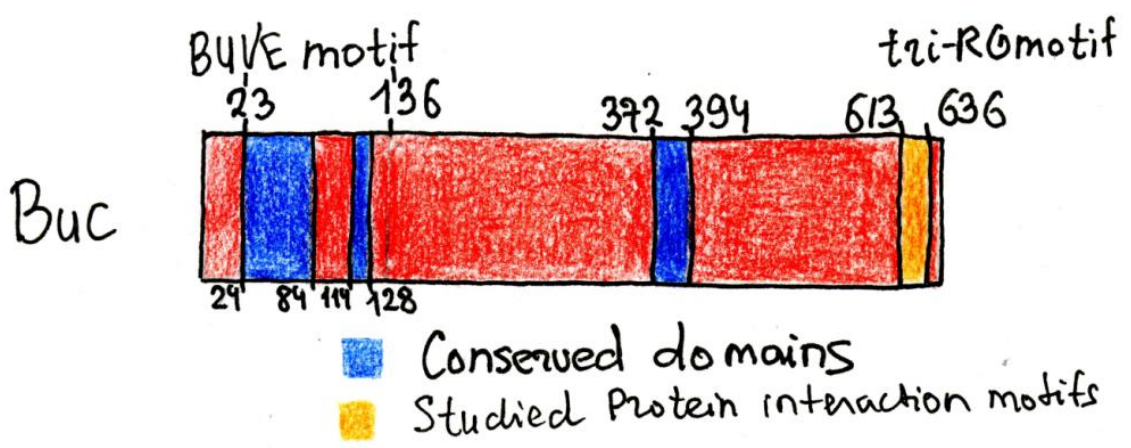

Figure 6: Conserved domains and functional protein interaction motifs of Buc. Schematic representation of Buc protein sequence including conserved domains (blue) and studied protein interaction motifs (orange). Figure is modified from: Riemer 2014, Doctoral dissertation and Roovers et al., 2018. 


\subsubsection{The localization signal of Buc might contain prion-like domains}

Previously, it was shown that Buc targets to and aggregates in Xenopus Bb. It was hypothesized that this aggregation behavior of Buc is driven via PrDs, located within the BUVE motif (Boke et al., 2016). Buc homolog in Xenopus Velo1 carries two PrDs in its BUVE motif (Boke et al., 2016). These PrDs are necessary for Velo1 aggregation to the Bb. However, exact locations and sequences of potential PrDs in Buc were not identified, as well as their requirement for Buc aggregation. The presence of the aggregation-prone PrDs in the BUVE-containing localization signal of Buc challenges our view on that germ plasm localization is mediated via direct protein-protein interactions.

\subsubsection{Buc localization might involve IDRs}

Previously it was shown that Velo1 is an IDP, which is in line with its ability to form amyloid-like, membraneless compartments like the Bb (Chapter 1.2.2) (Boke et al., 2016). Moreover, the BUVE motif of Velo1 is intrinsically disordered (Boke et al., 2016). Previously, Buc was proposed also to encode an IDP (Jeske et al., 2015). However, it remains unclear whether Buc contains IDRs in its localization domain and whether IDRs contribute to germ plasm localization.

\subsubsection{A role of Non-muscle myosin II in anchoring germ plasm}

To understand which proteins interact with Buc and by this could anchor germ plasm to cytoskeleton, a biochemical screen was performed using the localization signal of Buc (Riemer 2014, Doctoral dissertation). Remarkably, the biochemical screen identified a subset of non-muscle myosin (NMII) proteins, including multiple regulatory light chains (RLCs). Furthermore, we showed for the first time that Buc and phosphorylated NMII (p-NMII) at RLC co-localized during zebrafish oogenesis and embryogenesis (Riemer 2014, Doctoral dissertation). P-NMII plays a role in germ plasm compaction (Miranda-Rodríguez et al., 2017; Pelegri et al., 1999; Urven et al., 2006). Next to this function, our data suggests a role of $\mathrm{p}$-NMII in anchoring germ plasm to cytoskeleton. However, how exactly pNMII anchors Buc is unclear.

\subsubsection{Buc is sufficient for primordial germ cell formation in embryos}

Next to the role of Buc in germ plasm assembly and localization, Buc is required for induction of PGCs formation (Bontems et al., 2009). Upon overexpression of Buc in somatic cells, these cells acquired identity of PGCs. Interestingly, it seems like some functions of Buc are shared between other vertebrates. Velo1 and Buc share the ability to target to and assemble Bb in Xenopus (Boke et al., 2016). However, whether the PGC-inducing and germ plasm localization activities of Buc are shared between other vertebrates or invertebrates remains unknown.

Thus, Buc is the first protein in vertebrates required for germ plasm assembly and localization. Additionally, overexpression of Buc alone is sufficient to induce formation of ectopic PGCs. Buc homologs are present in other species and their functional studies point on the conserved role of Buc in the germ plasm organizing activities. The BUVE-containing N-terminal domain of Buc is required for germ plasm localization. However, the molecular mechanism of localization of Buc via the BUVE-containing motif remains unknown. Possibly it involves an interaction with p-NMII. 


\subsection{Aims}

Since the discovery of the four germ plasm aggregates in zebrafish, the molecular mechanism of germ plasm localization remains unexplored (Yoon et al., 1997). With the discovery of Buc, we have the first protein in vertebrates which alone targets to germ plasm (Yoon et al., 1997; Riemer et al., 2015). Hence, we use Buc as a molecular proxy to study the molecular mechanisms of germ plasm localization. In my doctoral dissertation I aimed to focus on following tasks:

\section{Identification of cellular structure that anchors Buc to cytoskeleton}

Previously, we isolated protein interactors of Buc. By this we have identified p-NMII as one of the promising candidates for anchoring germ plasm to cytoskeleton (Riemer 2014, Doctoral dissertation). P-NMII is known for its interaction with various cellular structures (Vicente-Manzanares et al., 2009). Hence, I am planning to identify a cellular structure which anchors Buc to cytoskeleton.

\section{Studying the regulation of Buc localization}

Understanding how Buc is anchored to cytoskeleton will give me a clue about signaling molecules acting upstream of germ plasm localization. Hence, I am planning to identify how germ plasm localization is regulated at the level of the cellular structure which anchors Buc to cytoskeleton.

\section{Refined mapping of Buc localization signal}

The isolation of Buc localization signal gives us a tool to study molecular mechanisms of germ plasm localization. However, it remains unclear which protein domains, residues or post-translational modifications are required for germ plasm localization. Especially, it remains unclear whether PrDs and IDRs are involved in germ plasm localization. Hence, I am planning to perform a refined mapping and mutagenesis of Buc localization signal to address these questions.

\section{Performing a refined biochemical screen to isolate proteins involved in germ plasm localization}

Despite the isolation of some Buc interactors with a promising function in germ plasm localization, a relatively long candidate list makes the selection and investigation of the candidate proteins time laborious (Riemer 2014, Doctoral dissertation). Hence, there is a requirement of a refined biochemical screen. Ideally, this refined screen will result in a lesser number of candidate proteins. Such sophisticated biochemical screen can be achieved by using a smaller Buc localization signal and/or different experimental conditions and controls. Therefore, I aim to perform the refined biochemical screen to isolate the most important proteins involved in germ plasm localization.

\section{Understanding evolutionary conservation of germ plasm localization}

We previously demonstrated that the BUVE motif of Buc is conserved among a broad range of organisms (Bontems et al., 2009). This result raises a question whether germ plasm localization via a specific protein domain is a common feature of organisms which specify their germ cells by 
preformation. In order to answer this question, we plan to investigate localization of germ plasm organizers Drosophila Oskar and Xenopus Velo1 in zebrafish.

\section{Results}

\subsection{The role of tight junction proteins in Buc localization and degradation}

Since the discovery of the four spots to which germ plasm localizes, the molecular mechanism of germ plasm localization to these spots remains largely unknown (Yoon et al., 1997). So far it has been shown that actin and microtubules are important for aggregation and translocation of germ plasm RNAs to the cleavage furrow during first embryonic cleavages. Furthermore, the translocation of germ plasm RNAs to the cleavage furrow involves proteins such as Birc5a and Mid1ip1L (Eno et al., 2018b; Nair et al., 2013). At the cleavage furrow, germ plasm co-localizes with microtubules which are part of the FMA, required for germ plasm compaction (Pelegri et al., 1999). However, how germ plasm is anchored only at the first two cleavage furrows remains elusive (Theusch et al., 2006).

\subsubsection{Tight junction components co-localize with germ plasm during oogenesis and embryogenesis}

Previously, it was also demonstrated that p-NMII co-localizes with Buc through oogenesis and embryogenesis (Riemer 2014, Doctoral dissertation). It is known that p-NMII is a part of circumferential actin belt associated with tight- and adherens junctions (Liu et al., 2012). Furthermore, NMII can act via actin to bring adherens junction and hemidesmosome components in close proximity and by this facilitate their function (Vicente-Manzanares et al., 2009). Also, p-NMII is a midbody component and is required for generation of constriction sites for subsequent abscission (Wang et al., 2019). Therefore, co-localization of p-NMII with germ plasm implies on a role of these cellular structures in anchoring germ plasm to cytoskeleton.

To address this hypothesis, we studied co-localization of Buc with selected markers for each of the cellular structures by immunohistochemistry. We expected to find a cellular structure marker colocalizing with Buc at the 8-cell stage. At this stage, germ plasm is only present at distal ends of first two cleavage furrows, while $3^{\text {rd }}$ and $4^{\text {th }}$ cleavage furrows do not get germ plasm localized. To investigate whether a cellular structure continuously co-localizes with germ plasm, we investigated stage $\mathrm{Ib}$ oocytes and 512-cell stage embryos for co-localization with Buc.

For tight junction we selected Zonula occludens ( $\mathrm{ZO}$ ) proteins 1 and 2 as markers for the colocalization analysis. ZO is a scaffold protein that links various tight junction and adherens junction components to cytoskeleton (Anderson et al., 1993). Interestingly, ZO1 co-localized with Buc during stage IB oogenesis (Figure 7 A). At the 4 cell stage, we found $\mathrm{ZO} 1$ and Buc co-localized along the entire length of furrows (Figure $8 \mathrm{C}, \mathrm{D})$. In contrast, at the 8-cell stage, one fraction of ZO1 colocalized with Buc, while the other fraction remained localized along the entire length of the furrow (Figure $8 \mathrm{~A}, \mathrm{~B}$ ). Interestingly, we also found that Buc granules still contain ZO1 at the 512-cell stage (Figure $12 \mathrm{~A}, \mathrm{~B})$. In contrast to $\mathrm{ZO} 1, \mathrm{ZO} 2$ did not co-localize with Buc during oogenesis or embryogenesis (Figure $7 \mathrm{~B}$, Figure $12 \mathrm{C}, \mathrm{D}$ ). Thus, $\mathrm{ZO} 1$ is a promising candidate for anchoring germ plasm to cytoskeleton.

Next to the tight junction markers, we tested adherens junction markers E-cadherin and afadin for colocalization with Buc. Interestingly, we also found E-cadherin and afadin co-localized with Buc in 
the $\mathrm{Bb}$ (Figure $7 \mathrm{D}, \mathrm{E})$. In contrast to oogenesis, at the 8-cell stage Buc and E-cadherin localized to the two separate domains at the furrow (Figure 9). E-cadherin localized along the proximal ends of the $1^{\text {st }}$ and $2^{\text {nd }}$ furrows, whereas Buc localized to the distal part of these furrows. Furthermore, at the 512-cell stage we found E-cadherin localization exclusively at cell-cell junctions (Figure $13 \mathrm{E}, \mathrm{F}$ ). This result is similar to E-cadherin localization pattern at the sphere stage (Tay et al., 2010). Furthermore, opposing to ZO1, neither E-cadherin nor afadin localized to Buc granules (Figure $13 \mathrm{~A}$, $\underline{B}, \mathrm{E}, \mathrm{F})$. Thus, adherens junction is unlikely to play a role in anchoring germ plasm to cytoskeleton during early embryogenesis.

We also tested midbody marker Kif23 for co-localization with Buc. In contrast, to E-cadherin or ZO1, Kif23 did not localize in $\mathrm{Bb}$ (Figure $7 \mathrm{G}$ ). Furthermore, the largest fraction of Kif23 localized to cytoplasm of dividing blastomeres at the 8-cell stage and did not co-localize with Buc (Figure 10). The pattern of cytoplasmic Kif23 localization resembles that of spindle microtubules at late telophase and is consistent with Kif23 function during cytokinesis (Lee et al., 2012). Kif23 also weakly localized along all cleavage furrows (Figure 10 A', B'), which is consistent with the previous result of Ki23 localization at the 8-cell stage in zebrafish (Chen et al., 2002). Hence, we assumed that at the 8 -cell stage Kif23 localization partially overlaps with the germ plasm localization. However, as the majority of Ki23 is localized to cytoplasm, we concluded that midbody does not play a role in anchoring germ plasm to cytoskeleton.

Next we checked the hemidesmosomal marker Intergin $\alpha 5$ (Int $\alpha 5)$ for co-localization with Buc. Int $\alpha 5$ forms a heterodimer with Integrin $\beta 1$ to form a primary receptor for the extracellular matrix protein fibronectin (Jülich et al., 2005). Int $\alpha 5$ co-localized with Buc in the Bb, similarly to ZO1 and Ecadherin (Figure 7 E). However, Inta5 did not localize with Buc at the 8-cell stage, but showed ubiquitous distribution among blastomeres (Figure 11). Later, during 512-cell stage we found integrin localized as single puncta (Figure $13 \mathrm{C}, \mathrm{D}$ ). This result is consistent with previous data of Int $\alpha 5$ localization during early zebrafish embryogenesis (Fitzpatrick et al., 2014). Therefore, we concluded that Int $\alpha 5$ also does not play a role in germ plasm localization.

Taken all results together, we concluded that $\mathrm{Bb}$ stores cellular structure components, followed by their release during embryogenesis. Fascinatingly, only the tight junction marker ZO1 co-localized with germ plasm during early embryogenesis. Therefore, tight junction might anchor germ plasm to cytoskeleton, while the other cellular structure markers play a role in development of somatic cells. 


\section{Tight junction}

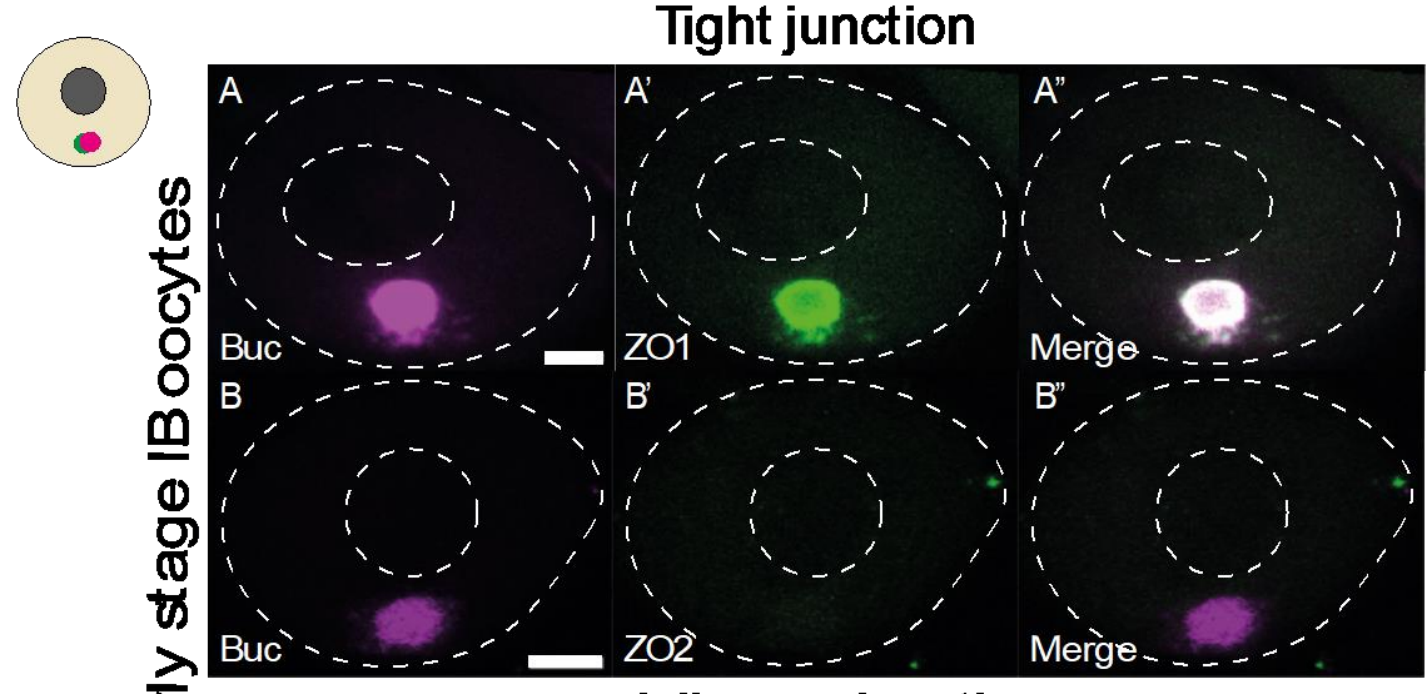

든

\section{Adherensjunction}

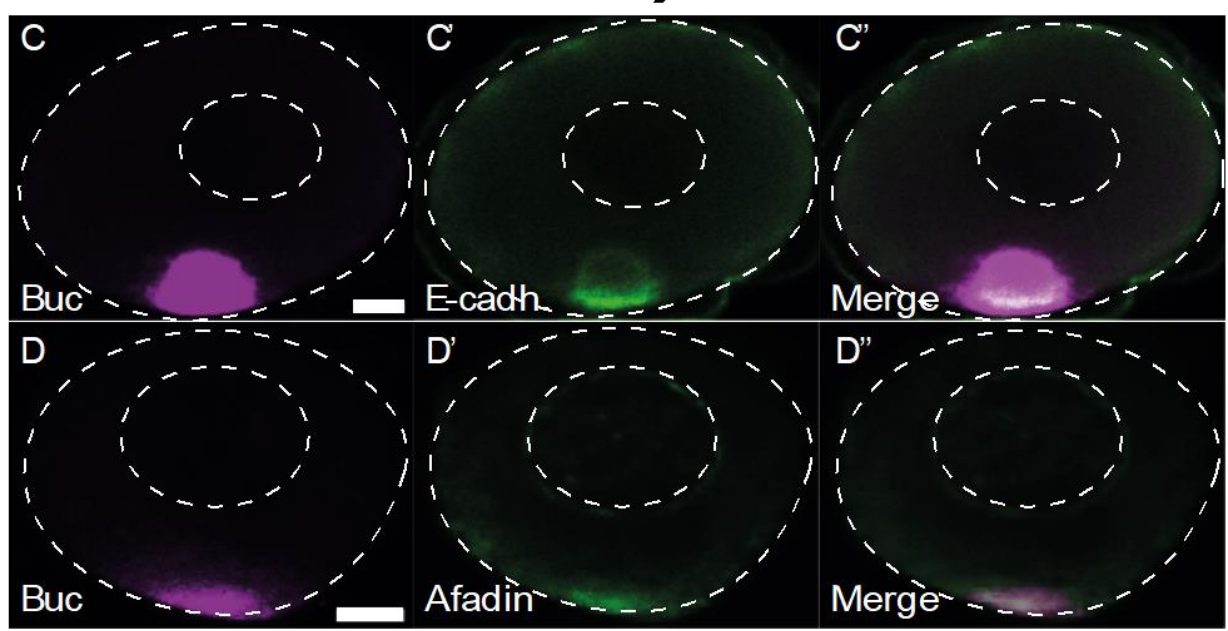

\section{Hemidesmosome}

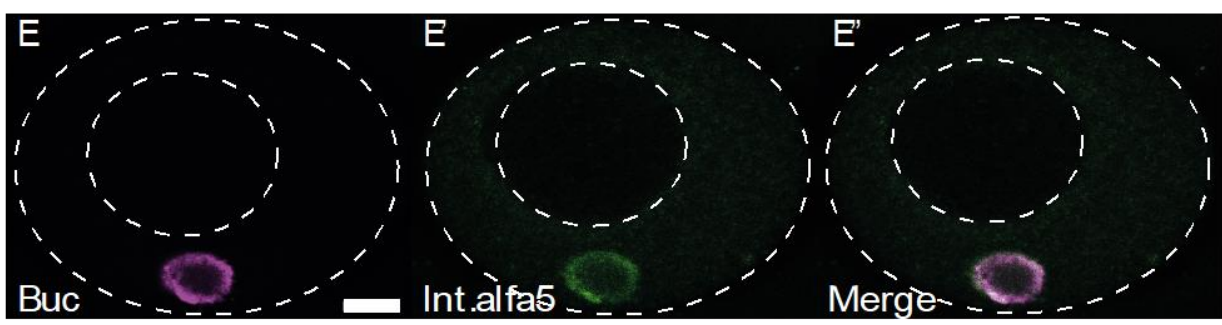

Midbody

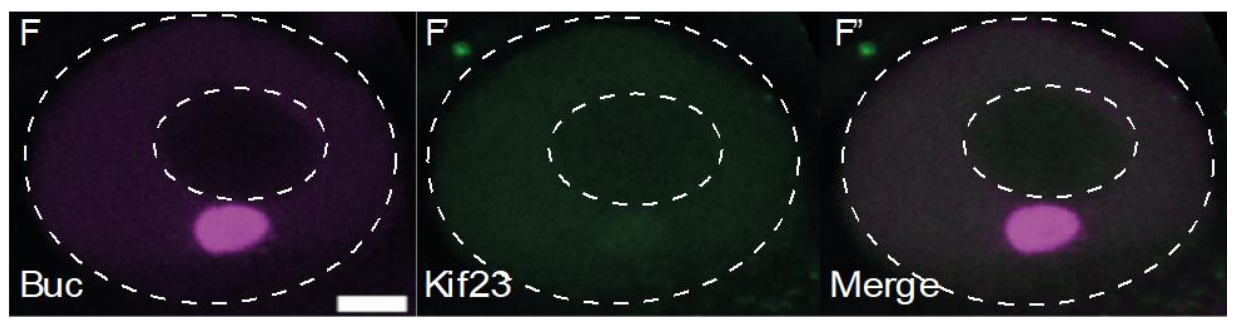

Figure 7: Cellular structure markers co-localize with Buc during oogenesis: Co-localization of Buc with different cellular structure markers during stage IB oogenesis was determined by immunohistochemistry: $1^{\text {st }}$ column - Buc (magenta), $2^{\text {nd }}$ column - cellular structure markers (green) and $3^{\text {rd }}$ column - merge. Buc co-localizes with tight junction markers ZO1 (A-A'), but not with ZO2 (B-B'). Furthermore, Buc co-localizes with adherens junction markers Ecadherin (C-C') and afadin (D-D'). Additionally, hemidesmosomal marker integrin alfa 5 co-localizes with Buc (E-E'). In contrast, midbody marker Kif23 does not co-localize with Buc (F-F'). Scalebars: $5 \mu \mathrm{m}$. 


\section{Tight junction}

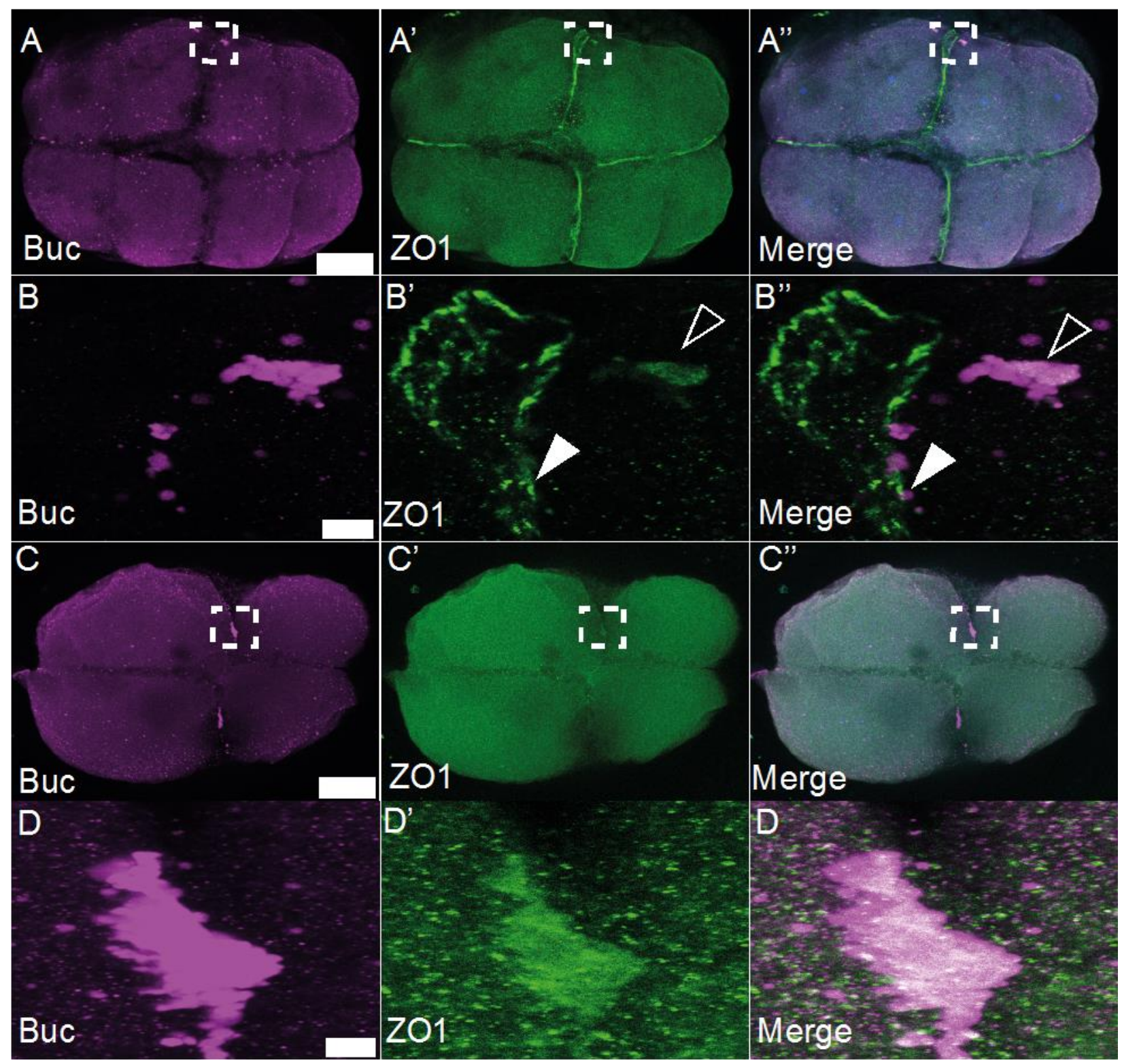

Figure 8: Tight junction protein ZO1 co-localizes with Buc during embryogenesis. Co-localization of Buc with different cellular structure markers during embryogenesis was determined by immunohistochemistry: $1^{\text {st }}$ column - Buc (magenta), $2^{\text {nd }}$ column - cellular structure markers (green) and $3^{\text {rd }}$ column - merge (including DAPI). Animal view of embryos at the 8-cell stage is shown in (A) and the 4-cell stage in (C), including schematic representation of the 8-cell embryo (Buc in magenta, cellular structure markers in green). Dotted rectangles represent magnification shown in panel below (B). Buc co-localizes with tight junction marker ZO1 at the most distal ends of the cleavage furrows (A-A', BB', empty white arrowhead). Furthermore, ZO1 localizes along the first 2 cleavage furrows (A', B', filled white arrowhead). (C-D) At the 4-cell stage Buc and ZO-1 co-localize at the furrows. Scalebars: (A, C): $100 \mu \mathrm{m} ;(\mathrm{B}, \mathrm{D}): 10 \mu \mathrm{m}$ 


\section{Adherensjunction}

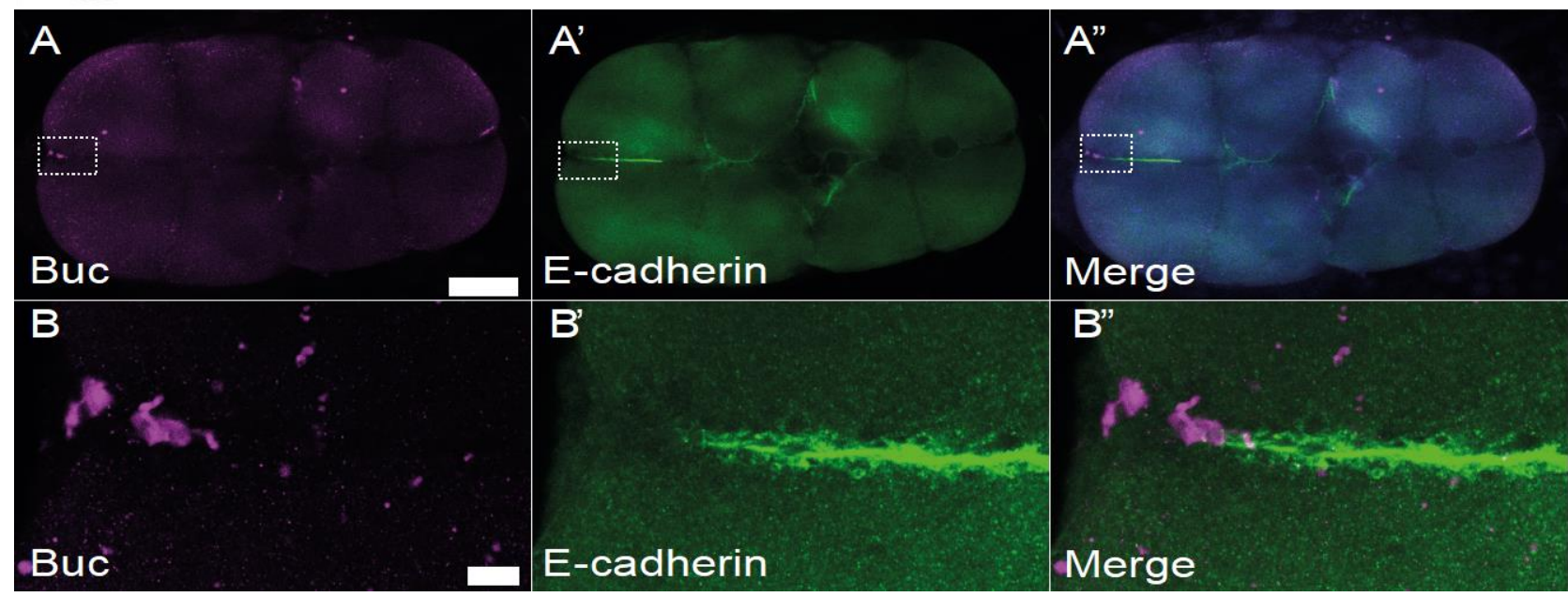

Figure 9: Buc does not co-localize with adherens junctions. Co-localization of Buc with different cellular structure markers during embryogenesis was determined by immunohistochemistry: $1^{\text {st }}$ column - Buc (magenta), $2^{\text {nd }}$ column cellular structure markers (green) and $3^{\text {rd }}$ column - merge (including DAPI). Animal view of embryos at the 8-cell stage is shown in (A), including schematic representation of the 8-cell embryo (Buc in magenta, cellular structure markers in green). Dotted rectangles represent magnification shown in panel below (B). E-cadherin localizes along the first 2 cleavage furrows and does not co-localize with Buc at the distal ends of cleavage furrows (A-A', B-B' '). Scalebars: (A): $100 \mu \mathrm{m} ;(\mathrm{B}): 10 \mu \mathrm{m}$ 


\section{Midbody}

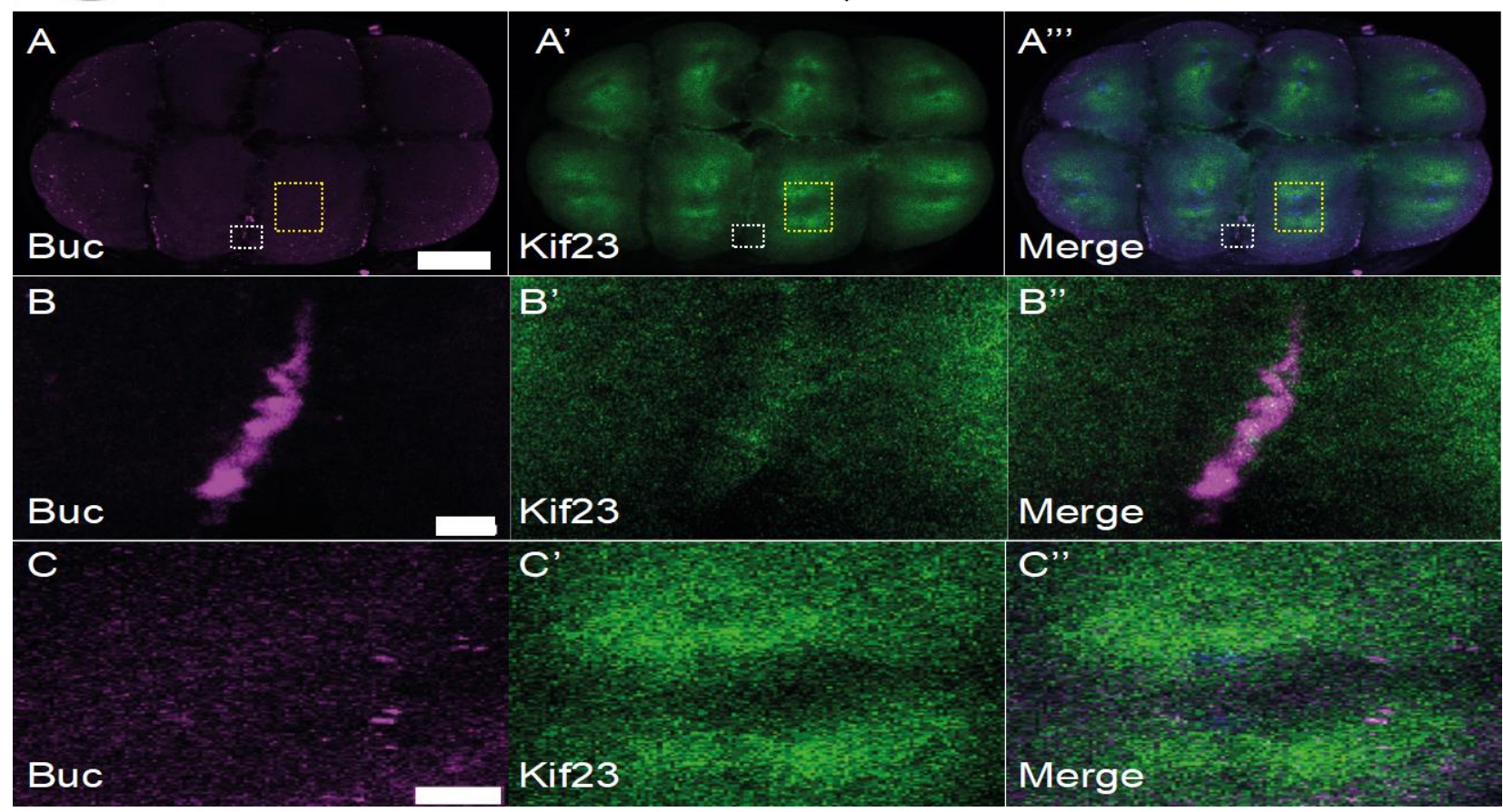

Figure 10: Midbody does not co-localize with Buc during embryogenesis. Co-localization of Buc with different cellular structure markers during embryogenesis was determined by immunohistochemistry: $1^{\text {st }}$ column - Buc (magenta), $2^{\text {nd }}$ column - cellular structure markers (green) and $3^{\text {rd }}$ column - merge (including DAPI). Animal view of embryos at the 8-cell stage is shown in (A), including schematic representation of the 8-cell embryo (Buc in magenta, cellular structure markers in green). Dotted rectangles represent magnification shown in panels below (B, C). Kif23 does not co-localize with Buc (A-A', B-B' '). Instead, Kif23 localizes to cytoplasm (C-C'”) (yellow dotted rectangle magnified in C-C') $)$. Scalebars: (A): $100 \mu \mathrm{m} ;(\mathrm{B}, \mathrm{C}): 10 \mu \mathrm{m}$

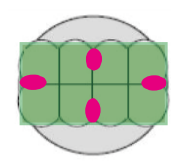

\section{Hemidesmosome}

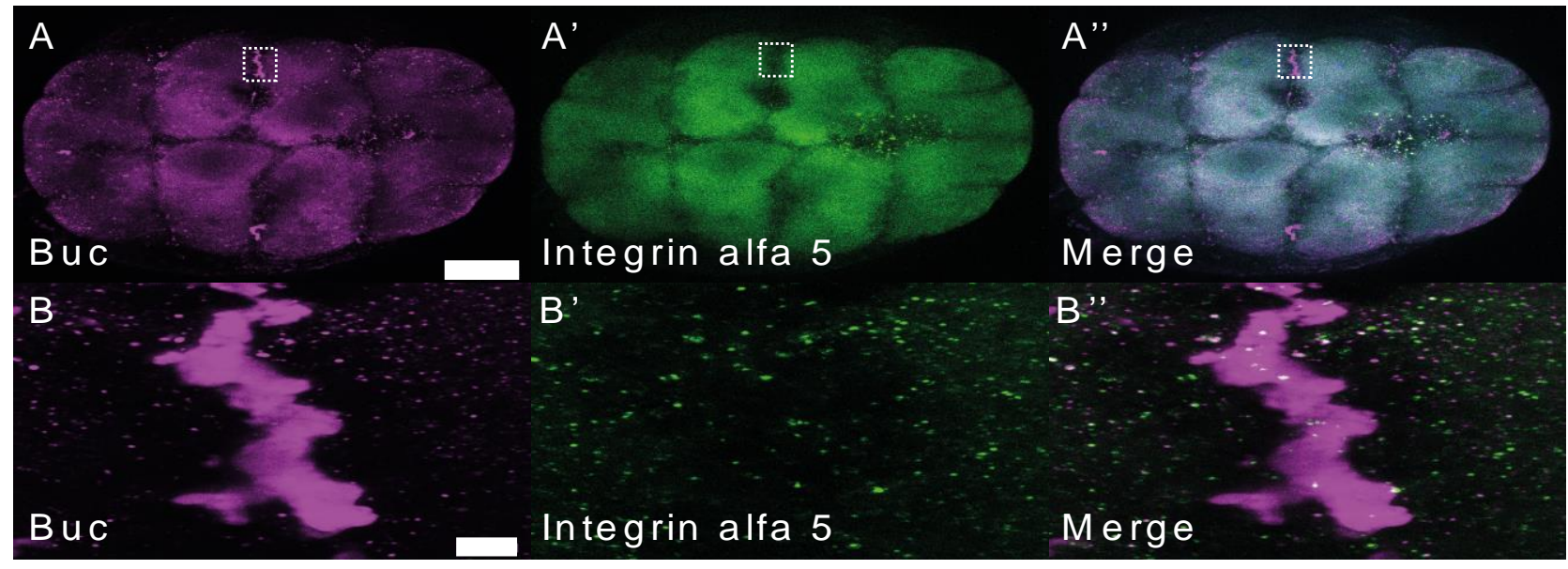

Figure 11: Hemidesmosomes do not co-localize with Buc during embryogenesis. Co-localization of Buc with different cellular structure markers during embryogenesis was determined by immunohistochemistry: $1^{\text {st }}$ column - Buc (magenta), $2^{\text {nd }}$ column - cellular structure markers (green) and $3^{\text {rd }}$ column - merge (including DAPI). Animal view of embryos at the 8-cell stage is shown in (A), including schematic representation of the 8-cell embryo (Buc in magenta, cellular structure markers in green). Dotted rectangles represent magnification shown in panels below (B). Hemidesmosomal marker integrin $\alpha-5$ does not co-localize with Buc, showing ubiquitous cytoplasmic localization (AA', B-B', ). Scalebars: (A): $100 \mu \mathrm{m}$; (B): $10 \mu \mathrm{m}$ 


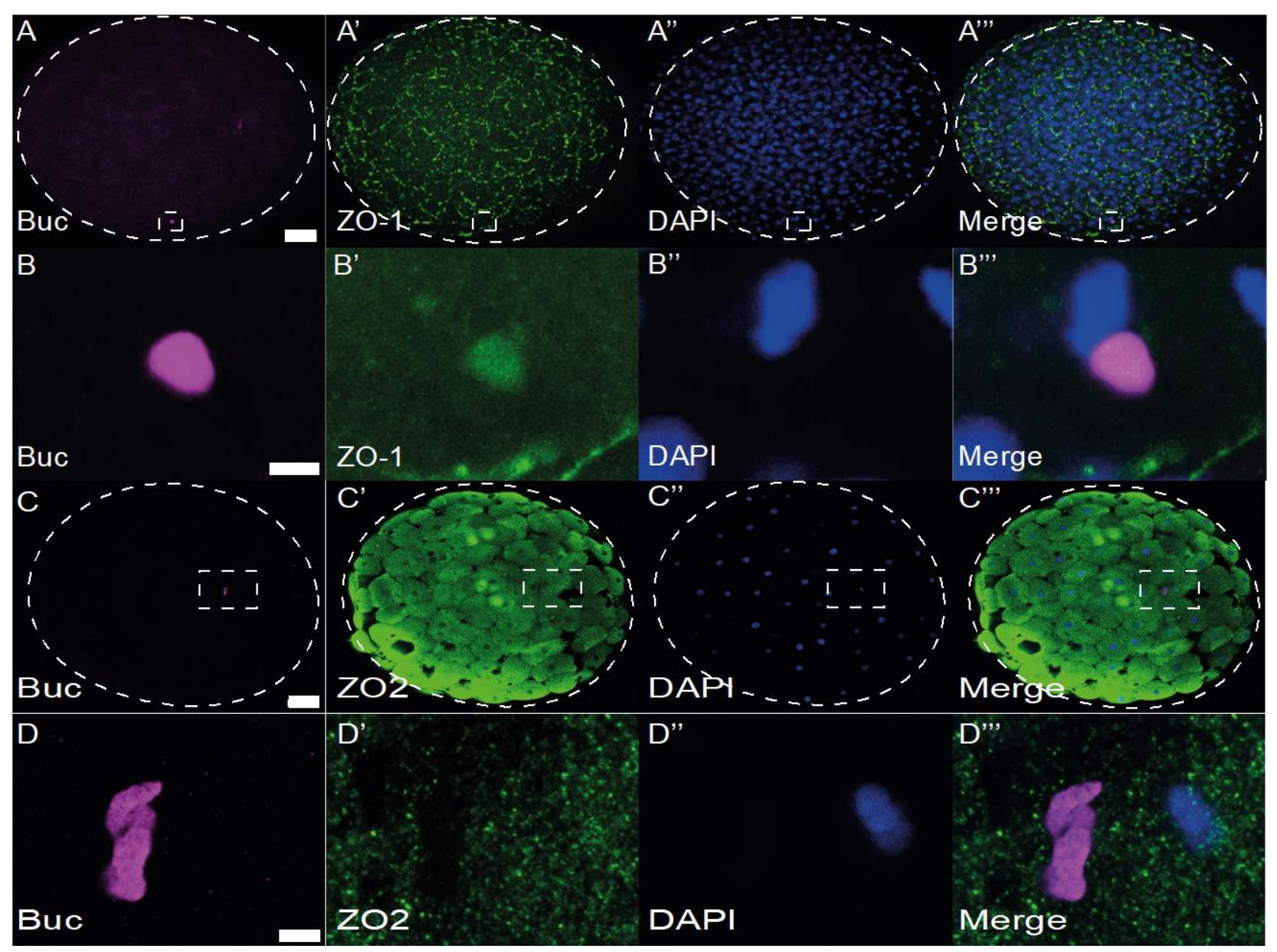

Figure 12: Tight junction protein ZO1 co-localizes with Buc during embryogenesis. Co-localization of Buc with tight junction markers was determined by immunohistochemistry: $1^{\text {st }}$ column - Buc (magenta), $2^{\text {nd }}$ column - cellular structure markers (green) and $3^{\text {rd }}$ column -nucleus (DAPI - blue) and $4^{\text {th }}$ column - merge. Animal view of embryos at the 512-cell stage (A, C). Dotted rectangles represent magnification shown in panel below. Buc co-localizes with tight junction markers ZO1 (A-B), whereas ZO2 does not co-localize with Buc (C-D). Scalebars: (A, C): $50 \mu \mathrm{m} ;(\mathrm{B}, \mathrm{D}): 2 \mu \mathrm{m}$ 


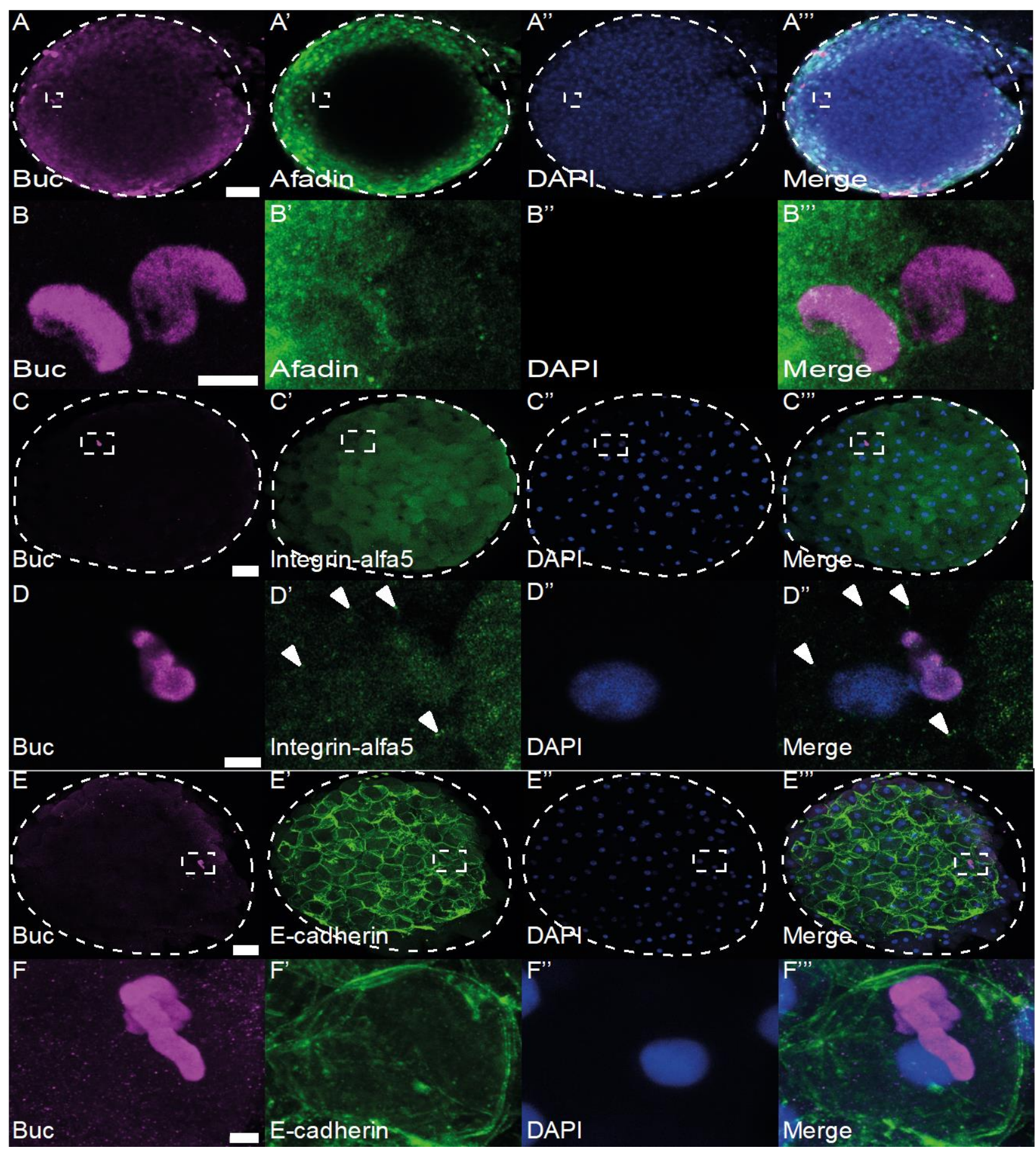

Figure 13: Adherens junctions and hemidesmosomal markers do not co-localize with Buc. Co-localization of Buc with tight junction markers was determined by immunohistochemistry: $1^{\text {st }}$ column - Buc (magenta), $2^{\text {nd }}$ column - cellular structure markers (green) and $3^{\text {rd }}$ column -nucleus (DAPI - blue) and $4^{\text {th }}$ column - merge. Animal view of embryos at the 512-cell stage is shown. Dotted rectangles represent magnification shown in panel below. Buc does not co-localize with adherens junction markers afadin (A-B) which shows cytoplasmic localization; Buc also does not co-localize with E-cadherin (E-F), which localizes at cell-cell junctions. Furthermore, Buc and Intergin alfa 5 do not co-localize (C, D). Integrin alfa 5 localizes in single points (D, D', ', white arrowheads). Scalebars: (A, C, E): $50 \mu \mathrm{m} ;(\mathrm{B}): 2 \mu \mathrm{m} ;(\mathrm{D}, \mathrm{F}): 5$ $\mu \mathrm{m}$ 


\subsubsection{Early embryo contains germ plasm specific and somatic Z01}

Previously we found that Buc co-localizes with ZO1 at the distal ends of the first two cleavage furrows at the 8-cell stage (Figure 8). We also found the majority of ZO1 localized along the entire length of the first two cleavage furrows in the same immunostaining. These results suggested the presence of at least two different $\mathrm{ZO} 1$ isoforms: one is responsible for germ plasm localization and one - for establishment of somatic tight junctions.

In order to investigate presence of different $\mathrm{ZO} 1$ isoforms in early embryos, we performed immunostaining with a different ZO1 antibody (anti-rat). We dubbed the detected ZO1 by this antibody as ZO1-R and the previously detected ZO1 (anti-mouse) as ZO1-M (Figure 8). Buc and ZO1-R co-localized at the distal ends of the first two cleavage furrows at the 8-cell stage (Figure 14 $\underline{\mathrm{D}, \mathrm{E})}$. Also, we found ZO1-R in Buc granules at the 512-cell stage and in cytoplasm (Figure $14 \mathrm{~F}$, G). In contrast, ZO-1-M co-localized with Buc and to the entire length of the first two cleavage furrows at the 8-cell stage (Figure $14 \mathrm{~A}, \mathrm{~B}$ ) and to all cleavage furrows at the 16-cell stage (Figure $14 \mathrm{~A}, \mathrm{~B})$. Thus, ZO1-R probably represents a germ line specific ZO1 isoform, while ZO1-M - a somatic isoform. Recently, we obtained the same result for immunostaining of ZO1 with antibodies against ZO1-A isoform as with the anti-rat ZO1 antibody (Rostam, unpublished). Thus, we assume that ZO1-A co-localized with germ plasm. Hence, ZO1-A might be responsible for anchoring germ plasm to cytoskeleton.

In summary, we show for the first time the presence of two different maternal ZO1 isoforms. We assume that the germline specific ZO1-A defines which cells will become germ cells by anchoring and stabilizing germ plasm early during development. In contrast, the somatic ZO1 might function as part of regular tight junctions. 


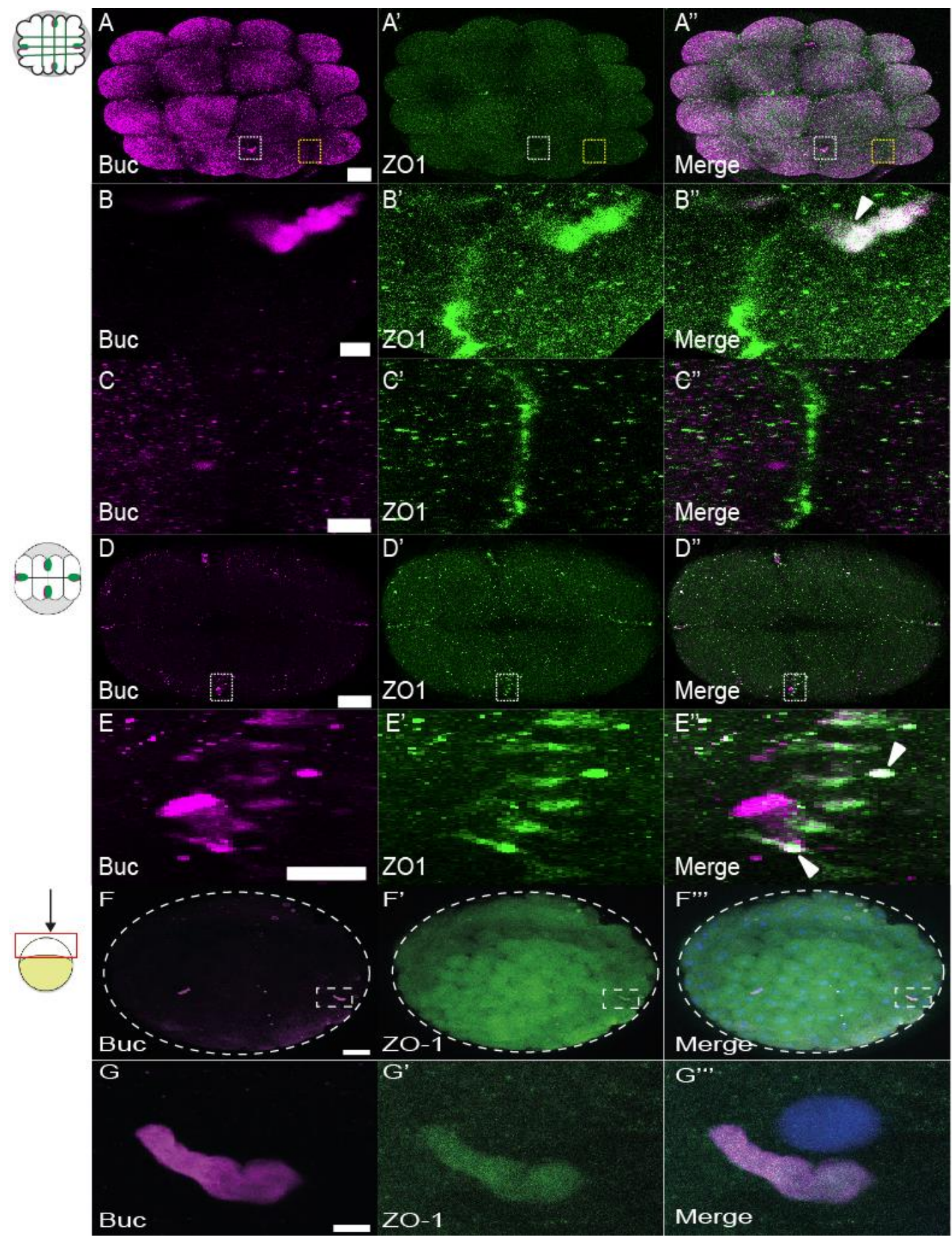

Figure 14: Early embryo contains germline specific and somatic ZO1. Immunostaining of embryos for ZO1 was performed with two different antibodies. (A-C) detected ZO1 with anti-mouse antibody (dubbed as ZO1-M); (D-G) detected ZO1 with anti-rat antibody (dubbed as ZO1-R). Animal view of the 16-cell stage (A), the 8-cell stage (D) and the 512-cell stage (F) embryos. Dotted rectangles represent magnification shown in panel below. (A) white rectangle germ cell specific ZO1 (magnified in B) and yellow rectangle - somatic ZO1 magnified in (C). Co-localization of ZO1 with germ plasm was determined by immunohistochemistry: $1^{\text {st }}$ column - Buc (magenta), $2^{\text {nd }}$ column - cellular structure markers (green) and $3^{\text {th }}$ column - merge (including DAPI only for F-G). (A-C) ZO1-M co-localizes with Buc at the distal ends of the first two cleavage furrows and is also present along entire length of all cleavage furrows at the 16-cell stage. (D-E) ZO1-R co-localizes with Buc at the distal ends of the first two cleavage furrows and is not localized along the entire length of cleavage furrows at the 8-cell stage. (F-G) Buc and ZO1-R co-localize at the 512-cell stage. Scalebars: (A, D, F): $50 \mu \mathrm{m}$; (B, C, E, G): $5 \mu \mathrm{m}$; (D, E performed by Rostam). 


\subsubsection{Phosphorylation of $\mathrm{ZO1}$ is required for degradation of Buc}

We demonstrated that Buc and ZO1 co-localize during early embryogenesis (Figure 8). To address the function of ZO1 in germ plasm, we used a specific inhibitor of protein kinase $\mathrm{C}$ (PKC) Calphostin C (CC) (Stuart and Nigam, 1995). CC reduces phosphorylation of ZO1 and inhibits tight junction biogenesis in-vitro (Stuart and Nigam, 1995). We expected that ZO1 dephosphorylation has a negative effect on Buc localization. For this, we treated embryos containing Buc-GFP transgene with CC, keeping drug concentration the same as it was described for treatment of the cell cultures $500 \mathrm{nM}$ (Stuart and Nigam, 1995). We treated embryos at the 1-cell stage and scored embryos for presence of localized or unlocalized Buc-GFP at 3-4 hpf by live-imaging. We found elevated levels of unlocalized Buc-GFP in CC treated embryos (Figure $15 \mathrm{~A}-\mathrm{C}, \mathrm{F}$ ). Besides, we found the number of Buc-GFP granules was the same in both $\mathrm{CC}$ treated or untreated condition (Figure $15 \mathrm{E}$ ), while the intensity of localized Buc-GFP was higher in CC treated embryos (Figure 15 B). Remarkably, upon a higher $\mathrm{CC}$ concentration $(5 \mu \mathrm{M})$, we found a much higher increase in background GFP fluorescence in buc-gfp embryos already at the 16-cell stage (Figure 16). Furthermore, to demonstrate the effect of the PKC inhibitor on Buc-GFP levels, we performed a western blot with CC treated and untreated embryonic lysates. We also found elevated Buc-GFP protein in CC treated embryos (Figure $15 \mathrm{D})$. Thus, we concluded that altering ZO1 phosphorylation status causes a decline of Buc degradation.

Previously it was demonstrated that zebrafish germ plasm granules significantly decrease in size during the first 24 hours of development (Strasser et al., 2008). This implies on presence of a mechanism that removes excess germ plasm as an embryo develops (Strasser et al., 2008). Also, we observed that unlocalized Buc is degraded between 1 and 32-cell stages, while the localized Buc granules also reduce in size during the first hours of embryonic development (Riemer et al., 2015). Hence, we hypothesized that levels of Buc should increase already at the 8-cell stage upon CC treatment. To address this, we performed immunohistochemistry to visualize Buc and ZO1 at the 8cell stage. Treatment of embryos with $500 \mathrm{nM} \mathrm{CC}$ did not result in any increase of localized or unlocalized fraction of Buc (data not shown). In contrast, treatment of embryos with $3 \mu \mathrm{M} \mathrm{CC}$ resulted in elevated levels both Buc fractions (Figure 17). Firstly, the elevated levels of Buc are observed at the cortex, where unlocalized Buc forms granules (Figure $17 \mathrm{~A}, \mathrm{~B}$ ). Secondly, higher levels of Buc are found at all cleavage furrows. Thus the elevated Buc-GFP levels observed in the live-imaged embryos (Figure $15 \mathrm{~A}-\mathrm{C}$ ) and the western blot with embryo lysates (Figure $15 \mathrm{D})$ are probably a consequence of an early decline of Buc degradation upon $\mathrm{CC}$ treatment.

Upon $3 \mu \mathrm{M}$ CC treatment, embryos also displayed increased ZO1 levels at the cleavage furrows (Figure 17). It seems like CC induced accumulation of cytoplasmic ZO1 pool to the cleavage furrows. This is in contrast to Buc, which still shows high levels of cytoplasmic accumulation in CC treated embryos (Figure 17).

Taken all results together, we conclude that $\mathrm{ZO} 1$ phosphorylation is required for degradation of the unlocalized and some part of localized Buc during early embryogenesis. 

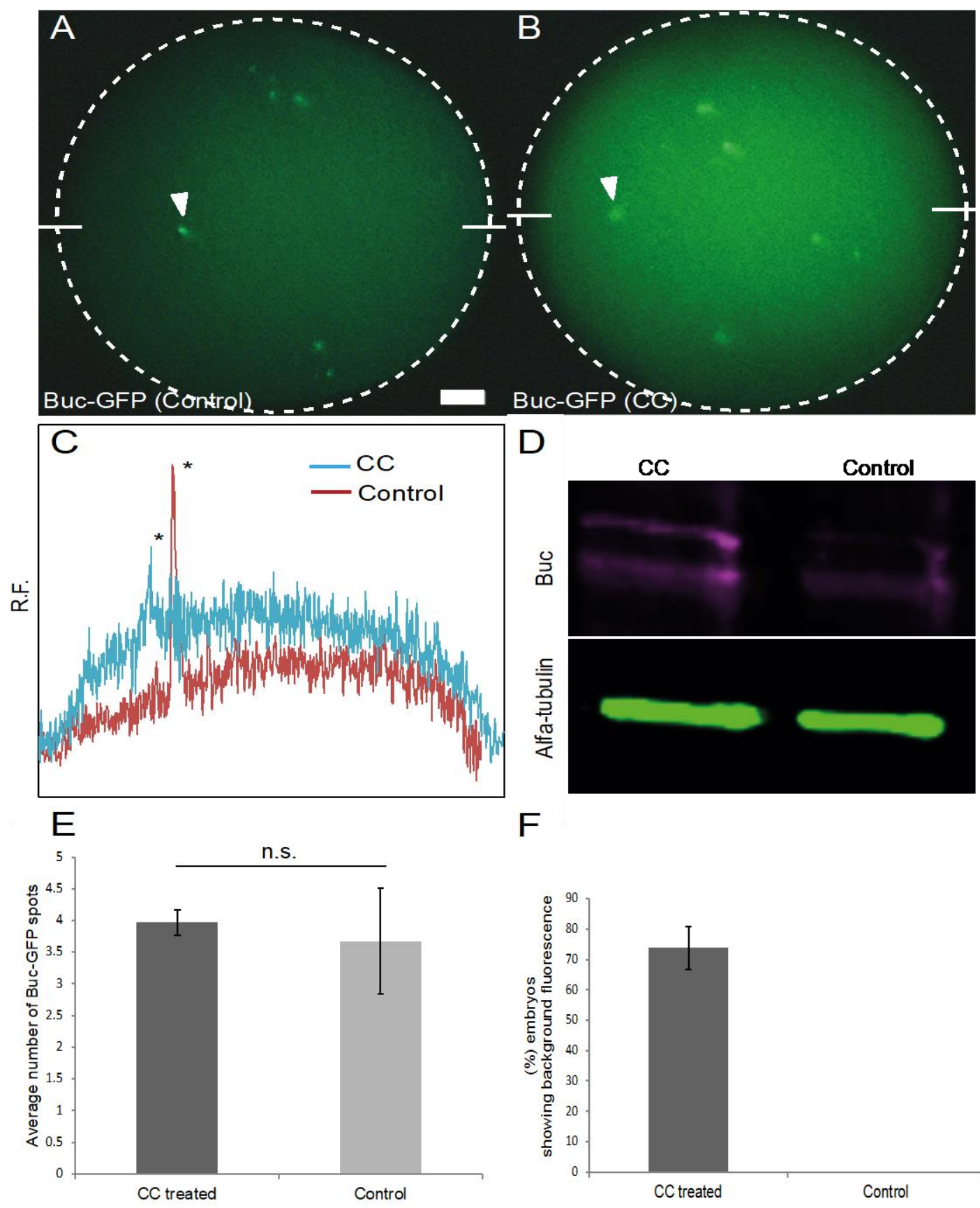

F

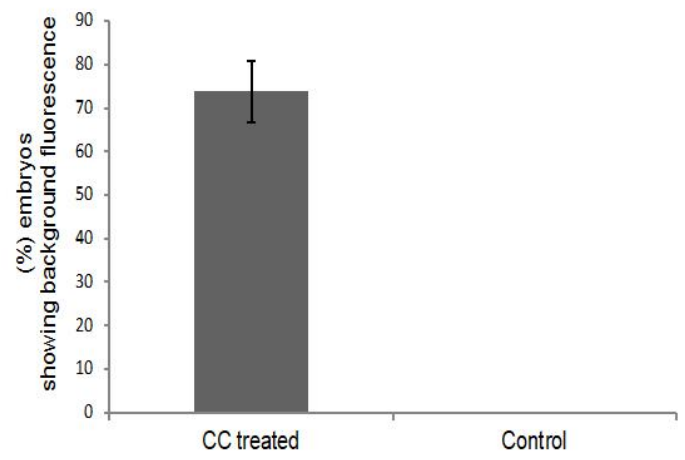

Figure 15: ZO1 phosphorylation is required for Buc degradation. The role of $\mathrm{ZO} 1$ in Buc localization was investigated using a ZO1 specific phosphorylation inhibitor Calphostin C (CC). (A-B) Animal view of living 3 hours post fertilization embryos transgenic for Buc-GFP. Embryos were treated with DMSO (control) or CC at the 1-cell stage. Dotted circle outlines the embryo. The plot in (C) shows levels of fluorescent intensity along the line indicated by white dashes in (A-B). Asterisks in (C) indicate Buc-GFP spots highlighted by white arrowheads in (A-B). CC treated embryos show higher background fluorescence compared to the control embryos. (D) Western blot with lysates of CC treated and control embryos. Upper blot shows endogenous Buc (magenta), whereas the lower blot shows loading control alfa-tubulin (green). CC treated embryos show higher levels of endogenous Buc compared to control embryos. (E) Quantification of transgenic Buc-GFP spots in (A-B). How is done. No significant difference was found in number of Buc-GFP spots between $\mathrm{CC}$ treated (4.0 spots \pm 0.2 ) and control embryos (3.7 spots \pm 0.8$)$. (F) Quantification of embryos showing background GFP fluorescence in (A-B). The percentage of $\mathrm{CC}$ treated embryos showing background fluorescence (73.8 $\pm 7.1 \%)$ is significantly higher than among the control embryos (0\%). Scalebars: (A-B): $50 \mu \mathrm{m}$ 

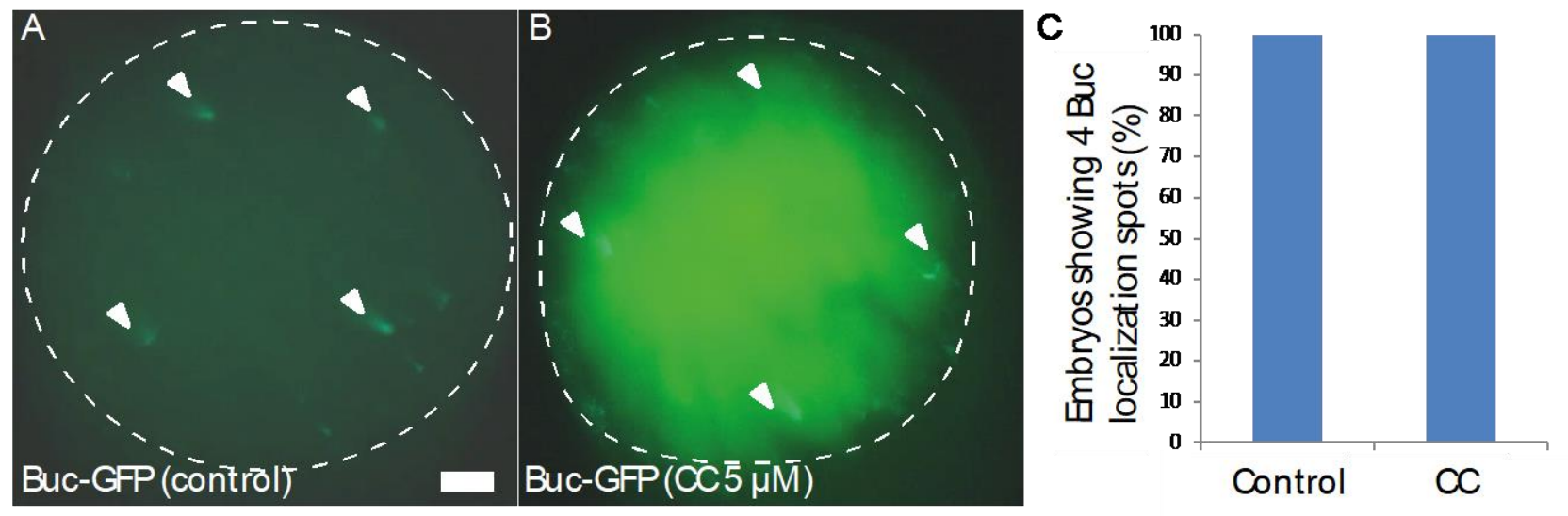

Figure 16: ZO1 phosphorylation is required for Buc degradation. The role of $\mathrm{ZO} 1$ in Buc localization was investigated using a ZO1 specific phosphorylation inhibitor Calphostin C (CC). (A-B) Animal view of living 16-cell stage buc-gfp line embryos. Embryos were treated with DMSO (control) or CC $(5 \mu \mathrm{M})$ at the 1-cell stage and scored for presence of GFP granules at the 16-cell stage. Dotted circle outlines the embryo. Arrowheads in (A, B) indicate Buc-GFP spots. (A, B) CC treated embryos show higher background fluorescence compared to the control embryos. (C) Quantification of transgenic Buc-GFP spots in (A-B) shows no difference in number of GFP granules $(\mathrm{n}=5, \mathrm{~N}=1)$. Scalebars: (A, B): $50 \mu \mathrm{m}$ 


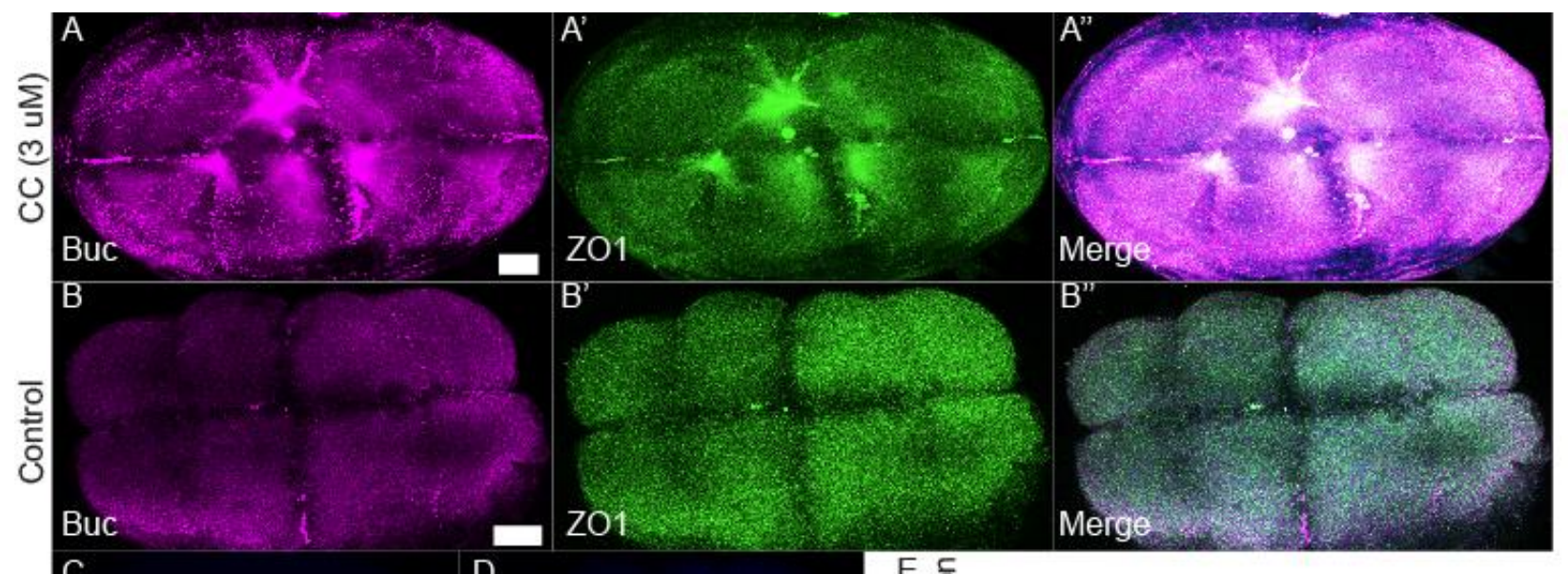

C

$\mathrm{D}$

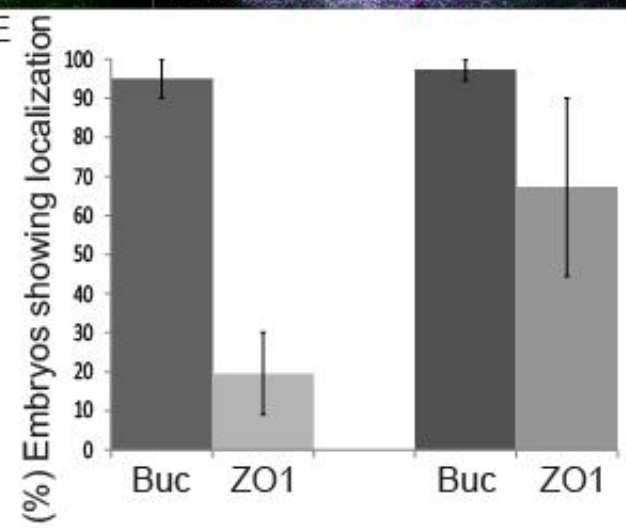

Figure 17: ZO1 phosphorylation is required for Buc degradation. The role of $\mathrm{ZO} 1$ in Buc localization was investigated using a ZO1 specific phosphorylation inhibitor Calphostin C (CC). Embryos were treated with $3 \mathrm{uM} \mathrm{CC}$ (A), including untreated control (B). Localization of Buc and ZO1 was determined by immunohistochemistry (A-B): $1^{\text {st }}$ column: endogenous Buc (magenta), $2^{\text {nd }}$ column - endogenous ZO1 (green) and $3^{\text {rd }}$ column - merge. Nucleus was visualized by DAPI (C-D). Animal view of 8-cell stage embryos is shown. (A) Treatment of embryos with $3 \mu \mathrm{M}$ CC results in more enrichment of Buc and $\mathrm{ZO} 1$ at cleavage furrows compared to control (B). (E) shows result from scoring embryos from (A) and (B) for Buc and ZO1 localization. There is no difference in Buc localization between CC treated $(95 \pm 5 \%)$ and untreated embryos $(97.2 \pm 2.8 \%)$, whereas $\mathrm{ZO} 1$ is found less frequently localized in untreated $(19.5 \pm 10.5 \%)$ vs CC treated embryos $(67.2 \pm 22.8 \%)(\mathrm{N}=2, \mathrm{n}=10)$. Scalebars: $100 \mu \mathrm{m}$ 


\subsection{Bucky ball plays a role in germ plasm aggregation and localization}

We showed that germ plasm co-localizes with tight junction proteins, which might link germ plasm to cytoskeleton. However, the identification of PrDs in the N-terminal domain of Buc challenged our view on how germ plasm localization might work (Boke et al., 2016). Also, previous assumption that Buc is an IDP (Jeske et al., 2015) raises a question whether PrDs within the Buc IDRs are required for germ plasm localization. Therefore, it is important to distinguish between aggregation and localization behavior of Buc during early embryogenesis.

\subsubsection{Buc is an intrinsically disorder protein and aggregates in-vitro}

Presence of IDRs in Buc is likely to explain the dynamic liquid droplet behavior of germ plasm during zebrafish embryogenesis (Riemer et al., 2015). However, we did not expect that IDRs contribute to germ plasm localization. Therefore, we first investigated Buc for presence of IDRs. Additionally, we investigate germ plasm organizer Drosophila short Oskar (sOsk) for the presence of IDRs. To investigate Buc and sOsk for presence of IDRs we used the PONDR-VSL2 algorithm (Peng et al., 2006). PONDR-VSL2 is a metapredictor, which conservatively combines the results of prediction algorithms. Interestingly, we found that the first 100 residues containing the BUVE motif of Buc have an ordered structure (Figure $18 \mathrm{~A})$. This result indicates that germ plasm localization is independent of IDRs. We also found that both Buc and sOsk contain large IDRs (Figure $18 \mathrm{~A}, \mathrm{~B}$ ). As a positive control for IDP prediction, we used Vasa which contains the known IDR of about 200 residues at its N-terminus (Nott et al., 2015; Yoon et al., 1997), whereas Ziwi was largely unstructured (Figure 18 $\underline{\mathrm{C}, \mathrm{D})}$. Unlike Buc or Velo1, sOsk does not contain any predicted PrDs. However, it is demonstrated that sOsk aggregates in S2-cells, supporting its prediction as an IDP (Boke et al., 2016). To check if Buc and sOsk form similar aggregates, we transfected HEK293 cells with GFP fusions of both proteins. We found that GFP fusions of Buc and sOsk form aggregates (Figure 18 E, F), whereas GFP alone shows uniform distribution in cells (Figure $18 \mathrm{G})$. When we transfected Buc lacking the most of the predicted ordered region (DeltaBuc aa 11-88) we still observed formation of GFP granules (Figure 21 D-D'). Therefore, similarly to sOsk, Buc can form aggregates independently of the presence of PrDs. Furthermore, upon Buc-GFP and sOsk-GFP co-transfection we found protein aggregates containing both germ plasm organizers (Figure $18 \mathrm{H}-\mathrm{J}$ ). This result indicates that IDRs of Buc and sOsk form aggregates with similar biophysical properties. Thus, the presence of IDRs in Buc accounts for its aggregation behavior.

\subsubsection{Dynamic nature of Bucky ball aggregation in vivo}

Previously, it was shown that Buc and Velo1 form stable aggregates during Xenopus oogenesis (Boke et al., 2016). In contrast to oogenesis, during zebrafish embryogenesis the transgenic Buc-GFP behaves like liquid droplets (Riemer et al., 2015). Therefore, we hypothesized that Buc forms less stable aggregates during embryogenesis than during oogenesis. To address the solubility of Buc aggregates, we used the aliphatic drug 1,6-hexanediol (1,6-HD). 1,6-HD dissolves hydrogels like C.elegans $\mathrm{P}$-granules, but not amyloid-like aggregates like the $\mathrm{Bb}$ in Xenopus oocytes (Updike et al., 2011; Kroschwald et al., 2015; Boke et al., 2016). We found that treatment of transgenic Buc-GFP ovaries with 1,6-HD does not dissolve $\mathrm{Bb}$ (Figure $19 \mathrm{~A}, \mathrm{~B})$. When we doubled 1,6-HD concentration, the $\mathrm{Bb}$ still was not dissolved (Figure $20 \mathrm{~A}-\mathrm{D})$. Nevertheless, HD treatment depleted a fraction of 
Buc-GFP granules from the $\mathrm{Bb}$ (Figure $19 \mathrm{~B}-\mathrm{B}$ ', Figure $20 \mathrm{~B}, \mathrm{D}$ ). However, the most of Buc-GFP remained stable in the $\mathrm{Bb}$ upon 1,6-HD treatment. This result indicates that majority of Buc protein in oocytes forms a stable matrix in $\mathrm{Bb}$, as it was shown for Velo1 during Xenopus oogenesis (Boke et al., 2016). Furthermore, we find that there is a fraction of Buc-GFP which forms less stable aggregates in $\mathrm{Bb}$ and therefore is more prone to 1,6-HD wash out.

In contrast to the 1,6-HD treatment of transgenic Buc-GFP oocytes, we found that 1,6-HD treatment of embryos results in germ plasm fragmentation (Figure $19 \mathrm{D}-\mathrm{F}, \mathrm{I}-\mathrm{J}$ ). The germ plasm fragmentation is observed $30 \mathrm{~min}$ after 1,6-HD treatment (Figure 19 D-F) and remained evident at 3hpf (Figure 19 $\underline{\mathrm{I}-\mathrm{J})}$. In contrast to 1,6-HD treatment of oocytes, after HD washout in embryos some germ plasm granules were no longer localized to cleavage furrows (Figure $19 \mathrm{G}-\mathrm{H})$. These results can explain an increased number of Buc-GFP granules found in $3 \mathrm{hpf}$ embryos (Figure $19 \mathrm{D}-\mathrm{F})$. We furthermore demonstrated that 1,6-HD does not disrupt cytoskeleton in embryos (Figure $20 \mathrm{M}-\mathrm{T}$ ). Therefore, the observed germ plasm fragmentation is dependent solely on disruption of hydrogels. However, the embryonic germ plasm never completely dissolved like shown for the C. elegans ovarian P-granules (Figure $19 \mathrm{D}-\mathrm{F}$ ). As a control for 1,6-HD specificity, we used a similar chemical 1,2,3-hexanetriol (HT). We found that a more polar structure of HT disrupted Buc-GFP aggregates less efficiently than 1,6-HD (Figure 19 I-J). We concluded that zebrafish germ plasm forms an intracellular hydrogel during embryogenesis. 

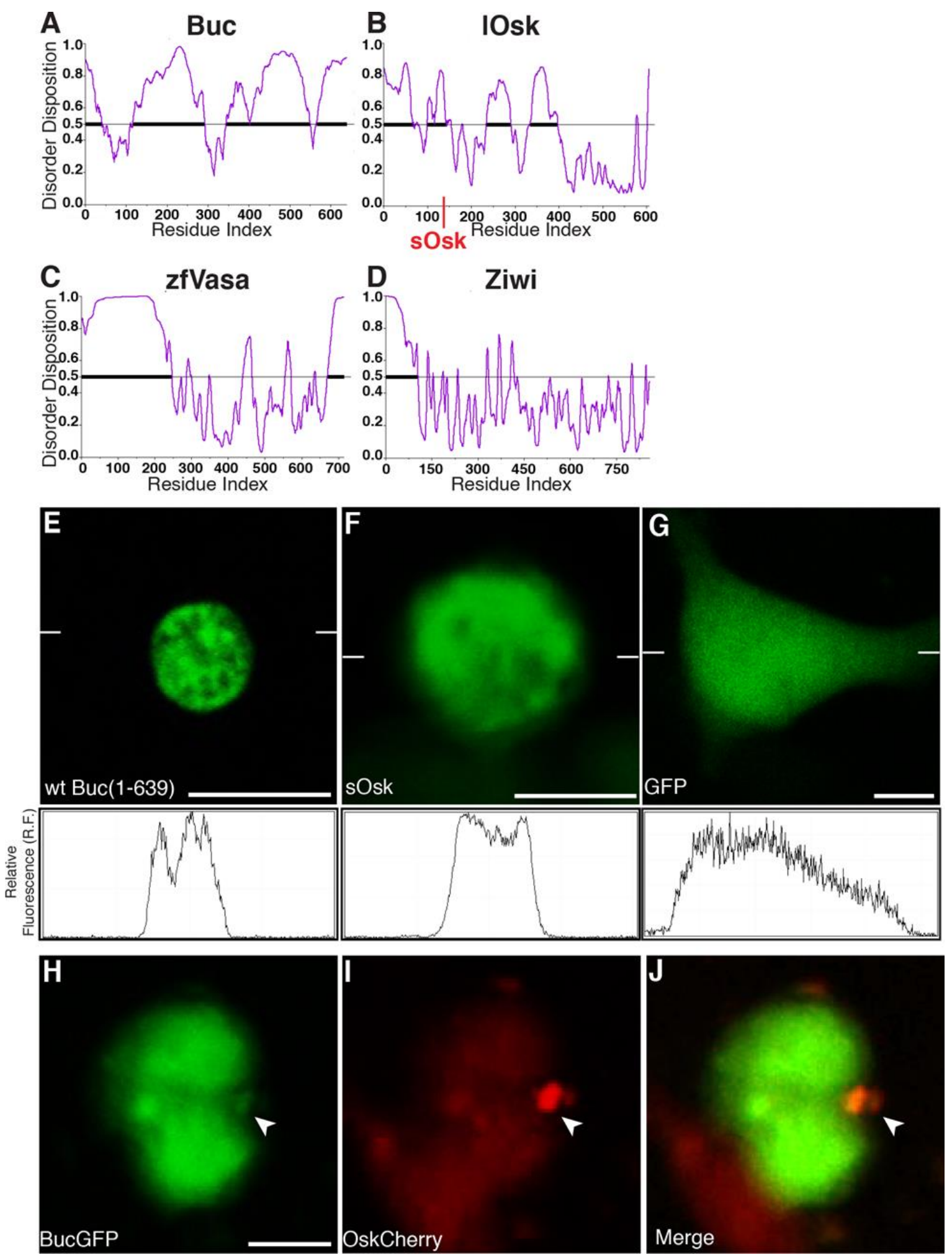

Figure 18: Buc and Osk contain intrinsically disordered regions and aggregate in HEK cells. Predicted protein disorder in (A) Buc, (B) Osk, (C), Vasa, and (D) Ziwi. Disorder disposition (y-axis) plotted against the amino acid residue index (x-axis). Values above the 0.5 threshold (grey bar) show the propensity for disordered regions (bold line). The red line at aa 139 in Osk indicates the alternative translation initiation site for short Oskar. Protein aggregates upon transfection of HEK cells with monomeric GFP (mGFP) fused to (E) Buc, (F) sOsk or (G) unfused. The profiles below the pictures show levels of fluorescent intensity along the line indicated by white dashes. Buc-mGFP (green; H) and OskCherry aggregates (red, I) overlap (J, yellow, white arrowhead). Scale bar (E-J): $10 \mu \mathrm{m}$. (A-D: done by P. Krishnakumar) (Figure is taken from Krishnakumar et al., 2018) 

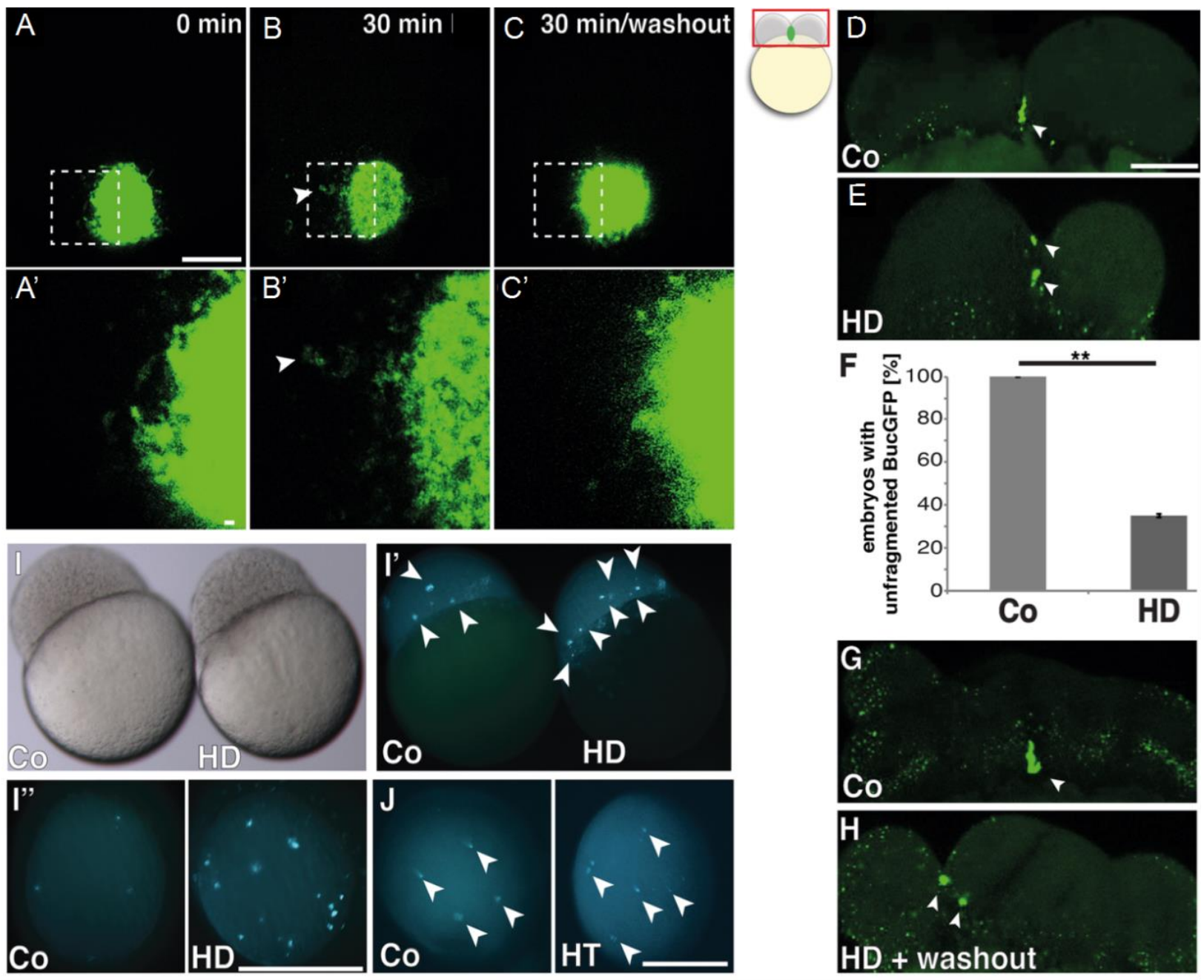

Figure 19: Dynamic nature of Bucky ball aggregation in vivo. (A-C) Balbiani body of living Buc-GFP transgenic oocytes, either before (A), after a 30 min treatment with 5\% 1,6-hexanediol (HD) (B), or 30 min after washout of the drug (lateral view, animal to the top). Arrowheads in B and B' indicate Buc-GFP granule outside the Balbiani body. Scale bar (A-C): $20 \mu \mathrm{m}$ (A'-C'): $2 \mu \mathrm{m}$. (D-K) Germ plasm of transgenic Buc-GFP embryos after hexanediol treatment (HD). (D, E) lateral view of living 2-cell embryo as shown in boxed area of icon. Control embryos show unfragmented Buc-GFP aggregates (green) (D arrowhead), whereas 5\% hexanediol for 30 min leads to fragmentation(arrowheads). (F) Quantification of embryos with unfragmented Buc-GFP in control $(\mathrm{Co} ; 100 \pm 0 \% ; \mathrm{n}=20)$ and embryos treated for 30 min with hexanediol (HD; 35.0 $\pm 0.8 \% ; \mathrm{n}=20 ; \mathrm{p}=0.0065)$. Student's t-test; P-value: $* *<0.01$. (G, H) lateral view of living 4cell embryos. Control embryo with unfragmented BucGFP (green, arrowhead), whereas Buc-GFP stays fragmented 30 min after washout of hexanediol (green; arrowheads). Scale bar (D-H): $100 \mu \mathrm{m}$. (I-K) Buc-GFP aggregates in 3 hpf embryos transgenic for Buc-eGFP. (I) The morphology of control (Co) and hexanediol-treated embryos (HD). Lateral view, animal to the top. (I', I") Fragmented Buc-GFP aggregates (white arrowheads) persist until 3 hpf (I') lateral view, (I") animal view. (J) Treatment with hexanetriol (HT) also leads to fragmented germ plasm (right embryo in J; animal view). Scale bar (I-J): $500 \mu \mathrm{m}$. (I-D: done by P.Krishnakumar) (Figure is taken from Krishnakumar et al., 2018) 


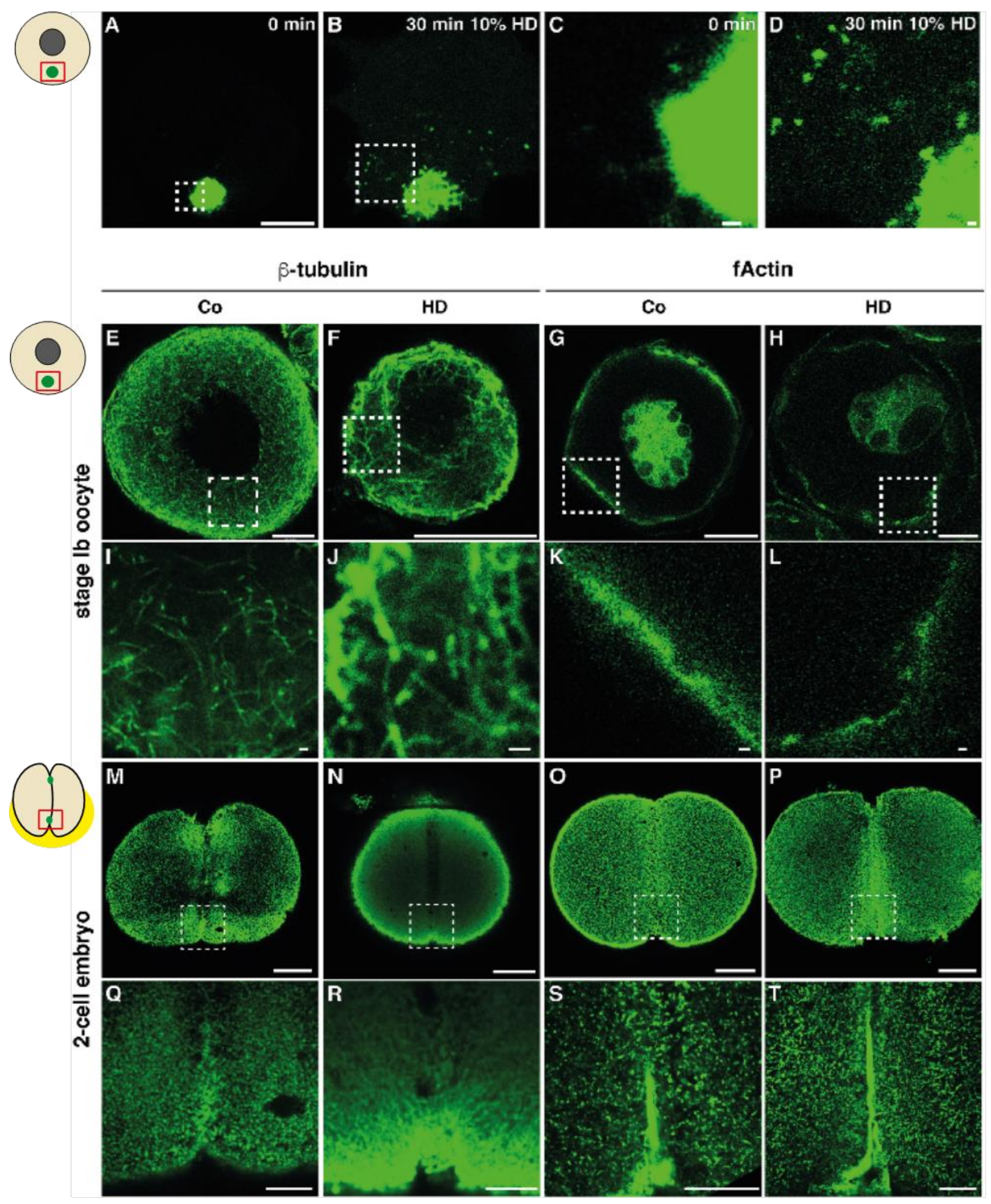

Figure 20: Dynamic nature of Bucky ball aggregation in vivo. Buc-GFP (green) in the Balbiani body of stage $\mathrm{Ib}$ oocytes before hexanediol treatment (A, C; 0 min) or after 30 min treatment with double conc. (10\%; B, D). Stippled squares indicate the magnified area shown in panel $\mathrm{C}$ and $\mathrm{D}$. Note the BucGFP fragments draining off the Balbiani body after HD treatment (D). Scale bar (A, B): $20 \mu \mathrm{m}$; (C, D): $1 \mu \mathrm{m}$. Cytoskeleton after Hexanediol treatment. Oocytes (E-L) or embryos (M-T) were treated for $30 \mathrm{~min}$ with hexanediol and stained for microtubules ( $\beta$-tubulin) or microfilaments (filamentous Actin). Stippled boxes (E-H, M-P) indicate magnified area (I-L, Q-T). 2-cell embryos (M-T) are shown in animal view. Scale bars (E-H, Q-T): $20 \mu \mathrm{m}$. (I-L): $1 \mu \mathrm{m}$. (M-P): $100 \mu \mathrm{m}$. (Figure is taken from Krishnakumar et al., 2018) 


\subsubsection{N-terminal amino acids are sufficient for germ plasm localization}

Previously, it was shown that Buc11-88 is necessary and sufficient for germ plasm localization. Furthermore, further mapping of Buc11-88, by continuous splitting this domain in half, did not result in any fluorescent granule formation. However, mapping Buc11-88 in this way did not provide enough resolution on amino acid level to understand if Buc11-88 represents a minimal localization domain. Identifying a minimal localization sequence can simplify searching for protein domains and single amino acids critical for germ plasm localization. Furthermore, a smaller localization motif can be used as bait for a more precise co-immunoprecipitation of Buc interactors. This allows performing a mass spectrometry analysis to find a core interactome of the localization domain.

Studying germ plasm localization in zebrafish is more time laborious than investigating it using an in-vitro cell culture. Therefore, first we exploited HEK293 to study germ plasm localization. We transfected GFP fusions of buc, buc11-88, bucdelta11-88 and gfp alone into HEK293 cells and investigated cell for presence of GFP granules after 48 hours (Figure $21 \mathrm{~A})$. We found that that Buc1188 formed GFP granules (Figure 21 C-C' '), similarly to full length Buc (Figure 21 B-B') . In contrast, GFP alone showed ubiquitous protein distribution (Figure 21 E-E'). Unfortunately, BucDelta11-88 showed fluorescent granule formation in HEK293 cells (Figure 21 D-D'). In contrast, BucDelta1188 has been shown not to form granules in 3-4 hpf embryos (Riemer, 2014, Doctoral dissertation). Therefore, we concluded that in HEK293 cells, Buc11-88GFP granules could also represent protein aggregates. Therefore, the HEK293 cells system is not a suitable system to distinguish germ plasm aggregation and localization.

In order to find the minimal localization signal, we did a refined structure to function analysis of Buc11-88 in zebrafish. In this analysis we cloned and fused buc constructs of different length to $g f p$, injected them into 1-cell stage embryos and scored for presence of GFP granules at 3-4 hpf (Figure $22 \mathrm{~A}$ ). We found that removing 10 C-terminal residues from bucl1-88 did not affect the formation of GFP granules (Figure 22 A, Buc31-88 and Buc31-78), whereas removing $25 \mathrm{~N}$-terminal residues resulted in a significant reduction of the GFP granule formation. Furthermore, removing $15 \mathrm{C}$ terminal residues also significantly reduced the formation granules (Figure 22 A, Buc36-88 and Buc31-71). Hence, we selected the minimal sequence that forms GFP granules to be Buc31-78 (dubbed as BucLoc). 


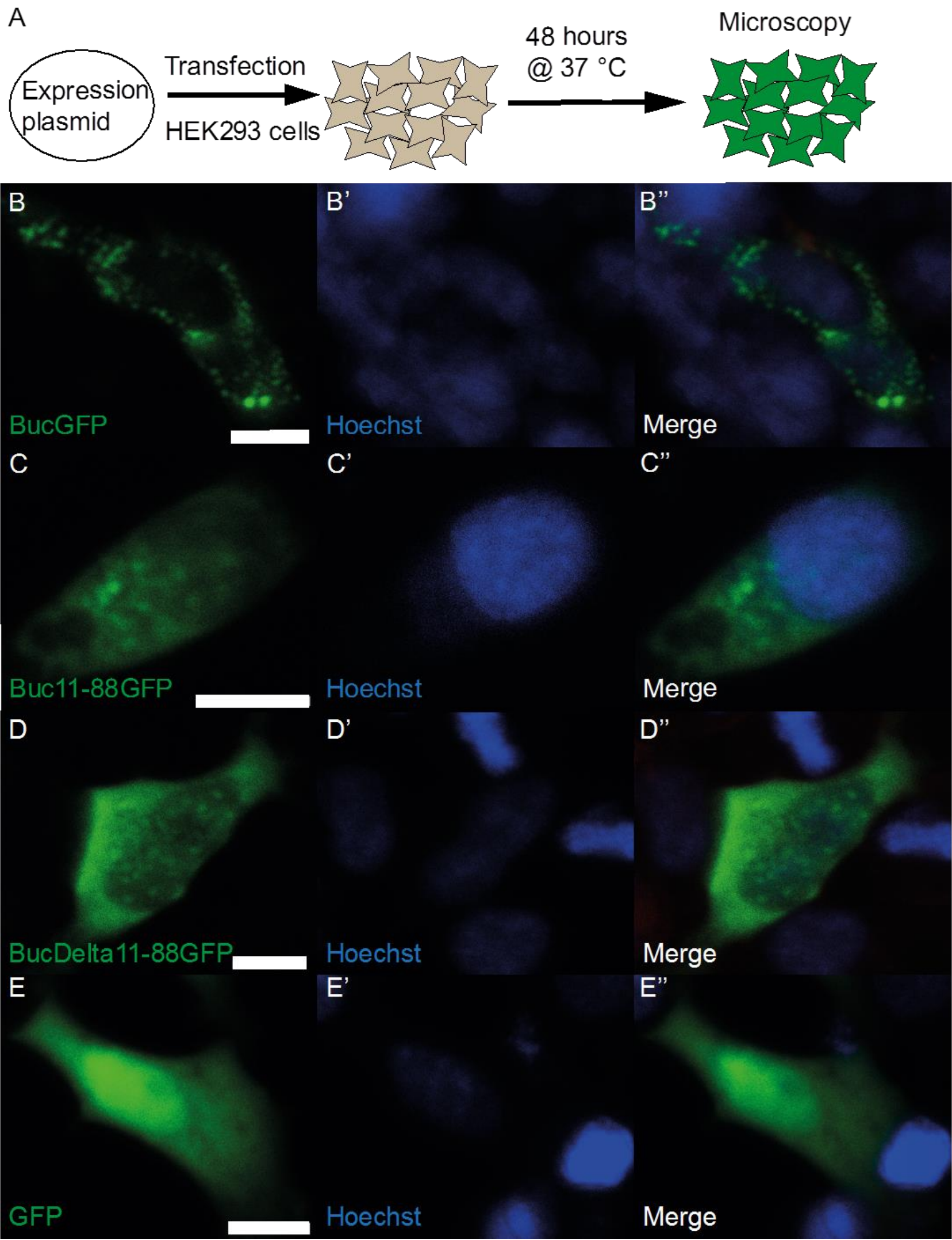

Figure 21: Buc aggregates in HEK293 cells (A) Scheme of HEK293 cells transfection assay. Expression plasmids were transfected at timepoint 0 , incubated for 48 hours at 37 degrees ${ }^{\circ} \mathrm{C}$ and investigated for presence of fluorescent granules by confocal microscopy. (B-E) Live images of single cells showing: $1^{\text {st }}$ column - GFP (green), $2^{\text {nd }}$ column - nucleus (blue) and $3^{\text {rd }}$ column - merge. GFP fusions of Buc (B-B'), Buc11-88 (C-C') or BucDelta11-88 (D-D') form aggregates, whereas GFP alone (E-E') shows ubiquitous fluorescence. Scalebars: $10 \mu \mathrm{m}$. 


\subsubsection{Prion domains are not required for germ plasm localization}

Previously, it was shown that Buc targets to and aggregates in Xenopus Bb. It was hypothesized that this aggregation behavior of Buc is driven via PrDs, located within the BUVE motif (Boke et al., 2016). Buc homolog in Xenopus Velo1 carries two PrDs in its BUVE motif (Boke et al., 2016). These PrDs are necessary for Velo1 aggregation. However, exact locations and sequences of potential PrDs in Buc were not identified, as well as their requirement for Buc aggregation. We showed that Buc forms insoluble structures in zebrafish $\mathrm{Bb}$ (Figure 19 A-C, Figure 20 A-D) (Krishnakumar et al., 2018). Thus, PrDs within the BUVE motif of Buc might be responsible for the Bb aggregation. However, we also found that zebrafish germ plasm is more soluble during embryogenesis, when germ plasm localization becomes important to specify the germline (Figure $19 \mathrm{D}-\mathrm{J}$ ). Therefore, we hypothesized that PrDs do not aggregate during embryogenesis and that germ plasm localization does not depend on aggregation.

Since Velo1 contains two functional PrDs, we first checked whether these two domains are conserved in Buc (Boke et al., 2016). For this we performed a computational analysis of Buc and Velo1 with five web-based aggregation prediction algorithms. We found that Buc contains two prion-like domains which are similar to the prion-like domains in Velo1 (Figure 23 A). Mapping of the Buc1188 domain has already shown that the $\mathrm{N}$-terminal $\mathrm{PrD}$ is not required for germ plasm localization (Figure 22). In order to investigate the role of the C-terminal PrD in germ plasm localization, we made a mutant of this domain BucLoc Y66D, F68D, Y71D (dubbed as BucLoc 3D), similarly as it was done with Velo1 (Boke et al., 2016).

BucLoc 3D mutant was expected to be defective in aggregation, because mutating aromatic residues like phenylalanine or tyrosine into charged residues like aspartic acids results in disruption of protein aggregates (Boke et al., 2016). A computational analysis of Buc 3D with the PLAAC (Prion-Like Amino Acid Composition) web application showed a reduced propensity of BucLoc 3D to behave like a prion-like protein compared to the WT Buc (Figure $23 \mathrm{~B}, \mathrm{C})$. PLAAC scores PrDs in a protein based on its enrichment in asparagine and glutamine residues (Lancaster et al., 2014). This results is similar to what was found for Velo1 4D or 7D mutants in a similar computation analysis (Boke et al., 2016). Then, we investigated the localization of BucLoc 3D mutant to the endogenous germ plasm. For this, we injected RNA of BucLoc mCherry fusion into 1-cell stage embryos of transgenic buc$g f p$ line (Figure $24 \mathrm{~A})$. This approach to study co-localization of a protein with germ plasm we called germ plasm co-localization assay. We found that BucLoc 3D mutant was expressed at low levels, compared to the WT BucLoc (Figure 24 A B, C, D, E). At this low level of expression, BucLoc 3D weakly co-localized with endogenous germ plasm. Hence, we concluded that the $3 \mathrm{D}$ mutation affects the BucLoc expression levels but does not result in a complete loss of localization.

To further investigate the role of the C-terminal PrD in germ plasm localization, we created a mutant BucLoc $\Delta 64-71$, representing a full deletion of this domain. BucLoc $\Delta 64-71$ was expressed at low levels and did not localize to endogenous germ plasm compared to the WT BucLoc (Figure 24 A B, $\underline{\mathrm{C}, \mathrm{F}, \mathrm{G})}$. Therefore, we concluded that both removing the C-terminal PrD completely and introducing the triple D mutation, either alters BucLoc on the structural level or removes an important regulatory sequence resulting in BucLoc degradation.

To further address the role of the C-terminal PrD in germ plasm localization, we did the systematic mapping of BucLoc domain. We found that the $\Delta 62-66$ and $\Delta 67-71$ BucLoc mutants, lacking both parts of the C-terminal PrD, localized similarly to the WT BucLoc (Figure 24 A; Figure 27 E-H). 
In the end, by the systematic mapping of the BucLoc domain, we found that residues 36-51 of Buc were required, whereas residues 52-71 were dispensable for localization to endogenous germ plasm (Figure 25, Figure 26, Figure 27).

Taken together, we concluded that PrDs are not required for germ plasm localization. Additionally, the residues of the C-terminal PrD might be responsible for BucLoc degradation via a posttranslational modification or contain a structural element important for e.g. protein folding. Thus, Buc requires different domains to mediate germ plasm aggregation during oogenesis and localization during embryogenesis. 

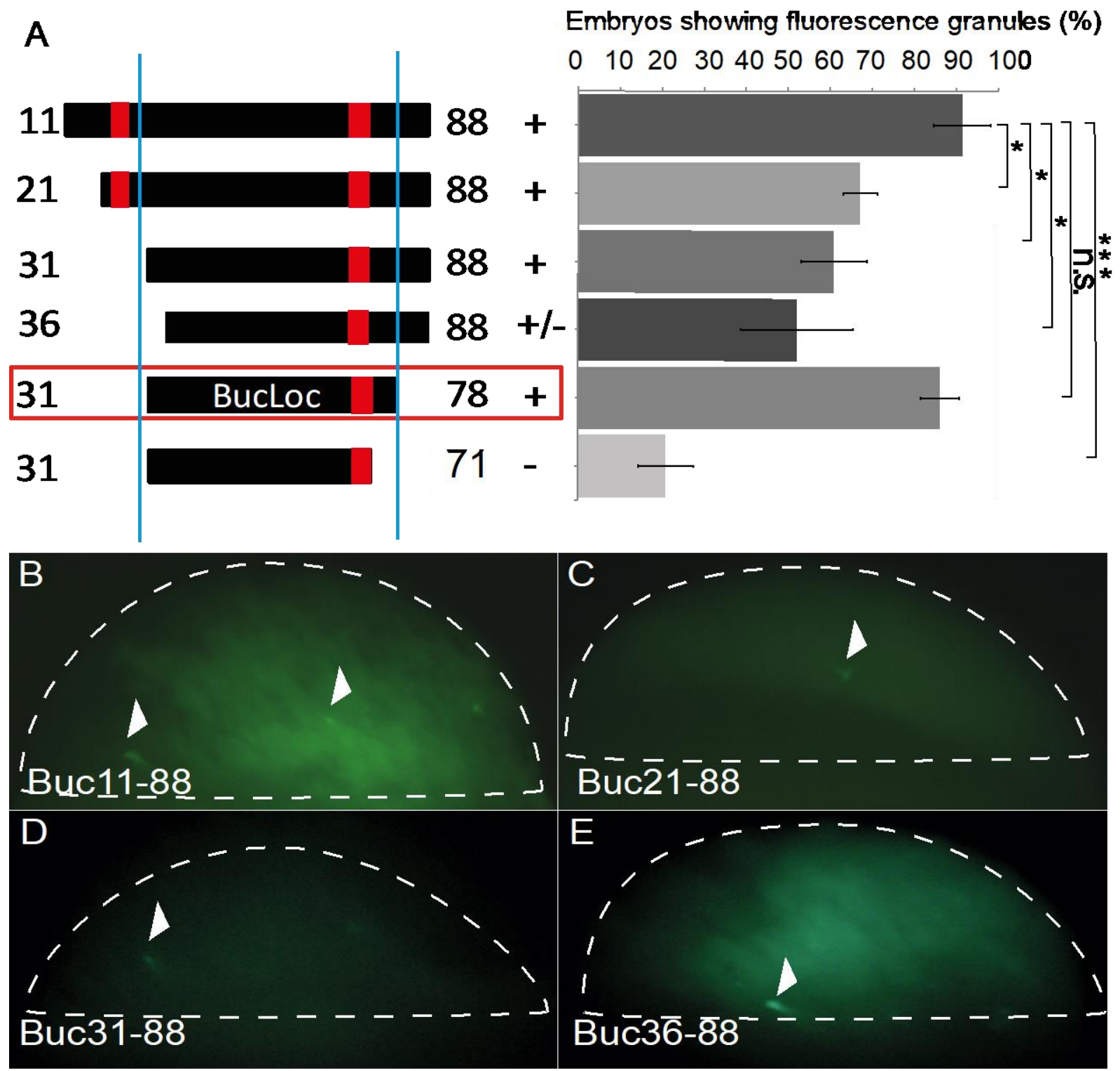

Figure 22: Refined mapping of Buc11-88 localization domain. (A) Summary and quantification of Buc11-88 mapping. Black bar represents size of Buc11-88 deletion constructs in amino acids. "+" stands for localization and "-_-- for no localization. Vertical blue lines highlight $\mathrm{N}$-and C-terminal borders after which further reduction of Buc11-88 results in loss of localization. (+) or (-) stands for localization or no localization. Amyloid domains are shown is red. The minimal

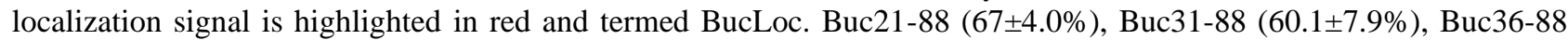
$(52.2 \pm 13.3 \%)$ and Buc31-71 (21.1 $\pm 6.4 \%)$ show significantly less embryos with fluorescent granules than Buc11-88 (91.4 $\pm 6.8 \%)$. Buc31-78 (86.2 $\pm 4.6 \%)$ form fluorescent granules in embryos similarly to Buc11-88. Two tailed student's T-test, Buc11-88 vs the deletion constructs (Buc 21-88 p=0.01; Buc 31-88 p=0.01; Buc36-88 p=0.02; Buc31-78 p=0.41; Buc31-71 $\mathrm{p}=0.0004) ; * P<0.05 ; * * P<0.01 ; * * * P<0.001$ and n.s: not significant. Mean \pm standard deviation is shown. (B-E) Dotted shape outlines embryos at 3-4 hpf in lateral view. Embryos injected with Buc11-88, 21-88, 31-88, 36-88 and 31-78 show localization into a granule (white arrow), whereas Buc31-71 does not localize and show distribution through entire cytoplasm of cells. Scale bar: $50 \mu \mathrm{m}$ 
BuC 11 GVGQPHHPVNHTRPFFYVQPPSQPYFMYQWPMNP-YGHYGFGPALHFGRPYMAPYQFM---QYPGYVIPHAPMQPIDYRR 88

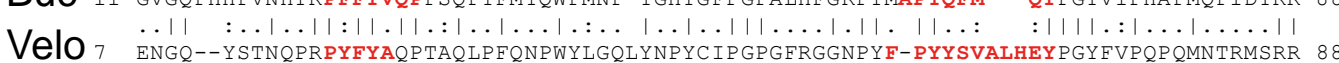

N Q Y G M S P A T F R V I D L K C W E

B Bucky ball WT

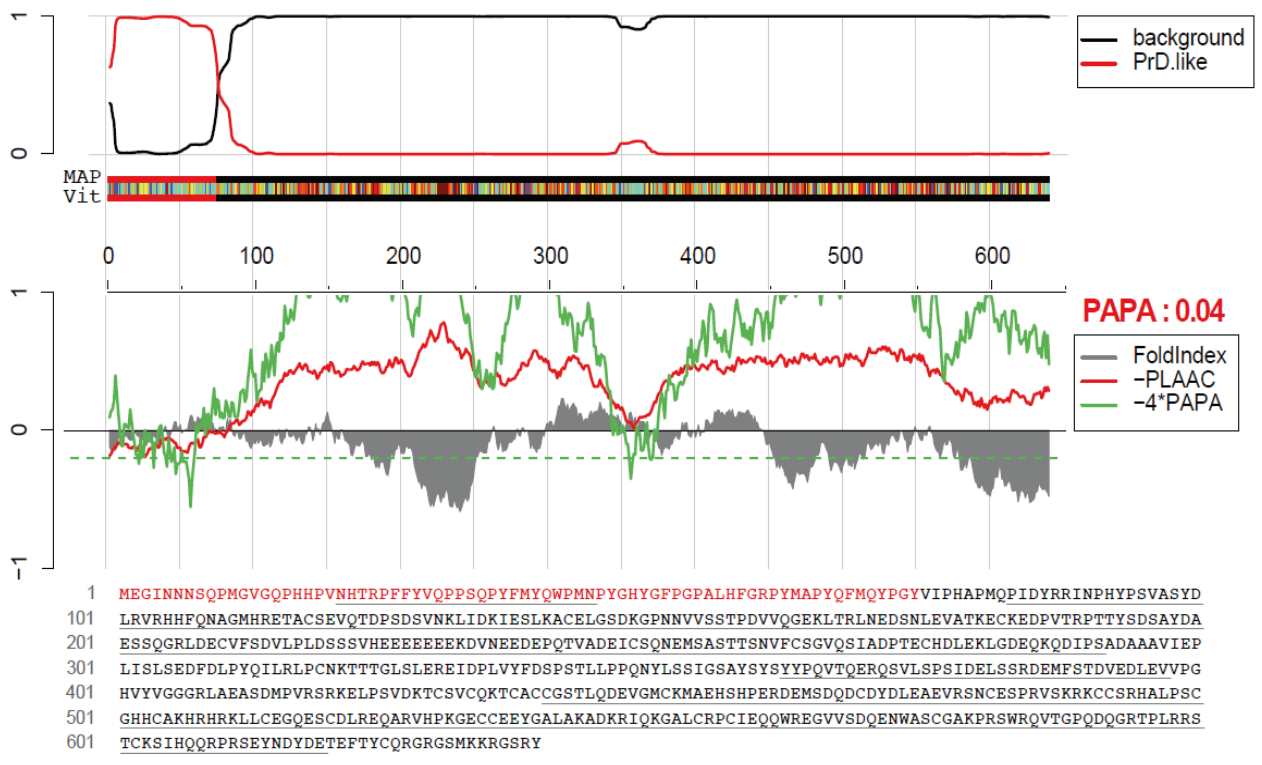

C Bucky ball $-20 \mathrm{~N}+3 \mathrm{D}$

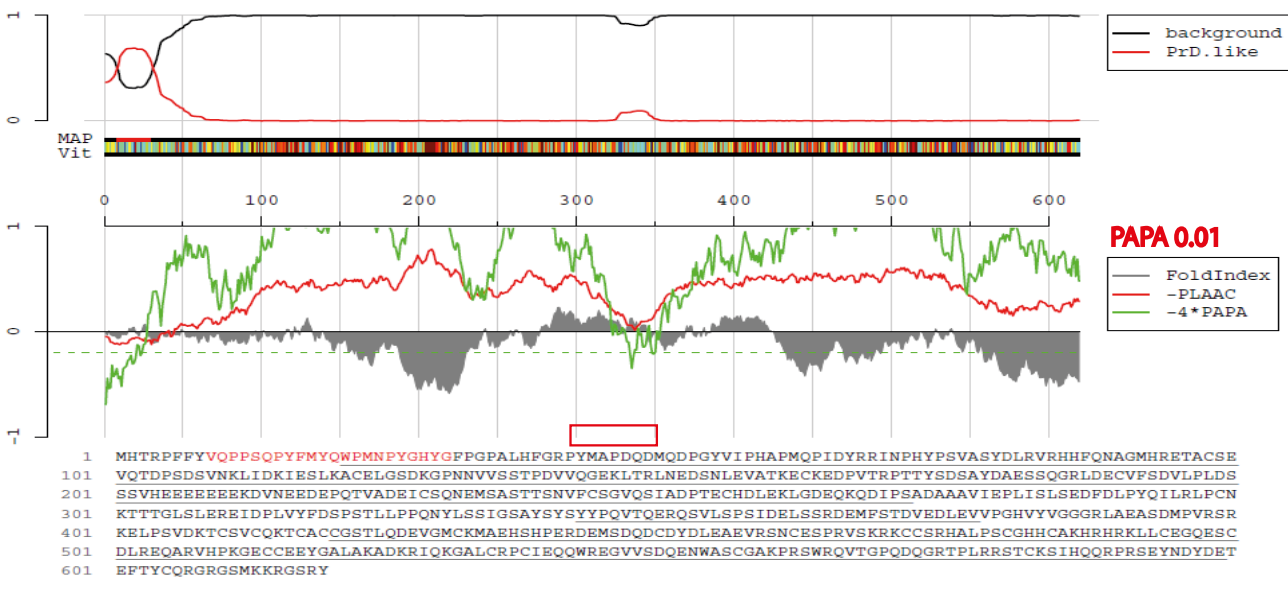

Figure 23: Bucky ball contains predicted prion-like domains. (A) Alignment of Buc11-88 with N-terminus of Velo shows similar predicted aggregation domains (red) (B-C) Visualization PLAAC (Prion-like amino acid composition) outputs: Upper graph in both panels represents a predicted prion like domains (PrDs) (PrD, red line) compared to control (background, black line). Squares with color gradient represent different amino acids in letter code. A second lower graph in both panels represents a propensity of a protein to be prion-like protein vs its disorder. FoldIndex (gray) and -PLAAC (red) represent different ways to visualize regions with prion-like composition predicted as disordered. PAPA is a predicted value for amyloid propensity. PAPA multiplied by -4 ( $-4 *$ PAPA) (green line) is an amyloid prediction value based on a random mutagenesis screen of prion-like proteins. The most negative values of -4 *PAPA predicting best the amyloid propensity (threshold is indicated by the green dotted line). Protein sequence is outlined below, amino acids in red represent the predicted prion-like region. Amino acids in black represent non-prion-like regions. (B) Buc contains $74 \mathrm{~N}$-terminal amino acids which are predicted to form aggregates (PAPA 0.04). In contrast, Buc lacking first N-terminal amino acids (dubbed as Buc $-20 \mathrm{~N}$ ) and containing Y66D, F68D, Y71D mutations (dubbed as 3D), has a shorted predicted prion-like domain of 22 amino acids. Furthermore, Buc $-20 N+3 D$ is predicted to have overall lower PAPA value $(0.01)$ than WT Buc $(0.04)$. 

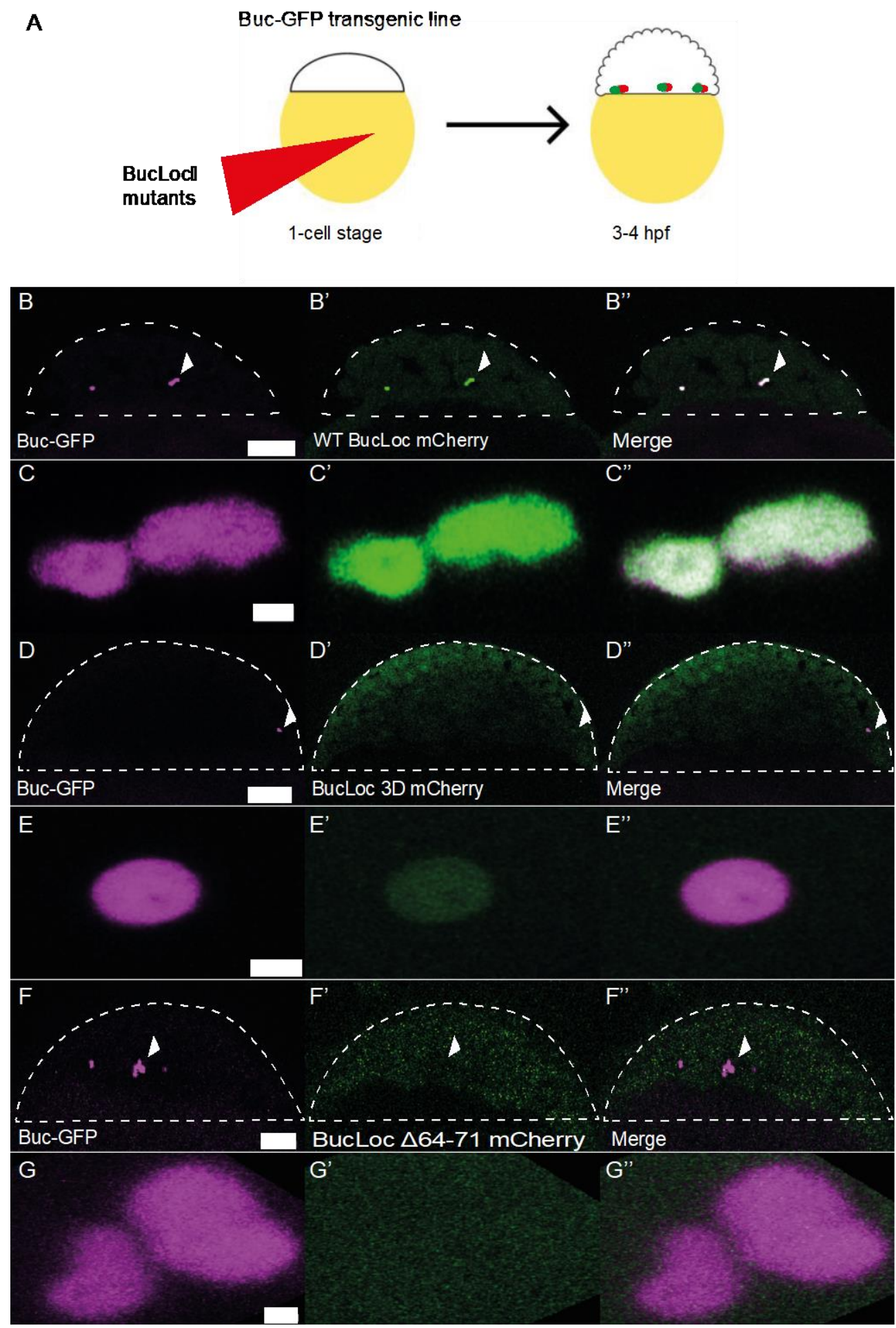

Figure 24: Second prion-like domain in BucLoc is not required for germ plasm localization. (A) A scheme of colocalization assay. BucLoc mCheery fusions were injected into 1-cell stage embryos of the transgenic buc-gfp line. Then at 3-4 hours post fertilization (hpf) embryos were scored for co-localization of injected constructs with Buc-GFP. (B-G) Lateral view of embryos at 3-4 hpf. Dotted half-circle outlines the embryo. White arrowheads show magnified germ plasm in the panel below. Co-localization of BucLoc with transgenic Buc-GFP was determined by live imaging: 1st column - transgenic Buc-GFP (magenta), 2nd column - injected BucLoc mCherry fusion (green) and 3rd column merge. WT BucLoc mCherry fusion co-localizes with transgenic Buc-GFP (B-B', C-C',). Both mCherry fusions of BucLoc 3D and BucLoc $\Delta 64-71$ are expressed at lower levels then WT BucLoc. Only weak co-localization is observed for BucLoc 3D after increasing signal in GFP channel (D-D', E-E'), Co-localization of BucLoc $\Delta 64-71$ with transgenic

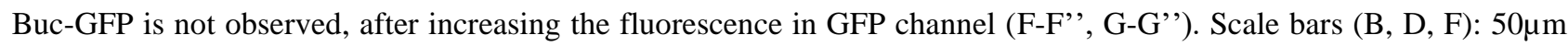
(C, E, G): $2 \mu \mathrm{m}$. 

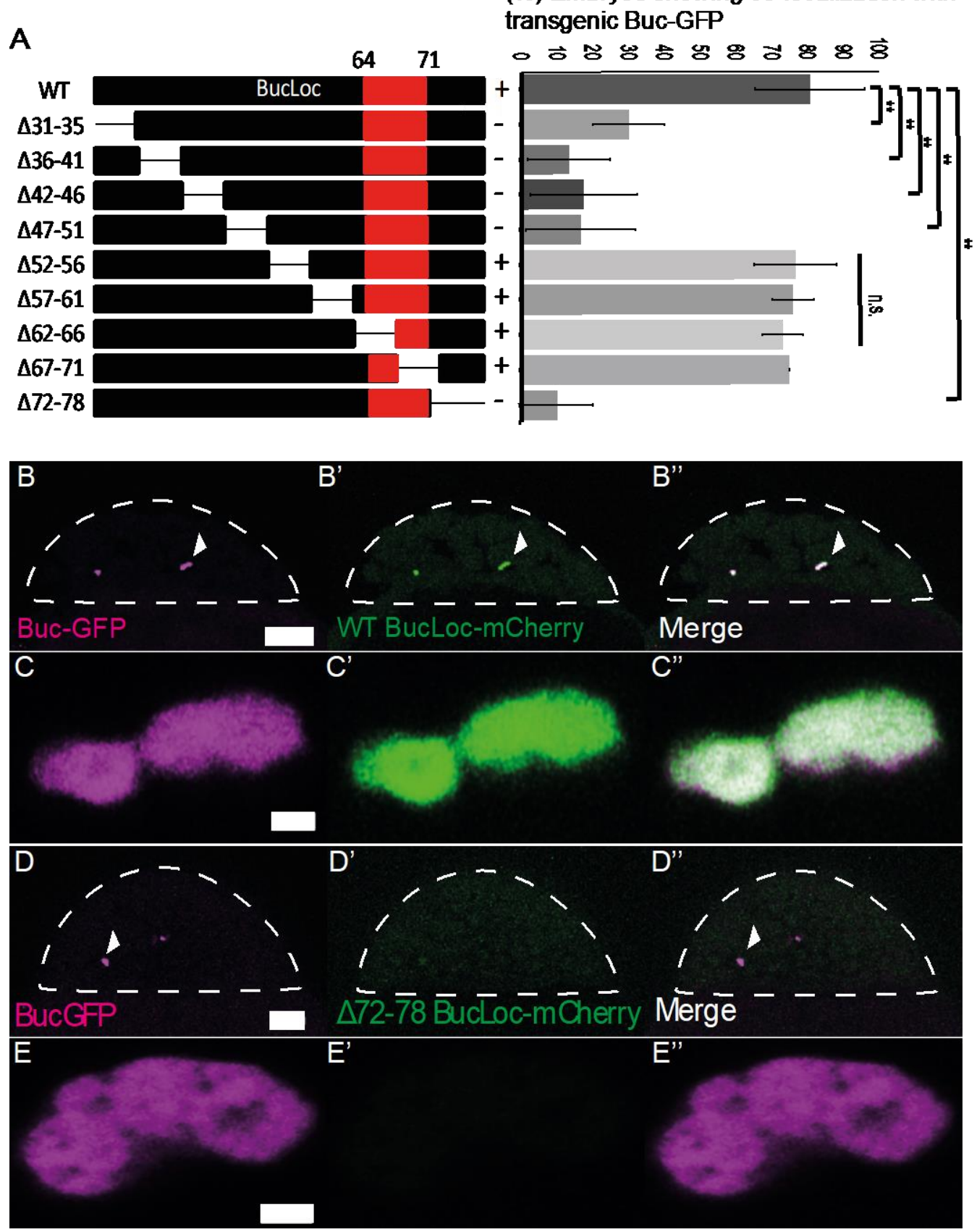

Figure 25: Mapping of BucLoc domain reveals residues important for localization (Part 1). (A) Summary and quantification of BucLoc mapping. Black bar represents size of BucLoc constructs in amino acids. "+" stands for localization and “-“- for no localization. mCherry fusions of BucLoc $\Delta 52-56(76.6 \%, \mathrm{p}=0.4), \Delta 57-61(76.0 \%, \mathrm{p}=0.4)$, $\Delta 62-66(73.0 \%, \mathrm{p}=0.5)$ and $\Delta 67-71(75.0 \%, \mathrm{p}=0.5)$ co-localize with Buc-GFP similarly to WT BucLoc $(80.7 \%)$. In contrast, mCherry fusions of BucLoc $\Delta 31-35$ (30.0\%, $\mathrm{p}=0.009), \Delta 36-41$ (13.3\%, $\mathrm{p}=0.003), \Delta 42-46(17.5 \%, \mathrm{p}=0.005)$, $\Delta 47-51(16.6 \%, \mathrm{p}=0.005)$ and $\Delta 72-78(10.0 \%, \mathrm{p}=0.003)$ co-localize significantly less with Buc-GFP than WT BucLoc. $\mathrm{N}=3, \mathrm{n}=10$. Two tailed student's T-test, BucLoc mutants vs WT BucLoc. Mean \pm standard deviation is shown. $* P<0.05$; $* * P<0.01 ; * * * P<0.001$ and n.s: not significant. (B-E) Lateral view of embryos at 3-4 hpf. Dotted half-circle outlines the embryo. White arrowheads show magnified germ plasm in the panel below. Co-localization of BucLoc with transgenic Buc-GFP was determined by live imaging: $1^{\text {st }}$ column - transgenic Buc-GFP (magenta), $2^{\text {nd }}$ column - injected BucLoc mCherry fusion (green) and $3^{\text {rd }}$ column - merge. WT BucLoc mCherry fusion co-localizes with transgenic Buc-GFP (BB', C-C',) whereas $\Delta 72-78$ BucLoc mCherry fusion does not co-localize (D-D', E-E''). Scale bars (B, D): $50 \mu \mathrm{m}(\mathrm{C}$, $\mathrm{E}): 2 \mu \mathrm{m}$. 


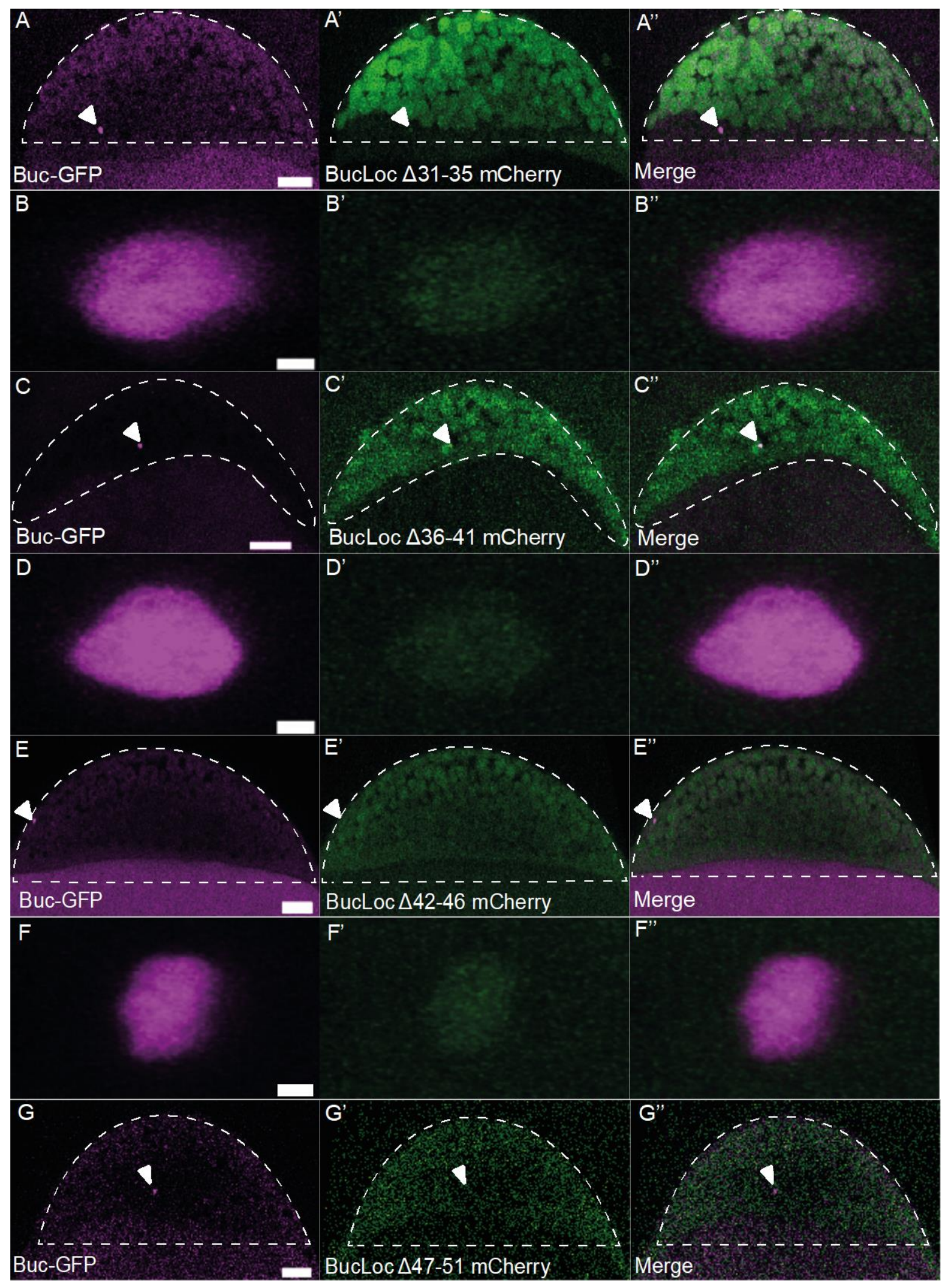

Figure 26: Mapping of BucLoc domain reveals residues important for localization (Part 2). Mapping of BucLoc domain was done by live-imaging of injected mCherry fusions of BucLoc mutants in the Buc-GFP transgenic background. (A, C, E, G) Lateral view of embryos at 3-4 hpf. Dotted half-circle outlines the embryo. Magnified germ plasm indicated by white arrowheads is shown in the panel below (B, D, F, H). Co-localization of BucLoc with transgenic Buc-GFP was determined by live imaging: $1^{\text {st }}$ column - transgenic Buc-GFP (magenta), $2^{\text {nd }}$ column - injected BucLoc mCherry fusion (green) and $3^{\text {rd }}$ column - merge. Injected mCherry fusions of BucLoc $\Delta 31-35, \Delta 36-41, \Delta 42-46$ and $\Delta 47-51$ do not colocalize with transgenic Buc-GFP. Scale bars (A, C, E, G): $50 \mu \mathrm{m}$ (B, D, F, H): $2 \mu \mathrm{m}$. 


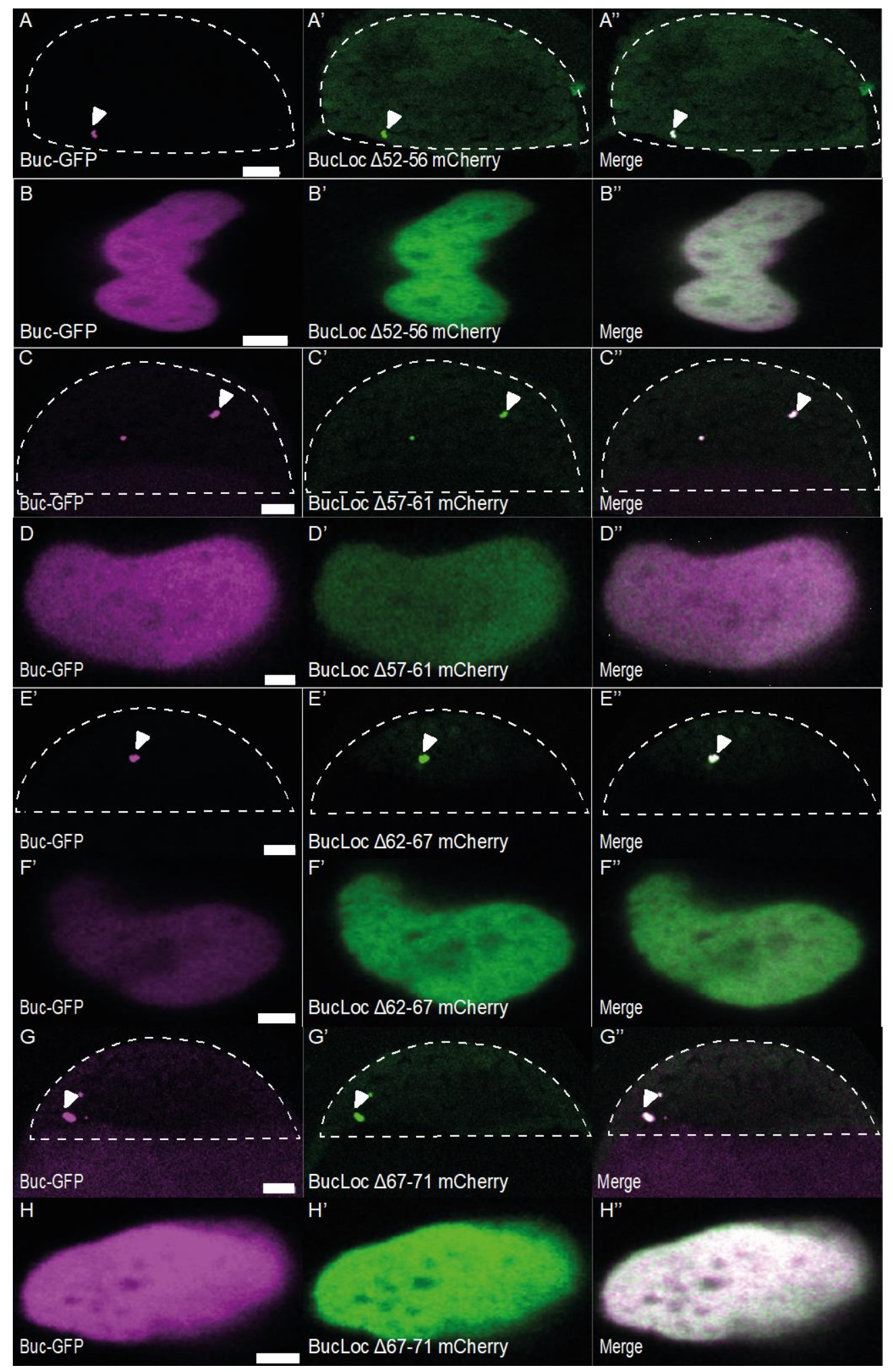

Figure 27: Mapping of BucLoc domain reveals residues important for localization (Part 3). Mapping of BucLoc domain was done by live-imaging of injected mCherry fusions of BucLoc mutants in the Buc-GFP transgenic background. (A, C, E, G) Lateral view of embryos at 3-4 hpf. Dotted half-circle outlines the embryo. Magnified germ plasm indicated by white arrowheads is shown in the panel below (B, D, F, H). Co-localization of BucLoc with transgenic Buc-GFP was determined by live imaging: $1^{\text {st }}$ column - transgenic Buc-GFP (magenta), $2^{\text {nd }}$ column - injected BucLoc mCherry fusion (green) and $3^{\text {rd }}$ column - merge. Injected mCherry fusions of BucLoc $\Delta 52-56, \Delta 57-61, \Delta 62-$ 66 and $\Delta 67-71$ co-localize with transgenic Buc-GFP similarly to WT BucLoc (Figure 20). Scale bars (A, C, E, G): 50 $\mu \mathrm{m}(\mathrm{B}, \mathrm{D}, \mathrm{F}, \mathrm{H}): 2 \mu \mathrm{m}$ 


\subsubsection{BucLoc phosphorylation is not required for germ plasm localization}

We demonstrated that the PrDs are not required for germ plasm localization during zebrafish embryogenesis (Figure 22, Figure $25 \mathrm{~A}$ ). However, we showed that the C-terminal PrD mutant (BucLoc 3D) and the full PrD deletion (BucLoc $464-71$ ) are expressed at low levels (Figure 24 D, E, $\underline{F, G)}$, while they still localized weakly to germ plasm. Therefore, we hypothesized that BucLoc contains elements which regulate Buc localization or degradation. Since BucLoc contains one serine and eight tyrosine residues, we hypothesized that phosphorylation could be one of such regulatory mechanism (Figure $28 \mathrm{~A})$.

We first investigated the role of tyrosine phosphorylation in Buc localization. For this we generated BucLoc phosphomimetic (All Y to E) and non-phosphorylatable (All Y to F) mutants, fused them to mCherry and studied their co-localization with the endogenous germ plasm using the co-localization assay (Figure $24 \mathrm{~A}$ ). BucLoc All Y to E did not co-localize with the endogenous germ plasm (Figure $\underline{28 \mathrm{D})}$. However, BucLoc All Y to E mutant was expressed at low levels and we had to increase signal with Adobe Photoshop (Figure 28 D). Previously, we found that BucLoc 3D mutant was also weakly expressed, therefore we used BucLoc 3D mutant as a negative control in this experiment (Figure 28 $\underline{\mathrm{A}, \mathrm{F}}$. The low expression levels could be the reason why no co-localization with endogenous germ plasm was observed for the BucLoc All Y to E mutant (Figure $28 \mathrm{~A}, \mathrm{D})$. In contrast, BucLoc all Y to F mutant localized to germ plasm similarly to the WT BucLoc (Figure 28 A, B, C). Thus, we assume that tyrosine phosphorylation does not play a role in germ plasm localization, but in BucLoc degradation.

To investigate the role of the single serine in Buc localization, we generated BucLoc phosphomimetic (S33E) and non-phosphorylatable (S33A) mutants. We found that both mutants localized to germ plasm similarly to the WT BucLoc. Taken together, these results indicate that tyrosine or serine phosphorylation of BucLoc domain is not required for germ plasm localization.

To further address the role of phosphorylation in Buc localization, we used $\lambda$ phosphatase to investigate phosphorylation status of BucLoc. $\lambda$ phosphatase is a broadly used enzyme which removes phosphate groups from phosphorylated serine, thereonine and tyrosine (Cohen and Cohen, 1989; Zhuo et al., 1993) To check the status of BucLoc phosphorylation, we injected BucLoc GFP fusion RNA into 1-cell stage embryos. Subsequently we lysed embryos at 3-4 hpf when GFP granules were observed. Then we treated lysates with $\lambda$ phosphatase or only with $\lambda$ phosphatase buffer (control). Subsequently, we performed a western blot to detect BucLoc-GFP. We found that BucLoc-GFP is expressed at similar levels in both the $\lambda$ phosphatase treated and control samples (Figure 30 Lanes 3 and 4). Furthermore, we did not observe any shifts in protein bands upon the $\lambda$ phosphatase treatment. Thus, we confirmed our previous result that BucLoc is not phosphorylated during embryogenesis. Therefore, BucLoc phosphorylation is not required for germ plasm localization.

We further addressed the role of Buc phosphorylation in germ plasm localization by investigating endogenous germ plasm for the presence of phosphotyrosines (Ptyr). For this we performed immunohistochemistry analysis of oocytes and embryos to investigate co-localization of Buc and Ptyr. We found that Buc and Ptyr co-localized during the early and late IB stages of oogenesis in Bb (Figure $31 \mathrm{~A}-\mathrm{C}$ ). In contrast to oogenesis, during embryogenesis we found that the most Ptyr is enriched at cell junctions (Figure $31 \mathrm{D}, \mathrm{E})$.

In summary, we found that BucLoc phosphorylation is not required for localization to germ plasm during embryogenesis. Furthermore, our data suggest the role of tyrosine phosphorylation in Buc 
degradation. This is the first evidence showing the importance of post-translational modifications for the regulation of germ plasm localization in zebrafish.

Emrbyos showing co-localization [\%]

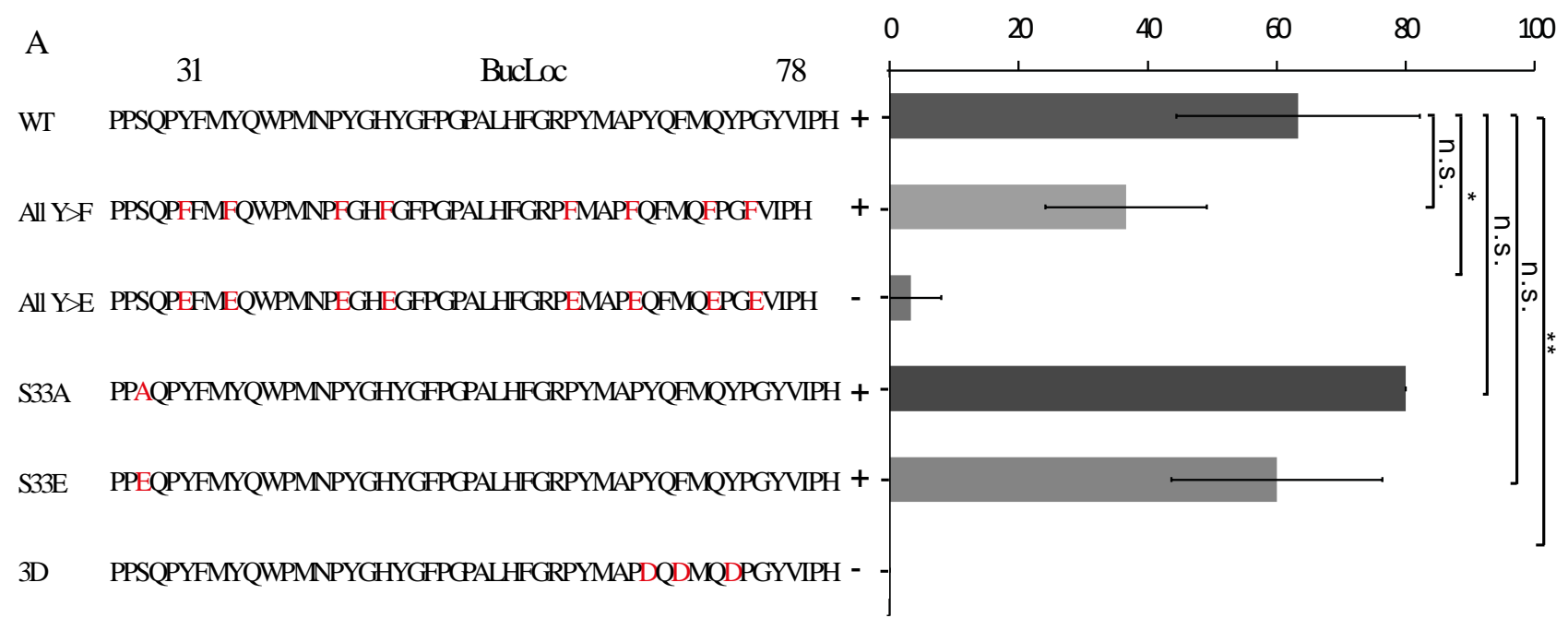

Figure 28: BucLoc phosphorylation is not required for germ plasm localization. (A) Summary and quantification of mapping potential phosphorylation residues in BucLoc. Amino acid sequence of BucLoc is shown in black, mutated residues are shown in red. In order to study BucLoc phosphomutants co-localization with endogenous germ plasm, we injected RNA of BucLoc mCherry fusion into1-cell stage embryos of transgenic Buc-GFP line and scored embryos for co-localization at 3-4 hpf. mCherry fusions of BucLoc all $\mathrm{Y}>\mathrm{F}(36.7 \pm 12.5 \%)$, S33A $(80 \pm 0 \%)$ and S33E $(60 \pm 16.3 \%)$ mutants co-localize with endogenous germ plasm similarly to WT BucLoc $(63.3 \pm 18.9 \%)$. In contrast, mCherry fusions of BucLoc all $\mathrm{Y}>\mathrm{E}(3.3 \pm 4.7 \%, \mathrm{p}=0.01)$ and $3 \mathrm{D}(0 \%, \mathrm{p}=0.009)$ mutants co-localize significantly less frequent with endogenous germ plasm than BucLoc WT. $\mathrm{N}=3, \mathrm{n}=10$. Two tailed student's T-test, BucLoc mutants vs WT BucLoc. Mean \pm standard deviation is shown. ${ }^{*} P<0.05 ; * * P<0.01 ; * * * P<0.001$ and n.s: not significant. 


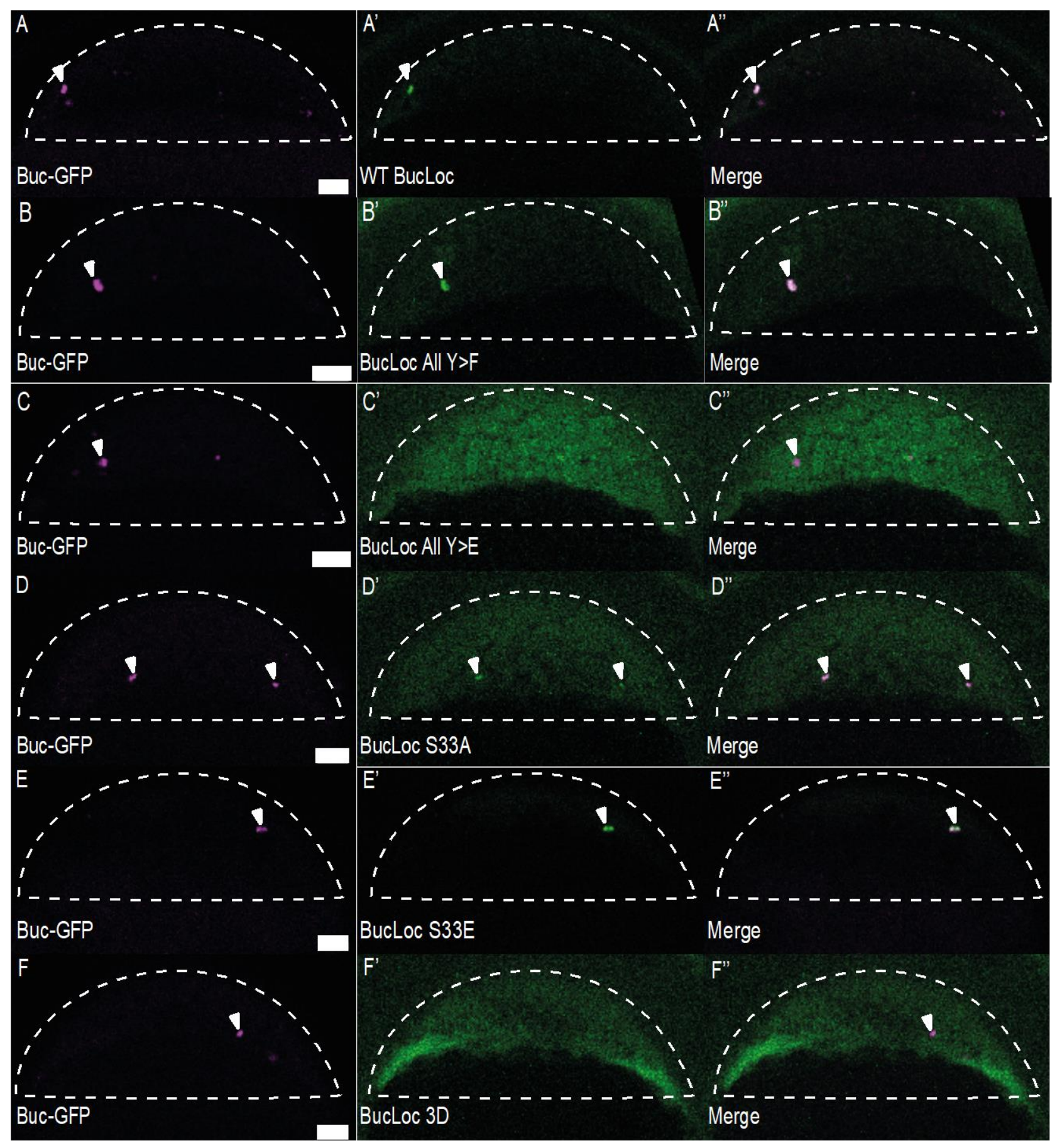

Figure 29: BucLoc phosphorylation is not required for germ plasm localization. In order to study BucLoc phosphomutants co-localization with endogenous germ plasm, we injected RNA of these mutants fused to mCherry into1cell stage embryos of transgenic buc-gfp line. Co-localization of BucLoc WT and mutants with transgenic Buc-GFP was determined by live imaging at 3-4 hpf: 1st column - transgenic Buc-GFP (magenta), 2nd column - injected BucLoc mCherry fusion (green) and 3rd column - merge. Each panel represents an embryo in a lateral view. Dotted half-circle outlines the embryo. White arrowheads point on endogenous germ plasm and BucLoc phosphomutants (when there is a fluorescent granule present). MCherry fusions of BucLoc all Y>F (B-B'), S33A (D-D') and S33E (E-E') mutants colocalize with transgenic Buc-GFP similarly to WT BucLoc (A-A'). In contrast, mCherry fusions of BucLoc all Y>E (CC') and 3D (F-F'") mutants do not co-localize with BucLoc WT. Scale bars: $50 \mu \mathrm{m}$ (F. Mainz, A. Goloborodko) 


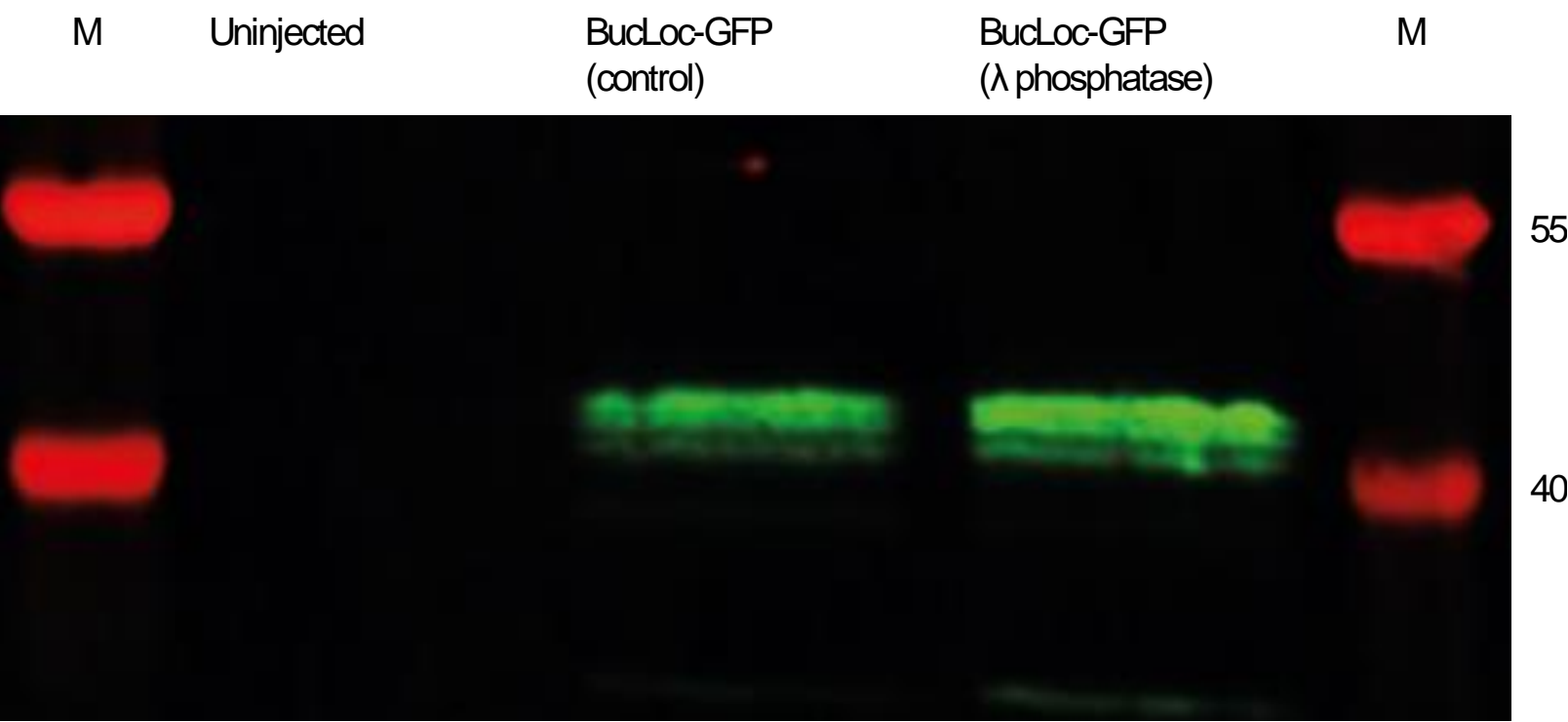

Figure 30: BucLoc phosphorylation is not required for germ plasm localization. To address the role of phosphorylation in germ plasm localization, GFP fusion of BucLoc was injected into 1-cell stage embryos. Then 3-4 hpf embryos were lysed and treated with $\lambda$ phosphatase. Subsequently, a Western blot was performed with the $\lambda$ phosphatase treated embryonic lysates. Untreated BucLoc-GFP lysates were taken as a negative control for the $\lambda$ phosphatase treatment. Uninjected embryos were used as a negative control for the primary anti-GFP antibody specificity. Uninjected sample shows no GFP signal (lane 2), whereas BucLoc-GFP injected samples show multiple bands around $40 \mathrm{kDa}$ (Lane 3 and 4). No difference in protein bands size or number is observed between the $\lambda$ phosphatase treated (lane 4) or the control sample (Lane 3) 


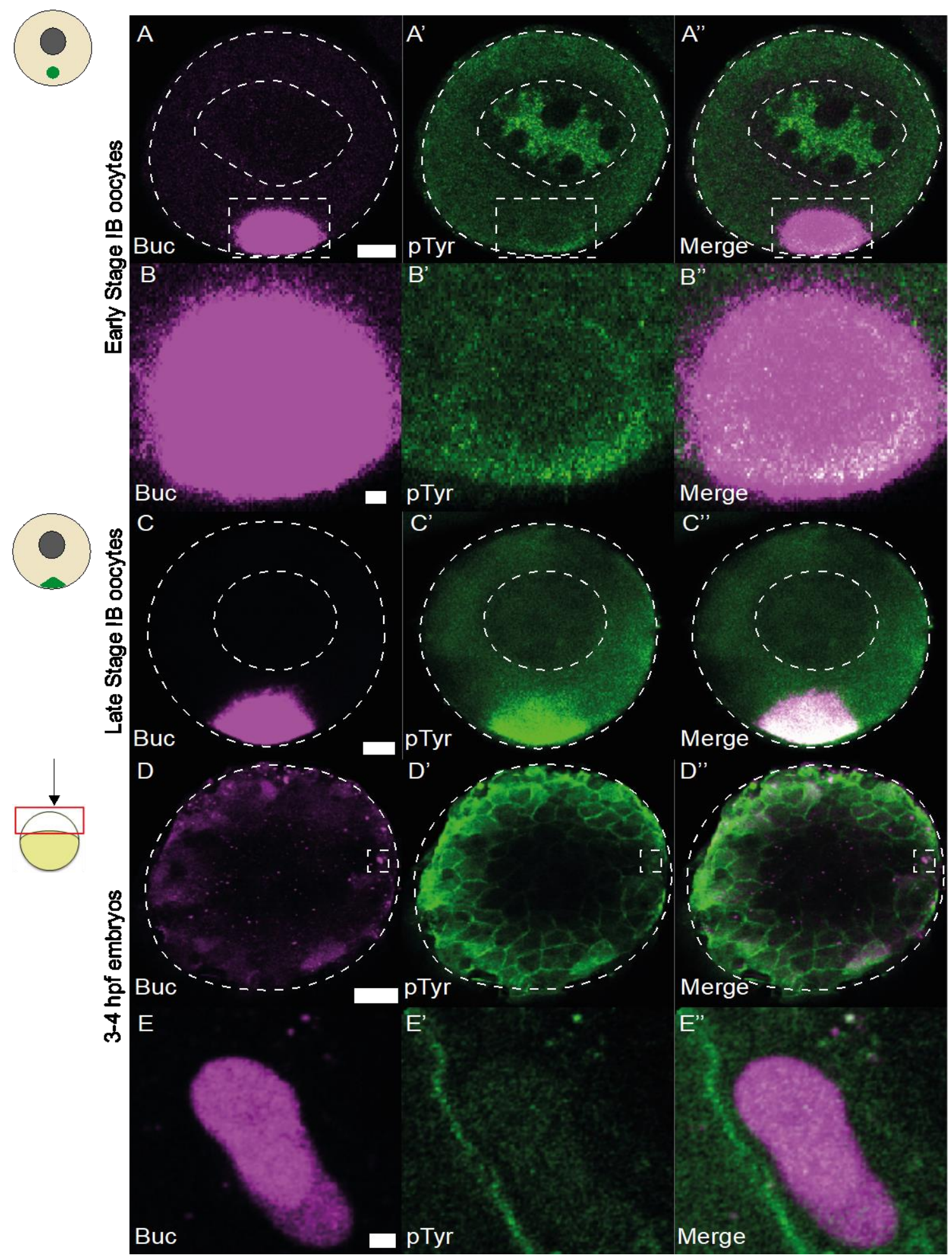

Figure 31: BucLoc phosphorylation is not required for germ plasm localization. Phosphorylation of germ plasm proteins at Tyrosines, was investigated at different stages of Zebrafish development. (A) Early stage IB and (C) late stage IB oocytes. Dotted rectangle in (A) represents magnified Bb in (B). (D) Animal view of embryos at 3-4 hpf. Dotted circle outlines the embryo. Dotted rectangle outlines magnified germ plasm in (E). Presence of proteins containing pTyr in germ plasm was determined by immunohistochemistry: $1^{\text {st }}$ column - endogenous Bucky ball (Buc) (magenta), $2^{\text {nd }}$ column phosphotyrosine (pTyr) (green) and $3^{\text {rd }}$ column (merge). Ptyr is enriched in the nucleus and in the outer layer of $\mathrm{Bb}$ in the early sage IB oocytes (A-A', B-B' '). Furthermore, Ptyr enriched in Bb co-localizes with Buc (B-B' '). (C-C') In the late stage IB oocytes, there is more Ptyr signal present in Bb than during the early IB stage oogenesis (A-A'). Furthermore, Buc fully co-localizes with Ptyr during the late stage IB oogenesis (C-C' '). In the 3-4 hpf embryos, Ptyr is enriched at the cell junctions, but not in germ plasm (D-D', E-E' ). Scalebars: (A, C) $5 \mu \mathrm{m}$; (B, E) $2 \mu \mathrm{m}$; (D) $50 \mu \mathrm{m}$. 


\subsection{Identification of the core BucLoc interactome}

Buc protein provides us a molecular proxy which can be used to study the molecular mechanisms of germ plasm localization. Recently, isolation of the Buc localization signal allowed a biochemical screen which identified proteins directly involved in germ plasm localization (Riemer 2014, Doctoral dissertation). The biochemical screen revealed 213 potential interactors of the Buc localization domain. Based on such long candidate list, the selection and investigation of novel proteins involved in Buc localization is time laborious and inefficient. Therefore, it is important to repeat the biochemical screen and to find the core interactors of Buc which play a role in germ plasm localization.

\subsubsection{Identification of the core BucLoc interactome}

Previously we mapped the localization signal of Buc (aa 11-88) to identify the smallest localization motif possible (Figure 22). The mapping resulted in identification of the $47 \mathrm{~N}$-terminal Buc residues which are sufficient for germ plasm localization. We dubbed this N-terminal motif as BucLoc. We used BucLoc as a bait for a biochemical screen to identify interactors involved in germ plasm localization.

In order to perform the biochemical screen, we injected bucloc-fgp RNA into 1-cell stage embryos and investigated embryos for presence of fluorescent granules at $3 \mathrm{hpf}$ (Figure $32 \mathrm{~A}$ ). Then, embryos were lysed and BucLoc-GFP co-immunoprecipitation (co-IP) was accomplished with GFP-binding nanobodies. Subsequently, mass spectrometry (MS) analysis was performed. Two negative controls were used in this analysis to exclude irrelevant BucLoc interactors. Firstly, co-IP was performed with transgenic $g f p$ embryos. Secondly, co-IP was performed with injected bucloc $472-78 f g p$, which localizes less efficiently to germ plasm than the WT BucLoc-GFP (Figure 25). As a positive control, co-IP was done with transgenic buc-gfp embryos. Transgenic Buc-GFP is expressed at endogenous levels and therefore contains all endogenous Buc interactors (Riemer et al., 2015). Prior to the MS analysis, western blot was performed to check Buc co-IP samples for presence of Vasa. Previously, we demonstrated that Vasa protein interacts with Buc (Krishnakumar et al., 2018).

The western blot with input and co-IP samples showed that all samples contained intact proteins prior to the co-IP (Figure 32 B). After the co-IP we also detected all GFP (fusion) proteins (Figure $32 \mathrm{~B}$ ). Hence, the pull-down with the GFP-binding nanobodies worked. Furthermore, we detected Vasa protein only in the Buc-GFP sample, but not in the other samples, as expected. This result is in line with Vasa interaction exclusively with the C-terminal part of Buc (Perera, unpublished). We also attempted to validate BucLoc-GFP interaction with ZO1, ZO2 and p-NMII proteins, which were enriched in the previous MS analysis (Riemer 2014, Doctoral dissertation). However, we were unable to detect these proteins by a western blot in the input or co-IP samples (data not shown). After performing the control experiments, we proceeded with the MS analysis of the co-IP samples.

The MS analysis identified 291 proteins and after the bioinformatics we obtained 23 proteins (Figure $32 \mathrm{C}$; Table 1). These candidate proteins were assigned to their most relevant function and cellular compartment based on the data available at the UniProt database (Figure 33) (Bateman, 2019). Among all candidates we found two well studied germ plasm specific proteins: Piwi-like protein 1 (Piwil1) and tudor domain-containing protein 6 (Tdrd6). Piwil1 maintains germline integrity during gametogenesis by repressing transposable elements (Houwing et al., 2007), whereas tudor domaincontaining proteins are involved in regulation of Buc aggregation (Roovers et al., 2018). Both Tdrd6 
and Piwill homologs in zebrafish: Ziwi and Zili localize to germ granules (Roovers et al., 2018; Tan et al., 2002). Hence, this result shows the specificity of the biochemical screen to isolate germ plasm proteins. Furthermore, we found DEAD (Asp-Glu-Ala-Asp) box helicase (Ddx5), which is different from Vasa protein (Ddx4) used as our positive control for the Buc-GFP co-IP. Since Piwill and Ddx5 are the most enriched proteins compared to GFP (Figure 33, Table 1), we think that Ddx5 is also part of zebrafish germ plasm. Among the most enriched proteins compared to GFP control we also found insulin-like growth factor 2 mRNA-binding protein 3 (Igf2bp3) (Figure 33, Table 1). However, it is unlikely that the most enriched proteins: Ddx5, Piwill or Igf2bp3 play a role in germ plasm localization, because these proteins are not known to interact with cytoskeleton.

In the next step, we focused on the candidate proteins enriched most in BucLoc-GFP compared to the BucLoc $472-78$ GFP control. We found nuclease-sensitive element-binding protein 1 isoform 2 (Nsebp1), Bucky ball 2-like (Buc2l) and si:rp71-45k5.4. Nsebp1 and si:rp71-45k5.4 are proteasome components and are unlikely to play a role in germ plasm localization (Bateman, 2019). Interestingly, Buc and Buc2l have only 28\% similarity. However, the function of Buc2l in germ plasm is unknown and we cannot exclude a role of Buc2l in germ plasm localization.

The largest group (Figure 33, $\mathrm{n}=6$, green) of the identified proteins are mainly involved in RNA binding, transport and storage. Interestingly, from these candidate proteins, Intracellular hyaluronanbinding protein 4 (Ihabp4) is able to both bind RNA and intracellular receptor hyaluronan in chick and mouse (Huang et al., 1999). Hyaluronan is a unique glycosaminoglycan, which links hyaluronanbinding proteins to plasma membrane. However, the function of Ihabp4 remains unexplored. Therefore, Ihabp4 is a promising candidate for anchoring germ plasm to cytoskeleton.

We also identified a group of uncharacterized proteins (Figure 33, n=3, blue) including a possible component of germplasm - Buc2l protein and two other proteins: Ras-GTPase activating protein SH3 domain-binding protein 2-like (a homolog of human G3bp2) and RNA binding motif protein 4.3 (Rbm4.3.). G3bp2 is a conserved component of stress granules and is important for post-translation modifications of subset of RNAs (Irvine et al., 2004). However, the function of G3bp2 is not yet entirely clear, especially in zebrafish. Rbm4.3. is predicted to be an RNA binding protein. Furthermore, we found two subsets of proteins which play a role in mRNA translation (Figure 33, $\underline{\mathrm{n}=4 \text {, orange) }}$ and in proteasomal degradation (Figure 33, $\mathrm{n}=4$, brown). Finally, we also identified one protein, responsible for protein transport in endoplasmic reticulum (Figure 33, gray) and one chaperon protein, responsible for protein folding in mitochondria (Figure 33, yellow).

In conclusion, from the identified BucLoc core interactors, Ihabp4 is the most promising candidate for anchoring germ plasm to cytoskeleton. Furthermore, G3bp2, Rbm4.3. and Buc2l are less likely to play a role in germ plasm localization, because they have no known domains or data on interaction with cytoskeleton. 
A

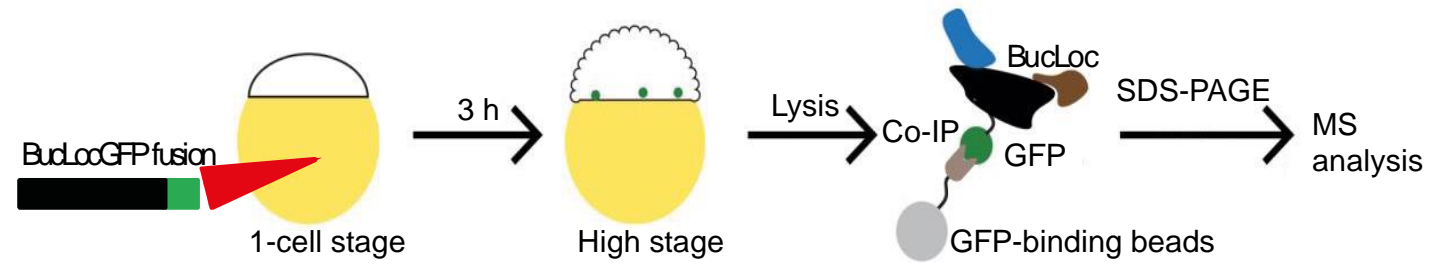

B

Input

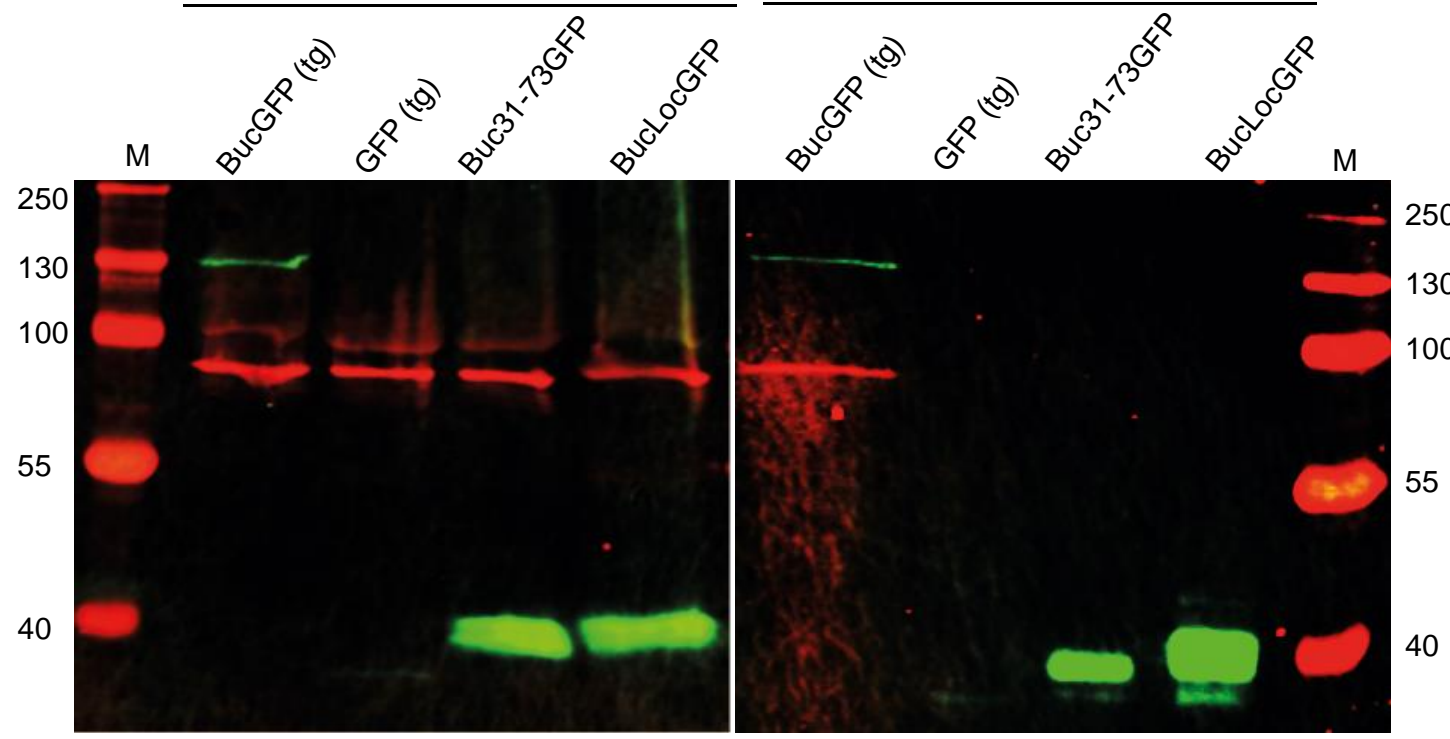

C Number of interaction candidates

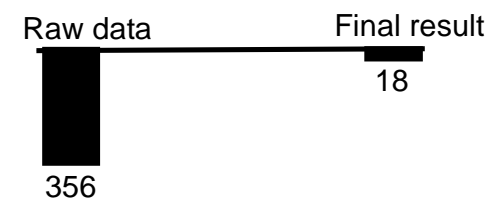

BucLocGFP > 2x GFP

BucLccGFP > 2x Buc31-73GFP

BucLocGFP $\approx$ BucGFP

Figure 32: BucLoc interactome analysis. (A) A scheme of a method to screen for BucLoc interactors. GFP fusion of BucLoc was injected into 1-cell stage embryos. Then, embryos were lysed and co-immunoprecipitation of injected protein was performed. Subsequently, mass spectrometry (MS) analysis was done. Two negative controls were used in this analysis: GFP from transgenic embryos and injected Buc31-73-GFP, which localizes less efficiently to germ plasm then BucLoc-GFP. As a positive control, transgenic Buc-GFP was coimmunoprecipitated and included in the MS analysis. (A-B) Prior to the MS analysis, Western blot (WB) was performed to validate the samples for presence of the overexpressed or transgenic proteins and to check if the coimmunoprecipitation was successful. (B) GFP fusions were visualized by anti-GFP antibody (green) and endogenous Vasa was visualized by anti-Vasa antibody (red). M stands for size marker, IP - for coimmunoprecipitation. WB with input samples show presence of Buc-GFP (lane 2, 130 kDa), GFP (lane 3, 27 $\mathrm{kDa}$ ), Buc31-73GFP (lane 4, 40kDa) and BucLoc-GFP (lane 5, 40kDa). Furthermore, input samples show presence of Vasa (83 kDa, lanes 2-5). Vasa interacts with C-terminus of Buc and therefore is used as a positive control for IP in this analysis. After IP, Vasa is found in BucGFP sample IP (Lane 6), but not in GFP, Buc3173GFP or BucLoc-GFP IPs (Lanes 7-9). (C) A scheme showing pipeline of raw MS data analysis to select the most potential BucLoc interactors. The MS candidates were selected which were at least two-fold more enriched in BucLoc-GFP compared to GFP or Buc31-73GFP. Furthermore, the proteins were selected which were enriched within \pm 4 fold in BucLoc compared to Buc-GFP. 


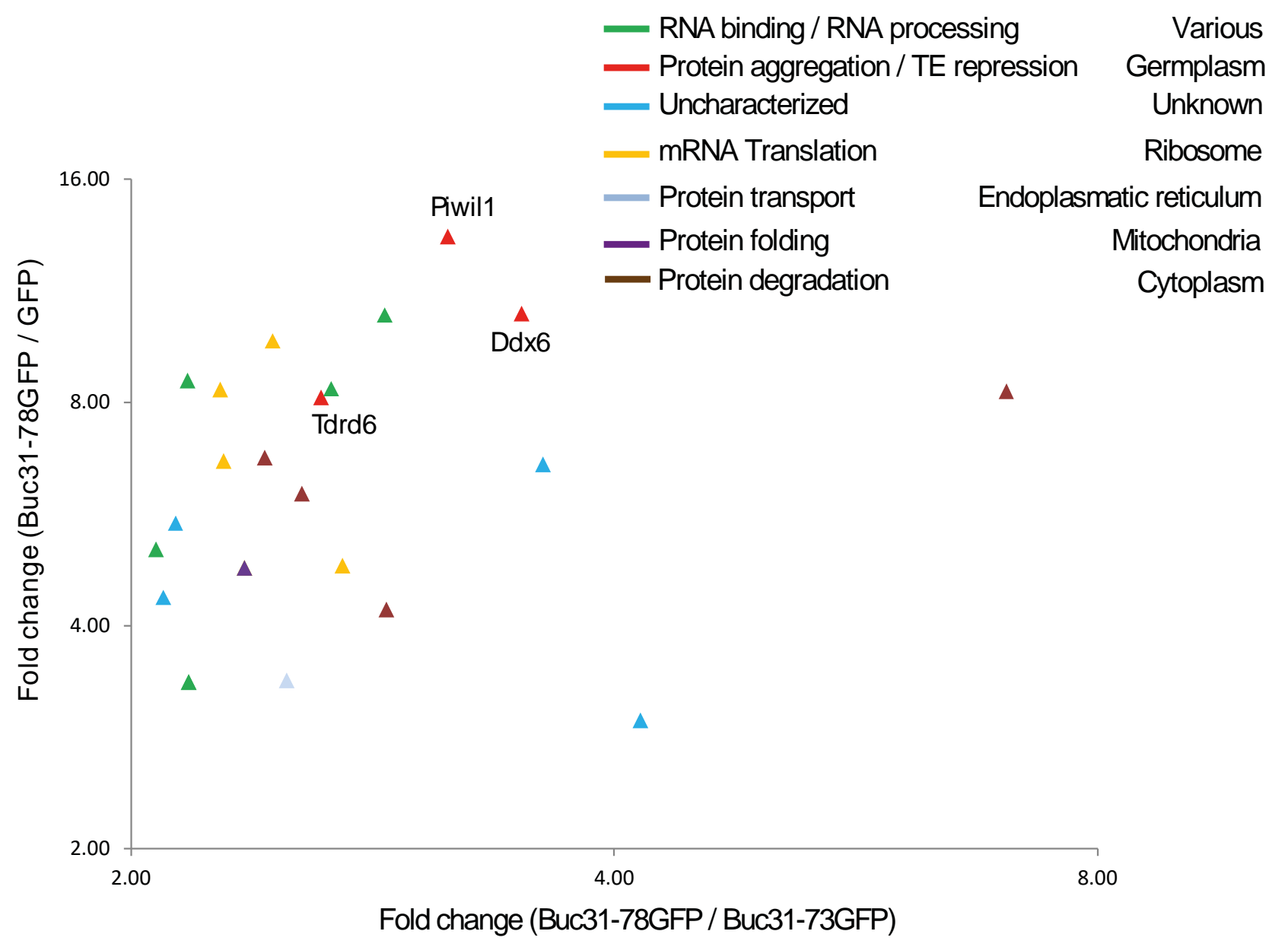

Figure 33: Results of BucLoc interactome analysis. After applying bioinformatics, the most promising 23 candidates are shown. Y-axis represent $\log 2$ of BucLocGFP/GFP fold change, whereas X-axis represents Log2 of BucLoc/Buc3173GFP fold change. Candidates are assigned to different function or compartment based on the data available at the UniProt database (figure legend). HABP2, G3BP ortholog and Buc 2-like were selected for further analysis (encircled in blue). 
Table 1: The core BucLoc interactome. Fold enrichment to GFP, Buc-GFP and Buc31-73GFP in comparison to BucLoc-GFP is given for each candidate. Different colors correspond to protein function and compartment illustrated in Figure 26.

\begin{tabular}{|c|c|c|c|c|c|}
\hline & & & Fold e & richme & \\
\hline Name & Gene & $\begin{array}{l}\text { Human } \\
\text { homolog }\end{array}$ & GFP & $\begin{array}{l}\text { Buc- } \\
\text { GFP }\end{array}$ & $\begin{array}{l}\text { Buc } \\
31- \\
73- \\
\text { GFP }\end{array}$ \\
\hline piwi-like protein 1 & piwill & PIWIL1 & 13.37 & 2.3 & 3.2 \\
\hline $\begin{array}{l}\text { DEAD (Asp-Glu-Ala-Asp) box } \\
\text { helicase } 3 \text { b isoform X5 }\end{array}$ & $d d x 6$ & DDX6 & 10.53 & 3.0 & 3.5 \\
\hline $\begin{array}{l}\text { insulin-like growth factor } 2 \\
\text { mRNA-binding protein } \\
\text { isoform X1 }\end{array}$ & $i g f 2 b p 3$ & $I G F 2 B P 3$ & 10.48 & 3.3 & 2.9 \\
\hline 60S ribosomal protein L12 & rpl12 & RPL12 & 9.68 & 3.2 & 2.5 \\
\hline $\begin{array}{l}\text { polyadenylate-binding protein } \\
\text { 1-like }\end{array}$ & pabpcll & $P A B P C 1$ & 8.56 & 2.6 & 2.2 \\
\hline $\begin{array}{l}\text { poly }(\mathrm{A}) \text { binding protein, } \\
\text { cytoplasmic 1a isoform X1 }\end{array}$ & pabpn1l & $P A B P N$ & 8.34 & 2.6 & 2.7 \\
\hline $\begin{array}{l}\text { 60S acidic ribosomal protein } \\
\text { P0 }\end{array}$ & rplpO & $R P L P O$ & 8.31 & 2.9 & 2.3 \\
\hline $\begin{array}{l}\text { nuclease-sensitive element- } \\
\text { binding protein } 1 \text { isoform } 2\end{array}$ & Ybxl & $Y B X 1$ & 8.28 & 2.6 & 7.0 \\
\hline $\begin{array}{l}\text { tudor } \quad \text { domain-containing } \\
\text { protein } 6 \text { isoform X3 }\end{array}$ & tdrd6 & TDRD6 & 8.11 & 2.3 & 2.6 \\
\hline $\begin{array}{l}\text { 26S protease regulatory subunit } \\
7\end{array}$ & psmd9 & PSMD9 & 6.73 & 3.1 & 2.4 \\
\hline $\begin{array}{l}\text { eukaryotic translation initiation } \\
\text { factor } 3 \text { subunit L }\end{array}$ & eif $3 l$ & EIF3L & 6.67 & 2.8 & 2.3 \\
\hline si:rp71-45k5.4 & $\begin{array}{l}\text { si:rp71- } \\
45 \mathrm{k} 5.4\end{array}$ & PSMA2 & 6.59 & 3.0 & 3.6 \\
\hline $\begin{array}{l}26 \mathrm{~S} \text { proteasome non-ATPase } \\
\text { regulatory subunit } 2\end{array}$ & Psmd2 & PSMD2 & 6.02 & 3.9 & 2.6 \\
\hline
\end{tabular}




\begin{tabular}{|c|c|c|c|c|c|}
\hline $\begin{array}{l}\text { Ras-GTPase activating protein } \\
\text { SH3 domain-binding protein } 2- \\
\text { like }\end{array}$ & Zgc:56304 & $G 3 B P 2$ & 5.49 & 3.1 & 2.1 \\
\hline 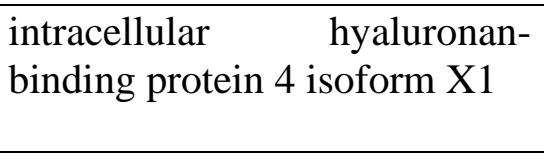 & Zgc: 103482 & HABP4 & 5.07 & 1.8 & 2.1 \\
\hline 60 S ribosomal protein L38 & $R p l 38$ & RPL38 & 4.81 & 2.8 & 2.7 \\
\hline stress -70 protein & Hspa9 & HSPA9 & 4.79 & 2.6 & 2.4 \\
\hline RNA binding motif protein 4.3 & $\operatorname{rbm} 4.3$ & RBM4 & 4.36 & 3.6 & 2.1 \\
\hline $\begin{array}{l}\text { 26S protease regulatory subunit } \\
10 \mathrm{~B}\end{array}$ & Psmc6 & PSMC6 & 4.20 & 2.5 & 2.9 \\
\hline $\begin{array}{l}\text { protein transport } \text { protein } \\
\text { Sec16A isoform X3 }\end{array}$ & Secl6a & SEC16A & 3.37 & 1.8 & 2.5 \\
\hline $\begin{array}{l}\text { KH domain-containing, RNA- } \\
\text { binding, signal transduction- } \\
\text { associated protein } 1\end{array}$ & khdrbsla & KHDRBS1 & 3.35 & 2.0 & 2.2 \\
\hline $\begin{array}{l}\text { uncharacterized protein buc2l } \\
\text { [Danio rerio] }\end{array}$ & Buc2l & $K D M 3 A$ & 2.98 & 2.4 & 4.2 \\
\hline
\end{tabular}




\subsection{Conserved functions of Bucky ball}

We showed that molecular mechanism of germ plasm localization involves tight junction proteins like ZO1 (Chapter 2.1). However, it is unknown whether the mechanisms of PGC induction and germ plasm localization are conserved between vertebrates and invertebrates.

\subsubsection{Mechanism of primordial germ cells induction is conserved in vertebrates and invertebrates}

Previously, we demonstrated that Buc is required for germ plasm assembly and formation of ectopic PGCs (Bontems et al., 2009). Likewise, it was shown that sOsk is required for germ plasm assembly and its mislocalization induces ectopic PGCs (Ephrussi \& Lehmann, 1992; Smith et al., 1992). To understand if the mechanism of PGCs induction of Buc and sOsk is conserved between vertebrates and invertebrates, we overexpressed both germ plasm organizers in the 16-cell assay. In this assay, buc or sOsk RNA together with germ line reporter gfp-nos3'UTR were injected into a corner blastomere of the 16-cell stage embryo (Figure $34 \mathrm{~A}$ ). The corner cell normally does not contain germ plasm and does not acquire PGC fate. However, surprisingly buc RNA injection in the corner blastomere results in formation of ectopic germ cells (Bontems et al., 2009). As a negative control for germ cell induction, a buc mutant (Buc 1-361) was injected, which is unable to assemble germ plasm (Bontems et al., 2009). To confirm the germ cell identity of induced PGCs, injected embryos were checked for presence of Vasa and GFP positive cells at the 15-somite stage. In the 16-cell assay, Vasa highlights the endogenous PGCs, while GFP shows the induced PGCs. Surprisingly we found that sOsk induces formation of ectopic PGCs in zebrafish, similarly to Buc (Figure 34 B-B ', D-D' '), whereas Buc mutant lacked its PGC inducing ability and only showed weak GFP signal in Vasa positive cells (Figure $34 \mathrm{C}$ ). Furthermore, some PGCs showed presence of Vasa, but absence of GFP signal (Figure 34 B-B', D-D', white arrowheads). Therefore next to the induced PGCs, we could also detect endogenous PGCs in the 16-cell assay. Thus, the cells specified by Buc and sOsk differentiate into PGCs. 
A
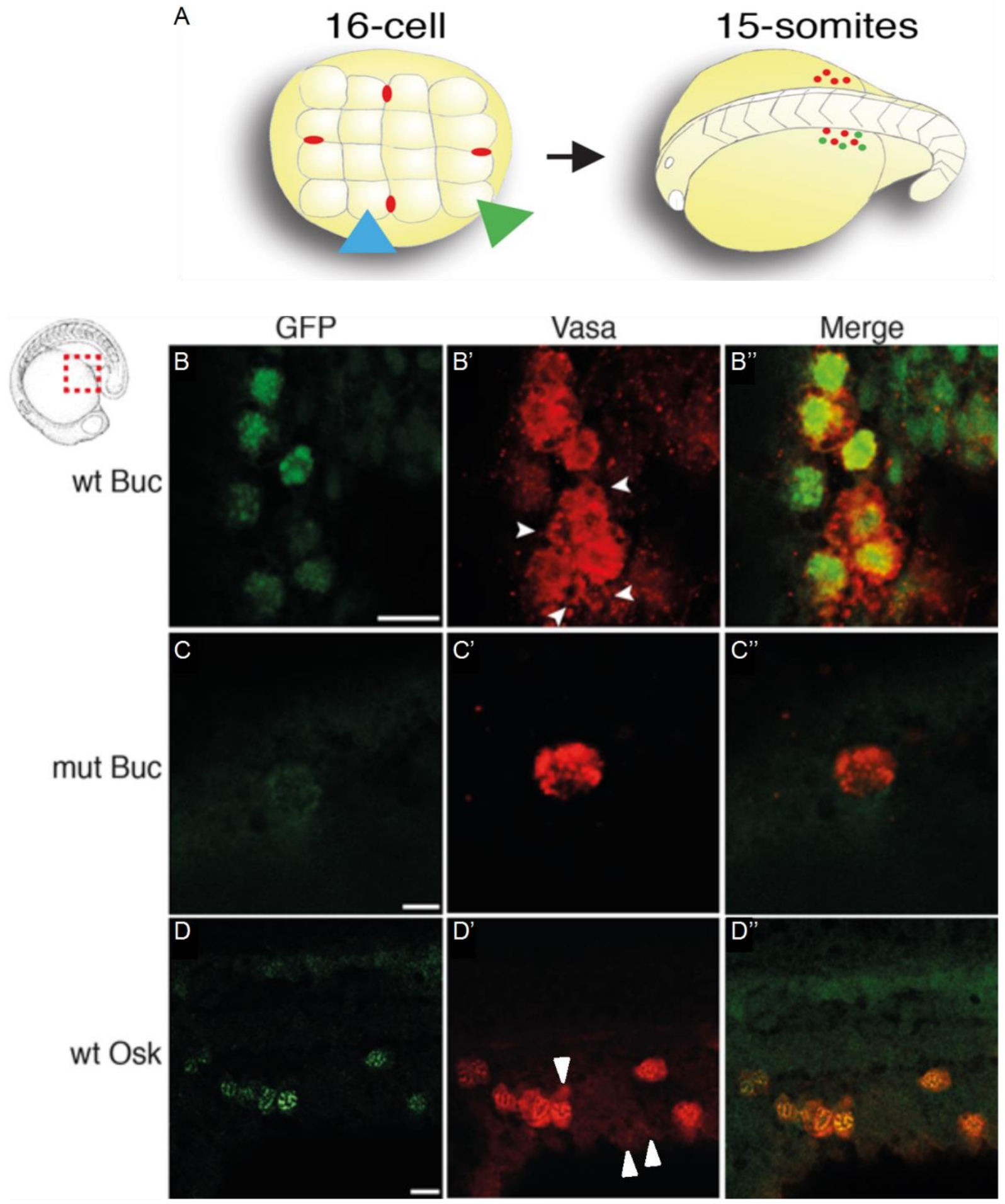

Figure 34: Mechanism of primordial germ cells induction is conserved in vertebrates and invertebrates. (A) Scheme of germ cell induction assay. Left panel: Animal view of a 16-cell embryo injected with PGC-reporter into a middle blastomere (blue arrowhead) containing endogenous germ plasm (red dots) or into a somatic cell (corner blastomere; green arrowhead). Right panel: Oblique, dorsal view of a 15-somite stage embryo (18 hours post fertilization, hpf), anterior to the left. Fluorescent germ cells (white arrowhead) emerge by targeting the reporter to a PGC or transforming a somatic cell into a PGC. Lateral view, anterior to the left of area indicated in icon of 18-somite stage embryo after 16-cell assay with WT Buc(1-639) (B-B"), mutant Buc(1-361) (C-C") or sOsk (D-D'). Embryos were analyzed for GFP (green) and Vasa (red) protein expression. Arrowheads indicate endogenous PGCs (Vasa positive and GFP negative). Scale bar: $20 \mu \mathrm{m}$. (Perera (16-cell injection), A. Goloborodko (immunohistochemistry and microscopy) (Figure is taken from Krishnakumar et al., 2018) 


\subsubsection{Germ plasm localization is conserved in vertebrates}

We previously demonstrated the unique shared ability of Buc and sOsk to specify germ cells in zebrafish (Figure 34) (Krishnakumar et al., 2018). To understand if the mechanism of localization is also conserved, we generated $g f p$ fusions of $b u c, s O s k$ and velo and injected RNAs of these fusions into 1-cell stage embryos. Subsequently, we performed immunostaining at 3-4 hpf to find out the colocalization of the GFP fusions with endogenous germ plasm (Figure $35 \mathrm{~A}$ ). To distinguish between the injected GFP fusions and endogenous germ plasm, we used previously described anti-Buc antibody (Riemer et al., 2015). We found that this antibody specifically recognized Buc and not Velo, sOsk or GFP in a western blot with in-vitro translated proteins (Figure 37). Therefore we concluded that it would be possible to distinguish endogenous Buc from injected Velo, sOsk or GFP alone in the co-localization assay.

To outline the architecture of germ cells we labeled membrane (beta-catenin) and the nucleus (DAPI). Buc-GFP co-localized with endogenous germ plasm (Figure $35 \mathrm{C}-\mathrm{D})$ and showed similar localization as vasa and nanos RNAs (Eno et. al., 2019; Knaut et al., 2000; Köprunner et al., 2001). Therefore, we could use the co-localization assay to study germ plasm localization in vivo. To test whether localization mechanism is conserved in vertebrates we overexpressed Velo and indeed it also localized to endogenous germ plasm (Figure $35 \mathrm{E}-\mathrm{F})$. By contrast, injected sOsk formed speckles in the nucleus (Figure $36 \mathrm{~A}-\mathrm{B}$ ), as it was shown in tissue culture cells and Drosophila PGCs (Jeske $e t$ al., 2017; Kistler et al., 2018). In contrast, GFP alone localized to membrane and nucleus, but still not to endogenous germ plasm (Figure $36 \mathrm{C}$-D). We concluded that Buc and Velo share the localization mechanism to zebrafish germ plasm, whereas sOsk does not.

In summary, the PGC induction ability of Buc, sOsk and Velo is shared in vertebrates. Furthermore, only Velo and Buc share the germ plasm localization mechanism in vertebrates, whereas sOsk localization is mediated in a different way. 
A

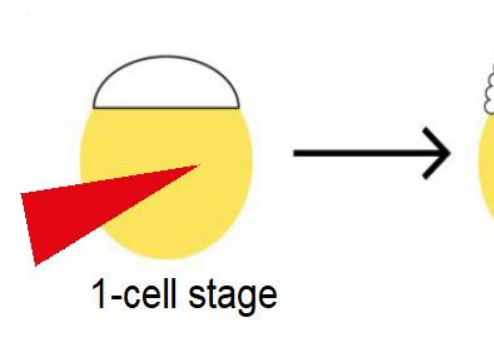

B

GFP co-localization with endogenous Buc [\%]

$\mathbf{2 0}$

40

60

8010

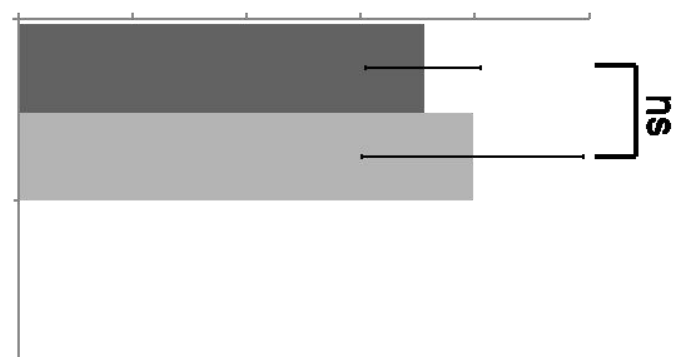

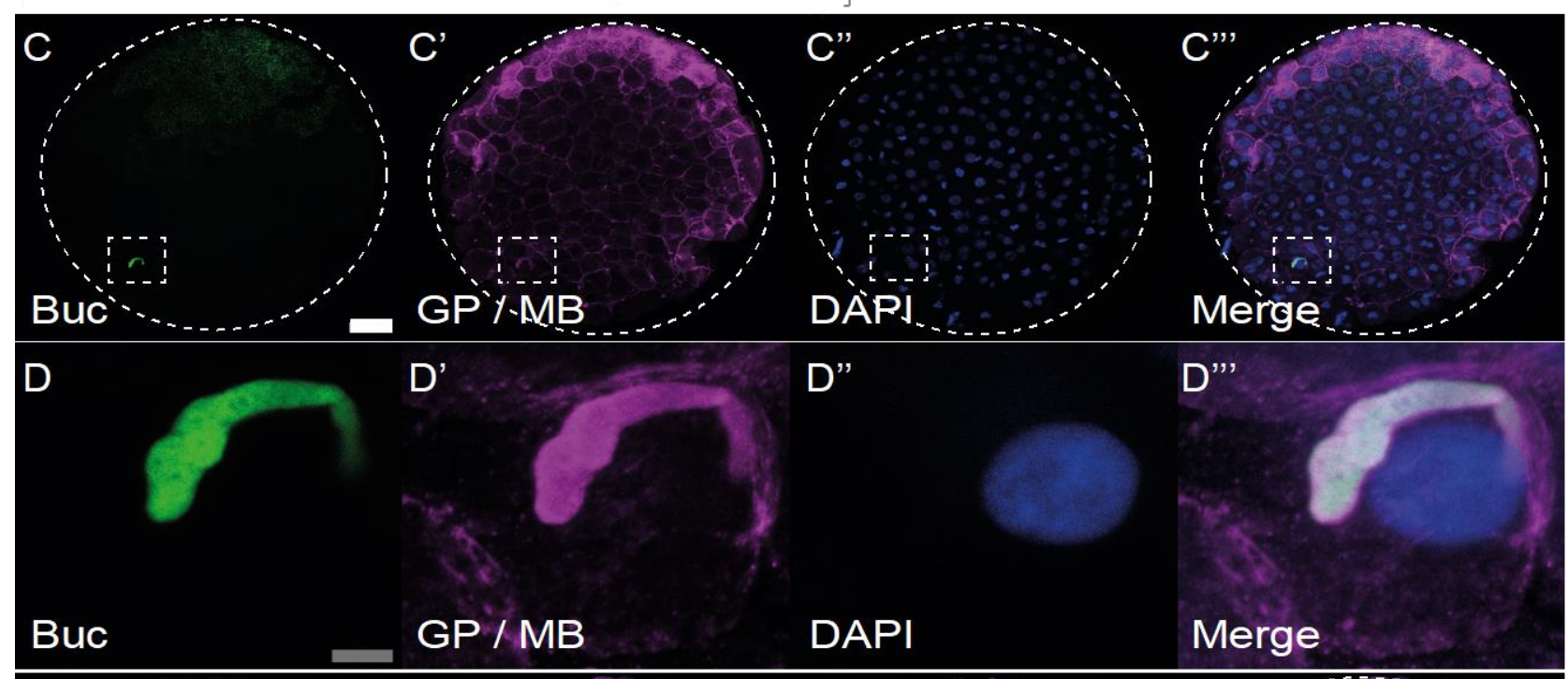

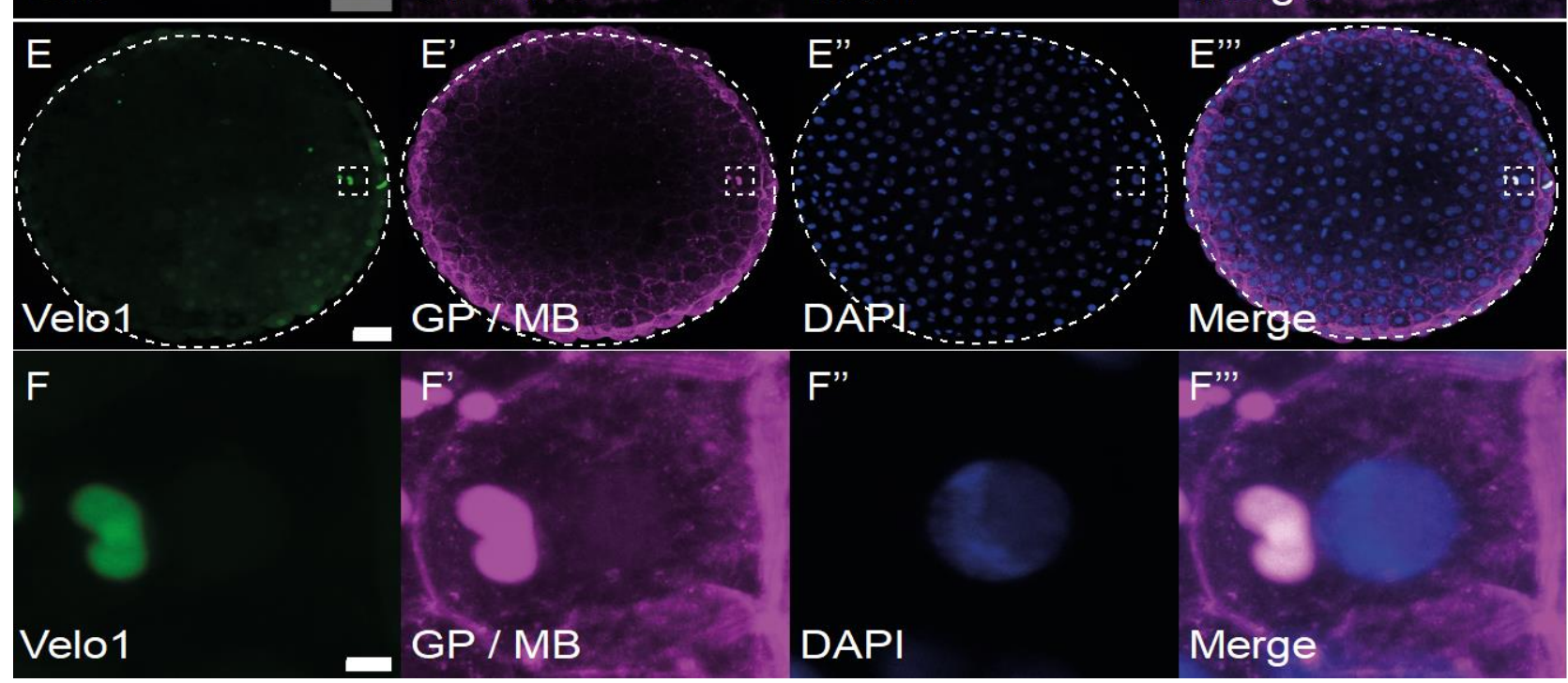

Figure 35 : Germ plasm localization is conserved in vertebrates. (A) Scheme of co-localization assay. RNA encoding GFP fusions of germ plasm organizers Bucky ball (Buc), Velo and short Oskar (sOsk) were injected into 1-cell stage embryos and scored for localization with endogenous Buc (green dots) by immunohistochemistry. (B) Quantification of co-localization assay. GFP fusions of Buc $(71 \pm 10.1 \%, \mathrm{n}=33(13,13,7))$ and Velo1 $(79.7 \pm 19.5 \% \mathrm{n}=41(15,14,5,4,3)$ $\mathrm{p}=0.6)$, but not sOsk $(0 \%, \mathrm{n}=25(5,7,13)$ or GFP alone $(0 \%, \mathrm{n}=32(18,9,5)$ show co-localization with endogenous Buc. Two tailed student's T-test, Velo-GFP vs Buc-GFP. Mean \pm standard deviation is shown. n.s: not significant. (C-K) Animal view of embryos at 3-4 hours post fertilization (hpf). Dotted circle outlines the embryo. Dotted rectangle shows magnified cell in the panel below. Co-localization of GFP with endogenous Buc was determined by immunohistochemistry: $1^{\text {st }}$ column - injected GFP fusion (green), $2^{\text {nd }}$ column - endogenous germ plasm (GP, magenta) and membrane (MB, magenta), $3^{\text {rd }}$ column - DAPI (blue) and 4th column - merge. Buc-GFP (C-C“" ", D-D“" ) or Xenopus Velo (E-E',, F-F',') co-localize with endogenous germ plasm. Scale bars (C, E): $50 \mu \mathrm{m}$ (D, F): $5 \mu \mathrm{m}$. 


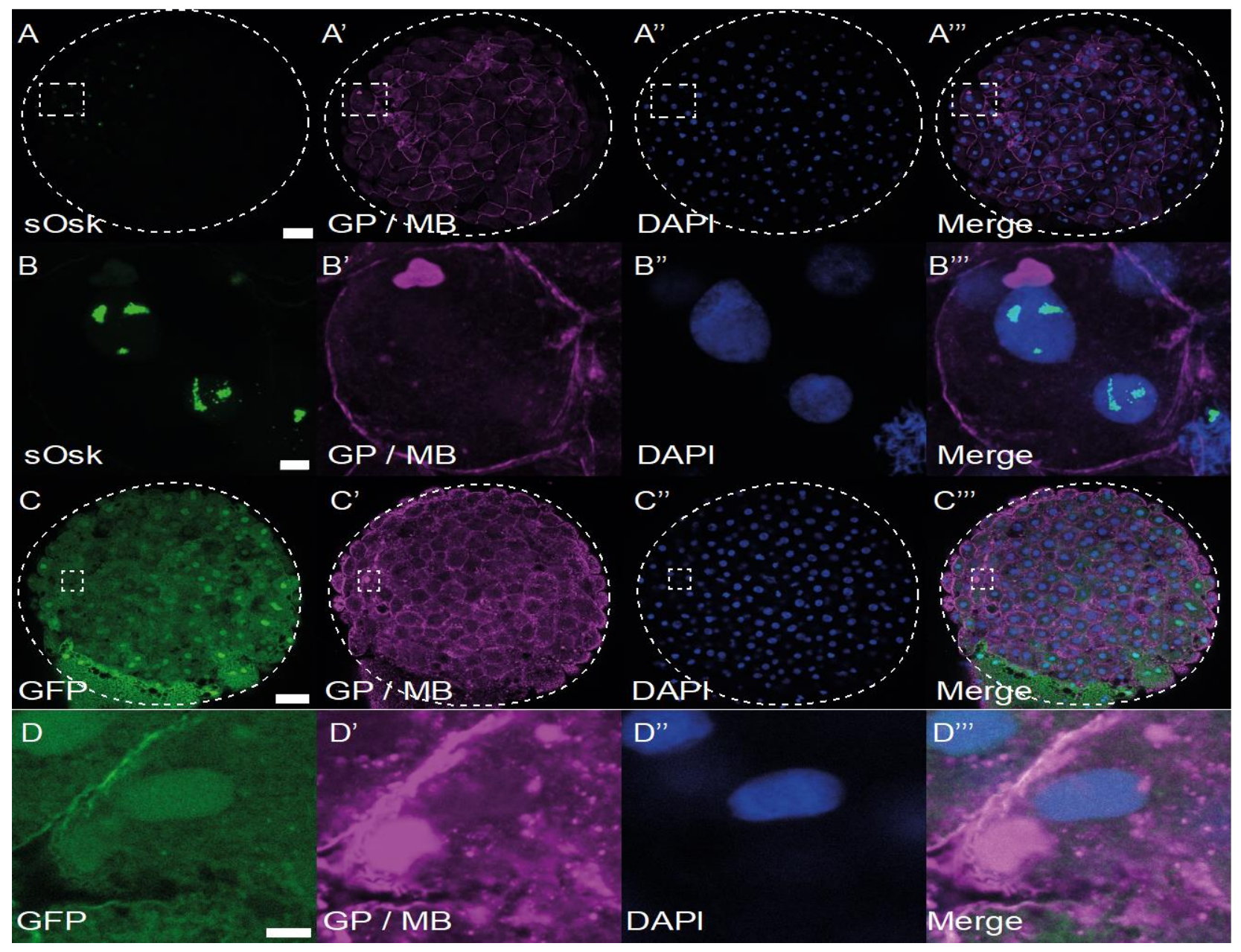

Figure 36: Germ plasm localization is conserved in vertebrates. (A-D) Animal view of embryos at 3-4 hours post fertilization (hpf). Dotted circle outlines the embryo. Dotted rectangle shows magnified cell in the panel below. Colocalization of GFP with endogenous Buc was determined by immunohistochemistry: $1^{\text {st }}$ column - injected GFP fusion (green), $2^{\text {nd }}$ column - endogenous germ plasm (GP, magenta) and membrane (MB, magenta), $3^{\text {rd }}$ column - DAPI (blue) and $4^{\text {th }}$ column - merge. Drosophila sOsk (A-A,", B-B"', ) shows nuclear localization. The GFP control (C-C"“" D-D“" $)$ shows ubiquitous low level fluorescence, after increasing brightness with Adobe Photoshop. Scale bars (A, C): $50 \mu \mathrm{m}$ (B, D): $5 \mu \mathrm{m}$. 


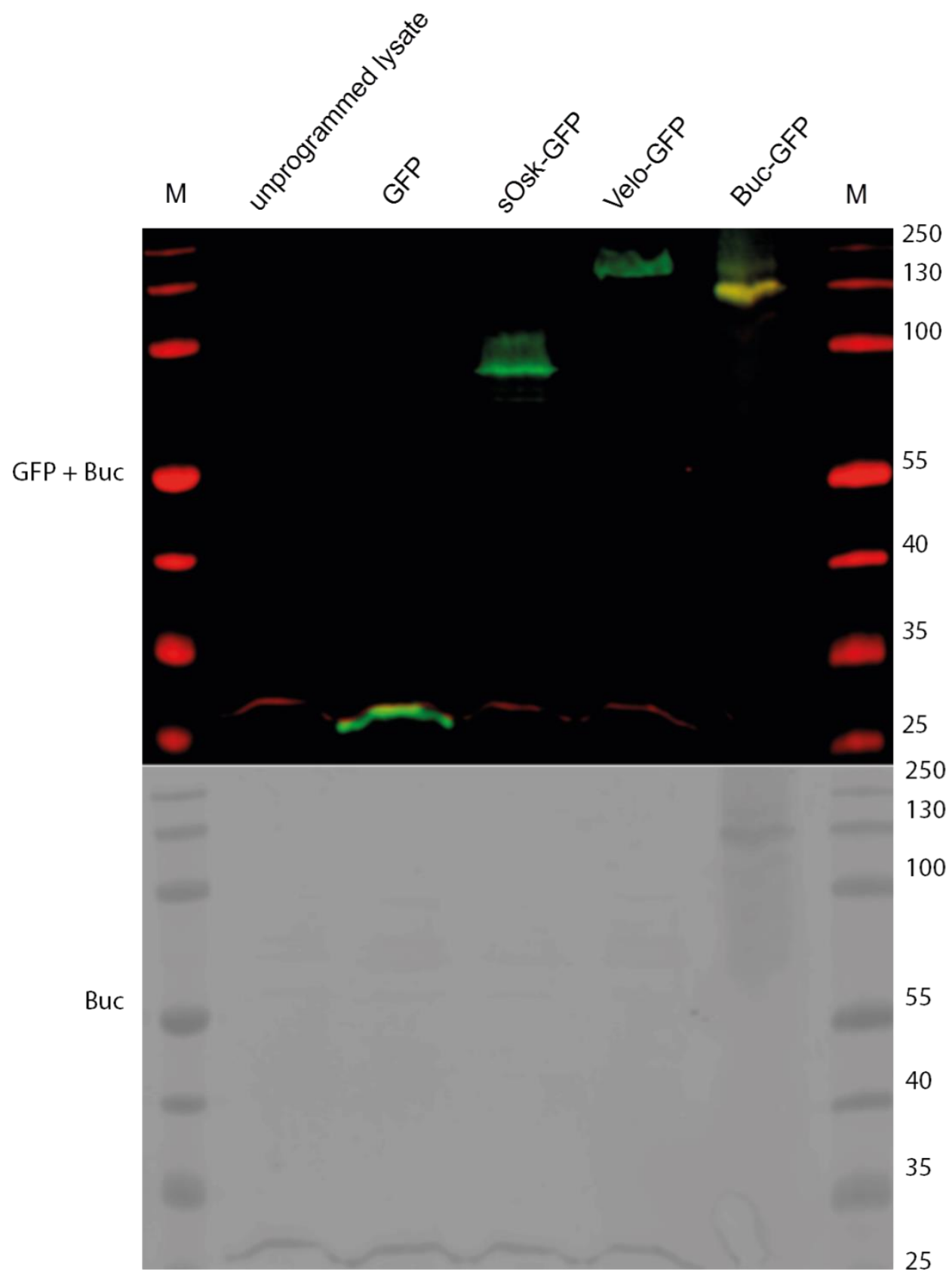

Figure 37: Germ plasm localization is conserved in vertebrates. Western blot showing anti-Buc (red in upper panel and black in lower panel) and anti-GFP (green in upper panel, absent in lower panel) antibody staining of in vitro translated proteins: Buc-GFP (lane 6), Velo-GFP (lane 5), sOsk-GFP (lane 4), GFP (lane 3). Unprogrammed lysate (lane 2) was used as a negative control for protein translation. Buc-GFP is visualized by both anti-Buc and anti-GFP antibodies (yellow in merged panel and black in lower panel), whereas Velo-GFP, sOsk-GFP and GFP alone are only recognized by anti-GFP antibody and not by anti-Buc antibody. 


\section{Discussion}

Buc was identified as the first vertebrate protein necessary for germ plasm formation and sufficient for induction of PGCs (Bontems et al., 2009). Furthermore, localization of Buc during early embryogenesis is critical event in PGC development (Hashimoto et al., 2004). Therefore, the aim of this study is to understand the molecular mechanism of germ plasm localization. In this study, we identified tight junction responsible for anchoring of Buc to cytoskeleton. Furthermore, we showed that phosphorylation of $\mathrm{ZO} 1$ is necessary for Buc degradation. Also, we showed that Buc IDRs and PrDs are not required for germ plasm localization. Our results also demonstrate that BucLoc might be hyperphosphorylated at tyrosines and this leads to degradation of Buc during embryogenesis. We used BucLoc as a bait in a biochemical screen to identify Buc interactors involved in germ plasm localization. This analysis revealed Intracellular hyaluronanbinding protein 4 - a promising candidate for linking germ plasm to cytoskeleton. Finally, we showed that the localization and PGC inducing abilities of Buc in zebrafish are conserved among vertebrates such as Xenopus but not among invertebrates such as Drosophila.

\subsection{Functional relevance of Buc co-localization with cellular structure markers}

The discovery of the four germ plasm spots has not lead so far to identification of the cellular structure that anchors Buc to cytoskeleton (Yoon et al. 1997). We showed that Buc co-localizes with various cellular structure markers during oogenesis. In contrast, Buc only co-localizes with tight junction proteins like ZO1 during embryogenesis.

\subsubsection{The Balbiani body as a storage compartment for maternally provided cellular structure markers}

We found that $\mathrm{Bb}$ contains maternally provided proteins of tight and adherens junctions and hemidesmosomes (Figure 7). Previously, it was shown that cadherins and catenins are enriched at the cortex of zebrafish oocytes, at the sites of oocyte-follicle cell contacts, indicating that unknown heterotypic tight junctions might be present between these cells (Cerdà et al., 1999). Also in Xenopus, overexpressed tight junction markers like clauding localize to the membrane of oocytes, where they probably play a role in oocyte adhesion (Vitzthum et al., 2019), whereas other tight junction components like symplekin localize to "Cajal bodies" during Xenopus oogenesis (Hofmann et al., 2002). Therefore, our finding of cellular structure markers localization to the $\mathrm{Bb}$ is unique. Interestingly, immunostaining of $\mathrm{ZO} 1$ and E-cadherin in the Buc ${ }^{\mathrm{p} 106}$ mutant background, where the $\mathrm{Bb}$ is not assembled, showed distribution of these cellular structure markers over the entire cytoplasm of oocytes (data not shown). This result suggests that ZO1 and E-cadherin do not act upstream by localizing Buc to $\mathrm{Bb}$ during oogenesis. Therefore, we assumed that none of the detected cellular structure markers is functional in $\mathrm{Bb}$. This is in line with previous views of $\mathrm{Bb}$ as a storage hub for RNAs and healthy mitochondria during development in order to pass them into the PGCs (Cox, 2003; Kloc et al., 2004; Kogo et al., 2011; Marinos \& Billett, 1981). Therefore, we think that zebrafish Bb stores and protects cellular structure markers in order to pass them on to the embryo.

Previously, it was shown that Buc homologue in Xenopus Velo1 creates amyloid-like matrix in Bb, entrapping organelles like mitochondria (Boke et al., 2016). We also demonstrated that Buc forms preferentially stable aggregates in Bb during oogenesis (Figure 19, Figure 20). Hence, we think that 
Buc also could form similar protein networks, which can entrap organelles and proteins such as different cellular structure markers. However, it still needs to be addressed whether the localization of cellular structure markers is mediated by direct protein-protein interactions with Buc or by their entrapment in a protein matrix formed by Buc.

\subsubsection{Buc co-localizes with tight junction proteins during early embryogenesis.}

During late stage oogenesis $\mathrm{Bb}$ disassembles and during early embryogenesis we no longer found colocalization of adherens junctions and hemidesmosomes with germ plasm. Fascinatingly, tight junction markers remained co-localized with germ plasm during early embryogenesis (Figure 8). Interestingly, at the 4-cell stage, $\mathrm{ZO} 1$ and Buc co-localize to one domain at the cleavage furrow (Figure $8 \mathrm{C}, \mathrm{D})$. In contrast, at the 8-cell stage $\mathrm{ZO} 1$ localizes to the two separate domains: along the entire length of the first two cleavage furrows and to the germ plasm (Figure $8 \mathrm{~A}, \mathrm{~B}$ ). Our result also show that germ plasm co-localizes with ZO1-A, whereas most likely ZO1-B localizes to the entire length of the furrows. Hence, we assume that a segregation of ZO1-A from ZO-B takes place at the 4- to 8-cell transition during germ plasm compaction (Lindeman \& Pelegri, 2010). The segregation of both ZO1 isoforms might be crucial for germ plasm localization. We hypothesize that the localization of ZO1-A at the distal ends of the cleavage furrow contributes to formation of a tight junction which anchors germ plasm to cytoskeleton. The presence of tight junction in zebrafish was confirmed as late as at the onset of epiboly by transmission electron microscopy. At this stage tight junctions are visible in the enveloping layer as typical electron dense plaques at the luminal end of the lateral membrane of adjoining cells (Kimmel et al., 1995). However, it remains to be addressed if maturated tight junctions are present within germ plasm at the 8-cell stage and whether there is a difference between the germline and somatic tight junctions. Addressing these questions will help to further understand the molecular mechanisms of germ plasm localization.

Different ZO1 isoforms were also studied in mouse tight junction assembly (Furuse et al., 1993, 1994). It was demonstrated that the final step in tight junction formation is mediated by the $\mathrm{ZO} 1 \alpha^{+}$ isoform. The interaction of $\mathrm{ZO} 1 \alpha^{+}$with occludin is crucial for the assembly of both proteins at the membrane and finalizing the tight junction formation. In analogy to the mouse $\mathrm{ZO} 1 \alpha^{+}$, the germline $\mathrm{ZO} 1$ isoform in zebrafish might also require another tight junction component like occludin to localize and form a distinct type of tight junction in germ cells. However, we could not detect presence of occludin in the $\mathrm{Bb}$ using an anti-mouse claudin antibody (data not shown). Using different antibodies or immunohistochemistry approaches for occludin detection will be a focus of future research.

Other tight junction components which can contribute to understanding of germ plasm localization mechanism are claudins. Claudins have important function in formation of tight junction permeability barrier and the role in linking of claudins to the underlying cytoskeleton (Anderson et al., 2004; Tsukita \& Furuse, 2000). Also it was shown that Xenopus claudin (Xcla) is critical for ZO1 localization (Brizuela et al., 2001). Interestingly, previously claudin D was identified in the biochemical screen for Buc localization signal interactors (Riemer 2014, Doctoral dissertation). However, studying this or other claudin genes are difficult due to lack of antibodies and a high number of claudin genes present in teleosts (Loh et. al., 2004). Interestingly, Buc and ZO2 did not co-localize (Figure $12 \mathrm{C}, \mathrm{D})$. Therefore, we assume that $\mathrm{ZO} 2$ does not play a role in germ plasm localization.

Next to the co-localization of Buc with ZO1 at the 8-cell stage, we found similar results at the 512cell stage. Also ZO1 co-localizes with Buc in PGCs at $1 \mathrm{dpf}$ (Rostam, unpublished). Possibly, at 1 
dpf we observe ZO1 made after the genome transcription activation. However, the function of the continuous co-localization of Buc with ZO1 is unclear.

\subsubsection{The role of E-cadherin in germ plasm compaction}

At the 8-cell stage, Buc and E-cadherin localize to the two separate domains at the furrow (Figure 9). E-cadherin localized along the proximal ends of the $1^{\text {st }}$ and $2^{\text {nd }}$ furrows (Figure 9 B'), whereas Buc localized to the distal ends (Figure 9 A'). It has been shown that in Xenopus, maternal cadherins are active from the onset of embryogenesis and are involved in intercellular adhesion (Fleming et al., 2000). Also, it was shown that intracellular calcium regulates cell-cell adhesion in fibroblast through remodeling of cortical actin and recruitment of cadherins into intercellular junctions (Ko et al., 2001). Furthermore, it was shown that inhibition of calcium release or downstream mediators like calmodulin during first embryonic cleavages in zebrafish inhibits germ plasm compaction (Eno et al., 2018a). Remarkably the levels of p-NMII remain normal upon defects in germ plasm compaction in nebel mutants or inhibition of calcium release in WT embryos (Nair et al., 2013). Since the p-NMII is involved in germ plasm compaction (Urven et al., 2006), it is tempting to speculate that the depletion of intracellular calcium affects some of the upstream signaling that acts on NMII. It was shown that E-cadherin can activate RhoA, which in its turn activates NMII by phosphorylation (Watanabe et al., 2009). It is also believed that germ plasm compaction is mediated via RhoA/ROCK/p-NMII pathway (Miranda-Rodríguez et al., 2017). Therefore, we think that maternal cadherins can be one of these upstream proteins which induce germ plasm compaction via p-NMII. However, we do not exclude a possibility that NMII itself contributes to activation of adherens junctions assembly via the cadherins. It was shown that NMII can act via actin to bring adherens junction components in close proximity and by this facilitate their function (Vicente-Manzanares et al., 2009). Overall, this leads to a hypothesis that activation of intracellular calcium signaling leads to E-cadherin localization and expansion along the cleavage furrows (Figure 38). Then, E-cadherin activates RhoA, which induces germ plasm compaction via phosphorylation of NMII by ROCK. 


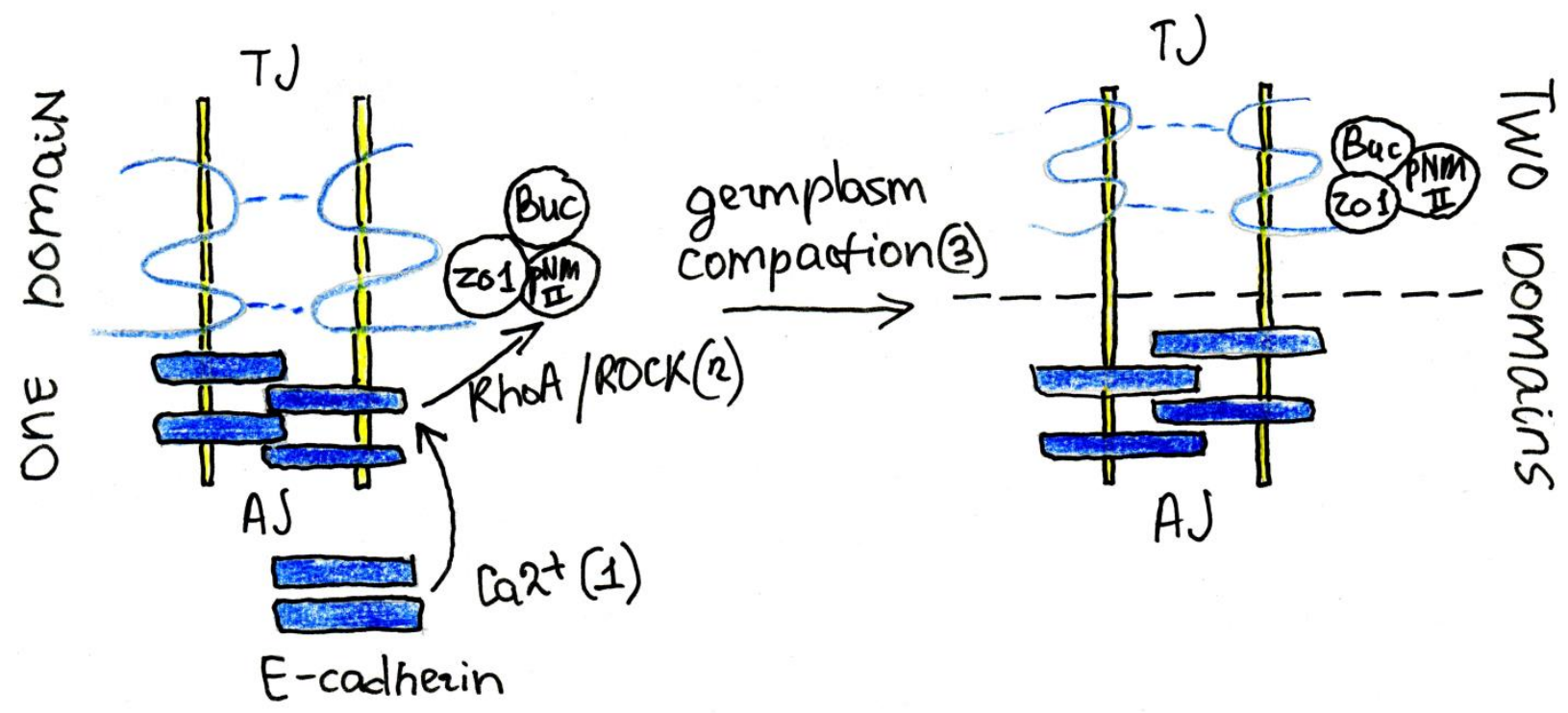

Figure 38: A hypothetical model for involvement of adherens junctions in germ plasm compaction. Schematic drawings of cleavage furrows before (left) and after germ plasm compaction (right). (Before germ plasm compaction) Adherens junction component E-cadherin and tight junction component ZO1 localize to the same domain at the cleavage furrow as germ plasm. Intracellular calcium (1) activates assembly of E-cadherin at the membrane, whereas activated Ecadherins induces activation of the RhoA/ROCK signaling. Subsequently, ROCK activates non-muscle myosin II (NMII) by phosphorylation of the regulatory light chain (2). (During germ plasm compaction) Then p-NMII moves germ plasm, together with ZO1 to the distal ends of the cleavage furrows. By this, the localization domains of E-cadherin and germ plasm/ZO1/p-NMII are separated at the cleavage furrow.

\subsubsection{Functionality of Buc and Z01 co-localization}

To address the role of $\mathrm{ZO} 1$ in germ plasm localization, we induces $\mathrm{ZO} 1$ dephosphorylation using the PKC inhibitor CC. Surprisingly, we found that CC inhibits Buc degradation (Figure 15, Figure 16, Figure 17). However, $\mathrm{CC}$ did not disrupt Buc localization to the four spots.

We found that Buc levels were elevated upon $\mathrm{CC}$ treatment already at the 8-cell stage (Figure $17 \mathrm{~A}$, $\underline{B}$. Recently it was proved that ZO1 undergoes phase separation to form membrane attached compartments. By this, ZO1 enriches and localizes ZO1-interacting proteins to a membrane (Beutel et al., 2019). Furthermore, previously it was demonstrated that dephosphorylated ZO1 efficiently undergoes phase separation into liquid droplets (Beutel et al., 2019). Hence, the elevated Buc levels at the cleavage furrows can be due to an enhanced phase separation behavior of ZO1. Our data also suggests that the enhanced phase separation behavior of ZO1 stabilized and protected unlocalized cortical Buc granules from degradation (Figure $17 \mathrm{~A}, \mathrm{~B}$ ). This is in line with recent publication that shows a decline of PGL granule proteins degradation in C.elegans upon their accelerated phase separation behavior (Zhang et al., 2018).

We also observed much higher induction of unlocalized Buc-GFP levels with $5 \mu \mathrm{M}$ CC. Interestingly, $5 \mu \mathrm{M}$ CC inhibits myosin light chain kinase (MLCK) and myosin reorganization in vitro (Strassheim et al., 1999). Nevertheless, even the $5 \mu \mathrm{M}$ CC treatment did not affect the number of localized BucGFP spots (Figure 16 C). Previously, it was shown that inhibition of MLCK by the drug h1152 results in germ plasm compaction defects at the 4-cell stage in zebrafish (Miranda-Rodríguez et al., 2017). Previously, it was also shown that MLCK inhibition stabilizes ZO1 at tight junctions in mice (Yu et 
al., 2010). Our results also show that inhibition of PKC and MLCK by the $5 \mu \mathrm{M} \mathrm{CC}$ stabilizes ZO1 at the cleavage furrows in zebrafish. However, we did not observe any germ plasm compaction defects, as it is the case with the h1152 MLCK inhibitor. Thus, our data suggests the phosphorylation of $\mathrm{ZO} 1$ by the PKC is upstream of the MLCK activation.

It was demonstrated that CC inhibits de novo tight junction biogenesis (Stuart and Nigam, 1995). In contrast, to the de novo tight junction biogenesis, Buc and ZO1 already co-localize during oogenesis and therefore probably assemble into a protein complex before the onset of embryogenesis. Therefore, there is no de novo assembly of Buc at the tight junctions which can be inhibited by CC. An alternative approach to show that tight junction anchors Buc to cytoskeleton might be to inhibit phase separation behavior of ZO1. We expect that this will lead to a decrease in Buc and ZO1 localization to the cleavage furrows. Hence, a further study will focus on applying chemical compounds which activate PKC such as Okadaic Acid, PMA or Bryostatin 1.

The results of the ZO1 dephosphorylation on germ plasm localization lead to a hypothetical model where PKC phosphorylation of $\mathrm{ZO1}$ is required for the tight regulation of localized and unlocalized Buc levels (Figure 39). Possibly, a dephosphorylated ZO1 scaffolds Buc more efficiently. Subsequently, this leads to the observed enhanced accumulation Buc and ZO1 at cleavage furrows.

To sum up, Bb stores cellular structure components, followed by their deposition at the forming cell membranes during embryogenesis. Fascinatingly, only tight junction marker ZO1 remains colocalized with germ plasm during early embryogenesis. This is the first evidence that ZO1 colocalizes with germ plasm. We also show for the first time the presence of two different maternal $\mathrm{ZO} 1$ isoforms. The germline specific ZO1 probably defines which cells will become germ cells by anchoring and stabilizing germ plasm early during development. In contrast, the somatic ZO1 might function as part of regular tight junctions in the most apical embryonic cells. So far, we think ZO1 interacts with Buc and by this regulates Buc degradation. Future research will focus on using PKC activators to show whether ZO1 also anchors Buc to cytoskeleton. 

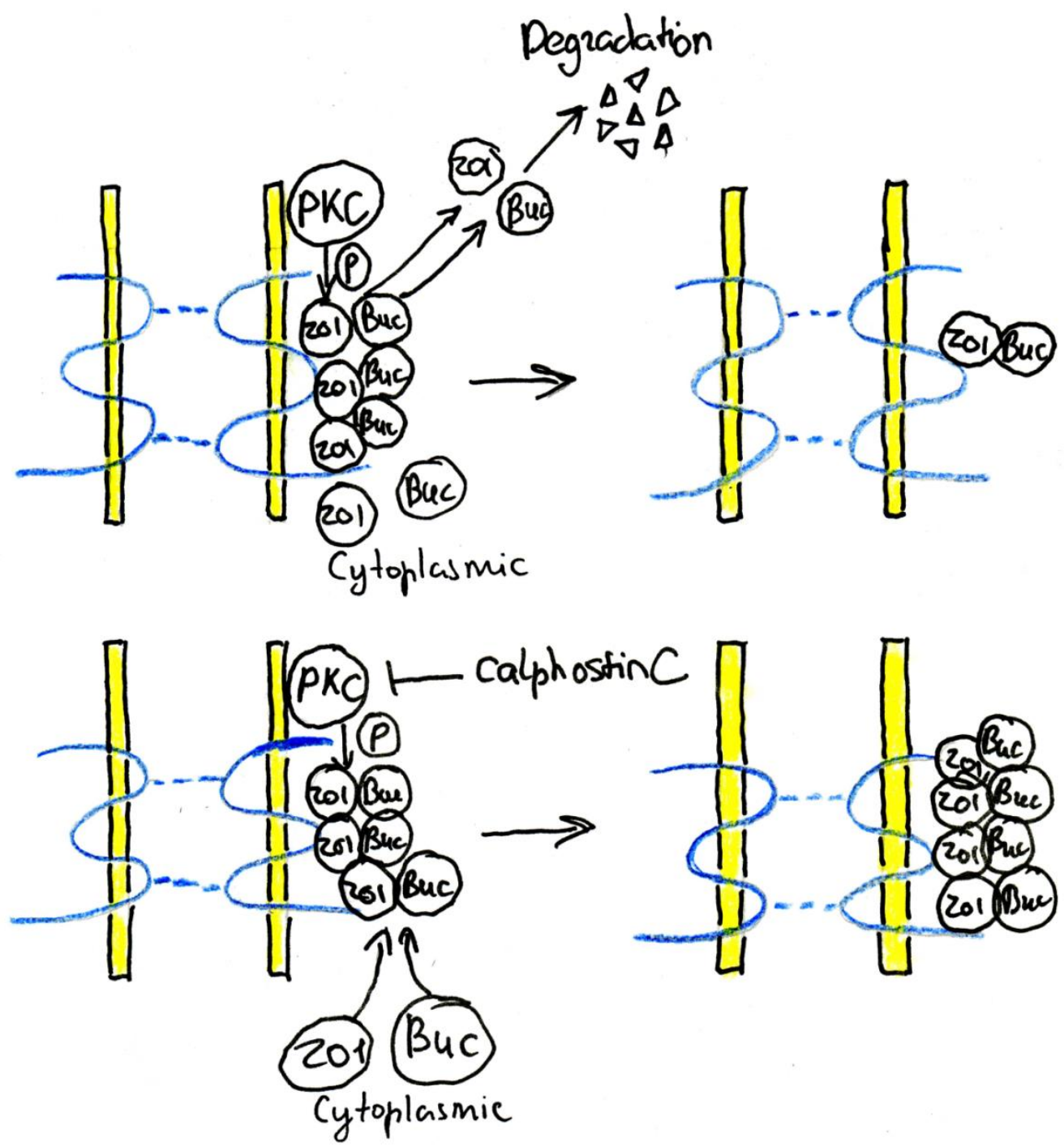

Figure 39: A hypothetical model: ZO1 phosphorylation is required for Buc degradation. (Upper drawing) Protein Kinase C (PKC) phosphorylates ZO1 at the furrows, leading to degradation of some fraction of the localized ZO1 and Buc. (Lower drawing) Calphostin $\mathrm{C}$ inhibits PKC leading to reduced phosphorylation of ZO1. This leads to elevated levels of Buc and ZO1 at the cleavage furrows and in cytoplasm. This leads also to the recruitment of more cytoplasmic $\mathrm{ZO} 1$ and Buc to the cleavage furrows.

\subsection{Bucky ball plays a role in germ plasm aggregation and localization}

Buc is the first protein in vertebrates required for germ plasm assembly and localization (Bontems $e t$ $a l ., 2009)$. However, prior to this study it was unclear whether Buc IDRs and PrDs play a role in germ plasm localization. Furthermore, it was unclear which protein domains in Buc play a role in germ plasm localization vs aggregation.

3.2.1 PrDs in the BUVE motif of Buc might be responsible for germ plasm aggregation during oogenesis

It was demonstrated that the $\mathrm{N}$-terminal domain of Velo1 forms insoluble amyloids during Xenopus oogenesis via PrDs (Boke et al., 2016). We demonstrated that PrDs in Buc are not required for germ plasm localization (Figure 22, Figure 25). Hence, PrDs in the BUVE motif of Buc might also be 
responsible for aggregation of the zebrafish $\mathrm{Bb}$. However, using the PLAAC software to predict the protein aggregation propensity, we found 8 times lower aggregation propensity values (PAPA values) for Buc than for Velo1 (0.04 vs 0.33). Moreover, sOsk which does not contain predicted PrDs has a higher predicted PAPA value than Buc (0.06 vs 0.04). Hence, based on the PLAAC software prediction, the PrDs in Buc are not so potent to form amyloid aggregates compared to Velo1. However, despite the poor amyloid propensity prediction, solid aggregates of Buc are present in the $\mathrm{Bb}$ (Figure 19, Figure 20). The poor prediction of Buc to form amyloids is probably equalized by a tight regulation of Buc aggregation at post-translational or protein-protein interaction levels. Previously it was demonstrated that intracellular condensate formation is mediated by phosphorylation, methylation or ADP ribosylation (Alberti, 2017) Recent experimental evidences show indeed that regulation of Buc aggregation behavior by interaction of Buc dimethylated arginines (DiMeArgs) (aa 630, 629) with Tdrd6 (Roovers et al., 2018).

To investigate other possible post translation modifications involved in Buc aggregation, we checked phosphorylation status of $\mathrm{Bb}$. We found strong enrichment of phosphotyrosines in $\mathrm{Bb}$ of the late stage IB oocytes (Figure 31). Hence, it is possible that Buc aggregation is regulated via phosphorylation. This is in line with recent findings of IDPs clearance induced by multi-site phosphorylation (Carpenter et al., 2018). Since we found a solid and a less solid Buc fractions in Bb, it is tempting to speculate that phosphorylation of the solid fraction of Buc during late stage embryogenesis induces its clearance and releases the less solid Buc fraction to form germ plasm during embryogenesis. However, firstly it needs to be addressed whether Buc is phosphorylated. Therefore, it remains unclear whether post-translational modifications of Buc play a role in zebrafish Bb formation.

To sum up, the PrDs within the localization signal of Buc are not required for germ plasm localization. We assume that these PrDs might be involved in Bb aggregation during oogenesis, as it was shown for Velo1 (Boke et al., 2016).

\subsubsection{Buc aggregates via its IDRs during embryogenesis}

We discovered that the most of Buc protein sequence carries intrinsic disorder (Figure $18 \mathrm{~A}$ ). However, the first 100 aa of Buc do not contain IDRs. Hence, IDRs do not play a role in germ plasm localization. We also showed that IDRs alone are able to aggregate in vitro (Figure 21 D-D'). Therefore, we assume that IDRs are involved in aggregation of germ plasm. This assumption is in line with hypothesis that IDRs drive phase separation of proteins and by this can form liquid-like or more solid aggregates (Kato et al., 2012; Kroschwald et al., 2015). However, Buc IDRs alone do not drive germ plasm aggregation in-vivo. The construct lacking the N-terminal region (DeltaBuc11-88GFP) shows ubiquitous level of fluorescence upon overexpression in zebrafish embryos (Riemer 2014, Doctoral dissertation). Hence, it can be hypothesized that the IDRs of Buc need to be first localized in order to become aggregated and form a hydrogel. Probably, the presence of RNA or other proteins within germ plasm granules is required for the localized formation of Buc hydrogels. This is in agreement with studies showing that low-complexity regions of IDPs can accommodate heterotypic polymerization (Han et al., 2012; Kato et al., 2012).

In summary, we think that Buc localization is required to aggregate germ plasm during embryogenesis via IDRs of Buc. 


\subsubsection{The prion-like domains of Buc are not required for germ plasm localization}

We demonstrated that PrDs in Buc are not required for germ plasm localization (Figure 22, Figure 25). However, our results regarding the requirement of the C-terminal PrD for germ plasm localization carry a degree of contradiction. On the one hand we found that both BucLoc deletions of the half of the C-terminal PrD ( $\Delta 62-66$ and $\Delta 67-71)$ localize as the WT BucLoc (Figure 27). On the other hand, we found decreased levels of expression for the full deletion $(\Delta 64-71)$ or mutant $(3 \mathrm{D})$ of the C-terminal PrD of BucLoc, while the BucLoc 3D mutant still weakly localized to endogenous germ plasm (Figure 24). The reduced expression levels of these BucLoc constructs can be caused either by induced defects at the protein structural level or by removal of a regulatory sequence within the predicted $\operatorname{PrD}$ sequence.

Remarkably, both the $\Delta 62-66$ and $\Delta 67-71$ BucLoc constructs have a deletion of one out of 2 tyrosines present in the C-terminal $\operatorname{PrD}$, whereas the full deletion of the second prion domain $(\Delta 64-71)$ represents a deletion of two tyrosines. Therefore, we hypothesized that the phosphorylation of both tyrosines might be an important regulatory mechanism in localizing Buc or regulating its degradation. Our hypothesis, is in line with previous results of phosphorylation-mediated degradation of the germ plasm organizer Oskar during Drosophila embryogenesis (Morais-de-Sá et al., 2013). This hypothesis, is further confirmed by our biochemical and immunohistochemistry experiments showing that germ plasm and BucLoc domain are not phosphorylated during embryogenesis (Figure 30, Figure 31). Therefore, our data implies that a function of the PrD within BucLoc might be to regulate germ plasm localization and/or degradation via tyrosine phosphorylation.

To sum up, our results can be summarized in the following model (Figure 40). BucLoc (aa 31-78) is required for germ plasm localization, whereas the IDR is required for germ plasm aggregation. Moreover, we think that Buc localization is the primary event in the establishment of germ plasm, followed by germ plasm aggregation via Buc IDRs. Additionally, germ plasm localization and/or degradation might be regulated by tyrosine hyperphosphorylation of the BucLoc domain. 


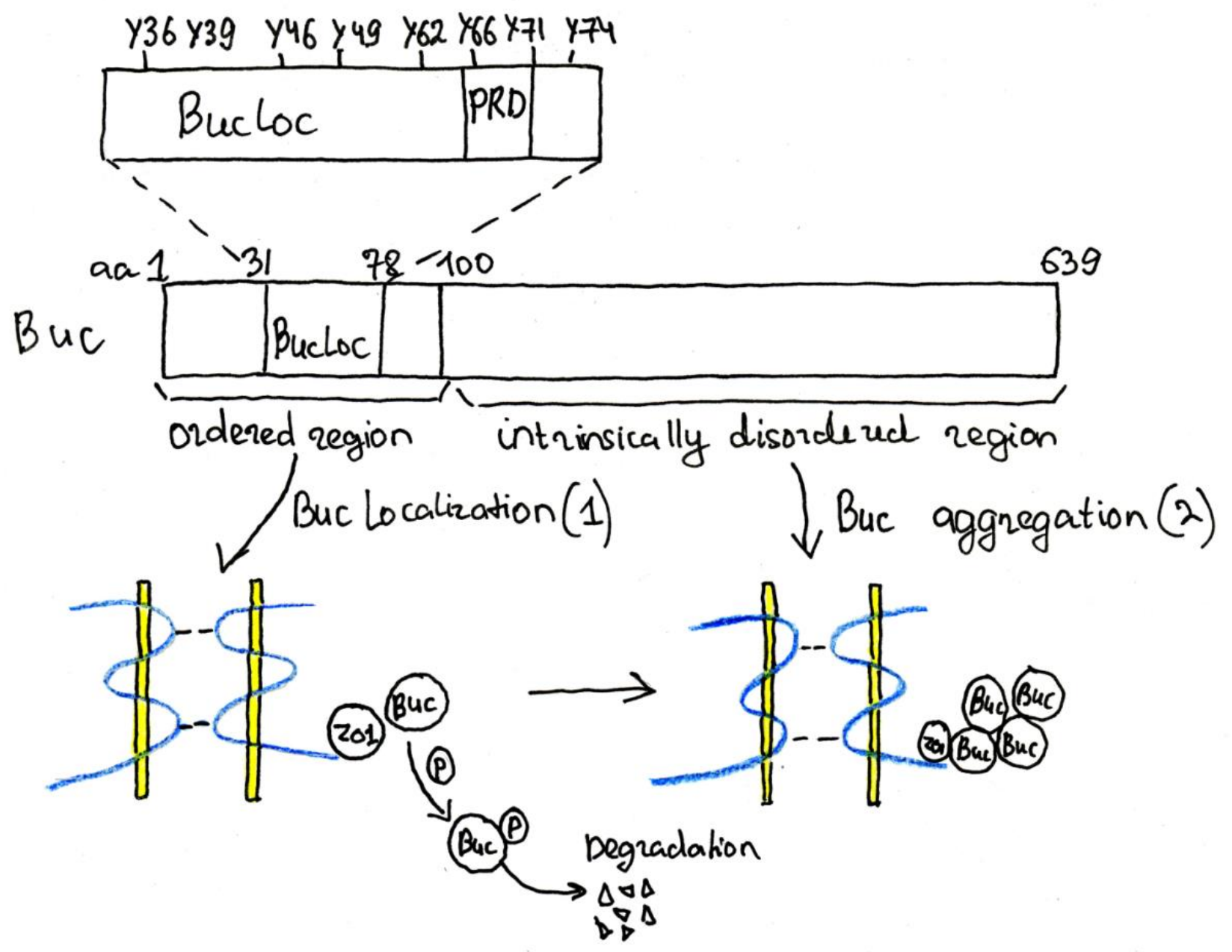

Figure 40: A hypothetical two step model of germ plasm establishment in zebrafish. Buc protein sequence (middle scheme) consists from the ordered N-terminal region (residues 1-100) and the intrinsically disordered C-terminus (100639). The ordered N-terminal region of Buc contains BucLoc domain (31-78) which is sufficient for germ plasm localization. BucLoc contains multiple tyrosines which might be phosphorylated and involved in the regulation of Buc localization and/or degradation (Upper scheme). The prion-like domain (PRD) also contains two tyrosines (Y66, Y71) which might be also important regulatory sites for Buc localization and (or degradation. (Lower scheme) We think that germ plasm localization via BucLoc interaction with $\mathrm{ZO} 1$ is the first event before the germ plasm aggregation takes place via IDRs of Buc (Plasma membrane - yellow, tight junction - blue). 


\subsection{The core BucLoc interactome}

We identified 23 proteins interacting with Buc in the MS analysis (Figure 33, Table 1). Most of the identified proteins have a function in RNA processing, protein translation and degradation. Previous studies identified motor proteins, RNA helicases, RNA regulatory proteins and glycolytic enzymes as major constituents of germ granules in Drosophila (Zheng et al., 2016; DeHaan et al., 2017). We also found RNA helicases and RNA regulatory proteins as part of the BucLoc interactome. However, we did not find any motor proteins or glycolytic enzymes in our analysis. The reason for this difference might be due to protein sample preparation for the MS analysis or a difference in germ granule composition between Drosophila and zebrafish.

Furthermore, we only identified one protein (Ihabp4) which binds to cytoskeleton and hence can play a role in anchoring germ plasm (Huang et al., 2000). Surprisingly, we did not identify any tight junction components in the main 23 BucLoc interactors. Although, ZO2 peptides were present in our raw data of 291 candidate proteins, the peptide counts were very low. Currently, we hypothesize that BucLoc interacts with $\mathrm{ZO}$ proteins via SH3-binding domains. SH3 domains interact weak and transient with other proteins (Okada et al., 2011). Hence, an effective pull-down of ZO proteins requires use of photoreactive amino acid cross-linkers (Okada et al., 2011).

We also identified two well characterized germ plasm proteins: Piwil1 and Tdrd6. In zebrafish, Tdrd6 is also identified in MS analysis with a pull-down of full length Buc (Roovers et al., 2018). The presence of Tdrd6 in the core BucLoc interactome, is probably due to BucLoc interaction with endogenous Buc. Previously we identified endogenous Buc in the pull-down and MS analysis of Buc localization signal (Riemer 2014, Doctoral dissertation). Also, MS analysis of the Bb in Xenopus shows that Velo1 is the most abundant protein there (Boke et al., 2016). However, we did not identify endogenous Buc in our MS analysis. We only identified Buc2l, a protein carrying 28\% similarity on a sequence level with Buc. Therefore, there might a mistake have occurred in annotation of Buc peptides.

Thus, the profile of the identified BucLoc interactome is similar to the main germ granule proteins found in other studies. However, our analysis lacks some of the most promising candidates involved in germ plasm localization like ZO proteins. Future studies will focus on using photoreactive amino acid cross-linkers to identify proteins which interact weakly or only transiently with BucLoc, like $\mathrm{ZO}$.

\subsection{Conserved functions of Buc}

We showed that both Buc and sOsk induce PGCs in the 16-cell assay (Figure 34). However, only Velo and Buc localize to zebrafish germ plasm, whereas sOsk is localized to the nucleus (Figure 36, Figure 35). It was shown that sOsk activates Vasa helicase activity and by this might lead to activation of germline specification programs (Jeske et al., 2017). Hence, the PGC-inducing ability of Buc and sOsk in the 16-cell assay might also depend on the activation of zebrafish Vasa helicase activity. It is remarkable, that despite the nuclear sOsk localization, it still induces formation of PGCs. Hence, probably upon sOsk overexpression in a corner blastomere, some fraction of sOsk localizes to germ plasm and induces PGC formation later on during development via activation of Vasa helicase activity. 
Our current model of germ plasm localization in zebrafish involves ZO1 mediated anchoring of Buc to cytoskeleton. Our result that Velo1 localized to zebrafish germ plasm suggests that Velo1 carries a localization sequence, which is similar to BucLoc. This result also suggests that germ plasm is also anchored via ZO1 in Xenopus. This is in line with the formation of tight junctions early during Xenopus embryogenesis (Fleming et al., 2000). In contrast to this hypothesis, we cannot exclude a possibility that endogenous Buc and Velo1 co-aggregate and this results in Buc and Velo1 colocalization in our experiment.

Our result suggests that sOsk protein does not carry a germ plasm localization signal. This hypothesis is in line with a model stating that localization of sOsk RNA is required for sOsk protein localization (Rongo et al., 1995; Ephrussi et al., 1991; Kim-Ha et al., 1991). However, it was shown that actin cytoskeleton is required to maintain localized sOsk protein during Drosophila oogenesis (Jankovics et al., 2002; Polosello et al., 2002). The septate junctions (SJ) in invertebrates are considered be analogous to tight junctions in vertebrates (Matter \& Balda, 2003). Hence, we do not exclude a possibility that SJ in Drosophila are associated with the pole plasm. Interestingly, the ZO1 homolog in Drosophila the Lethal(1)discs-large-1 (Dlg) localizes to the germline-contacting follicle cells at the posterior domain of Stage 8 and 9 egg chambers ( $\mathrm{Ng}$ et al., 2016, Willott et al., 1993). At the same stage of development sOsk protein is translated at the posterior pole of oocytes (Markussen $e t$ al., 1995). Hence, a possibility exists for sOsk interaction with Dlg protein. Dlg contains similar SH3 domains as ZO1, but lacks its C-terminal domain compared to ZO1 (Willott et al., 1993). Thus, it is tempting to speculate that the reason sOsk does not localize to zebrafish germ plasm is its preference to interact with the invertebrate $\mathrm{ZO} 1$ isoform which is structurally different from the zebrafish $\mathrm{ZO} 1$.

To sum up, vertebrates and invertebrates share ability to induce PGC formation from somatic cells. However, the molecular mechanism of germ plasm localization is only conserved in vertebrates but not in invertebrates.

\section{Conclusion}

Buc is the first protein in vertebrates required for germ plasm assembly and localization (Bontems $e t$ $a l ., 2009)$. Furthermore, Buc is an important factor in germ cells specification. Since the discovery of the four germ plasm localization spots, the molecular mechanism of germ plasm localization remained unknown.

Our results demonstrate that $\mathrm{Bb}$ stores various maternal cellular structure markers in their inactive state during early oogenesis. In contrast, during early embryogenesis only the tight junction component ZO1-A co-localizes with Buc. Hence, we assume that ZO1-A becomes functional during early embryogenesis and forms a tight junction to which germ plasm is anchored. Thus, the four germ plasm localization spots are formed due to the segregation of the germline specific ZO1-A isoform from the rest of the inherited maternal components during the compaction of germ plasm to the distal ends of cleavage furrows. To further understand how germ plasm is anchored to tight junction, the presence of mature tight junctions during early embryogenesis in zebrafish need to be addressed by electron microscopy.

We demonstrated a role of ZO1 phosphorylation in Buc degradation. Based on this result we assume that PKC-mediated phosphorylation of ZO1 is required for the tight regulation of Buc levels during early embryogenesis. Our data also supports a model where phosphorylation status of ZO1 determines its ability to anchor Buc at the furrow. So far, inhibiting ZO1 phosphorylation resulted in an enhanced 
Buc localization at the furrow. Future research will focus on inducing ZO1 phosphorylation (e.g. by a PKC activator) to create a loss of function phenotype, where Buc is not localized to the furrow. Also, the nature of Buc and ZO1 interaction need to be investigated as well. These experiments are essential in further understanding how Buc is anchored by ZO1 at the furrow.

Mapping of BucLoc domain showed that Buc PrDs are not required for germ plasm localization during embryogenesis. Furthermore, we showed that BucLoc domain does not contain predicted IDRs. Hence, we assume that IDRs are not involved in germ plasm localization. In contrast, our data suggests that the aggregation of germ plasm during embryogenesis depends on Buc IDRs. Since, IDRs alone lack the ability to form aggregates in vivo, we assume that the localization of Buc is the critical step in inducing germ plasm aggregation via the IDRs during embryogenesis. The results that the predicted aggregation domains in the localization signal of Buc are not required for germ plasm localization are in line with a more liquid-like behavior of germ plasm during embryogenesis, compared to oogenesis. The more solid Buc aggregates during oogenesis are probably required for the storage of maternally provided components, which is in line with our data and previous studies (Boke et al., 2016). In contrast, the function of the more liquid-like Buc aggregates can be to localize germ plasm and to release the maternally provided cellular structures into the embryo.

We demonstrated that germ plasm is not phosphorylated during embryogenesis. Moreover, BucLoc tyrosine phosphomimic does not localize to endogenous germ plasm and is expressed at lower levels than WT BucLoc. Hence, we assume that dephosphorylation of BucLoc at tyrosines might be required for germ plasm localization. Furthermore, we hypothesize that the tyrosine phosphorylation in BucLoc might be required for Buc degradation. Identifying the exact tyrosine residues which are differentially phosphorylated in BucLoc between oogenesis and embryogenesis will be the crucial step in understanding the role of phosphorylation in Buc localization or degradation.

Using the BucLoc domain we identified 23 proteins in the MS analysis. From the identified BucLoc interactors, Ihabp4 is the most promising candidate for anchoring germ plasm to cytoskeleton. Furthermore, G3bp2, Rbm4.3. and Buc2l are less likely to play a role in germ plasm localization, because they have no known domains or data on interaction with cytoskeleton. Future study will focus on co-localization analysis of Buc and Ihabp4.

In this study, we established an assay to study co-localization of overexpressed germ plasm organizers like Velo1, Oskar and Buc with the endogenous zebrafish germ plasm. Our results show the conservation of the germ plasm localization mechanism in vertebrates. Hence, we hypothesize that in other vertebrates like Xenopus, tight junction also plays a role in germ plasm localization. Studying the co-localization of Xenopus germ plasm and ZO1 will be an essential step in further understanding of the evolutionary conservation of the germ plasm localization mechanism in vertebrates.

Identification of ZO1-A as a potential candidate for anchoring germ plasm, addressing the role of PrDs and IDRs in germ plasm localization, isolation of the core Buc interactome and studying the conservation of the germ plasm localization mechanism among vertebrates and invertebrates contribute to understanding of the fundamental principles of germ plasm localization. 


\section{Methods}

\subsection{Zebrafish Handling \& Maintenance}

Zebrafish (Danio rerio) was used as a model organism and maintained due to the standard protocol (Westerfield, 2000).

\subsubsection{Microinjection}

Before injection, microneedles were pulled using a P-97 Micropipette Puller (Sutter Instrument Flaming, Novato, USA) with the following settings: heat $\left(515^{\circ} \mathrm{C}\right)$, pull $(218)$, velocity $(80)$ and time (250 ms). A purified sense RNA was diluted as described in Table 2 (final RNA concentration: 120 $\mathrm{ng} / \mu \mathrm{l})$. Then, microneedles were mounted in the needle holder connected to a PV820 Pneumatic Pico Pump (World Precision Instruments, Sarasota, USA) and loaded with the capped sense RNA. The tip of the microneedle was clipped with a forceps and injection volume was adjusted to $4 \mathrm{nl}$ by manipulating the injection pressure and time. After injection, embryos were transferred back to E3 (5 $\mathrm{mM} \mathrm{NaCl}, 0.17 \mathrm{mM} \mathrm{KCl}, 0.33 \mathrm{mM} \mathrm{CaCl}, 0.33 \mathrm{mM} \mathrm{MgSO} 4,0.00001 \%$ methylene blue) medium and incubated at RT or at $28^{\circ} \mathrm{C}$.

Table 2: RNA dilution for microinjection

\begin{tabular}{ll}
\hline Volume $(\mu \mathrm{l})$ & \\
\hline $\mathrm{x} \mu \mathrm{l}$ & purified RNA \\
$1 \mu \mathrm{l}$ & $1 \mathrm{M} \mathrm{KCl}$ \\
$1 \mu \mathrm{l}$ & $0.5 \%$ Phenolred \\
\hline
\end{tabular}

Up to $10 \mathrm{ul} \quad$ RNAse-free $\mathrm{H} 2 \mathrm{O}$

\subsubsection{Dechorionation}

Embryos were chemically dechorionated with pronase enzyme $(30 \mathrm{mg} / \mathrm{ml}$; Roche, Mannheim). Embryos were treated for 3-5 min with pronase solution in glass beakers $(\approx 50 \mu 1 \mathrm{E} 3$ medium $+50 \mu \mathrm{l}$ of the pronase solution) and afterwards washed three times with 1x E3 medium.

\subsubsection{Deyolking}

Up to 200 embryos were deyolked to remove most of the yolk granules before lysis (Link et al., 2006). For deyolking $1 / 2$ Ginzburg Fish Ringer with Calcium was used $(55 \mathrm{mM} \mathrm{NaCl}, 2.7 \mathrm{mM} \mathrm{CaCl}$, $1.8 \mathrm{mM} \mathrm{KCl}, 1.25 \mathrm{mM} \mathrm{NaHCO} 3)$. After deyolking the cell pellet was washed two times with wash buffer (110 mM NaCl, $3.5 \mathrm{mM} \mathrm{KCl}, 2.7 \mathrm{mM} \mathrm{CaCl} 2,10 \mathrm{mM}$ Tris (pH 8.5)). The cell pellet was directly used for further experiments. 


\subsubsection{Preparation of embryo lysates}

To prepare embryo lysates for analysis by SDS-polyacrylamide gel electrophoresis (Chapter 5.4.1), deyolked embryo were resuspended in 2x SDS loading buffer (4\% SDS, $20 \%$ glycerol, $10 \%$ 2mercaptoethanol, $0.004 \%$ bromophenol blue and $0.125 \mathrm{M}$ Tris $\mathrm{HCl}, \mathrm{pH}$ approx. 6.8., Merck, Kenilworth, New Jersey, USA) and incubated at $95{ }^{\circ} \mathrm{C}$ for $5 \mathrm{~min}$. After cooling of the sample on ice, it was loaded directly on a SDS-polyacrylamide gel or stored at $-20^{\circ} \mathrm{C}$.

\subsubsection{Live imaging}

Embryos were incubated until 512-cell stage (2.5 hpf), dechorionated (Chapter 5.1.3), transferred to a $35 \mathrm{~mm}$ FluoroDish (World Precision Instruments Inc., Sarasota, USA) and analyzed with a LSM780 confocal microscope using the Zen acquisition software (Zeiss AG, Jena, Germany).

\subsubsection{Drug treatments}

The treatment of oocytes and embryos with hexanediol and hexanetriol was performed as described in Krishnakumar et al., 2018. For the Calphostin C treatment, embryos were placed at the 1-cell stage in either $500 \mathrm{nM}$ or $3 \mu \mathrm{M}$ drug dissolved in the E3 medium. Then embryos were processed for the live-imaging (Chapter 5.1.6) at the 512-cell stage.

\subsection{Plasmid vectors and constructs}

\subsubsection{Plasmid vectors}

PCs2+ vector contains the simian cytomegalovirus IE94 enhancer/promoter that functions in vivo (e.g. frog and zebrafish) as well as tissue culture cells (Rupp et al., 1994). In vitro transcription of sense RNA is possible using the SP6 promoter in 5'-untranslated region of the IE94 driven transcript in pCS2+ (Rupp et al., 1994). The plasmid carries ampicillin resistance and the vector backbone originates from the pBluescript II KS+ vector. pDONR221 (Life Technologies, Carlsbad, USA) contains recombination sites for bacteriophage lambda in E.coli. These recombination sites are used in the Gateway ${ }^{\circledR}$ cloning (Chapter 5.3.5). pDONR221 contains a kanamycin resistance gene for selection in E.coli. pCSDest 2 is a Gateway-adapted expression vector, based on the pCS-backbone (Villefranc et al., 2007). To generate C-terminal GFP or mCherry tags, pCSDest 2 can be combined with p3EeGFP and p3EmCherry gateway-adapted entry vectors in a multisite gateway recombination reaction (Villefranc et al., 2007).

\subsubsection{Cloned vector and expression constructs}

Expression constructs to study Buc and sOsk aggregation in vitro were cloned with the In-Fusion ${ }^{\circledR}$ cloning (Chapter 5.3.6). The constructs used for the identification, mapping and mutagenesis of the BucLoc domain were cloned by the Gateway® cloning (Chapter 5.3.5). 
Table 3: Cloned expression plasmids used for in vitro transcription of Buc deletion constructs or transfection into cell culture cells.

\begin{tabular}{|c|c|c|c|}
\hline Name & Vector & Insert & Cloning strategy \\
\hline $\begin{array}{l}\text { pCS2+ buc- } \\
\text { pmEGFP }\end{array}$ & pCS2+ & pmEGFP & $\begin{array}{l}\text { (1) Buc was amplified using the primers } \\
\text { pCS2+_Buc_fl_fw_BamhI, } \\
\text { pCS2+_Buc_fl_rev_BamhI from pCSdest2-Buc- } \\
\text { GFP plasmid and inserted in the BamhI digested } \\
\text { pCS2+ plasmid by In-Fusion }{ }^{\circledR} \text { cloning. (2) pmEGFP } \\
\text { was amplified from pmEGFP-C1 (Addgene, plasmid } \\
\text { \#36412, Watertown, Massachusetts, USA) using the } \\
\text { primers pmGFP_in-fusion_fw, pmGFP_in- } \\
\text { fusion_rev and inseted in the pCS2+ Buc digested } \\
\text { with XbaI by In-Fusion } ® \text { cloning. }\end{array}$ \\
\hline $\begin{array}{l}\text { pCS2+ } \\
\text { sOsk- } \\
\text { pmEGFP }\end{array}$ & pCS2+ & pmEGFP & $\begin{array}{l}\text { (1) sOsk was amplified using primers } \\
\text { Osk_fw_in_fusion_pCS2+ } \\
\text { Osk_rev_in_fusion_pCS2+ BamHI primers and } \\
\text { inserted in the BamhI digested pCS2+ plasmid by In- } \\
\text { Fusion }{ }^{\circledR} \text { cloning. (2) pmEGFP was amplified using } \\
\text { the primers pmGFP_in-fusion_fw, pmGFP_in- } \\
\text { fusion_rev and inserted in the pCS2+ sOsk digested } \\
\text { with XbaI by In-Fusion }{ }^{\circledR} \text { cloning. }\end{array}$ \\
\hline $\begin{array}{l}\text { pENTR221- } \\
\text { sOsk }\end{array}$ & $\begin{array}{l}\mathrm{pDONR} \\
221\end{array}$ & sOsk & $\begin{array}{l}\text { sOsk sequence was amplified from pCS2+ sOsk- } \\
\text { eGFP, using the primers Osk_gateway_fw, } \\
\text { Osk_gateway_rev. The PCR product was recombined } \\
\text { into pDONR221. }\end{array}$ \\
\hline $\begin{array}{l}\text { pEXPpCSD } \\
\text { est } 2 \\
\text { sOsk- } \\
\text { mCherry }\end{array}$ & $\begin{array}{l}\text { pCSdest } \\
2\end{array}$ & $\begin{array}{l}\text { sOsk- } \\
\text { mCherry }\end{array}$ & $\begin{array}{l}\text { pENTR221-sOsk was recombined with pCSDest2 } \\
\text { and p3EmCherry }\end{array}$ \\
\hline $\begin{array}{l}\text { pENTR221- } \\
\text { Buc21-88 }\end{array}$ & $\begin{array}{l}\text { pDONR } \\
221\end{array}$ & buc21-88 & $\begin{array}{l}\text { buc21-88 was amplified using the primers Buc11- } \\
\text { 88_-10_N_fw_gateway, } \\
\text { 88_WT_rev_gateway from the pCSdest2-Buc11-88- } \\
\text { eGFP. The PCR product was recombined into } \\
\text { pDONR221. }\end{array}$ \\
\hline $\begin{array}{l}\text { pENTR221- } \\
\text { Buc31-88 }\end{array}$ & $\begin{array}{l}\text { pDONR } \\
221\end{array}$ & buc31-88 & $\begin{array}{l}\text { buc31-88 was amplified using the primers Buc11- } \\
\text { 88_-20_N_fw_gateway, } \\
\text { 88_WT_rev_gateway from the pCSdest2-Buc11-88- } \\
\text { eGFP. The PCR product was recombined into } \\
\text { pDONR221. }\end{array}$ \\
\hline $\begin{array}{l}\text { pENTR221- } \\
\text { Buc36-88 }\end{array}$ & $\begin{array}{l}\text { pDONR } \\
221\end{array}$ & buc36-88 & $\begin{array}{l}\text { buc36-88 was amplified using the primers Buc11- } \\
\text { 88_-25_N_fw_gateway, } \\
\text { 88_WT_rev_gateway from the pCSdest2-Buc11-88- } \\
\text { eGFP. The PCR product was recombined into } \\
\text { pDONR221. }\end{array}$ \\
\hline
\end{tabular}




\begin{tabular}{|c|c|c|c|}
\hline $\begin{array}{l}\text { pENTR221- } \\
\text { Buc31-78 }\end{array}$ & $\begin{array}{l}\text { pDONR } \\
221\end{array}$ & buc31-78 & $\begin{array}{l}\text { Buc11-78 was amplified using the primers Buc11- } \\
\text { 88_-10_C_fw_gateway, } \\
\text { 20_N_fw_gateway from the pCSdest2-Buc11-88- } \\
\text { eGFP. The PCR product was recombined into } \\
\text { pDONR221. }\end{array}$ \\
\hline $\begin{array}{l}\text { pENTR221- } \\
\text { Buc31-73 }\end{array}$ & $\begin{array}{l}\text { pDONR } \\
221\end{array}$ & buc31-73 & $\begin{array}{l}\text { buc11-73 was amplified using the primers Buc11- } \\
\text { 88_-15_C_fw_gateway, } \\
\text { 20_N_fw_gateway from the pCSdest2-Buc11-88- } \\
\text { eGFP. The PCR product was recombined into } \\
\text { pDONR221. }\end{array}$ \\
\hline $\begin{array}{l}\text { pEXPpCSD } \\
\text { est2 } \\
\text { buc21-88- } \\
\text { eGFP }\end{array}$ & $\begin{array}{l}\text { pCSdest } \\
2\end{array}$ & $\begin{array}{l}\text { buc21-88- } \\
\text { eGFP }\end{array}$ & $\begin{array}{l}\text { pENTR221 buc21-88 was recombined with } \\
\text { pCSDest } 2 \text { and } \mathrm{p} 3 \mathrm{EeGFP}\end{array}$ \\
\hline $\begin{array}{l}\text { pEXPpCSD } \\
\text { est } 2 \\
\text { buc31-88- } \\
\text { eGFP }\end{array}$ & $\begin{array}{l}\text { pCSdest } \\
2\end{array}$ & $\begin{array}{l}\text { Buc31-88- } \\
e G F P\end{array}$ & $\begin{array}{lclll}\text { pENTR221 buc31-88 } & \text { was } & \text { recombined with } \\
\text { pCSDest } 2 \text { and } \mathrm{p} 3 \mathrm{EeGFP} & & & \end{array}$ \\
\hline $\begin{array}{l}\text { pEXPpCSD } \\
\text { est2 } \\
\text { buc36-88- } \\
\text { eGFP }\end{array}$ & $\begin{array}{l}\text { pCSdest } \\
2\end{array}$ & $\begin{array}{l}\text { buc36-88- } \\
\text { eGFP }\end{array}$ & $\begin{array}{lcccc}\text { pENTR221 buc36-88 } & \text { was } & \text { recombined with } \\
\text { pCSDest } 2 \text { and p3EeGFP } & & & \end{array}$ \\
\hline $\begin{array}{l}\text { pEXPpCSD } \\
\text { est2 } \\
\text { buc31-78- } \\
\text { eGFP }\end{array}$ & $\begin{array}{l}\text { pCSdest } \\
2\end{array}$ & $\begin{array}{l}\text { buc31-78- } \\
e G F P\end{array}$ & $\begin{array}{lclll}\text { pENTR221 buc11-78 } & \text { was } & \text { recombined with } \\
\text { pCSDest2 and p3EeGFP } & & & \end{array}$ \\
\hline $\begin{array}{l}\text { pEXPpCSD } \\
\text { est2 } \\
\text { buc31-73- } \\
\text { eGFP }\end{array}$ & $\begin{array}{l}\text { pCSdest } \\
2\end{array}$ & $\begin{array}{l}\text { buc31-73- } \\
\text { eGFP }\end{array}$ & $\begin{array}{lclll}\text { pENTR221 buc11-73 was } & \text { recombined with } \\
\text { pCSDest } 2 \text { and } \mathrm{p} 3 \mathrm{EeGFP} & & & \end{array}$ \\
\hline $\begin{array}{l}\text { pEXPpCSD } \\
\text { est } 2 \\
\text { buc31-78- } \\
\text { mCherry }\end{array}$ & $\begin{array}{l}\text { pCSdest } \\
2\end{array}$ & $\begin{array}{l}\text { buc31-78- } \\
\text { mCherry }\end{array}$ & $\begin{array}{l}\text { pENTR221-buc31-78-mCherry was recombined with } \\
\text { pCSDest } 2 \text { and p3EmCherry }\end{array}$ \\
\hline
\end{tabular}




\begin{tabular}{|c|c|c|c|}
\hline $\begin{array}{l}\text { pEXPpCSD } \\
\text { est2 } \\
\text { buc31-73- } \\
\text { mCherry }\end{array}$ & $\begin{array}{l}\text { pCSdest } \\
2\end{array}$ & $\begin{array}{l}\text { buc31-73- } \\
\text { mCherry }\end{array}$ & $\begin{array}{l}\text { pENTR221-buc31-73-mCherry was recombined with } \\
\text { pCSDest2 and p3EmCherry }\end{array}$ \\
\hline $\begin{array}{l}\text { pENTR221 } \\
\text { Buc31-78- } \\
\Delta 31-35\end{array}$ & $\begin{array}{l}\text { pDONR } \\
221\end{array}$ & $\begin{array}{l}\text { buc31-78- } \\
431-35\end{array}$ & $\begin{array}{l}\text { buc31-78-431-35 was amplified using the primers } \\
\text { Buc31-78_.431-35_fw_gateway, } \\
\text { 78_rev_gateway from the extended overlapping } \\
\text { oligos: Buc31-78_ } \Delta 31-35 \_f w \text { and Buc_57-78_rev. } \\
\text { The PCR product was recombined into pDONR221 }\end{array}$ \\
\hline $\begin{array}{l}\text { pENTR221 } \\
\text { Buc31-78- } \\
\Delta 36-41\end{array}$ & $\begin{array}{l}\text { pDONR } \\
221\end{array}$ & $\begin{array}{l}\text { buc31-78- } \\
\Delta 36-41\end{array}$ & $\begin{array}{l}\text { buc31-78-436-41 was amplified using the primers } \\
\text { Buc31-78_fw_gateway, Buc31-78_rev_gateway } \\
\text { from the extended overlapping oligos: Buc31- } \\
78 \_\Delta 36-41 \_ \text {fw and Buc_57-78_rev. The PCR } \\
\text { product was recombined into pDONR221 }\end{array}$ \\
\hline $\begin{array}{l}\text { pENTR221 } \\
\text { Buc31-78- } \\
\Delta 42-46\end{array}$ & $\begin{array}{l}\text { pDONR } \\
221\end{array}$ & $\begin{array}{l}\text { buc } 31-78- \\
\Delta 42-46\end{array}$ & $\begin{array}{l}\text { buc31-78-442-46 was amplified using the primers } \\
\text { Buc31-78_fw_gateway, Buc31-78_rev_gateway } \\
\text { from the extended overlapping oligos: Buc31- } \\
78 \_\Delta 42-46 \_f w \text { and Buc_57-78_rev. The PCR } \\
\text { product was recombined into pDONR221 }\end{array}$ \\
\hline $\begin{array}{l}\text { pENTR221 } \\
\text { Buc31-78- } \\
\Delta 47-51\end{array}$ & $\begin{array}{l}\text { pDONR } \\
221\end{array}$ & $\begin{array}{l}\text { buc31-78- } \\
447-51\end{array}$ & $\begin{array}{l}\text { buc31-78-447-51 was amplified using the primers } \\
\text { Buc31-78_fw_gateway, Buc31-78_rev_gateway } \\
\text { from the extended overlapping oligos: Buc31- } \\
78 \_\Delta 47-51 \_ \text {fw and Buc_57-78_rev. The PCR } \\
\text { product was recombined into pDONR221 }\end{array}$ \\
\hline $\begin{array}{l}\text { pENTR221 } \\
\text { Buc31-78- } \\
\Delta 52-56\end{array}$ & $\begin{array}{l}\text { pDONR } \\
221\end{array}$ & $\begin{array}{l}\text { buc31-78- } \\
\Delta 52-56\end{array}$ & $\begin{array}{l}\text { buc31-78-452-56 was amplified using the primers } \\
\text { Buc31-78_fw_gateway, Buc31-78_rev_gateway } \\
\text { from the extended overlapping oligos: Buc31- } \\
78 \_\Delta 52-56 \_ \text {fw and Buc_57-78_rev. The PCR } \\
\text { product was recombined into pDONR221 }\end{array}$ \\
\hline $\begin{array}{l}\text { pENTR221 } \\
\text { Buc31-78- } \\
\Delta 57-61\end{array}$ & $\begin{array}{l}\text { pDONR } \\
221\end{array}$ & $\begin{array}{l}\text { buc31-78- } \\
457-61\end{array}$ & $\begin{array}{l}\text { buc31-78-457-61 was amplified using the primers } \\
\text { Buc31-78_fw_gateway, Buc31-78_rev_gateway } \\
\text { from the extended overlapping oligos: BucLoc_ } \Delta 57- \\
\text { 61_rev, Buc_31-51_fw. The PCR product was } \\
\text { recombined into pDONR221 }\end{array}$ \\
\hline $\begin{array}{l}\text { pENTR221 } \\
\text { Buc31- } \\
78 \_\Delta 62-66\end{array}$ & $\begin{array}{l}\text { pDONR } \\
221\end{array}$ & $\begin{array}{l}\text { buc31-78- } \\
\Delta 62-66\end{array}$ & $\begin{array}{l}\text { buc31-78-462-66 was amplified using the primers } \\
\text { Buc31-78_fw_gateway, Buc31-78_rev_gateway } \\
\text { from the extended overlapping oligos: BucLoc_ } \Delta 62- \\
\text { 66_rev, Buc_31-51_fw. The PCR product was } \\
\text { recombined into pDONR221 }\end{array}$ \\
\hline $\begin{array}{l}\text { pENTR221 } \\
\text { Buc31-78- } \\
\Delta 67-71\end{array}$ & $\begin{array}{l}\text { pDONR } \\
221\end{array}$ & $\begin{array}{l}\text { buc31-78- } \\
\Delta 67-71\end{array}$ & $\begin{array}{l}\text { buc31-78-467-71 was amplified using the primers } \\
\text { Buc31-78_fw_gateway, Buc31-78_rev_gateway } \\
\text { from the extended overlapping oligos: BucLoc_ } \Delta 67- \\
\text { 71_rev, Buc_31-51_fw. The PCR product was } \\
\text { recombined into pDONR221 }\end{array}$ \\
\hline
\end{tabular}




\begin{tabular}{|c|c|c|c|}
\hline $\begin{array}{l}\text { pEXPpCSD } \\
\text { est2 } \\
\text { Buc31-78- } \\
\Delta 31-35- \\
\text { mCherry }\end{array}$ & $\begin{array}{l}\text { pCSdest } \\
2\end{array}$ & $\begin{array}{l}\text { buc31-78- } \\
\text { 431-35- } \\
\text { Cherry }\end{array}$ & $\begin{array}{l}\text { pENTR221-buc31-78- } \Delta 31-35 \text { was recombined with } \\
\text { pCSDest } 2 \text { and p3EmCherry }\end{array}$ \\
\hline $\begin{array}{l}\text { pEXPpCSD } \\
\text { est2 } \\
\text { Buc31-78- } \\
\Delta 36-41- \\
\text { mCherry }\end{array}$ & $\begin{array}{l}\text { pCSdest } \\
2\end{array}$ & $\begin{array}{l}\text { buc31-78- } \\
\Delta 36-41- \\
\text { Cherry }\end{array}$ & $\begin{array}{l}\text { pENTR221-buc31-78- } \Delta 36-41 \text { was recombined with } \\
\text { pCSDest } 2 \text { and p3EmCherry }\end{array}$ \\
\hline $\begin{array}{l}\text { pEXPpCSD } \\
\text { est2 } \\
\text { Buc31-78- } \\
\Delta 42-46- \\
\text { mCherry } \\
\end{array}$ & $\begin{array}{l}\text { pCSdest } \\
2\end{array}$ & $\begin{array}{l}\text { buc31-78- } \\
442-46- \\
\text { Cherry }\end{array}$ & $\begin{array}{l}\text { pENTR221-buc31-78- } \Delta 42-46 \text { was recombined with } \\
\text { pCSDest } 2 \text { and p3EmCherry }\end{array}$ \\
\hline $\begin{array}{l}\text { pEXPpCSD } \\
\text { est2 } \\
\text { Buc31-78- } \\
\Delta 47-51- \\
\text { mCherry }\end{array}$ & $\begin{array}{l}\text { pCSdest } \\
2\end{array}$ & $\begin{array}{l}\text { buc31-78- } \\
447-51- \\
\text { Cherry }\end{array}$ & $\begin{array}{l}\text { pENTR221-buc31-78- } \Delta 47-51 \text { was recombined with } \\
\text { pCSDest } 2 \text { and p3EmCherry }\end{array}$ \\
\hline $\begin{array}{l}\text { pEXPpCSD } \\
\text { est2 } \\
\text { Buc31-78- } \\
\Delta 52-56- \\
\text { mCherry } \\
\end{array}$ & $\begin{array}{l}\text { pCSdest } \\
2\end{array}$ & $\begin{array}{l}\text { buc31-78- } \\
\text { 452-56- } \\
\text { Cherry }\end{array}$ & $\begin{array}{l}\text { pENTR221-buc31-78- } \triangle 52-56 \text { was recombined with } \\
\text { pCSDest } 2 \text { and p3EmCherry }\end{array}$ \\
\hline $\begin{array}{l}\text { pEXPpCSD } \\
\text { est2 } \\
\text { Buc31-78- } \\
\Delta 57-61- \\
\text { mCherry }\end{array}$ & $\begin{array}{l}\text { pCSdest } \\
2\end{array}$ & $\begin{array}{l}\text { buc31-78- } \\
\text { 457-61- } \\
\text { Cherry }\end{array}$ & $\begin{array}{l}\text { pENTR221-buc31-78- } \triangle 57-61 \text { was recombined with } \\
\text { pCSDest } 2 \text { and p3EmCherry }\end{array}$ \\
\hline $\begin{array}{l}\text { pEXPpCSD } \\
\text { est2 } \\
\text { Buc31-78- } \\
\Delta 62-66- \\
\text { mCherry } \\
\end{array}$ & $\begin{array}{l}\text { pCSdest } \\
2\end{array}$ & $\begin{array}{l}\text { buc31-78- } \\
\text { 462-66- } \\
\text { Cherry }\end{array}$ & $\begin{array}{l}\text { pENTR221-buc31-78- } \Delta 62-66 \text { was recombined with } \\
\text { pCSDest } 2 \text { and p3EmCherry }\end{array}$ \\
\hline $\begin{array}{l}\text { pEXPpCSD } \\
\text { est2 } \\
\text { Buc31-78- } \\
\Delta 67-71- \\
\text { mCherry }\end{array}$ & $\begin{array}{l}\text { pCSdest } \\
2\end{array}$ & $\begin{array}{l}\text { buc31-78- } \\
\text { 467-71- } \\
\text { Cherry }\end{array}$ & $\begin{array}{l}\text { pENTR221-buc31-78- } \Delta 67-71 \\
\text { was recombined with pCSDest } 2 \text { and p3EmCherry }\end{array}$ \\
\hline pENTR221 & $\begin{array}{l}\text { pDONR } \\
221\end{array}$ & $\begin{array}{l}\text { buc31-78- } \\
\text { S33E }\end{array}$ & $\begin{array}{l}\text { buc31-78-S33E was amplified using the primers } \\
\text { Buc31-78_S33E_fw_gateway, } \\
\text { Buc31- }\end{array}$ \\
\hline
\end{tabular}




\begin{tabular}{|c|c|c|c|}
\hline $\begin{array}{l}\text { Buc31-78- } \\
\text { S33E }\end{array}$ & & & $\begin{array}{l}\text { 78_rev_gateway from the extended overlapping } \\
\text { oligos Buc_S33E_fw and Buc_57-78_rev.The PCR } \\
\text { product was recombined into pDONR221 }\end{array}$ \\
\hline $\begin{array}{l}\text { pENTR221 } \\
\text { Buc31-78- } \\
\text { S33A }\end{array}$ & $\begin{array}{l}\text { pDONR } \\
221\end{array}$ & $\begin{array}{l}\text { buc31-78- } \\
\text { S33A }\end{array}$ & $\begin{array}{l}\text { buc31-78-S33A was amplified using the primers } \\
\text { Buc31-78_S33A_fw_gateway, } \\
\text { 78_rev_gateway from the extended overlapping } \\
\text { oligos Buc_S33A_fw and Buc_57-78_rev. The PCR } \\
\text { product was recombined into pDONR221 }\end{array}$ \\
\hline $\begin{array}{l}\text { pENTR221 } \\
\text { Buc31-78- } \\
\text { allYtoE }\end{array}$ & $\begin{array}{l}\text { pDONR } \\
221\end{array}$ & $\begin{array}{l}\text { buc31-78- } \\
\text { allYtoE }\end{array}$ & $\begin{array}{l}\text { buc31-78-allYtoE was amplified using the primers } \\
\text { Buc31-78__allYtoE_fw_gateway, Buc31-78_ } \\
\text { allYtoE_rev_gateway from the extended overlapping } \\
\text { oligos Buc_allYtoE_fw and Buc_AllYtoE_rev. The } \\
\text { PCR product was recombined into pDONR221 }\end{array}$ \\
\hline $\begin{array}{l}\text { pENTR221 } \\
\text { Buc31-78- } \\
\text { allYtoF }\end{array}$ & $\begin{array}{l}\text { pDONR } \\
221\end{array}$ & $\begin{array}{l}\text { buc31-78- } \\
\text { allYtoF }\end{array}$ & $\begin{array}{l}\text { buc31-78-allYtoF was amplified using the primers } \\
\text { Buc31-78__allYtoF_fw_gateway, Buc31-78_ } \\
\text { allYtoF_rev_gateway from the extended overlapping } \\
\text { oligos: Buc_AllYtoF_fw and Buc_AllYtoF_rev. The } \\
\text { PCR product was recombined into pDONR221 }\end{array}$ \\
\hline $\begin{array}{l}\text { pEXPpCSD } \\
\text { est2 } \\
\text { Buc31-78- } \\
\text { S33E- } \\
\text { mCherry }\end{array}$ & $\begin{array}{l}\text { pCSdest } \\
2\end{array}$ & $\begin{array}{l}\text { buc31-78- } \\
\text { S33E- } \\
\text { mCherry }\end{array}$ & $\begin{array}{l}\text { pENTR221-buc31-78-S33E } \\
\text { was recombined with pCSDest2 and p3EmCherry }\end{array}$ \\
\hline $\begin{array}{l}\text { pEXPpCSD } \\
\text { est2 } \\
\text { Buc31-78- } \\
\text { S33A- } \\
\text { mCherry }\end{array}$ & $\begin{array}{l}\text { pCSdest } \\
2\end{array}$ & $\begin{array}{l}\text { buc31-78- } \\
\text { S33A- } \\
\text { mCherry }\end{array}$ & $\begin{array}{l}\text { pENTR221-buc31-78-S33A } \\
\text { was recombined with pCSDest2 and p3EmCherry }\end{array}$ \\
\hline $\begin{array}{l}\text { pEXPpCSD } \\
\text { est2 } \\
\text { Buc31-78- } \\
\text { AllYtoE- } \\
\text { mCherry }\end{array}$ & $\begin{array}{l}\text { pCSdest } \\
2\end{array}$ & $\begin{array}{l}\text { buc31-78- } \\
\text { AllYtoE- } \\
\text { mCherry }\end{array}$ & $\begin{array}{l}\text { pENTR221-buc31-78-AllYtoE } \\
\text { was recombined with pCSDest2 and p3EmCherry }\end{array}$ \\
\hline $\begin{array}{l}\text { pEXPpCSD } \\
\text { est2 } \\
\text { Buc31-78- } \\
\text { AllYtoF- } \\
\text { mCherry }\end{array}$ & $\begin{array}{l}\text { pCSdest } \\
2\end{array}$ & $\begin{array}{l}\text { buc31-78- } \\
\text { AllYtoF- } \\
\text { mCherry }\end{array}$ & $\begin{array}{l}\text { pENTR221-buc31-78-AllYtoF } \\
\text { was recombined with pCSDest2 and p3EmCherry }\end{array}$ \\
\hline $\begin{array}{l}\text { pENTR221 } \\
\text { Buc31-78- } \\
66,68,71 Y \text { F } \\
Y>\text { DDD }\end{array}$ & $\begin{array}{l}\text { pDONR } \\
221\end{array}$ & $\begin{array}{l}\text { buc31-78- } \\
66,68,71 Y F Y \\
>D D D\end{array}$ & $\begin{array}{l}\text { buc31-78-66,68,71YFY >DDD was amplified by a } \\
\text { standard high-fidelity PCR from pDONR221-buc31- } \\
78 \text { using the primers Buc_66_68_71_YFY } \\
\text { DDD_fw_5'phosphorylated } \\
\text { Buc_66_68_71_YFY } \\
\text { DDD_rev_5'phosphorylated. Then the amplified } \\
\text { DNA was ligated (Chapter 5.3.4) and correct } \\
\text { plasmids were selected by colony PCR. }\end{array}$ \\
\hline
\end{tabular}


Table 4: Primers used for the in-fusion and gateway cloning

\begin{tabular}{|c|c|c|}
\hline $\begin{array}{l}\text { Nam } \\
\text { e }\end{array}$ & Sequence (5' to 3' direction) & $\begin{array}{l}\text { Cloning } \\
\text { purpose }\end{array}$ \\
\hline pCS2+_Buc_fl_fw_BamhI & $\begin{array}{l}\text { TCTTTTTGCAGGATCATGGAAGG } \\
\text { AATAAATAACAATTCACAACCAA } \\
\text { TGGGAGT }\end{array}$ & $\begin{array}{l}\text { Buc into } \\
\text { pCS2+ }\end{array}$ \\
\hline pCS2+_Buc_fl_rev_BamhI & $\begin{array}{l}\text { CGAATCGATGGGATCGTATCTTG } \\
\text { AGCCTCTTTTCTTCATAGAACCTC }\end{array}$ & $\begin{array}{l}\text { Buc into } \\
\text { pCS2+ }\end{array}$ \\
\hline Osk_fw_in_fusion_pCS2+ BamHI & $\begin{array}{l}\text { TTGTTCTTTTTGCAGGATC } \\
\text { ATGACCATCATCGAGAGCAAC }\end{array}$ & $\begin{array}{l}\text { sOsk into } \\
\text { pCS2+ }\end{array}$ \\
\hline Osk_rev_in_fusion_pCS2+BamHI & $\begin{array}{l}\text { TCGAATCGATGGGATC } \\
\text { GTGGTATGTTCTCCAGGGACGG }\end{array}$ & $\begin{array}{l}\text { sOsk into } \\
\text { pCS2+ }\end{array}$ \\
\hline mGFP_in-fusion_fw & $\begin{array}{l}\text { AGGCCTCTCGAGCCTCTAGATGG } \\
\text { TGAGCAAGGGCGAGGA }\end{array}$ & $\begin{array}{l}\text { C-term. } \\
\text { Fusion f } \\
\text { pmEGFP } \\
\text { with Buc / } \\
\text { sOsk }\end{array}$ \\
\hline mGFP_in-fusion_rev & $\begin{array}{l}\text { CGACTCACTATAGTTCTAGTTACT } \\
\text { TGTACAGCTCGTCCAT }\end{array}$ & $\begin{array}{l}\text { C-term. } \\
\text { Fusion } \\
\text { pmEGFP } \\
\text { with Buc / } \\
\text { sOsk }\end{array}$ \\
\hline Osk_gateway_fw & $\begin{array}{l}\text { GGGGACAAGTTTGTACAAAAAAG } \\
\text { CAGGCTATATGACCATCATCGAG } \\
\text { AGCAAC }\end{array}$ & $\begin{array}{l}\text { sOsk } \\
\text { mCherry C- } \\
\text { term. fusion }\end{array}$ \\
\hline Osk_gateway_rev & $\begin{array}{l}\text { GGGGACCACTTTGTACAAGAAAG } \\
\text { CTGGGTA } \\
\text { GTGGTATGTTCTCCAGGGACGG }\end{array}$ & $\begin{array}{l}\text { sOsk } \\
\text { mCherry C- } \\
\text { term. fusion }\end{array}$ \\
\hline Buc11-88_-10_N_fw_gateway & $\begin{array}{l}\text { GGGGACAAGTTTGTACAAAAAAG } \\
\text { CAGGCTATATGAACCACACAAGA } \\
\text { CCATTTTTC }\end{array}$ & $\begin{array}{l}\text { Buc } \\
\text { deletion }\end{array}$ \\
\hline Buc11-88_-20_N_fw_gateway & $\begin{array}{l}\text { GGGGACAAGTTTGTACAAAAAAG } \\
\text { CAGGCTAT } \\
\text { ATGCCACCATCTCAGCCTTATTT }\end{array}$ & $\begin{array}{l}\text { Buc } \\
\text { deletion }\end{array}$ \\
\hline
\end{tabular}




\begin{tabular}{|c|c|c|}
\hline Buc11-88_-25_N_fw_gateway & $\begin{array}{l}\text { GGGGACAAGTTTGTACAAAAAAG } \\
\text { CAGGCTATATGTATTTCATGTATC } \\
\text { AGTGGCC }\end{array}$ & $\begin{array}{l}\text { Buc } \\
\text { deletion }\end{array}$ \\
\hline Buc11-88_-10_C_rev_gateway & $\begin{array}{l}\text { GGGGACCACTTTGTACAAGAAAG } \\
\text { CTGGGTA } \\
\text { ATGTGGAATCACATAGCCAGG }\end{array}$ & $\begin{array}{l}\text { Buc } \\
\text { deletion }\end{array}$ \\
\hline Buc11-88_-15_C_rev_gateway & $\begin{array}{l}\text { GGGGACCACTTTGTACAAGAAAG } \\
\text { CTGGGTA } \\
\text { GCCAGGATACTGCATAAACTGAT } \\
\text { AAGGGGC }\end{array}$ & $\begin{array}{l}\text { Buc } \\
\text { deletion }\end{array}$ \\
\hline Buc11-88_WT_fw_gateway & $\begin{array}{lr}\text { GGGGACAAGTTTGTACAAAAAAG } \\
\text { CAGGCTAT } \\
\text { GGAGTTGGGCAACCTCA }\end{array}$ & $\begin{array}{l}\text { Buc } \\
\text { deletion }\end{array}$ \\
\hline Buc11-88_WT_rev_gateway & $\begin{array}{l}\text { GGGGACCACTTTGTACAAGAAAG } \\
\text { CTGGGTA } \\
\text { TCTTCTGTAATCAATTGGCTG }\end{array}$ & $\begin{array}{l}\text { Buc } \\
\text { deletion }\end{array}$ \\
\hline Buc31-78__31-35_fw_gateway & $\begin{array}{l}\text { GGGGACAAGTTTGTACAAAAAAG } \\
\text { CAGGCTATATGTATTTCATGTATC } \\
\text { AGTGG }\end{array}$ & $\begin{array}{l}\text { BucLoc } \\
\text { mapping }\end{array}$ \\
\hline Buc31-78_fw_gateway & $\begin{array}{l}\text { GGGGACAAGTTTGTACAAAAAAG } \\
\text { CAGGCTATATGCCACCATCTCAG } \\
\text { CCT }\end{array}$ & $\begin{array}{l}\text { BucLoc } \\
\text { mapping }\end{array}$ \\
\hline buc31-78_rev_gateway & $\begin{array}{l}\text { GGGGACCACTTTGTACAAGAAAG } \\
\text { CTGGGTA } \\
\text { ATGTGGAATCACATAGCC }\end{array}$ & $\begin{array}{l}\text { BucLoc } \\
\text { mapping }\end{array}$ \\
\hline Buc31-78_S33E_fw_gateway & $\begin{array}{l}\text { GGGGACAAGTTTGTACAAAAAAG } \\
\text { CAGGCTATATGCCACCAGAGCAG } \\
\text { CCTTAT }\end{array}$ & $\begin{array}{l}\text { BucLoc } \\
\text { mutagenesis }\end{array}$ \\
\hline Buc31-78_S33A_fw_gateway & $\begin{array}{l}\text { GGGACAAGTTTGTACAAAAAAGC } \\
\text { AGGCTATATGCCACCAGCTCAGC } \\
\text { CTTATTTC }\end{array}$ & $\begin{array}{l}\text { BucLoc } \\
\text { mutagenesis }\end{array}$ \\
\hline Buc31-78_allYtoE_fw_gateway & $\begin{array}{l}\text { GGGGACAAGTTTGTACAAAAAAG } \\
\text { CAGGCTATATGCCACCATCTCAG } \\
\text { CCTGAA }\end{array}$ & $\begin{array}{l}\text { BucLoc } \\
\text { mutagenesis }\end{array}$ \\
\hline Buc31-78_allYtoE_rev_gateway & $\begin{array}{l}\text { GGGGACCACTTTGTACAAGAAAG } \\
\text { CTGGGTAATGTGGAATCACTTCG } \\
\text { CCAGG }\end{array}$ & $\begin{array}{l}\text { BucLoc } \\
\text { mutagenesis }\end{array}$ \\
\hline Buc31-78_allYtoF_fw_gateway & $\begin{array}{l}\text { GGGGACAAGTTTGTACAAAAAAG } \\
\text { CAGGCTATATGCCACCATCTCAG } \\
\text { ССTTTT }\end{array}$ & $\begin{array}{l}\text { BucLoc } \\
\text { mutagenesis }\end{array}$ \\
\hline Buc31-78_allYtoF_rev_gateway & $\begin{array}{l}\text { GGGGACCACTTTGTACAAGAAAG } \\
\text { CTGGGTAATGTGGAATCACAAAG } \\
\text { CCAGG }\end{array}$ & $\begin{array}{l}\text { BucLoc } \\
\text { mutagenesis }\end{array}$ \\
\hline
\end{tabular}




\begin{tabular}{|l|l|l|}
\hline $\begin{array}{l}\text { Buc_66_68_71_YFY } \\
\text { DDD_fw_5'phosphorylated }\end{array}$ & $\begin{array}{l}\text { CCCTATATGGCCCCTGATCAGGAT } \\
\text { ATGCAG } \\
\text { GATCCTGGCTATGTGATT }\end{array}$ & $\begin{array}{l}\text { BucLoc } \\
\text { mutagenesis }\end{array}$ \\
\hline $\begin{array}{lll}\text { Buc_66_68_71_YFY } \\
\text { DDD_rev_5'phosphorylated }\end{array}$ & AATCACATAGCCAGGATCCTGCA & BucLoc \\
& TATCCTGATCAGGGGCCATATAG & mutagenesis \\
\end{tabular}

Table 5: Oligonucleotides used for BucLoc mapping and mutagenesis

\begin{tabular}{|c|c|c|}
\hline Name & Sequence (5' to $3^{\prime}$ direction) & Cloning purpose \\
\hline $\begin{array}{l}\text { BucLoc_s31- } \\
\text { 35_fw }\end{array}$ & $\begin{array}{l}\text { ATGTATTTCATGTATCAGTGGCCCATGAATCCA } \\
\text { TATGGCCATTACGGTTTTCCCGGGCCGGCTTTG } \\
\text { CACTTTGGCCGTCCCTAT }\end{array}$ & $\begin{array}{l}\text { BucLoc } \\
\text { mapping }\end{array}$ \\
\hline $\begin{array}{l}\text { BucLoc_s36- } \\
41 \_f w\end{array}$ & $\begin{array}{l}\text { ATGCCACCATCTCAGCCTCCCATGAATCCATAT } \\
\text { GGCCAT TAC GGT TTT CCC GGG CCG GCT TTG } \\
\text { CAC TTT GGC CGT CCC TAT ATG }\end{array}$ & $\begin{array}{l}\text { BucLoc } \\
\text { mapping }\end{array}$ \\
\hline $\begin{array}{l}\text { BucLoc_s42- } \\
46 \_f w\end{array}$ & $\begin{array}{l}\text { ATG CCA CCA TCT CAG CCT TAT TTC ATG TAT } \\
\text { CAG TGG GGC CAT TAC GGT TTT CCC GGG CCG } \\
\text { GCT TTG CAC TTT GGC CGT CCC TAT ATG }\end{array}$ & $\begin{array}{l}\text { BucLoc } \\
\text { mapping }\end{array}$ \\
\hline $\begin{array}{l}\text { BucLoc_ } \Delta 47- \\
51 \_f w\end{array}$ & $\begin{array}{l}\text { ATG CCA CCA TCT CAG CCT TAT TTC ATG TAT } \\
\text { CAG TGG CCC ATG AAT CCA TAT CCC GGG CCG } \\
\text { GCT TTG CAC TTT GGC CGT CCC TAT ATG }\end{array}$ & $\begin{array}{l}\text { BucLoc } \\
\text { mapping }\end{array}$ \\
\hline $\begin{array}{l}\text { BucLoc_ } \Delta 52- \\
56 \_f w\end{array}$ & $\begin{array}{l}\text { ATG CCA CCA TCT CAG CCT TAT TTC ATG TAT } \\
\text { CAG TGG CCC ATG AAT CCA TAT GGC CAT TAC } \\
\text { GGT TTT CAC TTT GGC CGT CCC TAT ATG }\end{array}$ & $\begin{array}{l}\text { BucLoc } \\
\text { mapping }\end{array}$ \\
\hline $\begin{array}{l}\text { BucLoc_ } \Delta 57- \\
\text { 61_rev }\end{array}$ & $\begin{array}{l}\text { ATGTGGAATCACATAGCCAGGATACTGCATAAA } \\
\text { CTGATAAGGGGCCATATACAAAGCCGGCCCGG } \\
\text { GAAAACCGTAATGGCCATATGG }\end{array}$ & $\begin{array}{l}\text { BucLoc } \\
\text { mapping }\end{array}$ \\
\hline $\begin{array}{l}\text { BucLoc_s62- } \\
\text { 66_rev }\end{array}$ & $\begin{array}{l}\text { ATGTGGAATCACATAGCCAGGATACTGCATAAA } \\
\text { CTGGGGACGGCCAAAGTGCAAAGCCGGCCCGG } \\
\text { GAAAACCGTAATGGCCATATGG }\end{array}$ & $\begin{array}{l}\text { BucLoc } \\
\text { mapping }\end{array}$ \\
\hline $\begin{array}{l}\text { BucLoc_s67- } \\
\text { 71_rev }\end{array}$ & $\begin{array}{l}\text { ATGTGGAATCACATAGCCAGGATAAGGGGCCA } \\
\text { TATAGGGACGGCCAAAGTGCAAAGCCGGCCCG } \\
\text { GGAAAACCGTAATGGCCATATGG }\end{array}$ & $\begin{array}{l}\text { BucLoc } \\
\text { mapping }\end{array}$ \\
\hline Buc_57-78_rev & $\begin{array}{l}\text { ATGTGGAATCACATAGCCAGGATACTGCATAAA } \\
\text { CTGATAAGGGGCCATATAGGGACGGCCAAAGT } \\
\text { G }\end{array}$ & $\begin{array}{l}\text { BucLoc } \\
\text { mapping }\end{array}$ \\
\hline
\end{tabular}




\begin{tabular}{|c|c|c|}
\hline Buc_31-51_fw & $\begin{array}{l}\text { ATG CCA CCA TCT CAG CCT TAT TTC ATG TAT } \\
\text { CAG TGG CCC ATG AAT CCA TAT GGC CAT TAC } \\
\text { GGT TTT }\end{array}$ & $\begin{array}{l}\text { BucLoc } \\
\text { mapping }\end{array}$ \\
\hline Buc_S33E_fw & $\begin{array}{l}\text { ATGCCA CCA GAG CAGCCTTATTTCAT GTAT } \\
\text { CAGTGGC } \\
\text { CCATGAATCCATATGGCCATTACGGTTTTCCCG } \\
\text { GGCCGGCTTTGCACTTTGGCCGTCCCTAT }\end{array}$ & $\begin{array}{l}\text { BucLoc } \\
\text { mutagenesis }\end{array}$ \\
\hline $\begin{array}{l}\text { Buc31- } \\
\text { 78_S33A_fw }\end{array}$ & \begin{tabular}{lrr} 
ATGCCA & CCA & GCT \\
CAGCCTTATTTCATGTATCAG & TGGCC \\
CATGAATCCATATGGCCATTACGGTTTTCCCGG \\
\multicolumn{2}{l}{ GCCGGCTTTGCACTTTGGCCGTCCCTAT }
\end{tabular} & $\begin{array}{l}\text { BucLoc } \\
\text { mutagenesis }\end{array}$ \\
\hline $\begin{array}{l}\text { Buc31- } \\
\text { 78_allYtoE_fw }\end{array}$ & $\begin{array}{l}\text { ATGCCACCATCTCAGCCTGAATTCATGGAACAG } \\
\text { TGGCCCATGAATCCAGAAGGCCATGAAGGTTTT } \\
\text { CCCGGGCCG GCTTTGCACTTTGGCCGTCCCGAA }\end{array}$ & $\begin{array}{l}\text { BucLoc } \\
\text { mutagenesis }\end{array}$ \\
\hline $\begin{array}{l}\text { Buc31- } \\
\text { 78_allYtoE_rev }\end{array}$ & $\begin{array}{l}\text { ATGTGGAATCACTTCGCCAGGTTCCTGCATAAA } \\
\text { CTGTTCAGGGGCCATTTCG } \\
\text { GGACGGCCAAAGTGCAA }\end{array}$ & $\begin{array}{l}\text { BucLoc } \\
\text { mutagenesis }\end{array}$ \\
\hline $\begin{array}{l}\text { Buc31- } \\
\text { 78_allYtoF_fw }\end{array}$ & $\begin{array}{l}\text { ATGCCACCATCTCAGCCTTTTTTCATGTTTCAGT } \\
\text { GGCCCATGAATCCATTTGGCCATTTTGGTTTTCC } \\
\text { CGGGCCGG CTTTGCACTTTGGCCGTCCCTTT }\end{array}$ & $\begin{array}{l}\text { BucLoc } \\
\text { mutagenesis }\end{array}$ \\
\hline $\begin{array}{l}\text { Buc31- } \\
\text { 78_allYtoF_rev }\end{array}$ & $\begin{array}{l}\text { ATGTGGAATCACAAAGCCAGGAAACTGCATAA } \\
\text { ACTGAAAAGGGGCCATAAAGGGACGGCCAAAG } \\
\text { TGCAA }\end{array}$ & $\begin{array}{l}\text { BucLoc } \\
\text { mutagenesis }\end{array}$ \\
\hline
\end{tabular}




\subsection{Zebrafish transgenic lines}

\subsubsection{Transgenic buc-gfp line}

To be able to isolate Buc interactors in vivo, a transgenic line was generated in the mutant Buc ${ }^{\text {p106 }}$ background (Riemer et al., 2015). For this, a copy of buc genomic locus was isolated and then a $g f p$ was inserted in frame at the 3 'end of the buc ORF. Then the buc-gfp construct was integrated into zebrafish genome using the Tol 2 transposon system. The integration rescued buc phenotype by expression of Buc-GFP fusion protein. Buc-GFP is visible starting from stage I oocytes. Furthermore, the localization of Buc-GFP can be followed through embryogenesis and at $2 \mathrm{dpf}$ the protein is still detected in living germ cells. The germ cell identity at $2 \mathrm{dpf}$ is confirmed by presence of Vasa protein. Transgenic Buc-GFP is expressed at endogenous levels, as it was shown by comparison to endogenous Buc levels by immunohistochemistry experiments. Therefore, the transgenic Buc-GFP is expected to preserve the same protein interactors as endogenous Buc.

\subsection{Molecular biology methods}

\subsubsection{High-fidelity PCR}

A standard PCR reaction was performed to amplify DNA fragments (Mullis et al., 1986). 100 ng DNA was used as template in a $50 \mu \mathrm{l}$ reaction containing 5x Phusion High Fidelity buffer (New England BioLabs, Ipswich, USA), $0.4 \mu \mathrm{M}$ of each primer (

Table 6: Setting for a standard PCR) (Eurofins, Ebersberg, Germany), $0.2 \mathrm{mM}$ of each dNTP (Thermo Scientific, Wilmington, USA) and $1 \mu \mathrm{l}$ Phusion polymerase (5 U/ $\mu 1$, homemade). TPersonal or TProfessional TRIO thermocycler (Biometra, Göttingen) were used to run the PCR. 
Table 6: Setting for a standard PCR

\begin{tabular}{l|l|l|l} 
Step & Temperature & Time & $\begin{array}{l}\text { Number } \\
\text { of cycles }\end{array}$ \\
\hline Initial denaturation & 98 & $30 \mathrm{~s}$ & \\
Denaturation & 98 & $10 \mathrm{~s}$ & \\
Primer annealing & Primer specific & $15 \mathrm{~s}$ & \\
Elongation & 72 & $40 \mathrm{~s} / \mathrm{kb}$ & $30-35 \mathrm{x}$ \\
Final elongation & 72 & $5 \mathrm{~min}$ & \\
& & &
\end{tabular}

\subsubsection{Colony PCR}

Colonies were picked with a $10 \mathrm{ul}$ pipette tip and briefly dipped into $100 \mathrm{ul} \mathrm{LB}$ medium containing an antibiotic. $1 \mathrm{ul}$ of the bacterial suspension were added to 10ul reaction containing 5x Phusion High Fidelity buffer (New England BioLabs, Ipswich, USA), $0.08 \mu \mathrm{M}$ of each primer (

Table 6: Setting for a standard PCR), $0.04 \mathrm{mM}$ of each dNTP (Thermo Scientific, Wilmington, USA) and $0.2 \mathrm{U}$ of Phusion polymerase (5 $\mathrm{U} / \mu \mathrm{l}$, homemade). TPersonal or TProfessional TRIO thermocycler (Biometra, Göttingen) were used to run the PCR. The number of amplification cycles did not exceed the number of 30 . 


\subsubsection{Agarose gel electrophoresis}

Agarose ((\% w/v) final concentration) was dissolved in $1 \mathrm{x}$ TBE buffer $(0.1 \mathrm{M}$ Tris-HCl, $0.1 \mathrm{M}$ boric acid, $5.1 \mathrm{~mm}$ EDTA, $\mathrm{pH} \mathrm{8.4)}$ and cooked in a microwave for two minutes. $0.5 \mu \mathrm{g} / \mathrm{ml}$ ethidium bromide was added to a cooled down agarose-TBE solution to visualize nucleic acids. Gel Loading Buffer II (Thermo Fisher Scientific, Waltham, USA) was added to a DNA sample in ratio 1:10, prior to loading on an agarose gel. $1 \mu \mathrm{l}$ of $1 \mathrm{~kb}$ Plus DNA Ladder (ThermoFisher, Carlsbad, USA) was loaded and run on gel in parallel with a DNA sample of interest to determine the size of DNA/RNA fragments. The electrophoresis was performed at 80-100 V for 30 minutes in 1x TBE buffer. After electrophoresis, the gels were examined using a Chemidoc gel documentation system (BioRad, Hercules, USA).

\subsubsection{Purification of DNA}

DNA was purified from agarose gel with the NucleoSpin R Gel and PCR Clean-up Kit (MachereyNagel, Düren, Deutschland). Depart from manufacturer's protocol, the column (open lid) was incubated at $44^{\circ} \mathrm{C}$ for $3 \mathrm{~min}$ after the second wash to remove residual ethanol. Then, $15 \mu \mathrm{dH} 2 \mathrm{O}$ were applied to the column and again incubated at $44^{\circ} \mathrm{C}$ for $3 \mathrm{~min}$. Finally, the column was centrifuged at $120.000 \mathrm{rpm}$ for $1 \mathrm{~min}$. Purified PCR products were stored at $-20^{\circ} \mathrm{C}$.

\subsubsection{Restriction enzyme digestion}

DNA was digested with restriction endonucleases according to the manufacturer's instructions (Thermo Scientific, Wilmington, USA). For this, $5 \mu \mathrm{g}$ of the plasmid were diluted in $17 \mathrm{uL} \mathrm{dH} 2 \mathrm{O}$ and $1 \mu \mathrm{l}$ of the restriction enzyme was added along with $2 \mathrm{uL}$ of 10x FastDigest buffer (supplied with the enzyme). This was followed up by the incubation at $37^{\circ} \mathrm{C}$ for 3 hours. Then the linearized plasmid was purified (Chapter 5.3.3). $1 \mu \mathrm{l}$ of the purified linearized plasmid was loaded on a $0.5 \%$ agarose gel along with an undigested plasmid to check for a successful linearization.

\subsubsection{Ligation of DNA}

For site-directed mutagenesis, amplified vectors were ligated using the T4 ligase. $1 \mu$ of T4 DNA ligase, $1 \mu \mathrm{l}$ of T4 DNA ligase buffer and $1 \mu \mathrm{l}$ of $100 \mathrm{mM}$ dATP (Thermo fisher, Wilmington, USA) were added to a $10 \mu$ ligation reaction. 2 different ligation reactions were performed with vector DNA and insert DNA ratios of 1:3 and 1:5, using total amount of $50 \mathrm{ng}$ DNA for each reaction. The reaction was incubated 1 hour at $37^{\circ} \mathrm{C}$ and after $1 \mu$ of the reaction was transformed into bacteria (Chapter 5.3.7).

\subsubsection{Gateway cloning}

We first inserted PCR products of interest into the pDONR221 (Entry clones) via the BP clonase induced recombination. For this, the DNA was amplified with primers containing the the attB sites (Table 4), which become att $\mathrm{L}$ sites after the recombination reaction. During the same recombination reaction, the lethal $c c d \mathrm{~B}$ gene is replaced by the gene of interest and is cut out of the target vector. As a result, only bacteria cells transformed with the recombined vector are viable (negative selection). Subsequently, the entry clones were used for subcloning to create eGFP or mCherry fusion of a 
protein of interest. The subcloning step requires the LR clonase, which will recombine the insert flanking $a t t \mathrm{~L}$ sites with the the at $\mathrm{R}$ sites of a destination vector. In order to create fusion proteins, p3eEGFP or p3emCherry vector are used in combination with the donor vector of interest (Table 3) in a multisite LR reaction to create an expression clone. This expression clone can be used for invitro transcription from the SP6 promoter (Chapter 5.3.10)

For the BP or LR reaction following reagents (Table 7, Table 8) were mixed in a $1.5 \mathrm{ml}$ reaction tube and incubated overnight at $25{ }^{\circ} \mathrm{C}$. Then, $2 \mathrm{ml}$ of Proteinase $\mathrm{K}$ solution $(2 \mathrm{mg} / \mathrm{ml})$ were added to the $\mathrm{BP}$ reaction mix and then incubated at $37{ }^{\circ} \mathrm{C}$ for 10 minutes. Subsequently, $1 \mu \mathrm{l}$ of the BP or LR reaction mix was used for transformation.

Table 7: Gateway BP reaction setup

\begin{tabular}{l|c} 
& Volume $(\mu \mathrm{l})$ \\
\hline attB-PCR product $(70-110 \mathrm{ng} / \mathrm{ml})$ & 3 \\
pDONR $^{\mathrm{TM}} 221$ vector $(100 \mathrm{ng} / \mathrm{ml})$ & 1.5 \\
$5 x$ BP ClonaseTM reaction buffer & 2.0 \\
TE buffer & 3.5 \\
\hline Total volume & 10
\end{tabular}

Table 8: Gateway LR reaction setup

\begin{tabular}{l|l|l} 
& Volume $(\mu \mathrm{l})$ & Final concentration $(\mathrm{fm})$ \\
\hline pDONR221-insert & 0.50 & 10 \\
destination vector $(\mathrm{pCS} 2+; 50 \mathrm{ng} / \mathrm{ml})$ & 0.50 & 20 \\
$\mathrm{p} 3 \mathrm{E}$ EGFP or mCherry $(50 \mathrm{ng} / \mathrm{ml})$ & 0.25 & 10 \\
$5 \mathrm{x}$ LR ClonaseTM reaction buffer & 1.00 & - \\
TE buffer (pH 8.0) & up to 5 & \\
& & \\
\hline
\end{tabular}




\subsubsection{In-fusion cloning}

The In-fusion ${ }^{\circledR}$ enzyme fuses PCR-generated sequences with a linearized vector by homologous recombination. The primers used in the PCR amplification (Table 3 ) have a 15-bp overlapping region with the DNA flanking the linearized vector. A digested vector was mixed with the insert in a 1:3 ratio along with $2 \mu \mathrm{l}$ of the $5 \mathrm{X}$ In-Fusion HD Enzyme Premix (Clonetech, Kusatsu, Japan) and $4 \mu 1$ of a nuclease-free water. The reaction was incubated for $15 \mathrm{mins}$ at $50^{\circ} \mathrm{C}$ using a Thermomixer Compact (Eppendorf, Hamburg, Germany). Subsequently, $1 \mu 1$ of the reaction was transformed in competent E.coli cells.

\subsubsection{Chemical transformation}

After thawing the chemical competent E.coli $\mathrm{dH} 5 \alpha$ cells (50ul, homemade) on ice for approximately $30 \mathrm{~min}, 1 \mathrm{ul}$ of a cloning reaction was added to cells. This was followed up by an incubation of the cells on ice for $30 \mathrm{~min}$. After incubation, the cells were heatshocked at $42{ }^{\circ} \mathrm{C}$ for $40 \mathrm{~s}$ and incubated on ice for $5 \mathrm{~min}$. Then, $250 \mathrm{ul}$ S.O.C. medium (Thermo Scientific, Wilmington, USA) was added and the cells were incubated at $180 \mathrm{rpm}$ (horizontal shaking) at $37{ }^{\circ} \mathbf{C}$ for $45 \mathrm{~min}$. The entire cell-medium solution was spread out on LB medium $(0.5 \%$ (w/v) NaCl, $0.5 \%(w / v)$ Yeast-Extract, $0.1 \%(w / v)$ Glucose; $\mathrm{pH}$ 7.0) agar plates containing the respective antibiotics. The plates were incubated overnight at $37^{\circ} \mathrm{C}$.

\subsubsection{Plasmid DNA preparation}

To amplify DNA for a midiprep culture, $100 \mathrm{ml}$ LB medium were mixed with respective antibiotics and inoculated with $100 \mathrm{uL}$ of a miniprep culture or a pippett tip dipped into a bacterial colony. The midi culture was incubated overnight at $37^{\circ} \mathrm{C}$ and $180 \mathrm{rpm}$. The next day, $50 \mathrm{ml}$ midi culture were transferred into a $50 \mathrm{ml}$ plastic tube and centrifuged at $4000 \mathrm{rpm}$ and $4^{\circ} \mathrm{C}$ for $20 \mathrm{~min}$. The supernatant was discarded and the plasmid was purified from the pellet according to the manufacturer's instructions of the NucleoBond R Xtra Midi Kit (Macherey-Nagel, Düren, Germany). The DNA was quantified with the NanoDrop 2000c spectrophotometer (Thermo Scientific, Wilmington, USA). Purified plasmid DNA was stored at $-20^{\circ} \mathrm{C}$.

\subsubsection{DNA sequencing analysis}

$1200 \mathrm{ng}$ plasmid DNA were dissolved in $12 \mathrm{uL}$ nuclease-free water in a micro tube $1.5 \mathrm{~mL}$ (Sarstedt, Nümbrecht, Germany). Subsequently, $5 \mathrm{uL}$ of $8 \mathrm{mM}$ sequencing primer (Table 9) was added to the sequencing tube. Sanger sequencing was performed by Microsynth SeqLab (Göttingen) and sequences were analyzed with the online Basic Local Alignment Search Tool (National Center for Biotechnology Information, Rockville, USA).

Table 9: Primers used for sequencing

Sequence (5' to 3') 


\begin{tabular}{l|l} 
Name & \\
\hline SP6 & ATTTAGGTGACACTATAGAA \\
eGFP-end_fw & ACATGGTCCTGCTGGAGTTC \\
GFP_begin_rv & CTTCATGTGGTCGGGGTAGC \\
GFPfor & CACAAGTTCAGCGTGTCCGG \\
GFPrev & CAAAGACCCCAACGAGAAGC
\end{tabular}

\subsubsection{In vitro transcription}

In order to prepare a DNA plasmid for transcription, it was linearized (Section 5.3.4) by NotI enzyme (Thermo Fisher Scientific, Waltham, USA). Next, in-vitro transcription was performed using the mMESSAGE mMACHINETM SP6 Transcription Kit (Thermo Scientific, Waltham, USA). Next, the sense RNA was purified by spin-column chromatography (Illustra ProbeQuant G-50 Micro Columns, GE Healthcare Life Sciences, Chicago, USA). The concentration of the purified RNA was measured at the NanoDrop 2000c spectrophotometer (Thermo Scientific, Wilmington, USA) and verification of fragment length was performed by agarose gel electrophoresis. RNA was aliquoted and stored at $20^{\circ} \mathrm{C}$.

\subsection{Biochemical methods}

\subsubsection{SDS-polyacrylamide gel electrophoresis}

SDS-PAGE is used to separate proteins corresponding to their mass/charge ratio (Laemli, 1970). Input and Co-IP samples from embryo lysates or in-vitro translated proteins were boiled in the $2 \mathrm{X}$ SDS loading buffer (Merck, Kenilworth, New Jersey, USA) at $95{ }^{\circ} \mathrm{C}$ for 5 mins before loading onto a $10 \%$ Acrylamide gel. 2 ul of the PageRuler ${ }^{\mathrm{TM}}$ Plus Prestained protein ladder marker (Thermo Scientific, Waltham, USA) was loaded onto a gel as a protein standard to determine the molecular weight of protein bands. The gel was run vertically in 1X SDS Running buffer (25 mM Tris, $250 \mathrm{mM}$ glycine, $0.01 \%$ SDS) at a constant voltage of $80 \mathrm{~V}$. Then after the dye font reached the resolving gel, the voltage was raised to $120 \mathrm{~V}$.

\subsubsection{Colloidal Coomassie staining}

Gels were stained with a self-made colloidal coomassie $(0.08 \%$ (w/v) Coomassie Brilliant Blue G250 solution, $1.6 \%$ (v/v) ortho-Phosphoric acid, 8\% (w/v) ammonium sulfate, $20 \%$ methanol) (Neuhoff, 1988)for 6 hours or overnight and then destained using a destaining solution (40\% methanol, $10 \%$ glacial acetic acid) overnight at room temperature. Gels were imaged using a standard scanner (EPSON). 


\subsubsection{Western blot}

To detect proteins from embryo lysates or in-vitro translation, proteins were subjected to wet transfer and transferred to a nitrocellulose membrane (Burnette, 1981). The transfer was performed at $4{ }^{\circ} \mathrm{C}$, at $80 \mathrm{~V}$ for 90 mins, using Trans-Blot ${ }^{\circledR}$ (Bio-rad, Hercules, USA) cell filled with wet transfer buffer (25 mM Tris, $190 \mathrm{mM}$ glycine, 30\% methanol). After the transfer, the membrane was incubated with a primary antibody dilution (Table 10) overnight at $4{ }^{\circ} \mathrm{C}$, in $5 \%$ milk powder in 1x Tris-buffered saline (TBS) withTween20, TBST (20 mM Tris, $150 \mathrm{mM} \mathrm{NaCl}, 0.05 \%$ Tween20, $\mathrm{pH}$ 7.4-7.6). Then, the membrane was washed 3x 10 min with TBST and incubated 1.5 hours with a dilution of the secondary antibody (Table 10) at RT in dark. Then, the membrane was washed again 3x 10 min with TBST. Finally, the membrane was imaged using the Odyssey® CLx Infrared Imaging system (LiCor) and processed using the Image Studio ${ }^{\text {TM }}$ software (Li-Cor).

\subsubsection{Co-immunoprecipitation}

For protein Co-IP from zebrafish, 200-1000 embryos were dechorionated (Chapter 5.1.3) and deyolked (Chapter 5.1.4). Then embryos were lysed (100 ul lysis buffer (Table 10) / 200 embryos) using a pestle on ice and the lysates were incubated on ice for $30 \mathrm{~min}$. Each $10 \mathrm{~min}$ the lysates were pipetted extensively using a $200 \mathrm{ul}$ pipette tip. In parallel, GFP-Trap Magnetic Agarose (MA) beads (Chromotek, Planegg, Germany) (10 ul / 200 embryos) were equilibrated with the dilution buffer (Table 11 Co-ip and lysis buffers), as stated by manufacturer. Then the lysates were added to the beads in the dilution buffer, to the final volume of $500 \mathrm{ul}$. This was followed by $30 \mathrm{~min}$ incubation at $4^{\circ} \mathrm{C}$ on rotation wheel. Afterward, the incubated beads were washed 3 times with dilution buffer, as stated by manufacturer. In the end, proteins were eluted by mixing $20 \mathrm{ul}$ of the dilution buffer with $20 \mu 1$ of the 2X SDS loading buffer and boiled $10 \mathrm{~min}$ at $95^{\circ} \mathrm{C}$ (Merck, Kenilworth, USA).

Table 10: Antibodies used for western blotting

\begin{tabular}{l|l} 
Antibody & Dilution \\
\hline guinea pig- $\alpha$-Buc (BioGenes, Berlin) & $1: 5000$ \\
Mouse- $\alpha$-GFP (Merck, Kenilworth, USA) & $1: 2500$ \\
Mouse- $\alpha$ - $\alpha$-Tubulin (Merck, Kenilworth, USA) & $1: 20000$ \\
Anti-Vasa (gifted by Prof. Dr. Knaut; (Knaut et al., 2000)) & $1: 2500$ \\
goat- $\alpha$-guinea pig 800CW (IRDye, Li-Cor) & $1: 20000$ \\
goat- $\alpha$-mouse 680CW(IRDye, Li-Cor) & $1: 20000$
\end{tabular}


Table 11 Co-ip and lysis buffers

\begin{tabular}{l|l|l} 
Reagent & Lysis buffer & Dilution buffer \\
\hline Tris-HCl (pH7.5) & $10 \mathrm{mM}$ & $10 \mathrm{mM}$ \\
$\mathrm{NaCl}$ & $150 \mathrm{mM}$ & - \\
EDTA & $1 \mathrm{mM}$ & - \\
NP40 100\% Protease & $0.5 \%$ & - \\
EDTA-free & $1 / 25$ & \\
$\begin{array}{l}\text { Inhibitor Cocktail (1 tablet } \\
\text { dissolved in 1 ml H20) } \\
\text { (Roche, Basel, Switzerland) }\end{array}$ & \\
$\begin{array}{l}\text { Phenylmethylsulfonylfluorid } \\
\text { Merck, Kenilworth, USA). }\end{array}$ & $1 \mathrm{mM}$ & - \\
$\begin{array}{l}\text { Soybean Trypsin Inhibitor } \\
\text { (Merck, Kenilworth, USA) }\end{array}$ & $0.1 \mathrm{mg} / 1 \mathrm{ml}$ \\
$\begin{array}{l}\text { Phosphatase inhibitor } \\
\text { cocktail I Abcam, } \\
\text { Cambridge, UK) }\end{array}$ & $1 / 50$ & - \\
\end{tabular}

\subsubsection{Fixation of zebrafish oocytes}

Zebrafish oocytes were fixated for subsequent immunostaining. A female fish was sacrificed to collect the oocytes. The ovaries were dissected and placed in a Petri dish containing 1x Phosphatebuffered Saline (PBS, NaCl 137 mM, KCl 2.7 mM, Na $\mathrm{HPO}_{4} 10 \mathrm{mM}, \mathrm{KH}_{2} \mathrm{PO}_{4} 1.8 \mathrm{mM}$ ). Ovaries were dissociated for $3 \mathrm{~min}$ at room temperature in Proteinase K solution (0.1 M Tris ( $\mathrm{pH} 7.5), 10 \mathrm{mM}$ EDTA, $50 \mu \mathrm{g} / \mathrm{ml}$ Proteinase K) (Merck, Darmstadt, Germany). Afterwards, the oocytes were washed twice with PBS and subsequently fixated overnight in $4 \%$ paraformaldehyde (PFA). Ovaries were directly used for immunostaining. To visualize actin and microtubule cytoskeleton, embryos were fixated in glyoxal solution ( $\mathrm{pH} 5$, as described in Richter et al., 2018) overnight at $4{ }^{\circ} \mathrm{C}$. Subsequently, embryos were quenched with $100 \mathrm{mM} \mathrm{NH}{ }_{4} \mathrm{Cl}$ solution at $\mathrm{RT}$ and directly used for immunohistochemistry.

\subsubsection{Fixation of zebrafish embryos}

Embryos of a required stage were collected, dechorionated (Section 5.1.3) and fixated overnight at 4 ${ }^{\circ} \mathrm{C}$ with $4 \%$ PFA. Then, embryos were washed 3 times $10 \mathrm{~min}$ in PBS and directly used for immunohistochemistry. 


\subsubsection{Immunostaining of PFA fixated embryos and oocytes}

To visualize endogenous proteins after the fixation with PFA, oocytes and embryos were immunostained. For reasons of legibility only embryos will be named in the following. Embryos were distributed in a 24-well plate and PBS $0.5 \%$ Triton X-100 solution was added to permeabilize embryos for 30 min at RT while rocking. Then, this solution was replaced by a blocking solution (PBS 0.1\% Triton X-100, 0.1\% Tween20, 5\% Bovine Serum Albumin (BSA), 10\% Goat serum) and rocked 2 hrs at RT. Then, dilution of a primary antibody in the blocking solution was added to embryos and incubated overnight at $4{ }^{\circ} \mathrm{C}$. Then, the primary antibody (

Table 12) solution was replaced a washing solution (PBS 0.1\% Tween20 (PBT)) to rinse embryos 3 times for $30 \mathrm{~min}$. Then, dilution of a secondary fluorescently labelled antibody in PBT (

Table 12) was added to embryos and incubated overnight at $4{ }^{\circ} \mathrm{C}$. Afterwards, embryos were washed 3 times with the wash solution for 30 mins and incubated with DAPI $(0.8 \mathrm{ug} / \mathrm{ml})$ diluted in PBT. Then, embryos were washed again 3 times with the wash solution for $30 \mathrm{~min}$. After, the PBT was replaced by 30:70 PBT:glycerol solution and incubated 1 hour at RT. Then the 30:70 glycerol:PBT solution was replaced by the 20:80 PBT:glycerol solution and incubated 1 hour at RT. Afterwards, a plastic grid was placed into a fluorodish (Fluorodish $35 \mathrm{~mm}$; WPI, Sarasota, USA) and embryos were positioned in the animal orientation. Then, the excess glycerol:PBT was removed from the fluorodish by a Pasteur pipette and embryos were analyzed by confocal microscope (Carl Zeiss Microscopy, Jena). Subsequently, images were analyzed using the ZEN 2011 software (Carl Zeiss Microscopy, Jena).

\subsubsection{Immunostaining of glyoxal fixated embryos and oocytes}

To visualize endogenous actin and microtubules after the fixation with glyoxal, oocytes and embryos were immunostained. For reasons of legibility only embryos will be named in the following. To visualize actin, quenched embryos were washed 3 times with PBS $0.1 \%$ Tween20 for 15 mins and permeabilized with PBS $0.2 \%$ Triton-X 100 for $2 \mathrm{hrs}$ at RT. Then embryos were incubated with the working solution of Phalloidin 488 (Table 12) diluted 1:20 in PBS Triton-X 100 overnight at $4{ }^{\circ} \mathrm{C}$. Then embryos were dehydrated with PBS:glycerol series (70:30, 60:40, 80:20) and proceeded for imaging with confocal microscopy (5.4.7).

To visualize microtubules, quenched embryos were blocked with PBS, $0.1 \%$ Triton X-100, $1 \%$ BSA solution $4 \mathrm{hrs}$ at RT. Then the blocking solution was replaced by the primary antibody solution of $\alpha$ $\alpha$-tubulin (Table 12: Antibodies and other reagents used for immunostaining) and incubated overnight at $4{ }^{\circ} \mathrm{C}$. Then, the primary antibody was recognized using an Alexa 488-conjugated rabbit anti-mouse antibody (Table 12: Antibodies and other reagents used for immunostaining) in PBS, $1 \%$ BSA, $0.1 \%$ Triton X100. Then embryos were dehydrated with PBS:glycerol series (70:30, 60:40, 80:20) and proceeded for imaging with confocal microscopy (5.4.7). 
Table 12: Antibodies and other reagents used for immunostaining

\begin{tabular}{|c|c|}
\hline Antibodies and other reagents & Dilution \\
\hline guinea pig- $\alpha$-Buc (Biogenes, Berlin) & $1: 5000$ \\
\hline rabbit- $\alpha-p-N M I I$ (Cell Signaling Technology, Danvers, USA) & $1: 50$ \\
\hline GFP-booster Atto 488 (ChromoTek, Planegg-Martinsried) & $1: 500$ \\
\hline Rat- $\alpha-Z O 1$ (Santa Cruz, Dallas, Texas, USA) & $1: 25$ \\
\hline Mouse- $\alpha-Z O 1$ (Thermo Scientific, Waltham, Massachusetts, USA) & $1: 1000$ \\
\hline Mouse- $\alpha-Z O 2$ (Thermo Scientific, Waltham, Massachusetts, USA) & $1: 50$ \\
\hline Mouse- $\alpha$-E-cadherin (BD Transduction Laboratories, Franklin Lakes, New Jersey, & $1: 50$ \\
\hline U.S.A) & \\
\hline Mouse- $\alpha$-afadin (Thermo Scientific, Waltham, Massachusetts, USA) & $1: 50$ \\
\hline Mouse- $\alpha$-integrin- $\alpha$-v 5 (Abcam, Cambridge, UK) & $1: 50$ \\
\hline Mouse- $\alpha$-Kif23 (GeneTex, Irvine, California, USA) & $1: 50$ \\
\hline Mouse- $\alpha-J A M-A$ (Santa Cruz, Dallas, Texas, USA) & $1: 50$ \\
\hline Mouse- $\alpha$ - $\alpha$-Tubulin (Merck, Kenilworth, USA) & $1: 2500$ \\
\hline Phalloidin 488 (Cambrex, East Rutherford, New Jersey, USA) & $6.6 \mu \mathrm{M}$ \\
\hline Mouse- $\alpha$ - pTyr (Cell signalling, Danvers, Massachusetts, USA) & $1: 50$ \\
\hline rabbit- $\alpha$-Vasa (gifted by Prof. Dr. Knaut; (Knaut et al., 2000)) & $1: 500$ \\
\hline Mouse- $\alpha-\beta$-Catenin (Merck, Kenilworth, USA) & $1: 1000$ \\
\hline goat- $\alpha$-guinea pig Alexa Fluor 488 (Life Technologies, Carlsbad, USA) & $1: 500$ \\
\hline goat- $\alpha$-rabbit, mouse or rat Alexa Fluor 594 (Life Technologies, Carlsbad, USA) & $1: 500$ \\
\hline
\end{tabular}




\subsubsection{Protein in-vitro translation}

Proteins were synthesized with the TnT SP6 Quick Coupled Transcription/Translation System (Promega, Madison, Wisconsin, USA).

\subsubsection{Protein aggregation assay}

HEK cells were seeded $10^{4}$ cells /per well in an eight-chambered slide (Sarstedt) and transfected with $100 \mathrm{ng}$ of a plasmid combined with the ScreenFectA reagent. Cells were imaged after 48 hours using 10X objective with a 10X digital zoom with an LSM780 confocal microscope and cell profiles were analyzed with ZEN2011 software (Carl Zeiss Microscopy, Göttingen).

\subsubsection{Phosphatase treatment}

Embryo lysates were incubated with Lambda Protein Phosphatase (NEB, Ipswich, Massachusetts, USA) as stated by the manufacturer.

\subsection{Bioinformatics methods}

\subsubsection{Pairwise sequence alignment}

Protein sequences were pairwise aligned by the Needleman-Wunsch algorithm with the EMBL-EBI alignment software EMBOSS Needle (McWilliam et al., 2013). Standard settings were applied. Zebrafish Bucky ball (H0WFA5), Buc2l (R4GEN3) and Velo1 (F6VL84) sequences have been used for the alignments.

\subsubsection{Aggregation propensity prediction}

Bucky ball protein sequence (H0WFA5) was analyzed using the web-based aggregation prediction program PLAAC (Lancaster et al., 2014, http://plaac.wi.mit.edu/)

\subsubsection{Prediction of prion-like domains}

The following web-based algorhytms (Table 13) were used to predict the exact position of prion-like domains within the localization signal of Bucky ball (H0WFA5)(residues 11-88).

Table 13: The algorithms used for the prion-like domains prediction in Bucky ball

\begin{tabular}{|c|c|}
\hline Program & Reference \\
\hline Fold amyloid (http://bioinfo.protres.ru/fold-amyloid/) & $\begin{array}{ll}\text { (Fernandez-Escamilla, } & \text { Rousseau, } \\
\text { Schymkowitz, \& Serrano, 2004) } & \end{array}$ \\
\hline $\begin{array}{l}\text { APPNN (https://omictools.com/appnn-tool) } \\
\text { FISH amyloid (https://omictools.com/fish-amyloid-tool) }\end{array}$ & $\begin{array}{l}\text { (Família, Dennison, Quintas, \& Phoenix, } \\
\text { 2015) }\end{array}$ \\
\hline Aggrescan (http://bioinf.uab.es/aggrescan/) & $\begin{array}{l}\text { (Gasior \& Kotulska, 2014) } \\
\text { (Conchillo-Solé et al., 2007) }\end{array}$ \\
\hline
\end{tabular}




\subsubsection{Prediction of intrinsic protein disorder}

The prediction of the intrinsically disordered regions was performed as described in Krishnakumar et al., 2018.

\subsubsection{Fluorescence quantification}

FIJI (Schindelin et al., 2012) was used for the quantification of relative fluorescence in live-imaged embryos.

\subsubsection{Processing of mass spectrometry data}

Raw mass spectrometry data was processed using Microsoft Excel.

\subsection{Statistical methods}

All statistical analysis of experimental data was performed in Microsoft Excel as indicated in figure legends.

\section{References}

Albamonte, M. I., Albamonte, M. S., Stella, I., Zuccardi, L., \& Vitullo, A. D. (2013). The infant and pubertal human ovary: Balbiani's body-associated VASA expression, immunohistochemical detection of apoptosis-related BCL2 and BAX proteins, and DNA fragmentation. Human Reproduction, Vol.28, pp. 698-706.

Alberti, S. (2017). The wisdom of crowds: Regulating cell function through condensed states of living matter. Journal of Cell Science, Vol. 130, pp. 2789-2796.

Alberti, S., Gladfelter, A., \& Mittag, T. (2019). Considerations and Challenges in Studying LiquidLiquid Phase Separation and Biomolecular Condensates. Cell, Vol. 176, pp. 419-434.

Anderson, J. M., Balda, M. S., \& Fanning, A. S. (1993). The structure and regulation of tight junctions. Current Opinion in Cell Biology, Vol.5, pp. 772-778

Anderson, J. M., Van Itallie, C. M., \& Fanning, A. S. (2004, April). Setting up a selective barrier at the apical junction complex. Current Opinion in Cell Biology, Vol. 16, pp. 140-145.

Bateman, A. (2019). UniProt: A worldwide hub of protein knowledge. Nucleic Acids Research, 47(D1), D506-D515.

Berchowitz, L. E., Kabachinski, G., Walker, M. R., Carlile, T. M., Gilbert, W. V., Schwartz, T. U., \& Amon, A. (2015). Regulated Formation of an Amyloid-like Translational Repressor Governs Gametogenesis. Cell, Vol.163(2), 406-418.

Beutel, O., Maraspini, R., Pombo-Garcia, K., Martin-Lemaitre, C., \& Honigmann, A. (2019). Phase separation of zonula occludens proteins drives formation of tight junctions, bioRxiv preprint

Boke, E., Ruer, M., Wühr, M., Coughlin, M., Lemaitre, R., Gygi, S. P., ... Mitchison, T. J. (2016). Amyloid-like Self-Assembly of a Cellular Compartment. Cell, Vol.166(3), 637-650 
Bontems, F., Stein, A., Marlow, F., Lyautey, J., Gupta, T., Mullins, M. C., \& Dosch, R. (2009). Bucky Ball Organizes Germ Plasm Assembly in Zebrafish. Current Biology, Vol.19(5), 414-422

Braat, A. K., Van De Water, S., Goos, H., Bogerd, J., \& Zivkovic, D. (2000). Vasa protein expression and localization in the zebrafish. Mechanisms of Development, Vol.95, 271-274

Brangwynne, C. P., Hoege, C., Gharakhani, J., Jülicher, F., \& Hyman, A. A. (2009). Germline P Granules Are Liquid Droplets That Localize by Controlled Dissolution/Condensation, Science, Vol.324, 1729-1732

Brangwynne C. P., Mitchison T. J., Hyman A. A. (2011). Active liquid-like behavior of nucleoli determines their size and shape in Xenopus laevis oocytes. PNAS. Vol. 108(11), 4334-4339

Brizuela, B. J., Wessely, O., \& De Robertis, E. M. (2001). Overexpression of the Xenopus tightjunction protein claudin causes randomization of the left-right body axis. Developmental Biology, Vol.230(2), 217-229

Burnette, W. N. (1981). "Western Blotting": Electrophoretic transfer of proteins from sodium dodecyl sulfate-polyacrylamide gels to unmodified nitrocellulose and radiographic detection with antibody and radioiodinated protein A. Analytical Biochemistry, Vol.112(2), 195-203

Campbell, P. D., Heim, A. E., Smith, M. Z., \& Marlow, F. L. (2015). Kinesin-1 interacts with Bucky ball to form germ cells and is required to pattern the zebrafish body axis. Development, Vol.142, 2996-3008

Carpenter, K., Bell, R. B., Yunus, J., Amon, A., \& Berchowitz, L. E. (2018). PhosphorylationMediated Clearance of Amyloid-like Assemblies in Meiosis. Developmental Cell, Vol.45(3), 392-405

Cerdà, J., Reidenbach, S., Prätzel, S., \& Franke, W. W. (1999). Cadherin-Catenin Complexes During Zebrafish Oogenesis: Heterotypic Junctions Between Oocytes and Follicle Cells1. Biology of Reproduction, Vol.61(3), 692-704

Chen, M. C., Zhou, Y., \& Detrich, H. W. (2002). Zebrafish mitotic kinesin-like protein 1 (Mklp1) functions in embryonic cytokinesis. Physiological Genomics, Vol.8, 51-66

Claußen, M., \& Pieler, T. (2004). Xvelo1 uses a novel 75-nucleotide signal sequence that drives vegetal localization along the late pathway in Xenopus oocytes. Developmental Biology, Vol.266, 270-284

Cohen, P. T., \& Cohen, P. (1989). Discovery of a protein phosphatase activity encoded in the genome of bacteriophage lambda. Probable identity with open reading frame 221. The Biochemical Journal, Vol.260(3), 931-934

Conchillo-Solé, O., de Groot, N. S., Avilés, F. X., Vendrell, J., Daura, X., \& Ventura, S. (2007). AGGRESCAN: A server for the prediction and evaluation of "hot spots" of aggregation in polypeptides. BMC Bioinformatics, Vol.8

Cox, R. T. (2003). A Balbiani body and the fusome mediate mitochondrial inheritance during Drosophila oogenesis. Development, Vol.130, 1579-1590 
Danilchik, M. V., Funk, W. C., Brown, E. E., \& Larkin, K. (1998). Requirement for microtubules in new membrane formation during cytokinesis of Xenopus embryos. Developmental Biology, 194(1), 47-60

Davey, N. E., Van Roey, K., Weatheritt, R. J., Toedt, G., Uyar, B., Altenberg, B., ... Gibson, T. J. (2012). Attributes of short linear motifs. Molecular BioSystems, Vol. 8(1)

DeHaan, H., McCambridge, A., Armstrong, B., Cruse, C., Solanki, D., Trinidad, J. C., ... Gao, M. (2017). An in vivo proteomic analysis of the Me31B interactome in Drosophila germ granules, FEBS Letters, Vol. 591, 3536-3547

Dosch, R., Wagner, D. S., Mintzer, K. A., Runke, G., Wiemelt, A. P., \& Mullins, M. C. (2004). Maternal control of vertebrate development before the midblastula transition: Mutants from the zebrafish I. Developmental Cell, 6(6), 771-780

Dosch, R. (2015). Next generation mothers: Maternal control of germline development in zebrafish. Critical Reviews in Biochemistry and Molecular Biology, Vol.50(1), 54-68

Elkouby, Y. M., Jamieson-Lucy, A., \& Mullins, M. C. (2016). Oocyte Polarization Is Coupled to the Chromosomal Bouquet, a Conserved Polarized Nuclear Configuration in Meiosis. PLoS Biology,Vol.14(1)

Eno, *, 1\#, C., Hansen, C. L., Pelegri, F., \& Pelegri, F. (2019). Aggregation, segregation and dispersal of homotypic germ plasm RNPs in the early zebrafish embryo, Developmental dynamics

Eno, C., Gomez, T., Slusarski, D. C., \& Pelegri, F. (2018a). Slow calcium waves mediate furrow microtubule reorganization and germ plasm compaction in the early zebrafish embryo, Development, Vol.145

Eno, Celeste, \& Pelegri, F. (2013). Gradual recruitment and selective clearing generate germ plasm aggregates in the zebrafish embryo. Bioarchitecture, Vol.3(4), 1-6

Eno, Celeste, \& Pelegri, F. (2018b). Modulation of F-actin dynamics by maternal Mid1ip1L controls germ plasm aggregation and furrow recruitment in the zebrafish embryo, Development, Vol.145

Eno, Celeste, Solanki, B., \& Pelegri, F. (2016). aura ( mid1ip11 ) regulates the cytoskeleton at the zebrafish egg-to-embryo transition . Development, Vol.143, 1585-1599

Ephrussi, A., Dickinson, L. K., \& Lehmann, R. (1991). oskar organizes the germ plasm and directs localization of the posterior determinant nanos. Cell, Vol.66, 37-50

Ephrussi, A., \& Lehmann, R. (1992). Induction of germ cell formation by oskar. Nature, Vol. $358(6385), 387-392$

Escobar-Aguirre, M., Elkouby, Y. M., \& Mullins, M. C. (2017). Localization in oogenesis of maternal regulators of embryonic development. In Advances in Experimental Medicine and Biology, Vol.953, 173-207

Ewen-Campen, B., Schwager, E. E., \& Extavour, C. G. M. (2010, January). The molecular machinery of germ line specification. Molecular Reproduction and Development, Vol. 77, 3-18 
Extavour, C. G., \& Akam, M. (2003, December). Mechanisms of germ cell specification across the metazoans: Epigenesis and preformation. Development, Vol. 130, 5869-5884

Família, C., Dennison, S. R., Quintas, A., \& Phoenix, D. A. (2015). Prediction of peptide and protein propensity for amyloid formation. PLOS ONE, 10(8)

Fernandez-Escamilla, A. M., Rousseau, F., Schymkowitz, J., \& Serrano, L. (2004). Prediction of sequence-dependent and mutational effects on the aggregation of peptides and proteins. Nature Biotechnology, 22(10), 1302-1306

Fitzpatrick, P., Shattil, S. J., \& Ablooglu, A. J. (2014). C-terminal COOH of integrin $\beta 1$ is necessary for $\beta 1$ association with the kindlin-2 adapter protein. Journal of Biological Chemistry,

Fleming, T. P., Papenbrock, T., Fesenko, I., Hausen, P., \& Sheth, B. (2000). Assembly of tight junctions during early vertebrate development. Seminars in Cell and Developmental Biology, Vol. 11, 291-299

Furuse, M., Hirase, T., Itoh, M., Nagafuchi, A., Yonemura, S., Tsukita, S., \& Tsukita, S. (1993). Occludin: A novel integral membrane protein localizing at tight junctions. Journal of Cell Biology, Vol. 123(6 II), 1777-1788

Furuse, Mikio, Itoh, M., Hirase, T., Nagaftichi, A., Yonemura, S., Tsukita, S., \& Tsukita, S. (1994). Direct association of occludin with ZO-1 and its possible involvement in the localization of occludin at tight junctions. Journal of Cell Biology, Vol. 127(6 I), 1617-1626

Gasior, P., \& Kotulska, M. (2014). FISH Amyloid - a new method for finding amyloidogenic segments in proteins based on site specific co-occurence of aminoacids. BMC Bioinformatics, 15(1).

Giraldez, A. J., Mishima, Y., Rihel, J., Grocock, R. J., Van Dongen, S., Inoue, K., ... Schier, A. F. (2006). Zebrafish MiR-430 promotes deadenylation and clearance of maternal mRNAs. Science, Vol. 312(5770), 75-79

Guraya, S. S. (1979). Recent Advances in the Morphology, Cytochemistry, and Function of Balbiani's Vitelline Body in Animal Oocytes. International Review of Cytology, Vol. 59, 249321

Han, T. W., Kato, M., Xie, S., Wu, L. C., Mirzaei, H., Pei, J., ... McKnight, S. L. (2012). Cell-free formation of RNA granules: Bound RNAs identify features and components of cellular assemblies. Cell, Vol. 149, 768-779,

Hashimoto, Y., Maegawa, S., Nagai, T., Yamaha, E., Suzuki, H., Yasuda, K., \& Inoue, K. (2004). Localized maternal factors are required for zebrafish germ cell formation. Developmental Biology, Vol. 268, 152-161

Hashimoto, Y., Suzuki, H., Kageyama, Y., Yasuda, K., \& Inoue, K. (2006). Bruno-like protein is localized to zebrafish germ plasm during the early cleavage stages. Gene Expression Patterns, Vol. 6, 201-205

Heasman, J., Quarmby, J., \& Wylie, C. C. (1984). The mitochondrial cloud of Xenopus oocytes: The source of germinal granule material. Developmental Biology, Vol. 105(2), 458-469. 
Hennig, S., Kong, G., Mannen, T., Sadowska, A., Kobelke, S., Blythe, A., ... Fox, A. H. (2015). Prion-like domains in RNA binding proteins are essential for building subnuclear paraspeckles. Journal of Cell Biology, Vol.210(4), 529-539

Hofmann, I., Schnölzer, M., Kaufmann, I., \& Franke, W. W. (2002). Symplekin, a constitutive protein of karyo- and cytoplasmic particles involved in mRNA biogenesis in Xenopus laevis oocytes. Molecular Biology of the Cell, Vol. 13(5), 1665-1676.

Houwing, S., Kamminga, L. M., Berezikov, E., Cronembold, D., Girard, A., van den Elst, H., ... Ketting, R. F. (2007). A Role for Piwi and piRNAs in Germ Cell Maintenance and Transposon Silencing in Zebrafish. Cell, Vol. 129(1), 69-82.

Huang, J. D., Brady, S. T., Richards, B. W., Stenoien, D., Resau, J. H., Copeland, N. G., \& Jenkins, N. A. (1999). Direct interaction of microtubule- and actin-based transport motors. Nature, Vol. 397, 267-269

Huang, L., Grammatikakis, N., Yoneda, M., Banerjee, S. D., \& Toole, B. P. (2000). Molecular characterization of a novel intracellular hyaluronan-binding protein. Journal of Biological Chemistry, Vol. 275(38), 29829-29839

Jesuthasan, S. (1998), Furrow-associated microtubule arrays are required for the cohesion of zebrafish blastomeres following cytokinesis, Journal of Cell Science, Vol. 111, 3695-3703

Irie, N., \& Kuratani, S. (2014, December 15). The developmental hourglass model: A predictor of the basic body plan?. Development (Cambridge), Vol. 141, 4649-4655

Irvine, K., Stirling, R., Hume, D., \& Kennedy, D. (2004, December). Rasputin, more promiscuous than ever: A review of G3BP. International Journal of Developmental Biology, Vol. 48, 10651077

Jankovics, F., Sinka, R., Lukácsovich, T., \& Erdélyi, M. (2002). MOESIN crosslinks actin and cell membrane in Drosophila oocytes and is required for OSKAR anchoring. Current Biology, Vol. 12(23), 2060-2065

Jeske, M., Bordi, M., Glatt, S., Müller, S., Rybin, V., Müller, C. W., \& Ephrussi, A. (2015). The crystal structure of the Drosophila germline inducer Oskar identifies two domains with distinct Vasa Helicase- and RNA-binding activities. Cell Reports, 12(4), 587-598

Jeske, M., Müller, C. W., \& Ephrussi, A. (2017). The LOTUS domain is a conserved DEAD-box RNA helicase regulator essential for the recruitment of Vasa to the germ plasm and nuage. Genes and Development, Vol. 31(9), 939-952

Juliano, C. E., Swartz, S. Z., \& Wessel, G. M. (2010, December 15). A conserved germline multipotency program. Development, Vol. 137, 4113-4126

Jülich, D., Geisler, R., Holley, S. A., Van Bebber, F., Busch-Nentwich, E., Dahm, R., ... Weiler, C. (2005). Integrin $\alpha 5$ and delta/notch signaling have complementary spatiotemporal requirements during zebrafish somitogenesis. Developmental Cell, Vol. 8(4), 575-586

Laemmli, U.K., 1970. Cleavage of structural proteins during the assembly of the head of bacteriophage T4. Nature. Vol. 227, 680-685 
Kane, D. A, Warga M. R., Kimmel C. B. (1992). Mitotic domains in the early embryo of the zebrafish. Nature, Vol. 360, 735-737

Kato, M., Han, T. W., Xie, S., Shi, K., Du, X., Wu, L. C., ... McKnight, S. L. (2012). Cell-free formation of RNA granules: Low complexity sequence domains form dynamic fibers within hydrogels. Cell. Vol. 149, 753-767

Kiener, T. K., Selptsova-Friedrich, I., \& Hunziker, W. (2008). Tjp3/zo-3 is critical for epidermal barrier function in zebrafish embryos. Developmental Biology, Vol. 316(1), 36-49

Kim-Ha, J., Smith, J. L., \& Macdonald, P. M. (1991). oskar mRNA is localized to the posterior pole of the Drosophila oocyte. Cell, Vol. 66(1), 23-35

Kimmel, C. B., Ballard, W. W., Kimmel, S. R., Ullmann, B., \& Schilling, T. F. (1995). Stages of embryonic development of the zebrafish. Developmental Dynamics, Vol. 203(3), 253-310

King, M. Lou, Zhou, Y., \& Bubunenko, M. (1999). Polarizing genetic information in the egg: RNA localization in the frog oocyte. BioEssays, Vol. 21, 546-557

Kistler, K. E., Trcek, T., Hurd, T. R., Chen, R., Liang, F. X., Sall, J., ... Lehmann, R. (2018). Phase transitioned nuclear oskar promotes cell division of drosophila primordial germ cells. ELife, Vol. 7

Kloc, M., Bilinski, S., \& Etkin, L. D. (2004). The Balbiani Body and Germ Cell Determinants: 150 Years Later, Current Topics in Developmental Biology, Vol. 59

Knaut, H., Pelegri, F., Bohmann, K., Schwarz, H., \& Nüsslein-Volhard, C. (2000). Zebrafish vasa RNA but not its protein is a component of the germ plasm and segregates asymmetrically before germline specification. Journal of Cell Biology, Vol. 149(4), 875-888

Ko K. S., Pamela D. Arora, Vinay Bhide, Austin Chen and Christopher A. G. McCulloch (2001). Cell-cell adhesion in human fibroblasts requires calcium signaling, Vol. 114, 1155-1167

Kogo, N., Tazaki, A., Kashino, Y., Morichika, K., Orii, H., Mochii, M., \& Watanabe, K. (2011). Germ-line mitochondria exhibit suppressed respiratory activity to support their accurate transmission to the next generation. Developmental Biology, Vol. 349, 462-469

Köprunner, M., Thisse, C., Thisse, B., \& Raz, E. (2001). A zebrafish nanos-related gene is essential for the development of primordial germ cells. Genes and Development, Vol. 15(21), 2877-2885.

Krishnakumar, P., Riemer, S., Perera, R., Lingner, T., Goloborodko, A., Khalifa, H., ... Dosch, R. (2018). Functional equivalence of germ plasm organizers. PLoS Genetics, Vol. 14(11)

Kroschwald, S., Maharana, S., Mateju, D., Malinovska, L., Nüske, E., Poser, I., ... Alberti, S. (2015). Promiscuous interactions and protein disaggregases determine the material state of stressinducible RNP granules. ELife, Vol. 4

Kumano, G. (2015, May 1). Evolution of germline segregation processes in animal development. Development Growth and Differentiation, Vol. 57, 324-332

Lancaster, A. K., Nutter-Upham, A., Lindquist, S., \& King, O. D. (2014). PLAAC: A web and 
command-line application to identify proteins with prion-like amino acid composition. Bioinformatics, Vol. 30(17), 2501-2502

Lee, K. Y., Davies, T., \& Mishima, M. (2012, August 1). Cytokinesis microtubule organisers at a glance. Journal of Cell Science, Vol. 125, 3495-3500

Lei, L., \& Spradling, A. C. (2016). Mouse oocytes differentiate through organelle enrichment from sister cyst germ cells. Science, Vol. 352(6281), 95-99

Lindeman, R. E., \& Pelegri, F. (2010, April). Vertebrate maternal-effect genes: Insights into fertilization, early cleavage divisions, and germ cell determinant localization from studies in the Zebrafish. Molecular Reproduction and Development, Vol. 77, 299-313

Liu, K. C., Jacobs, D. T., Dunn, B. D., Fanning, A. S., \& Cheney, R. E. (2012). Myosin-X functions in polarized epithelial cells. Molecular Biology of the Cell, Vol. 23(9), 1675-1687

Loh, Y. H., Christoffels, A., Brenner, S., Hunziker, W., \& Venkatesh, B. (2004). Extensive expansion of the claudin gene family in the teleost fish, Fugu rubripes. Genome Research, Vol. 14(7), $1248-1257$

Markussen, F. H., Michon, A. M., Breitwieser, W., \& Ephrussi, A. (1995). Translational control of oskar generates short OSK, the isoform that induces pole plasm assembly. Development, Vol.121(11), 3723-3732

Marinos, E., \& Billett, F. S. (1981). Mitochondrial number, cytochrome oxidase and succinic dehydrogenase activity in Xenopus laevis oocytes. Journal of Embryology and Experimental Morphology, Vol.62, 395-409

Matter, K., \& Balda, M. S. (2003). Signalling to and from tight junctions. Nature Reviews Molecular Cell Biology, Vol.4(3), 225-236

Marlow, F. L., \& Mullins, M. C. (2008). Bucky ball functions in Balbiani body assembly and animalvegetal polarity in the oocyte and follicle cell layer in zebrafish. Developmental Biology, Vol. $321(1)$

McD WHITINGTON, B. P., \& Dixon, K. E. (1975). Quantitative studies of germ plasm and germ cells during early embryogenesis of Xenopus laevis. In Embryol. exp. Morph, Vol. 33

McWilliam, H., Li, W., Uludag, M., Squizzato, S., Park, Y. M., Buso, N., ... Lopez, R. (2013). Analysis Tool Web Services from the EMBL-EBI. Nucleic Acids Research, Vol. 41, 597-600

Miranda-Rodríguez, J. R., Salas-Vidal, E., Lomelí, H., Zurita, M., \& Schnabel, D. (2017). RhoA/ROCK pathway activity is essential for the correct localization of the germ plasm mRNAs in zebrafish embryos. Developmental Biology, Vol. 421, 27-42

Morais-de-Sá, E., Vega-Rioja, A., Trovisco, V., \& StJohnston, D. (2013). Oskar is targeted for degradation by the sequential action of Par-1, GSK-3, and the SCF-Slimbubiquitin ligase. Developmental Cell, Vol. 26, 303-314

Mullis, K., Faloona, F., Scharf, S., Saiki, R., Horn, G. and Erlich, H., 1986. Specific enzymatic amplification of DNA in vitro: the polymerase chain reaction. Cold Spring Harb Symp Quant 
Biol. 51 Pt 1, 263-73.

Nair, S., Marlow, F., Abrams, E., Kapp, L., Mullins, M. C., \& Pelegri, F. (2013). The Chromosomal Passenger Protein Birc5b Organizes Microfilaments and Germ Plasm in the Zebrafish Embryo. PLoS Genetics, Vol. 9(4)

Nakamura, A., Sato, K., \& Hanyu-Nakamura, K. (2004). Drosophila Cup is an eIF4E binding protein that associates with Bruno and regulates oskar mRNA Translation in Oogenesis. Developmental Cell, Vol. 6(1), 69-78

Neuhoff, V., Arold, N., Taube, D., Ehrhardt, W. (1988). Improved staining of proteins in polyacrylamide gels including isoelectric focusing gels with clear background at nanogram sensitivity using Coomassie Brilliant Blue. Electrophoresis, Vol.9, 255-262

Ng, B. F., Selvaraj, G. K., Mateos, C. S. C., Grosheva, I., Alvarez-Garcia, I., Martın-Bermudo, M. D., \& Palacios, I. M. (2016). A-Spectrin and Integrins Act Together To Regulate Actomyosin and Columnarization, and To Maintain a Monolayered Follicular Epithelium. Development (Cambridge), Vol.143(8), 1388-1399.

Nott, T. J., Petsalaki, E., Farber, P., Jervis, D., Fussner, E., Plochowietz, A., ... Baldwin, A. J. (2015). Phase Transition of a Disordered Nuage Protein Generates Environmentally Responsive Membraneless Organelles. Molecular Cell, Vol. 57, 936-947

Okada, H., Uezu, A., Mason, F. M., Soderblom, E. J., Moseley, M. A., \& Soderling, S. H. (2011). SH3 domain-based phototrapping in living cells reveals rho family GAP signaling complexes. Science Signaling, Vol. 4(201)

Palmer, J. F., \& Slack, C. (1970). Some bio-electric parameters of early Xenopus embryos. Journal of Embryology and Experimental Morphology, Vol. 24(3), 535-553

Pelegri, F., Knaut, H., Maischein, H. M., Schulte-Merker, S., \& Nüsslein-Volhard, C. (1999). A mutation in the zebrafish maternal-effect gene nebel affects furrow formation and vasa RNA localization. Current Biology. Vol. 9, 1431-1440

Pelegri, F., (2003). Maternal factors in zebrafish development. Developmental Dynamics. Vol. 228, 535-54.

Peng, K., Radivojac, P., Vucetic, S., Dunker, A. K., \& Obradovic, Z. (2006). Length-dependent prediction of protein in intrinsic disorder. BMC Bioinformatics, Vol. 7(208)

Pepling, M. E., Wilhelm, J. E., O’Hara, A. L., Gephardt, G. W., \& Spradling, A. C. (2007). Mouse oocytes within germ cell cysts and primordial follicles contain a Balbiani body. Proceedings of the National Academy of Sciences, Vol. vol. 104(1), 187-192

Polosello, C., Delon, I., Valenti, P., Ferrer, P., \& Payre, F. (2002). Dmoesin controls actin-based cell shape and polarity during Drosophila melanogaster oogenesis. Nature Cell Biology, Vol. 4(10), $782-789$ /ncb856

Raz, E. (2003). Primordial germ-cell development: The zebrafish perspective. Nature Reviews Genetics, Vol. 4, 690-700 
Regen, C. M., \& Steinhardt, R. A. (1986). Global properties of the Xenopus blastula are mediated by a high-resistance epithelial seal. Developmental Biology, Vol. 113(1), 147-154

Richter, K. N., Revelo, N. H., Seitz, K. J., Helm, M. S., Sarkar, D., Saleeb, R. S., ... Rizzoli, S. O. (2018). Glyoxal as an alternative fixative to formaldehyde in immunostaining and superresolution microscopy. The EMBO Journal, Vol. 37(1), 139-159

Riemer, S. (2014). Doctoral dissertation. Analyzing the molecular mechanism of Bucky ball localization during germ cell specification in zebrafish.

Riemer, S., Bontems, F., Krishnakumar, P., Gömann, J., \& Dosch, R. (2015). A functional Bucky ball-GFP transgene visualizes germ plasm in living zebrafish. Gene Expression Patterns, Vol. $18(1-2), 44-52$

Rongo, C., Gavis, E. R., \& Lehmann, R. (1995). Localization of oskar RNA regulates oskar translation and requires Oskar protein. Development, 121(9), 2737-2746

Roovers, E. F., Kaaij, L. J. T., Redl, S., Bronkhorst, A. W., Wiebrands, K., de Jesus Domingues, A. M., ... Ketting, R. F. (2018). Tdrd6a Regulates the Aggregation of Buc into Functional Subcellular Compartments that Drive Germ Cell Specification. Developmental Cell, Vol. 46(3), 285-301

Rupp, R. A. W., Snider, L., \& Weintraub, H. (1994). Xenopus embryos regulate the nuclear localization of XMyoD. Genes and Development, Vol. 8(11), 1311-1323.

Santos, A. C., \& Lehmann, R. (2004). Germ cell specification and migration in Drosophila and beyond. Current Biology, Vol. 14, 578-589.

Schindelin, J., Arganda-Carreras, I., Frise, E., Kaynig, V., Longair, M., Pietzsch, T., ... Cardona, A. (2012, July). Fiji: An open-source platform for biological-image analysis. Nature Methods, Vol. 9, 676-682.

Škugor, A., Tveiten, H., Johnsen, H., \& Andersen, Ø. (2016). Multiplicity of Buc copies in Atlantic salmon contrasts with loss of the germ cell determinant in primates, rodents and axolotl. BMC Evolutionary Biology, Vol. 16(232)

Smith, J. L., Wilson, J. E., \& Macdonald, P. M. (1992). Overexpression of oskar directs ectopic activation of nanos and presumptive pole cell formation in Drosophila embryos. Cell, Vol. 70(5), 849-859

Strasser, M. J., Mackenzie, N. C., Dumstrei, K., Nakkrasae, L. I., Stebler, J., \& Raz, E. (2008). Control over the morphology and segregation of Zebrafish germ cell granules during embryonic development. BMC Developmental Biology, Vol. 8(58)

Strassheim, D., May, L. G., Varker, K. A., Puhl, H. L., Phelps, S. H., Porter, R. A., ... Williams, C. L. (1999). M3 muscarinic acetylcholine receptors regulate cytoplasmic myosin by a process involving RhoA and requiring conventional protein kinase C isoforms. Journal of Biological Chemistry, Vol. 274(26), 18675-18685.

Strome, S., \& Updike, D. (2015, June 23). Specifying and protecting germ cell fate. Nature Reviews Molecular Cell Biology, Vol. 16, 406-416. 
Strome, S., \& Wood, W. B. (1983). Generation of asymmetry and segregation of germ-line granules in early C. elegans embryos. Cell, Vol. 35, 15-25

Stuart, R. O., \& Nigam, S. K. (1995). Regulated assembly of tight junctions by protein kinase C. Proceedings of the National Academy of Sciences, Vol. 92, 6072-6076

Tan, C. H., Lee, T. C., Weeraratne, S. D., Korzh, V., Lim, T. M., \& Gong, Z. (2002). Ziwi, the zebrafish homologue of the Drosophila piwi: Co-localization with vasa at the embryonic genital ridge and gonad-specific expression in the adults. Gene Expression Patterns, Vol. 2(3-4), 257260

Tang, L. (2019, January 1). Artificial biomolecular condensates. Nature Methods, Vol. 16, 21-27

Tay, H. G., Ng, Y. W., \& Manser, E. (2010). A vertebrate-specific Chp-PAK-PIX pathway maintains E-cadherin at adherens junctions during zebrafish epiboly. PLOS ONE, Vol. 5(4).

Theusch, E. V., Brown, K. J., \& Pelegri, F. (2006). Separate pathways of RNA recruitment lead to the compartmentalization of the zebrafish germ plasm. Developmental Biology, Vol. 292, 129141

Tompa, P. (2012, December). Intrinsically disordered proteins: A 10-year recap. Trends in Biochemical Sciences, Vol. 37, 509-516

Toretsky, J. A., \& Wright, P. E. (2014). Assemblages: Functional units formed by cellular phase separation. Journal of Cell Biology, Vol. 206, 579-588

Tsukita, S., \& Furuse, M. (2000, April 3). Pores in the wall: Claudins constitute tight junction strands containing aqueous pores. Journal of Cell Biology, Vol. 149, 13-16

Updike, D. L., Hachey, S. J., Kreher, J., \& Strome, S. (2011). P granules extend the nuclear pore complex environment in the C. elegans germ line. Journal of Cell Biology, Vol. 192(6), 939948

Urven, L. E., Yabe, T., \& Pelegri, F. (2006). A role for non-muscle myosin II function in furrow maturation in the early zebrafish embryo. Journal of Cell Science, Vol. 119, 4342-4352

van der Waal, M. S., Hengeveld, R. C. C., van der Horst, A., \& Lens, S. M. A. (2012). Cell division control by the Chromosomal Passenger Complex. Experimental Cell Research, Vol. 318, 407142

Vicente-Manzanares, M., Ma, X., Adelstein, R. S., \& Horwitz, A. R. (2009). Non-muscle myosin II takes centre stage in cell adhesion and migration. Nature Reviews Molecular Cell Biology, Vol. $10,778-789$

Villefranc, J. A., Amigo, J., \& Lawson, N. D. (2007). Gateway compatible vectors for analysis of gene function in the zebrafish. Developmental Dynamics, Vol. 236(11), 3077-3087.

Vitzthum, C., Stein, L., Brunner, N., Knittel, R., Fallier-Becker, P., \& Amasheh, S. (2019). Xenopus oocytes as a heterologous expression system for analysis of tight junction proteins. FASEB Journal : Official Publication of the Federation of American Societies for Experimental Biology, Vol. 33(4), 5312-5319 
von Wittich, W.H., 1845. Dissertatio Sistens Observationes Quaedam De Aranearum Ex Ovo Evolutione. Halis Saxonum, Halle, Germany.

Voronina, E., Seydoux, G., Sassone-Corsi, P., \& Nagamori, I. (2011). RNA granules in germ cells. Cold Spring Harbor Perspectives in Biology.

Wang, K., Wloka, C., \& Bi, E. (2019). Non-muscle Myosin-II Is Required for the Generation of a Constriction Site for Subsequent Abscission. IScience, Vol. 13, 69-81

Watanabe, T., Sato, K., \& Kaibuchi, K. (2009). Cadherin-mediated Intercellular Adhesion and Signaling Cascades Involving Small GTPases. Cold Spring Harbor Perspectives in Biology.

Westerfield, M., 2000. The zebrafish book. A guide for the laboratory use of zebrafish (Danio rerio). 4th ed., Univ. of Oregon Press, Eugene.

Willott, E., Balda, M. S., Fanning, A. S., Jameson, B., Van Itallie, C., \& Anderson, J. M. (1993). The tight junction protein ZO-1 is homologous to the Drosophila discs-large tumor suppressor protein of septate junctions. Proceedings of the National Academy of Sciences of the United States of America, Vol. 90(16), 7834-7838

Wylie, C. C., \& Heasman, J. (1976). The formation of the gonadal ridge in Xenopus laevis I. A light and transmission electron microscope study. In Embryol. exp. Morph, Vol. 35

Yabe, T., Ge, X., Lindeman, R., Nair, S., Runke, G., Mullins, M. C., \& Pelegri, F. (2009). The maternal-effect gene cellular island encodes Aurora B kinase and is essential for furrow formation in the early zebrafish embryo. PLoS Genetics, Vol. 5(6)

Yabe, T., Ge, X., \& Pelegri, F. (2007). The zebrafish maternal-effect gene cellular atoll encodes the centriolar component sas-6 and defects in its paternal function promote whole genome duplication. Developmental Biology. Vol. 312(1), 44-60

Yoon, C., Kawakami, K., \& Hopkins, N. (1997). Zebrafish vasa homologue RNA is localized to the cleavage planes of 2- and 4-cell-stage embryos and is expressed in the primordial germ cells. Development, 124(16), 3157-3165.

Yu, D., Marchiando, A. M., Weber, C. R., Raleigh, D. R., Wang, Y., Shen, L., \& Turner, J. R. (2010). MLCK-dependent exchange and actin binding region-dependent anchoring of ZO-1 regulate tight junction barrier function. Proceedings of the National Academy of Sciences of the United States of America, Vol. 107(18), 8237-8241

Zhang, G., Wang, Z., Du, Z., \& Zhang, H. (2018). mTOR Regulates Phase Separation of PGL Granules to Modulate Their Autophagic Degradation. Cell, Vol. 174(6), 1492-1506

Zheng, J., Gao, M., Huynh, N., Tindell, S. J., Vo, H. D. L., McDonald, W. H., \& Arkov, A. L. (2016). In vivo mapping of a dynamic ribonucleoprotein granule interactome in early Drosophila embryos. FEBS Open Bio, Vol. 6, 1248-1256

Zhuo, S., Clemens, J. C., Hakes, D. J., Barford, D., \& Dixon, J. E. (1993). Expression, purification, crystallization, and biochemical characterization of a recombinant protein phosphatase. Journal of Biological Chemistry, Vol. 268(24), 17754-17761 


\section{Curriculum Vitae}

\section{Alexander Goloborodko}

Date of birth: March 12, 1990

Place of birth: Poltava, Ukraine

Nationality: Dutch

Private address: Rhönring 54, 64289, Darmstadt

Mobile: +4915253678411

E-mail:Alexander.A.Goloborodko@gmail.com

\section{Research experience}

04/16 - 10/19 Graduate research

Center for Molecular Biosciences, Department of Developmental Biochemistry, Göttingen, Germany Supervisor: Dr. Roland Dosch

- Investigated fundamental principles of sexual reproduction, by using Bucky ball protein as a molecular proxy to study germ plasm localization

- Optimized molecular biology and biochemical methods for work in a model organism zebrafish

- Developed research projects for bachelor and master students

- Supervised and mentored bachelor, master and PhD students

- Actively participated in institutional and lab seminars, national and international research meetings

11/14 - 08/15 Undergraduate research

University Medical Center, Laboratory of Genetics and functional genomics of Usher syndrome, Nijmegen,

Netherlands

Supervisor: Prof. Hannie Kremer

- Developed a novel transgenic system to test a therapy for Usher syndrome and understand underlying neurodegenerative mechanisms.

- Developed, optimized and successfully applied a novel genome editing method based on homologous recombination using the CRISPR/Cas9 system.

- Successfully produced antisense oligonucleotides aimed to target genetic defects in Usher syndrome patients.

11/13 - 07/14 Undergraduate research

Radboud Institute of Molecular Life Sciences, Laboratory of Tumor Immunology, Nijmegen, Netherlands Supervisor: Dr. Jonas Søndergaard

- Investigated the role of cell cycle inhibitor $\mathrm{p} 15$, the cytoskine TGF- $\beta$ and the transcriptional modulator DC-SCRIPT in the MCF-7 breast cancer cells 


\section{Education}

4/2016 - 10/2019 PhD in Developmental Biochemistry at Georg August University of Göttingen, Germany 9/2013 - 2/2015 Master of Molecular Life Sciences at Radboud University Nijmegen, Netherlands 9/2009 - 8/2013 Bachelor of Molecular Life Sciences at Radboud University, Netherlands, B.Sc. 9/2006 - 8/2009 Secondary school Petrus Canisius College, Alkmaar, Netherlands

\section{Scientific and Methodological expertise}

- Animal model (zebrafish): protein complex immunoprecipitation, protein analysis by SDS-PAGE, microinjection, immunohistochemistry, live imaging, genotyping.

- CRISPR/Cas9 system: from gRNA design to the detection of genomic modifications

- Molecular cloning techniques: In Fusion, Gateway, T4 Ligase, TOPO cloning

- Molecular techniques: Western blot, Fluorescent cell sorting, (q)PCR

- Tissue culture: HEK293 and MCF7 cells

- Microscopy: confocal laser scanning microscopy and fluorescent digital stereoscopy

\section{IT skills}

- Basic programming in MATLAB

- Processing and analyzing images in ZEN, ImageJ and Adobe Photoshop

- Composing figures with Adobe Illustrator

- Analyzing sequencing results with Seqman and Snapgene

- Analyzing genes in BLAST and Ensemble genome browser

\section{Teaching}

- Developed lab rotation and master thesis projects on annual basis

- Supervised ten laboratory rotations (2 month each) and one master thesis (6 month)

- Supervised Biochemistry practical course for medical students (6 weeks in total)

- Lead a tutorial for Molecular Biology students (2 hours in total)

- Supervised newcomer PhD students (2 students)

\section{Grants}

- Travel grant for the Meeting of German and Japanese Society of Developmental Biologists, Kiel (15-18 March 2017), provided by the Göttingen Graduate School for Neurosciences, Biophysics, and Molecular Biosciences

- $\mathrm{PhD}$ grant for duration of 6 months (14.04 - 14.10.2019) from Göttingen Graduate Center for Neurosciences, Biophysics, and Molecular Biosciences

\section{Conferences and workshops}

- 4/18 Oral presentation "Molecular mechanisms of germ plasm localization during early zebrafish development", Regional Fishmeeting, Heidelberg, Germany

- 5/17 Workshop "Genome Engineering in Zebrafish", Frankfurt, Germany 
- 3/17 Poster presentation "Molecular mechanisms of germ plasm localization during early zebrafish development", GfE Meeting of the German and Japanese Society of Developmental Biologists, Kiel, Germany

- 9/16 Poster presentation "Molecular mechanisms of germ plasm localization during early zebrafish development", $11^{\text {th }}$ GfE School: Cell Dynamics in Development \& Evolution, Günzburg, Germany

\section{Languages}

- English (fluent), Dutch (fluent), German (moderate), Russian (fluent), Ukrainian (fluent)

\section{Publications}

Krishnakumar P., Riemer S., Perera R., Lingner T., Goloborodko A., Khalifa H., Bontems F., Kaufholz F., El-Brolosy M.A., Dosch R. Functional equivalence of germ plasm organizers. (2018) PLoS Genet.

Slijkerman R.W.N., Goloborodko A., Hetterschijt L., Peters T.A., Gerits M., Kremer H., Van Wijk

E. Poor splice site recognition in a humanized zebrafish knock-in model for the recurrent deep-intronic c.7595-2144A $>$ G mutation in $U S H 2 A$. (2018) Zebrafish.

\section{References}

\section{Dr. Roland Dosch}

Dept. of Developmental Biochemistry Georg-August-University Göttingen, Germany E-mail: roland.dosch@med.uni-goettingen.de Tel. +49 (0)551-39 5970

\section{Dr. Jonas Søndergaard}

Dept. of Microbiology Tumor and Cell Biology Karolinska University, Solna, Sweden

E-mail: Jonas.Sondergaard@ ki.se

Tel. +46762834623 